Ivan Silvestre Paganini Marin

\title{
Aperfeiçoamento do Método de Elementos Analíticos para Simulação de Escoamento em Rochas Porosas Fraturadas
}





\title{
Aperfeiçoamento do Método de Elementos Analíticos para Simulação de Escoamento em Rochas Porosas Fraturadas
}

\author{
Tese apresentada à Escola de Engenharia de \\ São Carlos como parte dos requisitos para a \\ obtenção do Título de Doutor em Ciências. \\ Área de Concentração: Engenharia Hi- \\ dráulica e Saneamento
}

Orientador:

Prof. Tit. Edson Wendland

Versão Corrigida

São Carlos - SP

Outubro / 2011 
AUTORIZO A REPRODUČ̃O E DIVULGAÇÃO TOTAL OU PARCIAL DESTE TRABALHO, POR QUALQUER MEIO CONVENCIONAL OU ELETRÔNICO, PARA FINS DE ESTUDO E PESQUISA, DESDE QUE CITADA A FONTE.

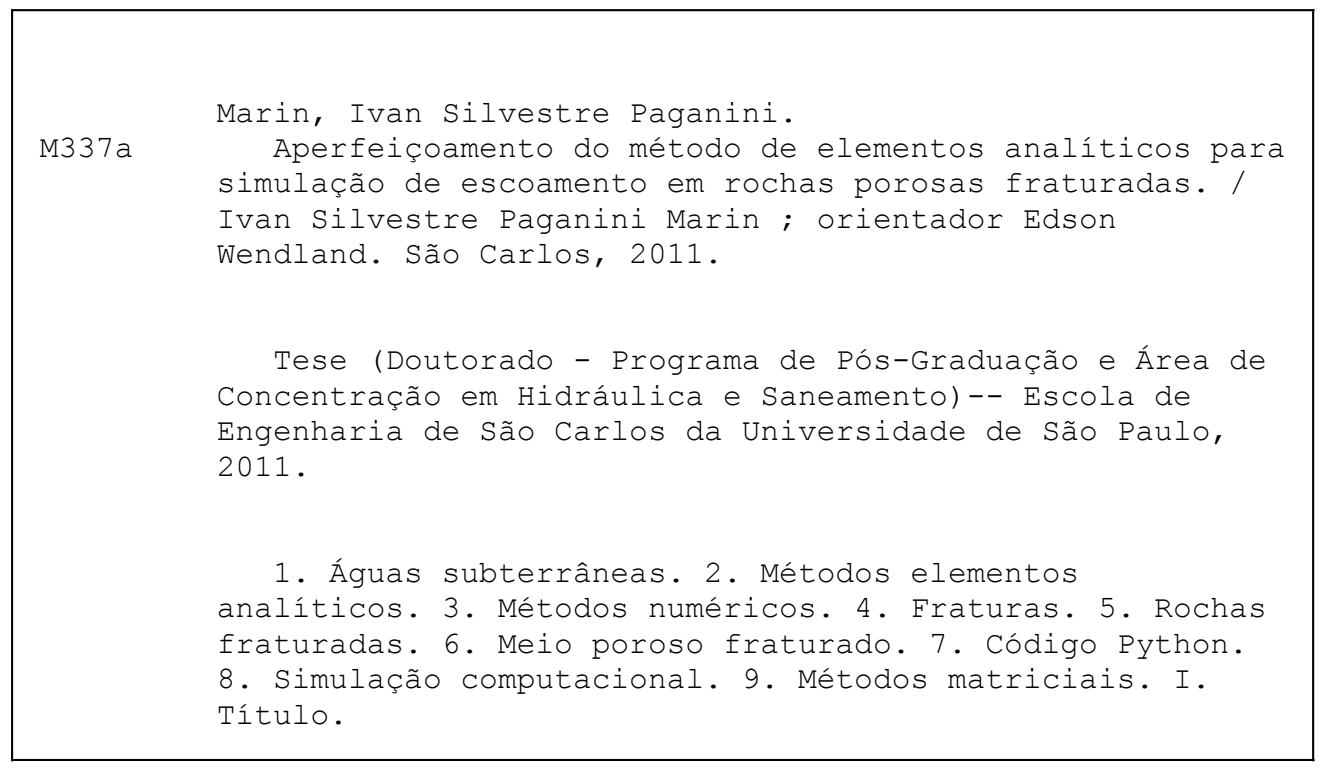




\section{FOLHA DE JULGAMENTO}

Candidato: Bacharel IVAN SILVESTRE PAGANINI MARIN

Título da tese: "Aperfeiçoamento do método de elementos analíticos para simulação de escoamento em rochas porosas fraturadas".

Data da defesa: 07/10/2011:

\section{Comissão Julgadora:}

Prof. Titular Edson Cezar Wendland (Orientador)

(Escola de Engenharia de São Carlos/EESC)

Prof. Titular José Alberto Cuminato

(Instituto de Ciências Matemáticas e Computação/ICMC)

Prof. Associado José Eduardo Martinho Hornos

(Instituto de Física de São Carlos/IFSC)

Prof. Associado Eurípedes do Amaral Junior

(Pontifícia Universidade Católica do Rio de Janeiro / PUC-Rio)

Prof. Titular Jaime Joaquim da Silva Pereira Cabral (Universidade Federal de Pernambuco/UFPE)
Resultado:
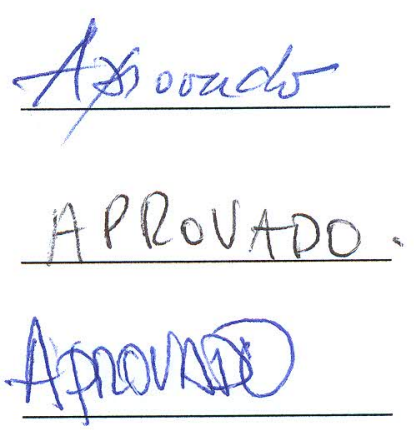
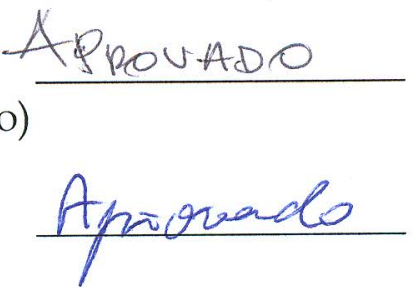

Coordenador do Programa de Pós-Graduação em Engenharia Hidráulica e Saneamento:

Prof. Titular Edson Cezar Wendland

Presidente da Comissão de Pós-Graduação:

Prof. Associado Paulo Cesar Lima Segantine 



\section{Resumo}

\section{MARIN, I S P. Aperfeiçoamento do Método de Elementos Analíticos para Simulação de}

Escoamento em Rochas Porosas Fraturadas. 2011. 152p. Tese (Doutorado) - Escola de Engenharia de São Carlos, Universidade de São Paulo, 2011.

Escoamento de água subterrânea em meios porosos fraturados é um problema de grande importância, principalmente nos contextos de petróleo, energia geotérmica e repositórios geológicos. Com o aquecimento da Terra, a geração de energia com baixa emissão de gases estufa torna-se imperativa, considerando o crescimento de uso de energia e o impacto do aquecimento global. Dentre as opções disponíveis para geração de energia, a energia nuclear apresenta-se como candidata. Entretanto, dentre os riscos do uso de energia nuclear, o destino do combustível usado e de materiais provenientes de descomissionamento é um problema em aberto. Repositórios geológicos surgem como uma alternativa para a estocagem de médio e longo prazo, por serem capazes de proporcionar isolamento em escalas geológicas de tempo. O principal vetor de propagação do material radioativo estocados em repositórios é a água subterrânea, e meios fraturados estão presentes na maioria dos domínios. Fraturas podem propagar a água subterrânea e, portanto, solutos com velocidades muito maiores que as do meio poroso. Além disso, fraturas são, geralmente, sistemas multiescala, em que diferentes escalas - de centímetros a kilômetros - podem ter um papel significativo. Métodos como elementos finitos, apesar de representarem certos comportamentos do escoamento em fraturas, têm dificuldade em simular sistemas com grandes diferenças de escala, já que necessitam de discretização do domínio. O Método de Elementos Analíticos (MEA) surge como uma alternativa a esse problema, pois não necessita de discretização de domínio, podendo simular características hidrogeológicas em diferentes escalas. Este trabalho tem como proposta aperfeiçoar o MEA, desenvolvendo um elemento analítico para fraturas que interagem com o meio poroso, aplicando os desenvolvimentos recentes na teoria do Método. Baseado na Integral de Cauchy e em transformações de coordenadas, o novo formalismo de solução no plano $\chi$ permite maior precisão na imposição das condições de contorno, sendo aplicado para inomogeneidades circulares, inomogeneidades poligonais formadas por line doublets e para o elemento de fratura. Dificuldades numéricas na simulação para fraturas levaram ao desenvolvimento de um método matricial de solução, aplicado com sucesso para todos os elementos apresentados neste trabalho. Soluções exatas para a inomogeneidade circular e para uma fratura foram comparadas com inomogeneidades poligonais equivalentes, com sucesso. O método matricial permitiu também um estudo da convergência do método iterativo e possibilita a melhoria do Método de Elementos Analíticos em geral.

Palavras-chave: Escoamento de água subterrânea. Fraturas. Método de Elementos Analíticos. Simulação. 


\section{Abstract}

MARIN, I S P. Improving the Analytic Element Method for Simulation of Flow in Fractured Porous Rock. 2011. 152p. Tese (Doutorado) - Escola de Engenharia de São Carlos, Universidade de São Paulo, 2011.

Groundwater flow in fractured porous media is a recent and modern problem, considering the petroil, geothermic energy and geologic repositories context. As the Earth warms, low CO2 energy generation is paramount, when the projections of energy demand and worsening of the global warming effects are factored in. Nuclear energy generation appears as one of the canditates to generate electricity with low $\mathrm{CO} 2$ emissions. Several factors must be considered, thought, when nuclear energy is concerned. The spent nuclear fueld and the decomission residues must be safely stored for long periods of time. One of the alternatives for mid and long term disposal is the use of geological repositories. Because of its characteristics, groundwater studies must be conducted to assert the safety of the repositories, as it's the main contaminant vector for the stored nuclear material. Fractures must be considered in those studies, as they are usually present in almost all settings considered for repositories, and can propagate groundwater (and dissolved solutes) with very high speeds, several orders of magnitude faster than the porous media. Fractures also forms multiscale problems, where different problem scales - from centimeters to kilometers - can influence the behavior of the groundwater flow and the consequent solute transport. The usual groundwater simulation methods, even when capable of including fracture phenomena, have problems with the scale differences, as they usually depend on domain discretization. The Analytic Element Method is based on analytic solutions of the groundwater governing equations and does not depend on domain discretization, being able to tackle multiscale problems that the other methods can't produce a feasible solution. The Analytic Element Method has been developed in recent years and has been applied in different fields, as wellhead protection area delineation. This work proposes to improve the Analytic Elemento Method developing an analytic element for flow in fractures, using the recent developments as the direct use of Cauchy Integrals in the $\chi$ plane. These new developments allow increased precision on the numerical boundary conditions matching. This method is applied on circular inhomogeneities, polygonal inhomogeneities modelled by line doublets and the fracture element. Numerical problems in the boundary condition matching for the fractures led to the development of matrix solution method, used on all elements presented in this work. Exact solutions for one circular inhomogeneity and for one fracture allowed comparison with the numerical ones, with satisfactory results. The matrix method also permitted a convergence study of the iterative methods, possibilitating for the general improvement of the Analytic Element Method.

Keywords: Groundwater Flow. Fractures. Analytic Element Method. Numerical Simulation. 


\section{Agradecimentos}

Agradeço ...

Comecei a escrever esses agradecimentos na minha cabeça várias vezes. E cada vez que eu começava, lembrava da história deste doutorado. Dois países, dois orientadores, muitas viagens, momentos de desespero e alegria. Amigos que vieram, amigos que foram, amigos que ficaram, do mundo inteiro. Como agradecer a todo mundo que ajudou, de uma forma ou de outra, por todos esses anos? Seria injusto, entretanto, não tentar.

Começo então pela família. Afinal, nada mais justo, já que comecei deles. Pai, Mãe e Irmã, Gil, Léa e Marcela, os únicos que, muitas vezes sem entender o que eu estava fazendo com o tal do doutorado, continuavam dando apoio, suporte, comida de domingo e carinho. E o pior, tudo isso e muito mais de forma incondicional, mesmo com o mau humor, a demora para ligar de volta, os resmungos. Ser pai, mãe e irmã não deve ser fácil. E sem vocês fica impossível.

Penso então em meus amigos. Como colocar em uma lista? Pior, em uma lista ordenada? Como agradecer pelo o que cada um fez por mim? Tarefa impossível. Desisto, coloco sem ordem mesmo: Gerson, Matheus (Rato), Lucas Magali, Daniel Silvestre, Weslley Tim, Marina Magaton, Marcel, Ivanhoé e Camila, Isabella, Bruno Nagumo. Pessoal de Åsgard, Gerson, Matheus (Rato), Lucas, Rafael SPA, Hugoooooo, Léo, Magnus, Lê, Rodrigo, Thiago. Pessoal da 8, Chaves, que me acolheu em sua casa no momento difícil, e Deodato. Folks from the "International Shelter for Crazy People", Brett, Matt, Sharad, Cam, Moe, Dave, Sid, Herbert Júnior, Ashley. Friends from Minneapolis, André, Eddie, Jamie, Marcela, Alya, Andrew, the russians, the folks from Al's, Justin, Allison, Jill. My inseparable friend, Bonnie, my very close friend, Becky, and Emily, who believed in me when no one else would. Cada um de vocês, every single one of you, me ajudaram, me carregaram, me deram bronca, e me salvaram de infinitas enrascadas e sufocos. Sou eternamente grato, I'm forever grateful.

E existe também o dia a dia, a labuta diária, o pequeno andar que muitas vezes não nos apercebemos mas que é fundamental na construção de um trabalho. Meus agradecimentos para todos do LHC, Mariano e José Anderson (a equipe AEM Brasil), Maldonado, Marcuzzo (Frango), Fernanda, Patrick, Camargo!, Taiana, Gomes, Bergamo, aqueles que me acolheram no LHC, aos mais novos, Chaves (kombi), Cabrera, Manzione, Murilo, Aline, Marjolly, Cristian, Tati, e até aos recém-chegados, Antônio Frota, Davi, Paulo Tarso, e aos ICs, Rafael, Bim e Wicher. Foi com vocês, todos vocês, que eu passei todos esses anos, todo dia, compartilhando os momentos de descontração, as pequenas e grandes derrotas e vitórias que o trabalho em equipe nos permitiu.

My profound gratitute to Prof. Otto Strack and his wife, Andrine, for accepting me as a visiting scholar, for all the insights and teachings, the many things (both professional and personal) that I've learned with you on my time there, and helping me with all the hurdles that my travel to the USA had. I'm also profoundly grateful for receiving me in your house, and with the patience that Prof. 
Strack and Andrine listened to my questions and gave the answers that I needed, not the ones that I wanted. My sincere Thank You.

Obrigado a todos os funcionários do SHS, Sá, Pavi, Rose, André, Flávia e Fernanda, e todos os demais, pelo suporte e pela paciência. Also many thanks for the integrants of the "Groundwater Group", especially Philippe Le Grand and Prof. Randall Barnes, for the wise advices and the most helpful discussions and insights.

Agradeço também ao Prof. José Eduardo M. Hornos, do IFSC, que além de ter me proporcionado a porta de entrada para este projeto, mostrou diferentes caminhos para avanços importantes na Ciência para o Brasil e para o mundo. O Prof. Hornos também me manteve com um pé na realidade e um pé no futuro, me questionando, muitas vezes de forma provocadora, o que eu achava que sabia.

Ao meu orientador, Edson Wendland, agradeço profundamente por ter acreditado em um físico perdido. O Prof. Edson me ensinou a apreciar o planeta em que vivemos, especialmente o subterrâneo, e o abraçar com as técnicas numéricas. Mas, mais importante, aprendi com ele a ter uma vida profissional como cientista, mesmo na engenharia; como ver um problema e procurar não a melhor solução, mas a melhor solução possível; e ao mesmo tempo manter uma amizade, me ajudando muito além das responsabilidades de um orientador. 

"It was the best of times, it was the worst of times..." Charles Dickens

"Algumas pessoas dizem, "Como você consegue viver sem saber?"Eu não compreendo o que eles querem dizer. Eu vivo sempre sem saber. Isto é fácil. Como você faz para saber é o que eu quero saber." Richard Feynman

"It's not the years, honey, it's the mileage. "Indiana Jones 


\section{Sumário}

\section{Lista de Figuras}

Lista de Símbolos

Lista de Abreviações

1 Introdução $\quad 23$

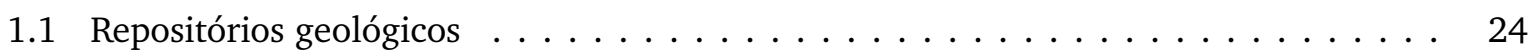

1.2 Escoamento em Fraturas . . . . . . . . . . . . . . . . . . . . . . 24

1.3 Modelagem Numérica $\ldots \ldots \ldots \ldots \ldots \ldots \ldots \ldots \ldots \ldots$

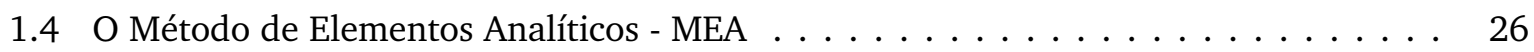

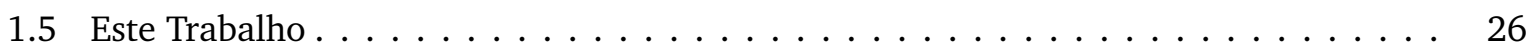

2 Hidrogeologia $\quad 29$

2.1 Introdução . . . . . . . . . . . . . . . . . . . . . . . . . . 29

2.2 Definições de Hidrogeologia $\ldots \ldots \ldots$. . . . . . . . . . . . . . . . 29

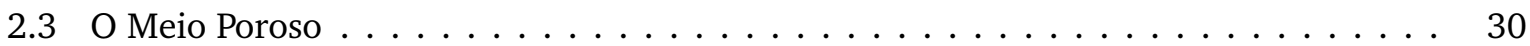

2.3.1 A Aproximação Contínua para Meios Porosos . . . . . . . . . . . . . . . . . 30

2.3.2 A Aproximação Contínua Para O Meio Fraturado . . . . . . . . . . . . . . . . . 31

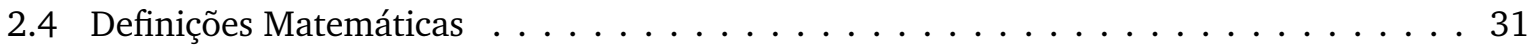

2.4.1 Carga Hidráulica . . . . . . . . . . . . . . . . . . . . . . . . 31

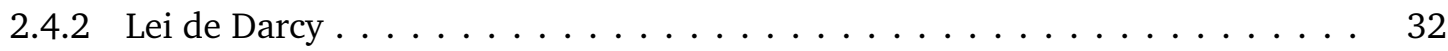

2.4.3 Vetor Descarga e o Potencial de Descarga . . . . . . . . . . . . . . . 33

2.4.4 Equação da Continuidade e a Equação de Laplace . . . . . . . . . . . . . . . . 35 
3.1 Características de Fraturas $\ldots \ldots \ldots \ldots \ldots \ldots \ldots \ldots \ldots$

3.1.1 Características Físicas e Geométricas . . . . . . . . . . . . . . . . . . . 41

3.1 .2 Interconexão entre Fraturas $\ldots \ldots \ldots \ldots \ldots \ldots$. . . . . . . . . . 43

3.1.3 Limites na Descrição de Sistemas de Fraturas . . . . . . . . . . . . . . . . 44

3.2 Escoamento em Uma Fratura . . . . . . . . . . . . . . . . . . . . . . . . . 44

3.3 Escoamento em uma Rede de Fraturas . . . . . . . . . . . . . . . . . . . . . . 46

3.3.1 Modelos Contínuos para um Sistema de Fraturas . . . . . . . . . . . . . . . . 47

3.3.2 Modelos Discretos para um Sistema de Fraturas . . . . . . . . . . . . . . . . 47

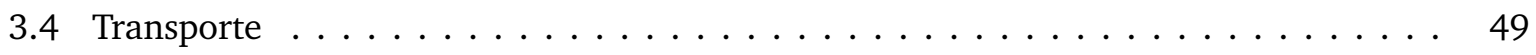

3.5 Modelos e Abordagens Numéricas para Sistemas Fraturados . . . . . . . . . . . . 51

3.5.1 Modelo de Placas Paralelas - a Lei Cúbica . . . . . . . . . . . . . . . . . . . . 51

3.5.2 Aproximação Linear para a Abertura . . . . . . . . . . . . . . . . 53

4 O Método de Elementos Analíticos

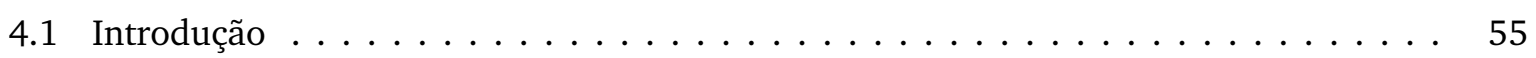

4.2 O Método de Elementos Analíticos $\ldots \ldots \ldots \ldots \ldots \ldots$

4.2 .1 Superposição . . . . . . . . . . . . . . . . . . . . . . 57

4.3 Satisfazendo as Condições de Contorno $\ldots \ldots \ldots \ldots \ldots \ldots \ldots$

4.3 .1 Método de Colocação . . . . . . . . . . . . . . . . . . . . . . 59

4.3 .2 Método dos Mínimos Quadrados . . . . . . . . . . . . . . . . . . . 59

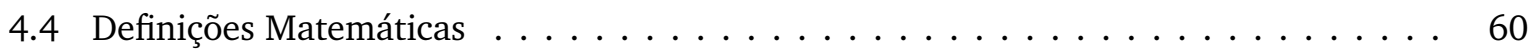

4.4 .1 Transformações de Coordenadas . . . . . . . . . . . . . . . . . . . . 60

4.4 .2 Funções Holomórficas, Analíticas e Harmônicas . . . . . . . . . . . . . . . . . 62

4.4.3 Teorema de Cauchy-Goursat e a Fórmula Integral de Cauchy . . . . . . . . . 63

4.4.4 Relação entre a Fórmula Integral de Cauchy e a Série de Taylor . . . . . . . . . 64

4.4 .5 Polinômios de Chebyshev . . . . . . . . . . . . . . . . . . . . . . . 64 


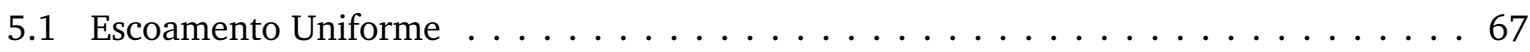

5.2 Inomogeneidades Circulares $\ldots \ldots \ldots \ldots \ldots \ldots \ldots$

5.2 .1 Condição de Contorno $\ldots \ldots \ldots \ldots \ldots \ldots \ldots$

5.2.2 Solução Exata para uma Inomogeneidade Circular e Escoamento Uniforme . . 69

5.3 Line Doublets e Inomogeneidades Poligonais . . . . . . . . . . . . . . . . . . . . 69

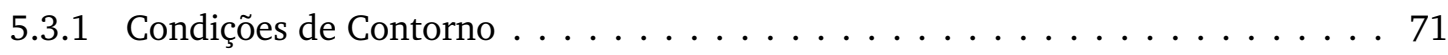

5.3 .2 Definição do Potencial . . . . . . . . . . . . . . . . . . . . 73

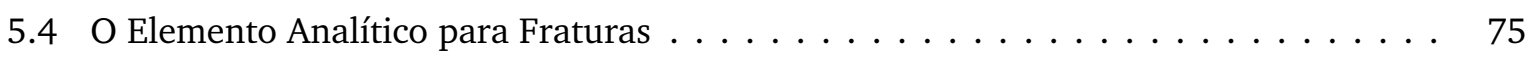

5.4 .1 Condições de Contorno . . . . . . . . . . . . . . . . . . . . . 75

5.4 .2 Solução Analítica para uma Fratura . . . . . . . . . . . . . . . . . . . . 79

5.4 .3 Descontinuidade no Potencial da Fratura . . . . . . . . . . . . . . . 80

5.4.4 Potencial Complexo para mais de uma Fratura $\ldots \ldots \ldots \ldots$. . . . . . . 81

6 Algoritmos de Solução e Implementação Numérica 83

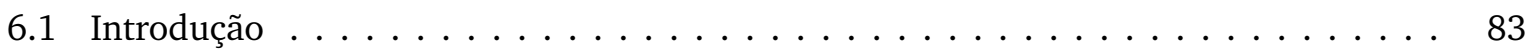

6.2 Condições de Contorno e a Integral de Cauchy $\ldots \ldots \ldots \ldots \ldots \ldots$. . . . . . . 84

6.2 .1 Forma Geral . . . . . . . . . . . . . . . . . . . . . 84

6.3 Inomogeneidades Circulares $\ldots \ldots \ldots \ldots \ldots \ldots \ldots \ldots \ldots$

6.3.1 Ortogonalidade da Série de Fourier $\ldots \ldots \ldots \ldots \ldots \ldots$

6.3 .2 Mínimos Quadrados . . . . . . . . . . . . . . . . . . . . . 86

6.4 Line Doublets e Inomogeneidades Poligonais . . . . . . . . . . . . . . . . . . 88

6.4 .1 Ortogonalidade da Série de Fourier $\ldots \ldots \ldots \ldots \ldots$

6.4 .2 Integral de Cauchy . . . . . . . . . . . . . . . . . . . . . . 89

6.4 .3 Mínimos Quadrados . . . . . . . . . . . . . . . . . . . . . 92

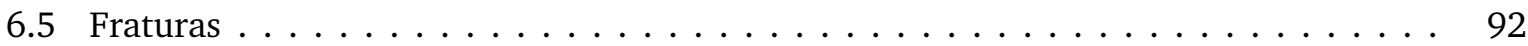

6.5 .1 Ortogonalidade via Série de Fourier $\ldots \ldots \ldots \ldots \ldots$

6.5 .2 Integral de Cauchy $\ldots \ldots \ldots \ldots \ldots \ldots \ldots \ldots \ldots$ 
6.6 Algoritmos de Solução . . . . . . . . . . . . . . . . . . . . . . . . . . 94

6.6.1 Algoritmo de Solução Iterativo Implícito ‥ . . . . . . . . . . . . . . 95

6.6 .2 Algoritmo de Solução Matricial . . . . . . . . . . . . . . . . . . . . . 97

6.6.3 Forma Matricial para Inomogeneidade Circular . . . . . . . . . . . . . 98

6.6.4 Forma Matricial para Inomogeneidade Poligonal _ . . . . . . . . . . . . . 99

6.6 .5 Forma Matricial para Fratura $\ldots \ldots \ldots \ldots \ldots$

6.6 .6 Forma Geral das Integrais $\ldots \ldots \ldots \ldots \ldots \ldots$

6.6.7 Algoritmo de Solução Iterativo Explícito e Partição da Matriz . . . . . . . . . 101

6.7 Integração Numérica versus Mínimos Quadrados . . . . . . . . . . . . . . . . 102

6.8 Linguagens de Programação e Implementação do Código . . . . . . . . . . . . . 103

6.9 Análise de Erro . . . . . . . . . . . . . . . . . . . . . . . . 104

6.9 .1 Convergência Numérica $\ldots \ldots \ldots \ldots$. . . . . . . . . . . 105

$\begin{array}{llr}7 & \text { Resultados } & 107\end{array}$

7.1 Inomogeneidades Circulares $\ldots \ldots \ldots \ldots$. . . . . . . . . . . . . 109

7.1.1 Comparação da Solução obtida usando o Algoritmo Iterativo Implícito e o Matricial com a Solução Exata . . . . . . . . . . . . . . . . . . . . . 109

7.1.2 Simulação para várias Inomogeneidades Circulares . . . . . . . . . . . . . . 111

7.2 Line Doublets para Inomogeneidades Poligonais f . . . . . . . . . . . . . . . . 112

7.2.1 Comparação da Solução Exata para uma Inomogeneidade Circular com a Aproximação Poligonal por line doublets . . . . . . . . . . . . . . . . . 112

7.2.2 Simulação para Duas Inomogeneidades Poligonais . . . . . . . . . . . . 117

7.2.3 Variação na Ordem da Expansão para a line doublet $\ldots \ldots$. . . . . . . . 118

7.2.4 Algoritmo Iterativo Explícito para a Inomogeneidade Poligonal formada por line doublets . . . . . . . . . . . . . . . . . . . . . . . 118

7.3 Fraturas . . . . . . . . . . . . . . . . . . . . . . . . . 119

7.3.1 Comparação da Solução Analítica para uma Fratura e uma Inomogeneidade Poligonal . . . . . . . . . . . . . . . . . . . . . . . . . . . . 119

7.3.2 Algoritmo Iterativo Explícito para o Elemento para Fraturas . . . . . . . . . 122 
7.3.3 Simulação para cruzamento de Fraturas ～. . . . . . . . . . . . . . . . . . . 122

7.3.4 Simulação de alguns sistemas fraturados . . . . . . . . . . . . . . . . . . . 124

8 Conclusões e Perspectivas Futuras $\quad 129$

8.1 Inomogeneidades Circulares . . . . . . . . . . . . . . . . . . . . . . . 129

8.2 Inomogeneidades Poligonais formadas por Line Doublets . . . . . . . . . . . . . . 130

8.3 Fraturas . . . . . . . . . . . . . . . . . . . . . . 130

8.4 O Método Matricial . . . . . . . . . . . . . . . . . . . . . . . . 131

8.5 Programação Orientada a Objetos e o MEA . . . . . . . . . . . . . . . . . . . . 132

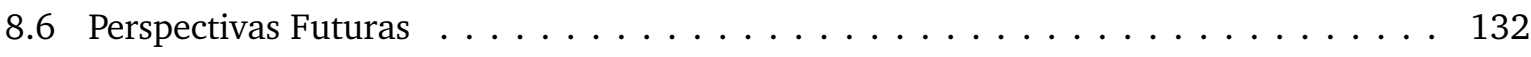

$\begin{array}{ll}\text { Referências Bibliográficas } & 135\end{array}$

Apêndice A - Complementos de Hidrologia 143

A.1 Equação de Bernoulli e Lei de Darcy . . . . . . . . . . . . . . . . . . . . . . . . 143

A.1.1 Lei de Darcy . . . . . . . . . . . . . . . . . . . . . . . . . . . 144

Apêndice B - A Aproximação de Dupuit-Forchheimer 145

Apêndice C - Matriz de Interação para uma Line Doublet 147

Apêndice D - Relações de Ortogonalidade 149

D.1 Relações de Ortogonalidade Contínuas . . . . . . . . . . . . . . . . . . . . . . . . 149

D.2 Relações de Ortogonalidade Discretas $\ldots \ldots \ldots \ldots \ldots \ldots \ldots$. . . . . . 150

Apêndice E - Desenvolvimentos Adicionais $\quad 151$

E.1 Simulador do Método de Elementos Analíticos - SMEAgol e o Visu . . . . . . . . . . . . 151 



\section{Lista de Figuras}

2.1 Esquema de um piezômetro em um aquífero livre. $\phi$ é a carga hidráulica, $\frac{p}{\gamma}$ é a carga de pressão e $z$, a carga de elevação. . . . . . . . . . . . . . . . . .

2.2 Escoamento unidimensional com transição do regime confinado para o regime não confinado. Adaptado de (1). . . . . . . . . . . . . . . . . . . . . . . . . .

2.3 Esquema do vetor de descarga específica para um bloco. Adaptado de (1). . . . . . .

3.1 Reproduzida de Klimczak et al. (2). Perfil de uma fratura em um afloramento rochoso. A figura $a$ é uma fotografia, enquanto a figura $b$ é um desenho feito por um geólogo evidenciando o sistema de fraturas. . . . . . . . . . . . . . . . . . .

3.2 Reproduzida de Crandall et al. (3). Simulação de escoamento usando elementos finitos em uma fratura com paredes rugosas. Diferença de pressão entre o lado esquerdo e direito de $\Delta P=100 P a$, Permeabilidade do meio poroso $\kappa=200 \mathrm{md}$. Comprimento da fratura: $10 \mathrm{~cm}$. Permeabilidade da fratura $\kappa=362 \mathrm{md}$, onde $m d$ denota $1 e^{-3}$ darcys. . 41

4.1 Fotografia Aérea da Bacia Piloto do Ribeirão da Onça. . . . . . . . . . . . . . . . . 57

4.2 Fotografia Aérea da Bacia Piloto do Ribeirão da Onça, com discretização dos elementos

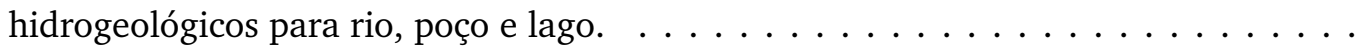

4.3 Inclusão de uma inomogeneidade na condutividade hidráulica por um polígono formado por line doublets. . . . . . . . . . . . . . . . . . . . . . . . . .

4.4 Representação somente dos elementos analíticos usados para representar o domínio a ser estudado. . . . . . . . . . . . . . . . . . . . . . .

4.5 Transformações de coordenadas lineares locais. A linha tem início em z1 e término em z2. A transformação para o plano $\mathrm{Z}$ leva para uma linha de início -1 e término 1 . . . .

4.6 Transformação de um círculo de raio r para uma elipse, ou para uma linha, caso r seja

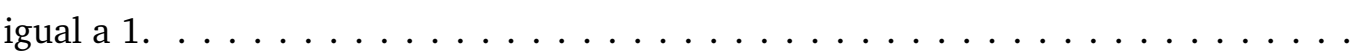

5.1 Representação da direção do escoamento uniforme de intensidade $Q_{0}$ e direção $\beta_{u f}$

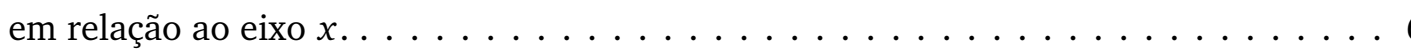

5.2 Comparação entre a expansão em Polinômios de Chebyshev e em Série de Potências, para $n=3$ 
5.3 Comparação entre a expansão em Polinômios de Chebyshev e em Série de Potências, para $n=15$. Nota-se que o Polinômio de Chebyshev preserva seu comportamento oscilatório no domínio, enquanto os termos para a Série de Potências são diferentes de zero apenas próximo às extremidades. . . . . . . . . . . . . 71

5.4 Lados do elemento onde o potencial $\Omega_{+}$e $\Omega_{-}$são considerados. . . . . . . . . . . 72

5.5 Esquema de uma fratura vertical comprimento $L$, altura $H$ e abertura $a$. Adaptado de

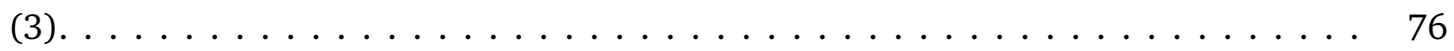

5.6 Esquema de uma fratura vertical com abertura $b$, condutividade hidráulica $K^{+}$, comprimento $L$ e altura $H$. Este é o formato da fratura utilizada neste trabalho. . . . . . 76

5.7 Fratura. Escoamento visto de dentro da fratura (esquerda) e do meio rochoso (direita). Adaptado de (4). . . . . . . . . . . . . . . . . . . . . . . . . . . . . 77

7.1 Esquema demonstrando a relação da direção com o escoamento uniforme e o domínio horizontal. . . . . . . . . . . . . . . . . . . . . . . . . . . . . 108

7.2 Comparação dos valores de carga hidráulica entre a solução exata e a obtida através do método iterativo implícito.

7.3 Valores de carga e linhas de corrente para a simulação da inomogeneidade circular calculado pelo método iterativo implícito. . . . . . . . . . . . . . . . . . . . . . 110

7.4 Inomogeneidades circulares com diferentes condutividades hidráulicas e raios, com o escoamento uniforme na direção $\frac{\pi}{6}$ radianos. Valores de carga hidráulica e linhas de corrente para o sistema com parâmetros na tabela $(7.2) \ldots \ldots$. . . . . . . . . . 111

7.5 Inomogeneidade circular formada por 20 line doublets. Valores de carga hidráulica e linhas de corrente para o sistema com parâmetros descritos na tabela (7.3). . . . . . . 113

7.6 Inomogeneidade circular poligonal composta por 20 line doublets (icoságono). Comparação de isolinhas de carga entre a solução aproximada da inomogeneidade circular poligonal e a solução exata para uma inomogeneidade circular. A concordância entre as duas soluções é aceitável, apesar de se notar erros próximo à borda do círculo. . . .

7.7 Inomogeneidade circular poligonal discretizada por 20 line doublets. Mapa de erro absoluto e relativo entre a solução exata e a solução aproximada pelo icoságono formado por line doublets. Os maiores erros ocorrem nos pontos de junção entre as line doublets. . . . . . . . . . . . . . . . . . . 
7.8 Inomogeneidade circular poligonal composta por 20 line doublets. Comparação das soluções geradas pelo algoritmo matricial e iterativo implícito para a inomogeneidade poligonal, com escoamento uniforme na direção $\pi / 3$ radianos. A concordância entre

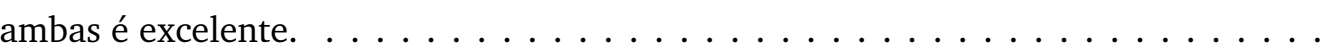

7.9 Duas inomogeneidades poligonais quadradas, com condutividades hidráulicas diferentes, em um campo de escoamento uniforme com ângulo de $\pi / 4$ radianos em relação ao eixo $x$. Valores de carga hidráulica e linhas de corrente. A inomogeneidade da direita tem condutividade hidráulica maior que a do meio, e a da esquerda, menor que a do meio. . . . . . . . . . . . . . . . . . . . . . . . . . . . . . . 117

7.10 Duas inomogeneidades poligonais em um campo de escoamento uniforme com ângulo de $\pi / 4$ radianos. Valores de carga para uma seção que passa através das duas inomogeneidades. Nota-se que o aumento da ordem do elemento diminui a descontinuidade do potencial, trazendo melhoria ao resultado. . . . . . . . . . . . . . . . . . . . . .

7.11 Solução analítica para uma fratura com condutividade hidráulica igual a 100 e abertura igual a 1, com comprimento igual a 100. Comparação entre os valores de carga para a fratura e uma elipse formada por 28 line doublets. A concordância é excelente. . . . . .

7.12 Solução analítica para uma fratura com condutividade hidráulica e abertura dadas pela tabela (7.9). Comparação com uma elipse formada por 28 line doublets e mesmo parâmetros do elemento fratura. O elemento fratura consegue modelar o escoamento, enquanto a line doublet não apresenta um resultado correto. . . . . . . . . . . . . . . . 121

7.13 Solução analítica para duas fraturas com condutividade hidráulica igual a $100 \mathrm{e}$ abertura igual a 1, com comprimento igual a 100. Comparação entre os valores de carga para as fraturas e duas elipse formada por 28 line doublets. A concordância também é excelente.

7.14 Solução usando o método matricial para duas fraturas com condutividade hidráulica e abertura dadas pela tabela (7.9). Isolinhas de carga hidráulica. Comparação com duas elipses formadas por 28 line doublets e mesmo parâmetros do elemento fratura. O elemento fratura consegue modelar o escoamento, enquanto a line doublet não apresenta um resultado correto. . . . . . . . . . . . . . . . .

7.15 Valores de carga e Linhas de corrente para o cruzamento de dois elementos analíticos para fratura.

7.16 Duas fraturas em cruzamento em um campo de escoamento uniforme a0 radiano. Comparação das isolinhas de carga hidráulica entre um sistema de line doublets e duas fraturaz em cruzamento. 
7.17 Sistema de fraturas em cruzamento em um campo de escoamento uniforme a $\pi / 4$ radianos. Os parâmetros estão relacionados na tabela (7.11). Valores de carga hidráulica. . . . . . . . . . . . . . . . . . . . . . . . . . 125

7.18 Sistema de fraturas em cruzamento em um campo de escoamento uniforme a $\pi / 4$ radianos. Linhas de corrente. Os parâmetros estão relacionados na tabela (7.11). . 125

7.19 Sistema de fraturas em cruzamento em um campo de escoamento uniforme a $\pi / 4$ radianos. Valores de carga hidráulica. . . . . . . . . . . . . . . . . . . . . . . 126

7.20 Sistema de fraturas em cruzamento em um campo de escoamento uniforme a $\pi / 4$ radianos. Linhas de corrente. . . . . . . . . . . . . . . . . . . . . . . . . . . . 127

7.21 Sistema de fraturas em cruzamento em um campo de escoamento uniforme a $\pi / 4$ radianos. Isolinhas de carga hidráulica e linhas de corrente, com os parâmetros dados pela tabela $(7.12) . \ldots \ldots \ldots \ldots \ldots \ldots$. . . . . . . . . . . . . . . 128

A.1 Esquema do experimento de Darcy. Adaptado de (1) . . . . . . . . . . . . . . . . . . 144

E.1 Gráfico gerado pelo Visu a partir da simulação SMEAgol. Isosuperfície de carga com três poços, line sinks e um campo de escoamento uniforme. . . . . . . . . . . . . . . . 152

E.2 Gráfico gerado no Visu a partir dos resultados do SMEAgol. Isolinhas de carga para um sistema com três poços, dois extratores e um injetor. . . . . . . . . . . . . . . . . . . 152

E.3 Gráfico gerado a partir do Visu e de um arquivo de dados com velocidades. Linhas de Corrente. . . . . . . . . . . . . . . . . . . . . . . . . . . . . . . . 152 


\section{Lista de Símbolos}

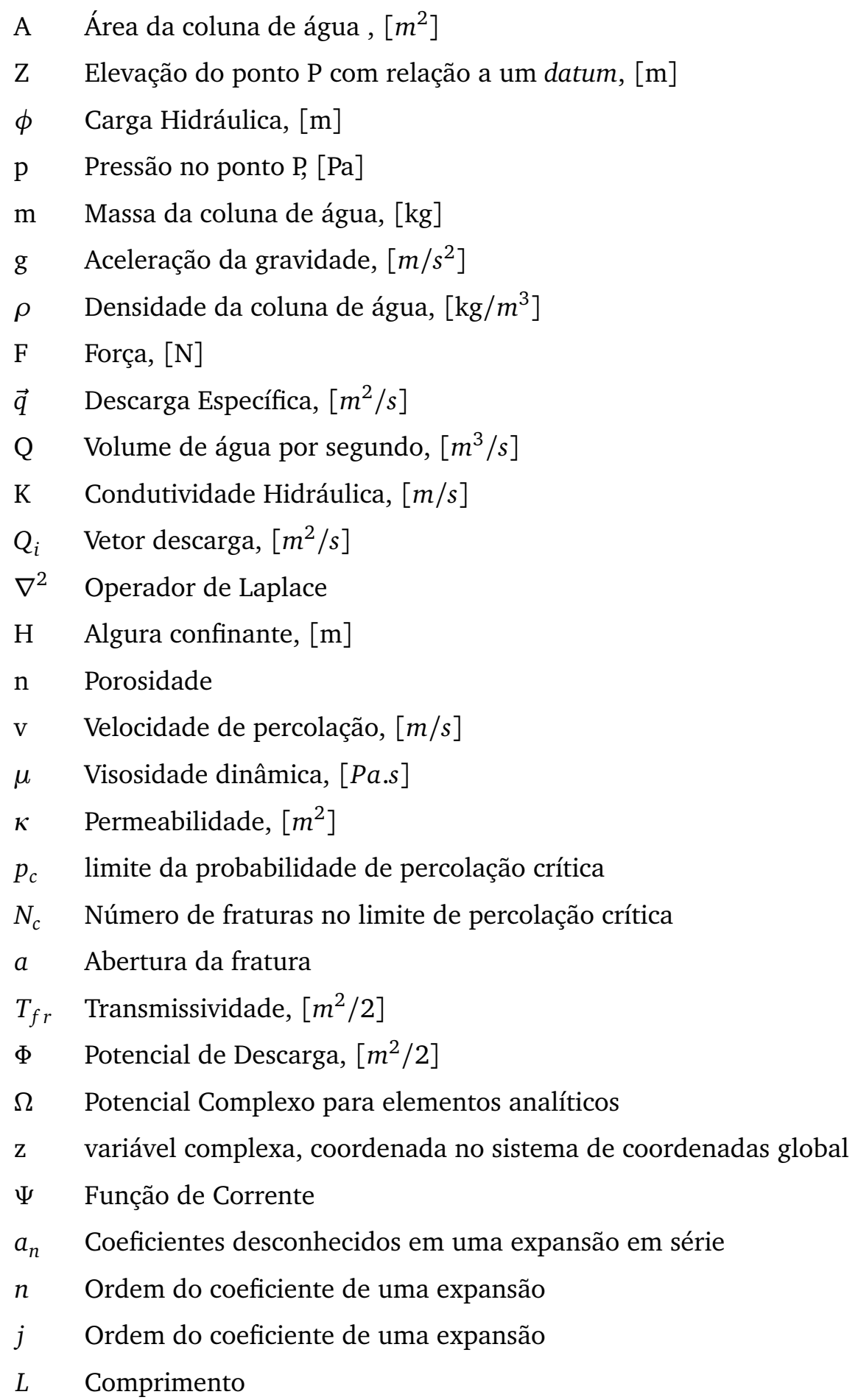


$\alpha \quad$ ângulo de orientação com relação ao eixo $x$

Z Coordenada local

$\chi \quad$ Coordenada após a transformação de Joukosky

$T_{n} \quad$ Polinômio de Chebyshev de primeiro tipo

$\lambda \quad$ Descontinuidade no potencial

$Q_{0} \quad$ Intensidade do escoamento regional, $\left[\mathrm{m}^{2} / \mathrm{s}\right]$

$\beta_{u f} \quad$ Direção do escoamento regional em relação ao eixo $x$

$R \quad$ Raio de uma inomogeneidade circular

$s \quad$ Intensidade de dipolo

$\beta_{j} \quad$ Coeficiente para expansão do para o infinito de uma line doublet

$\alpha_{j} \quad$ Coeficiente para expansão do campo distante de uma line doublet

$\sigma \quad$ influxo por unidade de comprimento em uma fratura, $\left[\mathrm{m}^{2} / \mathrm{s}\right]$

$(s, n) \quad$ Sistema de coordenadas local orientado na direção da fratura

W Vetor Descarga, complexo

$b^{*} \quad$ Abertura máxima de uma fratura

$H \quad$ Função analítica geral

d Coeficiente desconhecido para a expansão de $H$

$\neq j \quad$ Todos os outros coeficientes diferentes de $j$

$P_{i, k} \quad$ Coeficiente para elementos na diagonal da matriz

$R, T \quad$ Matrizes da partição para método iterativo

L Triângulo inferior de uma matriz, fora a diagonal

U Triângulo superior de uma matriz, fora a diagonal

D Diagonal de uma matriz

$e_{a b s} \quad$ Erro absoluto

$e_{\text {rel }} \quad$ Erro relativo

$e_{\text {rel/max }}$ Erro relativo máximo

$e_{a b s / \max } \quad$ Erro absoluto máximo

$n_{\text {elem }} \quad$ Número de coeficientes presentes na expansão em série de um elemento

$A_{v} \quad$ Espaço de Poros 


\section{Lista de Abreviações}

As abreviações nesta lista aparecem na ordem em que aparecem no texto.

MW MegaWatt, 1e6 Watts

WIPP Waste Isolation Pilot Plant

SPH Smoothed Particle Hydrodynamics

MEA Método dos Elementos Analíticos

REV Representative Elementar Volume

1D Uma dimensão espacial

2D Duas dimensões espaciais

3D Três dimensões espaciais

ADE Advection-Dispersion equation

CTRW Continuous Time Random Walk 


\section{Introdução}

A gama de contextos em que ocorre o escoamento de água subterrânea em meios porosos fraturados estende-se da captação de água e extração de petróleo à geração de energia, tanto geotérmica quanto em centrais nucleares. A captação e a contaminação da água são considerados grandes problemas para o século XXI (5-8), onde o uso de água subterrânea tem um papel de destaque. A geração de energia também é uma grande incógnita a ser resolvida, principalmente com os efeitos do aquecimento global (9-11). Dentre as formas conhecidas de geração de energia elétrica de carga de base, somente as gerações por centrais hidrelétricas, geotérmicas e nucleares têm baixa emissão de gases estufa (12-15). Apesar de ser uma tecnologia conhecida e testada nos últimos 50 anos, a geração de energia elétrica em centrais nucleares ainda é cercada por medos e mitos (12, 16-19), mesmo entendendo-se os riscos existentes. Acidentes como o de Windscale na Inglaterra (1957), Three Mile Island nos Estados Unidos (1979), Chernobyl na antiga União Soviética (hoje Ucrânia) (1986) e Fukushima Dai-ichi no Japão (2011) apenas aumentam estes receios, mesmo quando a literatura disponível sobre os efeitos da radiação proveniente desses acidentes não aponta a problemas de longo prazo (20) ou indica a necessidade de mais estudos para se obter uma conclusão sobre os problemas que a exposição à radiação podem causar (21). Com o acidente de Fukushima ainda em progresso e a ausência de estudos em profundidade sobre os reais impactos de tal acidente, suas consequências ainda são incertas, inclusive com respeito à contaminação dos aquíferos e o papel das fraturas em uma região sismicamente ativa. Mesmo se o uso da energia nuclear fosse abolido completamente, o descomissionamento dos reatores em funcionamento apresentaria um problema a ser resolvido.

Existem cerca de 400 reatores em operação no mundo gerando 370705 MW de potência elétrica (22). Tais reatores, dependendo do país, têm licença de operação até 2030 ou mais (22). O combustível nuclear usado, ao sair do reator, está quente, é altamente radioativo e tóxico. Uma primeira etapa de estocagem consiste em mantê-lo em piscinas de resfriamento, geralmente localizadas dentro do prédio de contenção do reator. Após este período, que pode durar até 4 anos, uma destinação final deve ser dada, sendo que as opções vão de reprocessamento à disposição definitiva. Mesmo que o reprocessamento seja executado, parte do material, devido a sua toxicidade, precisa ser armazenado de forma definitiva. 
Uma das soluções para o armazenamento do combustível usado e de materiais provenientes do descomissionamento de reatores nucleares é o uso de repositórios geológicos (15, 23-29). O uso de repositórios geológicos demanda, por sua própria natureza, de um entendimento do escoamento da água subterrânea, especialmente se o meio for fraturado, como geralmente é o caso.

\subsection{Repositórios geológicos}

Existem diversas soluções propostas para tratar resíduos nucleares e combustível usado, entre elas (29): disposição extraterrestre, transmutação em outros elementos menos tóxicos, submerção de contêineres no mar ou na Antártida, ou, ainda, afundar em áreas de subducção da crosta. As soluções presentemente em uso são estocagem seca em caskets nos sítios das usinas, estocagem dentro das piscinas de resfriamento dentro do próprio reator, e repositórios geológicos, como o Waste Isolation Pilot Plant (WIPP), nos Estados Unidos (30).

Para gerenciar o combustível usado, o conceito de reservatório geológico vem sendo estudado para diferentes cenários $(23,31)$. Para a escolha do local de construção e futura operação segura de um repositório geológico, os impactos ambientais devem ser analisados, levando em conta a geologia e a hidrogeologia do local escolhido, em escalas de tempo suficientemente longas, considerando os tempos de decaimento dos materiais a serem armazenados. O estudo da hidrogeologia da região é fundamental para assegurar que a construção e uso do reservatório seja seguro para os aquíferos presentes, e que a instalação não irá prejudicar os recursos de água subterrânea, que inclusive é o principal vetor de migração do material estocado no repositório $(27,29)$.

Nesse contexto, o entendimento do escoamento em meios porosos fraturados é essencial $(4,26)$, já que o escoamento nas fraturas pode ser ordens de magnitude mais rápido que o do meio poroso $(26,32)$, e consequentemente pode transportar solutos de maneira muito mais rápida de volta à superfície (32).

\subsection{Escoamento em Fraturas}

O escoamento de água subterrânea em meios porosos fraturados é um problema que tem recebido muita atenção a partir da década de 1970 (23, 24), em parte devido ao problema dos repositórios geológicos, apesar de ser um problema de interesse da área de extração de petróleo e energia geotérmica, além de produção de água para consumo $(32,33)$. O problema de meios fraturados é complexo, com fenômenos em diferentes escalas interagindo entre si, onde a geometria das fraturas influenciam no escoamento e transporte. A obtenção de dados experimentais para os sistemas fraturados é difícil.

As abordagens para simular o escoamento em fraturas são dificultadas pelas características do 
método numérico escolhido. Elementos finitos e diferenças finitas, apesar de serem capazes de representar propriedades de fraturas, apresentam problemas em simular sistemas fraturados em que a variação de escala no tamanho das diversas fraturas presentes é relevante. Nesses casos, uma discretização de malha para fraturas de comprimento de $1 \mathrm{~m}$ dificilmente seria computacionalmente viável ao se incluir no mesmo sistema fraturas da ordem de $1 \mathrm{~km}$ (34). Métodos como Lattice Boltzmann (35) e SPH (36) conseguem reproduzir bem os efeitos das paredes como rugosidade e tortuosidade, mas somente em dimensões microscópicas.

O Método de Elementos Analíticos (MEA) (1), por não necessitar de discretização do domínio e usar soluções analíticas para a equação governante, permite a simulação de características hidrogeológicas em diferente escalas, desde que um elemento analítico (uma solução da equação governante) para tais características exista. Apesar das condições de contorno para fraturas no MEA já terem sido estabelecidas, e uma solução analítica para uma fratura isolada existir na literatura (4), uma generalização para diversas fraturas interagentes entre si e interagindo com o meio poroso não foi apresentada.

\subsection{Modelagem Numérica}

A modelagem numérica de fenômenos hidrogeológicos envolve diversos campos da Ciência (37). O fenômeno físico é descrito em um modelo matemático. O modelo matemático é aproximado por um método numérico, de onde resultados são extraídos e comparados com dados existentes sobre o fenômeno. A modelagem numérica é uma ferramenta prática para se obter informações e entendimento sobre fenômenos complexos. Custo, problemas de escala ou reprodutibilidade, além da falta de dados físicos suficientes de entrada são algumas das limitações para as quais a aplicação de um modelo numérico é indicada. Entretanto, um modelo numérico pode em muitos casos não reproduzir a realidade, tanto pela falta de dados de entrada, como pela falha do próprio modelo, ou pela interpretação errônea dos resultados do modelo.

A modelagem numérica de um fenômeno físico, como o escoamento de água subterrânea, consiste na criação de ferramentas computacionais usadas na solução das equações governantes do modelo matemático de tal fenômeno (37). A implementação do modelo numérico é geralmente, feita em um computador. Computadores, devido a sua própria natureza, apresentam a limitação de apenas poderem trabalhar com representações discretas e finitas de grandezas contínuas, ao contrário de modelos matemáticos e físicos, que podem incluir grandezas tanto contínuas como discretas. Essa implementação pode dar origem a erros, como o erro de truncagem e o erro de aproximação, como pode ser visto na seção (6.9). O trabalho de um construtor de modelos numéricos é minimizar a diferença entre a realidade física e o modelo numérico implementado, e considerar ao mesmo tempo o custo computacional necessário para tal fim. O balanço entre a precisão do modelo e o custo do mesmo é delicado, já que em certos casos o custo computacional para uma solução ter acurácia 
aceitável a proíbe de ser útil.

\subsection{O Método de Elementos Analíticos - MEA}

O Método de Elementos analíticos foi desenvolvido para simular o escoamento de água subterrânea em escala regional (38). O método usa soluções da equação governante do escoamento $(1,38)$, satisfazendo as condições de contorno internas de forma aproximada. Cada elemento hidrogeológico (como um rio ou um poço) é representado por uma solução da equação governante, chamado de elemento analítico, com uma condição de contorno que deve ser imposta sobre cada elemento, caracterizando uma condição de contorno interna. As soluções para a equação governante são exatas, mas as condições de contorno são satisfeitas apenas aproximadamente (39). Outra característica marcante do MEA é que ele não requer que o domínio seja limitado por fronteiras, sendo necessária apenas a condição de contorno no infinito(40) e as condições internas em determinados pontos.

O MEA vem sendo desenvolvido há mais de 20 anos, com sua aplicação focada em água subterrânea, sem uma generalização para outras áreas, como o que ocorreu com o método de elementos finitos e diferenças finitas. Apesar disto, diversas aplicações do MEA são usadas no mundo, principalmente por agências reguladoras para monitoramento de grandes bacias e para simulações em grandes domínios (41-44). Novos elementos ainda são desenvolvidos e avanços na generalização do método são feitas tanto por Otto D. L. Strack, que foi o pioneiro em seu desenvolvimento (43), como pela comunidade (45-47).

\subsection{Este Trabalho}

O objetivo deste trabalho é desenvolver um elemento analítico para um sistema de fraturas baseado no Método de Elementos Analíticos, aplicando novas técnicas para satisfazer as condições de contorno impostas para as fraturas e outros elementos. No desenvolvimento do mesmo, o método iterativo implícito apresentou problemas de ordem numérica. Na tentativa de sanar tais problemas, foi desenvolvimento de um método matricial, usando elementos do desenvolvimento recente para métodos iterativos. Este método matricial foi generalizado para todos os elementos aqui descritos. Uma análise dos métodos iterativos implícitos usados recentemente (48) no Método de Elementos Analíticos foi feita, demonstrando que a convergência dos mesmos não é garantida.

Este trabalho está organizado da seguinte forma: no capítulo (2) uma rápida introdução nos conceitos de Hidrogeologia usados é feita. No capítulo (3) é apresentado um panorama geral da literatura sobre o problema do escoamento de água em meios fraturados, com uma demonstração da lei de placas paralelas. No capítulo seguinte (4), uma revisão do Método de Elementos Analíticos é efetuada, dando o embasamento para o desenvolvimento dos elementos no capítulo (5), onde os 
elementos simulados - a inhomogeneidade circular, a inhomogeneidade poligonal usando line doublets e a fratura - são apresentados e as condições de contorno para cada um são mostradas. O capítulo (6) descreve a implementação numérica dos elementos, mostrando os algoritmos iterativo implícito, matricial e iterativo explícito. Os resultados são apresentados no capítulo (7) e as conclusões no capítulo (8).

Durante o desenvolvimento deste trabalho houve uma intensa colaboração com o Prof. Otto Strack, da University of Minnesota, onde as novas técnicas para as inomogeneidades circulares e as line doublets foram estudadas, aplicadas e generalizadas para as fraturas. O Prof. Otto Strack também possibilitou o uso do Relatório Battelle (4), de onde a solução analítica para uma fratura foi obtida. 


\section{Hidrogeologia}

Neste trabalho, o escoamento de água subterrânea é considerado estacionário, incompressível, laminar e irrotacional. Tais características permitem diversas simplificações ${ }^{1}$ no tratamento do problema.

\subsection{Introdução}

Pode-se encontrar água em quase todos os ambientes terrestres sobre ou próximo da superfície terrestre. Água pode ser encontrada na superfície, na forma de rios, lagos e mares, em geleiras nos pólos, e no subsolo, como água subterrânea. O Ciclo Hidrológico é o fenômeno global de circulação fechada de água entre a subsuperfície, superfície terrestre e a atmosfera, impulsionado fundamentalmente pela energia solar, associada à gravidade e à rotação terrestre(49). Hidrogeologia consiste no estudo da água nos espaços porosos da subsuperfície $(50,51)$.

\subsection{Definições de Hidrogeologia}

Rochas contém poros, fraturas e fissuras. Estes espaços contém ar ou água, ou outro fluido, como gás ou petróleo. Os poros devem ser conectados entre si para permitir a passagem do fluido (50).

Para um aquífero não-confinado, o perfil vertical da água subterrânea pode ser dividido em zonas. Na zona saturada ou freática todos os poros estão ocupados por água, a partir de um leito rochoso impermeável, sendo limitada superiormente pela superfície chamada de superfície freática. A pressão na superfície freática é igual à pressão atmosférica.

Na zona insaturada, ou zona vadosa, os poros estão parcialmente preenchidos com água, ar ou vapor d'água. Esta zona é afetada pelas condições da superfície: chuva, seca, e variações sazonais ou diárias. Água se move para baixo - infiltração - devido à gravidade e para cima por evapotranspiração.

Um aquífero é uma formação geológica que se distingue de outras camadas por conter água e por poder produzir água em quantidades significativas em condições ordinárias de campo $(49,51)$.

\footnotetext{
${ }^{1}$ como o uso da teoria do potencial e variáveis complexas.
} 
infiltração é o nome dado ao processo da água penetrar na camada superficial do solo, que pode evaporar ou dar origem à recarga. Recarga é o processo de percolação da água infiltrada até a zona saturada. A água que precipita sobre a superfície mas não infiltra dá origem ao escoamento superficial.

As dimensões horizontais de um aquífero geralmente são muito maiores que as dimensões verticais, o que possibilita algumas aproximações. O aquífero pode ser confinado, quando existe uma camada impermeável confinante na superfície do aquífero, ou não confinado ou livre, onde a pressão na superfície freática é a pressão atmosférica.

Água subterrânea é água encontrada na zona saturada.

\subsection{O Meio Poroso}

Poro é um espaço vazio em meio a um material sólido (52). Solos, rochas porosas, rochas fissuradas, cerâmicas são materiais porosos. Um material poroso é constituído por duas ou mais fases, sendo uma sólida e as outras líquidas ou gasosas. A parte sólida é chamada de matriz sólida (matriz rochosa no caso de rochas), e a parte preenchida por líquido, gases ou vazia é chamada de espaço vazio ou espaço de poros. Como a geometria dos poros é difícil de ser estabelecida, considerando o tamanho dos domínios de interesse deste trabalho, além da imensa dificuldade de se descrever o escoamento de moléculas de água individuais neste sistema, uma aproximação para um meio contínuo é geralmente usada (52).

\subsubsection{A Aproximação Contínua para Meios Porosos}

O fluido, em escala nanoscópica, é composto por uma grande quantidade de moléculas em movimento, sujeitas a forças moleculares (53), sofrendo colisões com as paredes e outras moléculas. Uma descrição determinística do comportamento das moléculas do fluido é uma empreitada sem esperança: mesmo em uma abordagem numérica, a simulação de $10^{23}$ moléculas em um grama de água é impraticável. E mesmo que a descrição de todas as moléculas e as forças que nelas atuam fosse factível, a tarefa de determinar a posição e o momento linear inicial de cada molécula é impossível, por exemplo, por métodos de observação disponíveis no presente. Entretanto, sistemas com grande quantidade de graus de liberdade fornecem uma saída para sua descrição: o uso de Mecânica Estatística (54). Sistemas com grande quantidade de graus de liberdade, como um fluido, obedecem as mesmas leis da Mecânica que sistemas com poucas partículas. Entretanto, o número de graus de liberdade possibilita uma descrição física que permite a predição e dedução dos fenômenos macroscópicos. Essa descrição de sistemas macroscópicos é uma descrição estatística e probabilística. A característica probabilística da descrição dos sistemas não é uma característica dos sistema e dos componentes considerados, mas surge do fato que os resultados são derivados de muito menos informação do que seria necessário para uma completa descrição mecânica do mesmo. 
Ao abandonar-se a descrição microscópica e utilizar-se uma descrição estatística, o fluido pode ser tratado como um meio contínuo. Para o uso da descrição contínua, define-se uma partícula do fluido. Uma partícula de fluido é um ensemble ${ }^{2}$ de moléculas em um pequeno volume. O tamanho da partícula é muito maior que o caminho livre médio ${ }^{3}$ das moléculas, mas é pequena o suficiente em relação ao volume total do fluido. Medidas para uma partícula fornecem resultados para as propriedades físicas relevantes. Com esta transição, foi-se da descrição molecular de um fluido para a descrição microscópica do mesmo, onde o fluido é um meio contínuo confinado pelas paredes do meio poroso. Neste nível é possível usar a Mecânica de Fluidos, descrevendo o movimento do fluido viscoso através das equações de Navier-Stokes. Entretanto, tal descrição é praticamente impossível em água subterrânea, dada a complexa geometria do espaço de poros e a dificuldade inerente em se obter tal geometria. Uma solução é descrever o meio poroso mais fluido como um contínuo em escala macroscópica na forma de um campo médio.

Para esta média estatística definir o sistema macroscópico, um volume elementar representativo (Representative Elementar Volume, REV) (26) deve ser menor que o domínio de interesse, mas grande o suficiente para incluir um número de poros que permita que a média estatística tenha significado físico na abordagem contínua.

\subsubsection{A Aproximação Contínua Para O Meio Fraturado}

Existem, entretanto, características da subsuperfície que dificultam a aproximação contínua. Um exemplo destas perturbações são fraturas, presentes em quase todos os meios rochosos. Fraturas são descontinuidades em um bloco rochoso, composta por espaçamentos, falhas, junções, rachaduras e fissuras $(26,32)$, com causas diversas. A presença de fraturas e sua grande gama de variabilidade em comprimento, abertura e rugosidade podem impedir a correta descrição do sistema macroscópico em um REV (26). Existem diversas abordagens na literatura $(26,32,33)$ para levar esta e outras dificuldades em conta, e são descritas em detalhe no capítulo 3.

\subsection{Definições Matemáticas}

\subsubsection{Carga Hidráulica}

A carga hidráulica em um ponto $P$ do subsolo é definida como o nível que a água atinge em um tubo aberto com sua ponta inferior a $P$. É um nível, e deve ser definido em relação a um ponto de referência, o datum. Datum é o nível de referência adotado para o modelo, a partir de uma

\footnotetext{
${ }^{2}$ ensemble é o termo que em Física descreve uma coleção de vários sistemas com as mesmas propriedades estatísticas

${ }^{3}$ Caminho livre médio é a distância que uma molécula de água percorre antes de colidir com outra molécula.
} 


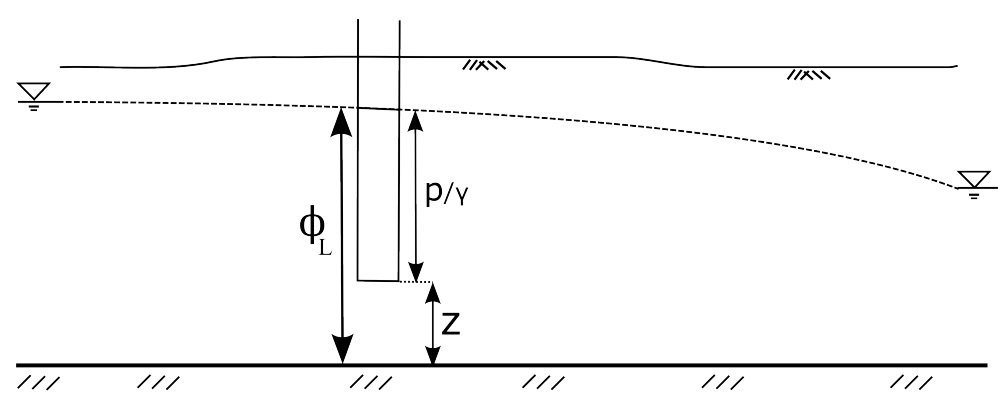

Figura 2.1 - Esquema de um piezômetro em um aquífero livre. $\phi$ é a carga hidráulica, $\frac{p}{\gamma}$ é a carga de pressão e $z$, a carga de elevação.

representação teórica da superfície da Terra ${ }^{4}$. Dada uma coluna de água de área $A$ e altura $\phi-Z$, onde $Z$ é a elevação do ponto $P$ em relação ao datum e $\phi$ a altura do nível da água em relação ao datum, com $g$ a aceleração local da gravidade e $\rho$ a densidade da água, a pressão $p$ no ponto $P$ é igual a

$$
p=\frac{F}{A}
$$

e pela Segunda Lei de Newton,

$$
F=m g
$$

A massa acima do ponto $P$ é igual a

$$
m=(\phi-Z) A \rho
$$

Portanto, usando a definição de pressão,

$$
P(x, y)=\frac{F}{A}=\rho g(\phi-Z)
$$

ou seja, $\phi$ é definido como

$$
\phi(x, y, z)=\frac{P}{\rho g}+Z
$$

onde o termo $\frac{P}{\rho g}$ é chamado carga de pressão e Z carga de elevação ou posição. $\phi(x, y, z)$ é a chamada carga hidráulica em escoamento de água subterrânea, como pode ser visto na figura (2.1).

\subsubsection{Lei de Darcy}

A Lei de Darcy relaciona a carga $\phi(x, y, z)$ à descarga específica $\vec{q}(x, y, z)$. A Lei de Darcy foi primeiramente deduzida para escoamento retilíneo de um fluido homogêneo em meio poroso. É uma lei experimental, assim como a lei de Ohm e Fourier, e relacionada a esta. A experiência original Terra.

${ }^{4} \mathrm{O}$ termo datum se refere aos pontos de referência a partir dos quais se fazem medidas na superfície da 
é descrita, por exemplo, em (1), e consiste em medir a diferença de nível - carga hidráulica - da entrada e da saída de um tubo preenchido com material poroso que gera o deslocamento da água:

$$
\begin{array}{r}
q=k \frac{\left(\phi_{1}-\phi_{2}\right)}{L} \\
Q=A k \frac{\left(\phi_{1}-\phi_{2}\right)}{L}
\end{array}
$$

onde $Q$ é o volume de água por segundo a escoar no sistema. A descarga específica $q$ é o volume de água $\left(L^{3}\right)$ que escoa por unidade de área $\left(L^{2}\right)$ por unidade de tempo $(T)$ :

$$
q \frac{[L]}{[T]}
$$

A expressão (2.6) pode ser generalizada para uma relação diferencial:

$$
Q=-K A \frac{d \phi}{d x}
$$

Para problemas tridimensionais, a lei de Darcy também pode ser descrita como uma relação vetorial:

$$
\vec{q}(x, y, z)=-K \vec{\nabla} \phi(x, y, z)
$$

em que o gradiente representa a direção da maior taxa de aumento do campo escalar, com a magnitude igual à taxa de aumento. A descarga específica $\vec{q}(x, y, z)$ pode ser descrita como um campo vetorial Em notação tensorial:

$$
q_{i}=-K \partial_{i} \phi
$$

A Lei de Darcy, juntamente com a equação de continuidade descrita em (2.4.4), descrevem a dinâmica de escoamento de fluidos em meios porosos, dada a condição de um escoamento laminar ${ }^{5}$.

\subsubsection{Vetor Descarga e o Potencial de Descarga}

O vetor descarga, $Q_{i}\left(L^{2} / T\right)$, é igual à integração do vetor de fluxo específico na direção vertical:

$$
Q_{i}=\int_{0}^{h} q_{i} d z
$$

onde $h$ é a altura saturada do aquífero não confinado. $Q_{i}$ equivale à descarga por unidade de largura na direção $i\left(L^{3} / T\right)$.

\footnotetext{
${ }^{5}$ ou seja, número de Reynolds baixo. Mais detalhes sobre o domínio de validade da Lei de Darcy podem ser encontrados em $(1,52,55)$. Uma discussão sobre a validade da Lei de Darcy para meios fraturados pode ser encontrado no capítulo 3 e em (26)
} 
Para um aquífero confinado, a relação da descarga com fluxo específico é igual a

$$
Q_{i}=H q_{i}
$$

em que $H$ é a espessura do aquífero confinado. Pode-se definir o vetor de descarga em função da carga hidráulica e da lei de Darcy, obtendo-se

$$
Q_{i}=H q_{i}=H\left(-K \frac{\partial \phi}{\partial x_{i}}\right) Q_{i}=\left(-\frac{\partial H K \phi}{\partial x_{i}}\right) .
$$

Se o meio for isotrópico e homogêneo, pode-se definir o vetor de descarga $Q_{i}$ como o gradiente de um potencial. Considerando $H$ e $K$ como constantes para um determinado aquífero, define-se um potencial de descarga $\Phi$ igual ao termo dentro da derivada parcial adicionada uma constante $C_{C}$, na forma:

$$
\Phi=K H \phi+C_{C}
$$

Assim, o vetor de descarga pode ser definido como

$$
Q_{i}=-\frac{\partial \Phi}{\partial x_{i}}
$$

Na superfície freática, a carga hidráulica $\phi(x, y, z)$ é igual à espessura saturada $h(x, y)$. O vetor descarga neste caso é igual a

$$
Q_{i}=\phi q_{i}
$$

Com a aplicação da Lei de Darcy, obtém-se:

$$
Q_{i}=-K \phi \frac{\partial \phi}{\partial x_{i}}
$$

ou, usando o fato que $K$ é uma constante,

$$
Q_{i}=-\frac{\partial \frac{1}{2} K \phi^{2}}{\partial x_{i}}=-\frac{\partial}{\partial x_{i}}\left[\frac{1}{2} K \phi^{2}\right]
$$

O potencial de descarga para o caso de escoamento não-confinado é definido como

$$
\Phi(x, y, z)=\frac{1}{2} K \phi^{2}+C_{L} .
$$

Em sistemas onde existem regiões confinadas e não confinadas, uma única forma de potencial pode ser escrita para todo o sistema, definindo as constantes das equações (2.14) e (2.19) e dos potenciais de acordo com a condição de continuidade do potencial na transição entre região confinada e não confinada. A figura (2.2) mostra a transição de uma região confinada para uma região não 
confinada. O potencial da região confinada fica igual a

$$
\Phi(x, y, z)=K H \phi-\frac{1}{2} K H^{2}
$$

onde as constantes $C_{C}$ e $C_{L}$ foram definidas como

$$
C_{C}=-\frac{1}{2} K H^{2}
$$

e

$$
C_{L}=0
$$

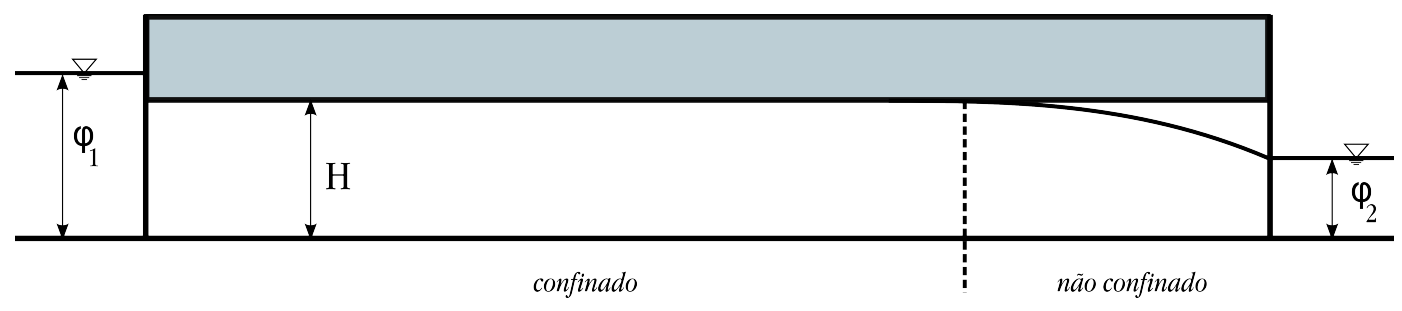

Figura 2.2 - Escoamento unidimensional com transição do regime confinado para o regime não confinado. Adaptado de (1).

\subsubsection{Equação da Continuidade e a Equação de Laplace}

O potencial $\Phi$ definido desta forma facilita a descrição de problemas para regiões confinadas e regiões não-confinadas, além de permitir que a equação de Laplace para o potencial seja sempre linear. O escoamento de água obedece a equação de continuidade: para um bloco de rocha saturado não há criação ou armazenamento de água, e o balanço de entrada e saída deste bloco deve ser igual a zero. Equacionando os fluxos de entrada e saída do bloco, obtém-se que a equação de continuidade é igual a

$$
\begin{array}{r}
\Delta Q=0= \\
-\sum_{i j k} \Delta x_{i} \Delta x_{j}\left[q_{k}\left(x_{i}, x_{j}, x_{k}+\frac{\Delta x_{k}}{2}\right)-q_{k}\left(x_{i}, x_{j}, x_{k}-\frac{\Delta x_{k}}{2}\right)\right] \frac{(-1)^{\epsilon_{i j k}}}{2} \epsilon_{i j k}
\end{array}
$$

Dividindo por $\Delta x_{i} \Delta x_{j}$ e fazendo o limite dos termos $\Delta x_{i} \rightarrow 0$, obtém-se 


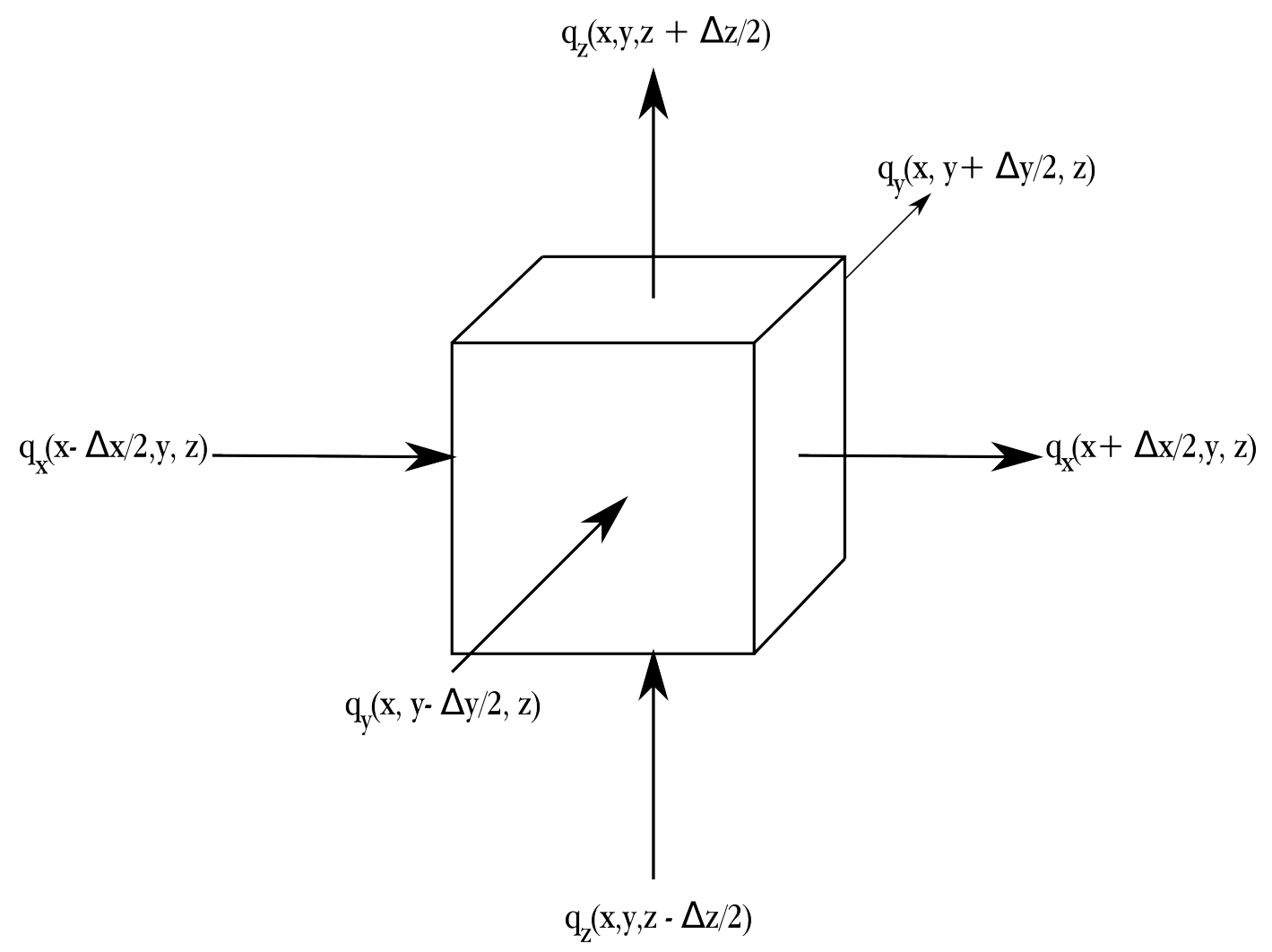

Figura 2.3 - Esquema do vetor de descarga específica para um bloco. Adaptado de (1).

$$
\frac{\partial q_{x}}{\partial x}+\frac{\partial q_{y}}{\partial y}+\frac{\partial q_{z}}{\partial z}=0
$$

ou, em notação tensorial,

$$
\partial_{i} q_{i}=0
$$

A figura (2.3) detalha as componentes da descarga específica entrando e saindo do bloco. Como a condutividade hidráulica é considerada como homogênea por partes e isotrópica, pode-se aplicar a lei de Darcy e obter-se a equação de Laplace em termos da carga para o caso confinado:

$$
\frac{\partial^{2} \phi(x, y, z)}{\partial x^{2}}+\frac{\partial^{2} \phi(x, y, z)}{\partial y^{2}}+\frac{\partial^{2} \phi(x, y, z)}{\partial z^{2}}=0
$$

Para o caso não-confinado a equação de Laplace em função da carga hidráulica torna-se não-linear. Substituindo a equação (2.18) na equação de continuidade, a equação de Laplace para o caso não-confinado é igual a

$$
\frac{\partial^{2} \phi^{2}(x, y, z)}{\partial x^{2}}+\frac{\partial^{2} \phi^{2}(x, y, z)}{\partial y^{2}}+\frac{\partial^{2} \phi^{2}(x, y, z)}{\partial z^{2}}=0
$$


que é não linear, justificando o uso do potencial (2.19). A equação de Laplace para o potencial é igual a

$$
\frac{\partial^{2} \Phi(x, y, z)}{\partial x^{2}}+\frac{\partial^{2} \Phi(x, y, z)}{\partial y^{2}}+\frac{\partial^{2} \Phi(x, y, z)}{\partial z^{2}}=0
$$

Esta é a equação governante que será usada neste trabalho, que serve tanto para o caso confinado quanto o não-confinado. Todas as características hidrogeológicas a serem descritas são solução da equação de Laplace tridimensional, ou bidimensional quando a aproximação de Dupuit-Forchheimer (52) é usada.

Porosidade é definida como a relação entre o volume de vazios (poros) e o volume total da região considerada:

$$
n=\frac{V_{p}}{V_{t}}
$$

onde $V_{p}$ é o volume de vazios (poros) do meio rochoso, e $V_{t}$ é o volume total da amostra. A velocidade de percolação é igual a

$$
v=\frac{q}{n_{e q}}=\frac{Q L}{A t}
$$

onde $q$ é a descarga específica ou velocidade de Darcy e $n_{e q}$ a porosidade efetiva, que contabiliza somente os poros conectados. Ao se usar a área de rocha e área de espaços (poros) a velocidade de percolação é igual a

$$
v=\frac{Q}{A_{v}}
$$

onde $Q$ é a vazão ou descarga de água por unidade de tempo:

$$
Q, \frac{\left[m^{3}\right]}{[s]}
$$

Permeabilidade é uma propriedade do meio poroso, enquanto condutividade hidráulica é uma propriedade do sistema meio poroso mais fluido. A relação entre a permeabilidade e a porosidade é dada por

$$
K=k \frac{\rho g}{\mu}
$$

onde $k$ é a permeabilidade, $K$ a condutividade hidráulica, $\mu$ a viscosidade do fluido, $\rho$ a densidade e $g$ a aceleração gravitacional média. A unidade da permeabilidade é $m^{2}$ ou darcy, que equivale a $1 D=10^{-12} \mathrm{~m}^{2}$. 


\section{Descrição e Modelagem de Fraturas}

Neste capítulo é feita uma revisão da literatura para o problema de escoamento de água subterrânea e transporte de solutos em meios porosos fraturados. O capítulo é iniciado com uma revisão geral para escoamento em fraturas, com uma breve descrição dos mecanismos de transporte, terminando com o modelo de placas paralelas. O modelo de fraturas que obedecem uma relação linear com a abertura é descrito ao final do capítulo.

\subsection{Características de Fraturas}

Fraturas são rupturas na crosta terreste ou de corpos rochosos sem que haja deslocamento dos blocos resultantes. Falhas geológicas ou somente falhas são fraturas planares em rochas onde houve deslocamento de um bloco em relação à outro. Estes deslocamentos podem ser de poucos centímetros a dezenas de quilômetros.

Redes de falhas geralmente são compostas por duas ou três famílias distintas de fraturas orientadas. Junções, ao contrário, podem ser compostas por uma infinidade de famílias de fraturas, potencialmente não distinguíveis. Junções geralmente são mais conectadas e densas espacialmente. Falhas não são tão bem conectadas, frequentemente como consequência da densidade e orientação das mesmas. Neste trabalho o termo fratura será usado indistintamente para ambos os casos. De forma geral, as fraturas são caracterizadas por

- Geradas por vários fenômenos como tectonismo, erosão, ou subsidências de acomodação de terreno

- Grandes diferenças de escala - variações de centrímetros a quilômetros

- Incertezas nas medidas das propriedades (e até mesmo na definição das propriedades)

- Escoamento e transporte em escalas de tempo e espaço diferentes

Diversos parâmetros podem ser usados para caracterizar fraturas individuais. Entre os principais estão $(56,57)$ : 
- Rugosidade na parede da fratura

- Abertura

- Comprimento

- Orientação

- Ângulo de mergulho

Além dos descritos acima, outros parâmetros são usados para caracterizar conjuntos de fraturas, como $(26,32,57)$ :

- Densidade

- Conectividade

- Escalabilidade

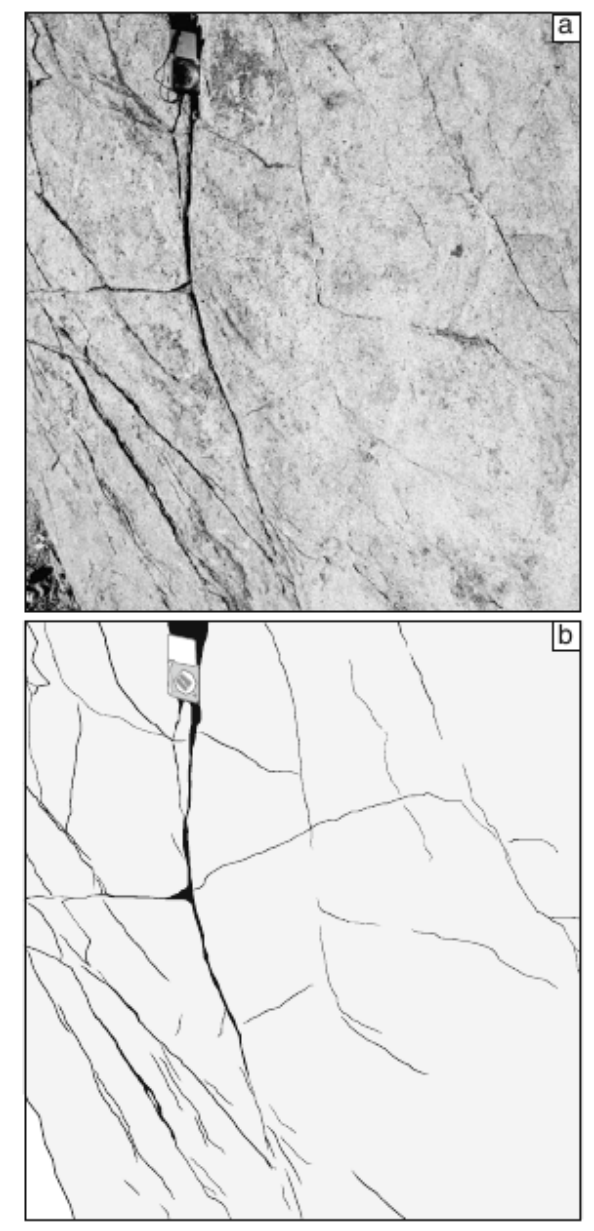

Figura 3.1 - Reproduzida de Klimczak et al. (2). Perfil de uma fratura em um afloramento rochoso. A figura $a$ é uma fotografia, enquanto a figura $b$ é um desenho feito por um geólogo evidenciando o sistema de fraturas. 
(a)

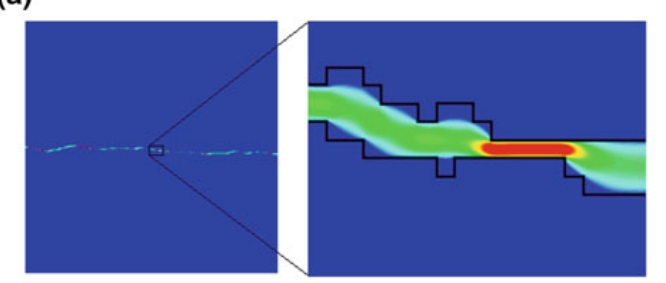

(b)

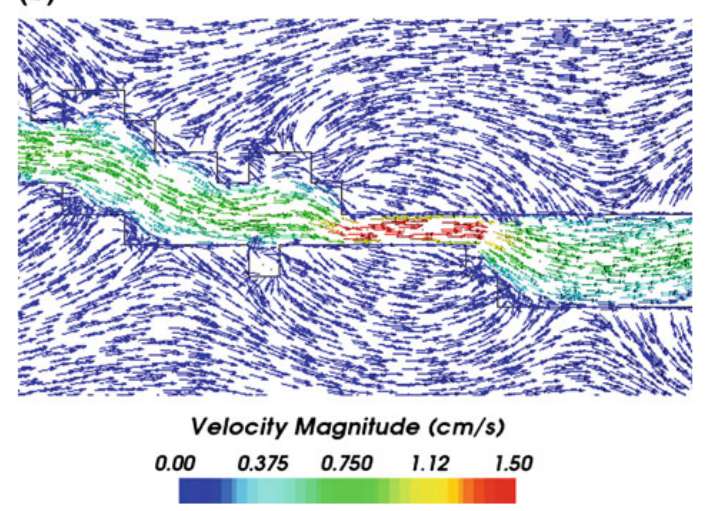

Figura 3.2 - Reproduzida de Crandall et al. (3). Simulação de escoamento usando elementos finitos em uma fratura com paredes rugosas. Diferença de pressão entre o lado esquerdo e direito de $\Delta P=100 P a$, Permeabilidade do meio poroso $\kappa=200 \mathrm{md}$. Comprimento da fratura: $10 \mathrm{~cm}$. Permeabilidade da fratura $\kappa=362 m d$, onde $m d$ denota $1 e^{-3}$ darcys.

A figura (3.1) mostra um afloramento rochoso fraturado. O desenho $b$ de (3.1) evidencia o padrão das fraturas, mostrando uma fratura principal no sentido vertical com fraturas de abertura aparente menores intersectando com a no sentido vertical. Na figura (3.2), da referência (3), mostra uma simulação de escoamento usando elementos finitos em uma reconstrução de uma fratura a partir de um bloco rochoso. O gráfico evidencia a influência da rugosidade no escoamento na fratura e na interação do escoamento entre a fratura e o meio poroso. É usual na área distinguir entre rochas fraturadas e rochas porosas fraturadas (26). No primeiro caso, o fluxo é considerado como ocorrendo somente nas fraturas. No segundo, o meio poroso é incluído na descrição do escoamento e na modelagem. Outra característica importante na descrição de fraturas é o fator de escala, já que os processos que ocorrem podem ser dependentes da escala, ou podem interagir entre as diferentes escalas $(32,58)$. Ao se descrever um sistema fraturado deve-se sempre ter em mente a escala do problema de interesse e, correspondentemente, o nível de detalhe na descrição do mesmo. Fraturas podem apresentar abertura variável ao longo do seu comprimento, com paredes de rugosidade variável e possível deposição de minerais e ocorrência de reações químicas em suas paredes.

\subsubsection{Características Físicas e Geométricas}

A descrição geofísica é fundamental para a modelagem de fraturas, particularmente para o estudo do fluxo de água subterrânea, já que fraturas podem ser canais preferenciais para o escoamento (32). A análise de fraturas em campo é altamente custosa, já que são de difícil acesso, ocorrem em uma grande faixa de formações geológicas e diferentes tipos de rocha. Fraturas têm suas 
dimensões variando de microfraturas na escala de poucos micrômetros a grandes fissuras da ordem de quilômetros de comprimento, e sua estrutura geométrica varia de arranjos poligonais relativamente ortogonais a padrões randômicos de distribuição espacial. As propriedades hidráulicas e de transporte também variam grandemente, dependendo do grau de interconexão entre as fraturas, variações na abertura e características químicas das fraturas e da rocha hospedeira (59).

Dados experimentais sobre fraturas geralmente são obtidos usando as seguintes dados $(26,32$, 60):

- Levantamentos geológicos: consistem em medidas de comprimento, posicionamento, orientação, espaçamento, abertura, e são baseadas em afloramentos, fotografias aéreas e amostras de núcleo (borehole core). Dados são obtidos em escala de campo, apesar dos cores serem geralmente estudados em laboratório.

- Técnicas geofísicas: consistem em métodos sísmicos e elétricos, radar de penetração no solo (GPR), técnicas de imagem de boreholes e tomografia hidráulica. Estas técnicas complementam as informações sobre localização, orientação, espaçamento e abertura, e são feitos em escala de campo e de laboratório.

- Testes hidráulicos em poços: testes hidráulicos com packers podem fornecer informações sobre condutividades hidráulicas e taxas de escoamento em fraturas únicas ou em formações maciças. Testes de variação de pressão, injeção de ar e níveis de água também podem ser usados. Traçadores podem ser usados analisando o comportamento do traçador para caracterizar o escoamento em um conjunto de fraturas.

É interessante notar que as próprias características de um sistema fraturado dificultam um mapeamento completo e detalhado in situ e, portanto, todas as medidas dependem de fatores subjetivos ou extrapolações. Medidas diretas de fraturas, a menos de dados limitados a partir de informações geológicas, não são possíveis com os métodos descritos acima. O que é medido são as respostas devido à presença de fraturas. Desta forma, para o estudo de fraturas, descrições estatísticas, interpolação e extrapolação são comumente usados.

A geometria de uma fratura é um fator essencial para os processos que nela ocorrem (32). O modelo conceitual mais comum é o de que a fratura é formada por placas paralelas suaves e planas $(26,32)^{1}$. Entretanto, a influência da rugosidade das paredes das fraturas no escoamento e transporte vem sendo estudado, mostrando que as paredes são geralmente irregulares, possuindo propriedades fractais com auto-afinidade (32). A fractalidade da rugosidade parece não depender da mineralogia, direção da fratura, ou do mecanismo de formação da mesma. Tais rugosidades são medidas em escala de laboratório. O entendimento do motivo da fractalidade com auto-afinidade e as diversas escalas de rugosidade são um problema em aberto, apesar de avanços recentes na literatura $(61,62)$.

Por abertura entende-se a separação entre as duas paredes que formam uma fratura em sua

\footnotetext{
${ }^{1}$ ver seções 3.2 e 3.5 .1
} 
dimensão de maior comprimento. A definição de abertura torna-se mais complexa ao levar-se em conta a rugosidade. A abertura, geralmente, é considerada como um parâmetro de entrada na modelagem, e pode ser observada nos afloramentos e inferida para a fratura. Processos de laboratório comumente "abrem" a amostra, não permitindo uma medida da mesma com a abertura preservada. Desta forma, a definição de abertura acaba tornando-se dependente do problema estudado (32), apesar de técnicas de imagem em laboratório usando ressonância magnética nuclear permitirem a inferência da abertura usando métodos não destrutivos (63).

Comprimento é a maior dimensão longitudinal da fratura. Orientação é o ângulo que a maior dimensão da fratura faz com relação ao norte magnético. Ângulo de mergulho é o ângulo que a fratura faz com a horizontal. Estes parâmetros também são assumidos como de entrada em um modelo. São medidos em campo ou assumidos, de acordo com distribuições estatísticas que descrevam o comportamento geral do sistema.

Dentre as diversas distribuições estatísticas usadas para descrever os parâmetros geométricos (como distribuições lognormal e normal), estudos recentes de propriedades de escala têm indicado um ajuste melhor usando descrições baseadas em leis de potência e características fractais (58). Um dado interessante sobre os sistemas que mostram comportamento fractal é a ausência de uma escala de homogeneização, o que previne a definição de um volume elementar representativo (Representative Elementary Volume, REV (26)). O transporte em sistemas fraturados é particularmente sensível às propriedades de escala do mesmo. Bonnet et al. (58) apresentam uma revisão da literatura sobre propriedades de escala em sistemas fraturados, incluindo um apêndice sobre a terminologia usada pelas diferentes comunidades (física, engenharia, matemática, geologia) na descrição dos mesmos fenômenos.

\subsubsection{Interconexão entre Fraturas}

A interconexão entre as fraturas é outra propriedade determinante no escoamento e transporte. Uma região pode ser altamente fraturada mas pouco conectada, levando a pouco fluido ser transportado pelas mesmas. Exemplos da influência da interconexão pode ser observada em poços perfurados em duas regiões com fraturas e geograficamente semelhantes (64). Testes de produção dos poços nestas duas localidades mostraram que a produção em uma das localidades foi maior, indicando uma maior interconexão entre as fraturas, do que os poços na outra localidade, que apresentaram baixa produção. Em um sítio estudado (65), foi constatado que apenas $0.1 \%$ das fraturas contribuíram para o escoamento em escala regional. Domínios que podem ser altamente fraturados podem não ser altamente conectados (32).

Neste contexto, o uso da teoria de percolação pode ser uma ferramenta efetiva para caracterizar a conectividade do sistema fraturado (32). Percolação é um modelo estatístico onde se consideram vértices conectados, com probabilidade $p$ de estarem abertos e $1-p$ de estarem fechados a passagem de um fluido, por exemplo. A pergunta é se existe um valor de $p$ para o qual o sistema está fechado ou aberto, ou seja, se conduz ou não o fluido. Este valor é chamado de $p$ crítico (percolation threshold), $p_{c}$, onde para valores abaixo a probabilidade de percolação é zero e acima, é um (66). 
Clusters de percolação tem natureza estatisticamente fractal, que podem ser usados para modelar meios heterogêneos e fraturados, e exibem comportamento independente de escala, o que permite que a teoria de percolação possa ser usada igualmente na escala de poros, laboratório e campo. Leis de potência derivadas da teoria de percolação na forma $A \propto\left(N-N_{c}\right)^{-X}$ descrevem diversas propriedades geométricas, de escoamento e transporte, como a chance de um sistema de fraturas ser hidraulicamente conectado. $A$ é uma grandeza física ou geométrica que pode ser observada, $X$ é um expoente relacionado a $A, N_{c}$ é o número de fraturas no limite de percolação crítico, e $\mathrm{N}$ é o número total de fraturas (32). O limite de percolação pode ser definido, por exemplo, como a densidade de fraturas suficiente para a qual o escoamento ocorre em pelo menos uma parte da rede de fraturas, de um lado do domínio ao outro. Mas mesmo modelos de percolação encontram dificuldades para considerar corretamente as propriedades de interconexão de sistemas de fraturas naturais $(32,33)$. Neste trabalho não serão feitos estudos de percolação.

\subsubsection{Limites na Descrição de Sistemas de Fraturas}

Devido à incerteza na quantificação e medida das grandezas que descrevem a geometria e fatores de escala, a modelagem dos sistemas fraturados ainda é falha. O problema é agravado devido à obtenção de dados ser geralmente uni ou bidimensional (1D ou 2D), e as fraturas serem tridimensionais (3D). Muitas vezes dados obtidos de forma uni ou bidimensional devem ser extrapolados para estruturas tridimensionais. Esta extrapolação de dados para uma uma dimensionalidade mais alta, chamada de inversão de dados, geralmente gera um problema mal condicionado. A partir dos dados parciais disponíveis diversas realizações de sistemas de fraturas 3D são geradas, de forma estocástica, e são comparadas com os dados obtidos em 2D e 1D, até que a resposta seja "satisfatória". Mesmo nestes modelos, o problema da unicidade da inversão dos dados permanece, e uma quantidade de dados e análise muito maior deve ser feita mesmo para somente estabelecer os limites de inversão.

\subsection{Escoamento em Uma Fratura}

O modelo clássico para a descrição de uma fratura, o de placas paralelas lisas, é a base de diversos modelos. Para esta geometria, a solução de estado estacionário da equação de Navier-Stokes para um fluido incompressível e escoamento laminar leva à chamada lei cúbica, para a qual a taxa volumétrica de escoamento é proporcional ao cubo da abertura $a$ entre as placas:

$$
Q=-\rho g \frac{a^{3}}{12 \mu} \vec{\nabla} \phi
$$

na qual $Q$ é a taxa de escoamento volumétrica ou descarga, $a$ é a largura da fratura, $\mu$ é a viscosidade do fluido, e $\vec{\nabla} \phi$ é o gradiente de carga causador do escoamento. A dedução da lei cúbica a partir da eq. de Navier-Stokes está descrita na seção 3.5.1.

Para levar a rugosidade (ou tortuosidade) em conta, uma aproximação da equação de Navier- 
Stokes, chamada de equação de Reynolds, geralmente é usada. A equação é descrita como a relação entre o gradiente de pressão e a velocidade, com o fator de proporcionalidade sendo igual ao cubo da abertura da fratura, na forma

$$
\nabla \cdot\left(a^{3} \nabla p\right)=0
$$

com as condições de que o fluxo deve ser laminar, a inclinação das paredes não é grande, o escoamento viscoso é dominante em relação aos termos inerciais e a largura da lâmina de líquido é pequena em relação ao comprimento do canal (67). Nota-se que agora a abertura pode variar, mas deve ter uma variação suave.

A validade da equação de Reynolds para fraturas com paredes rugosas com características fractais permanece também como uma questão em aberto $(32,63,67)$, com indicações de que a equação de Reynolds superestima a velocidade do fluido conforme a rugosidade aumenta $(67,68)$. Além deste problema, a própria definição de abertura é complexa, devido à rugosidade das paredes. Dentre os diversos métodos para a determinação de abertura, o método de Mourzenko et al. (68) sugere uma esfera com centro na superfície central, estendendo até as paredes e as tocando, sendo o mais robusto dentre os existentes. A partir de estudos de Ressonância Magnética Nuclear, o escoamento de água dentro de uma fratura foi estudado, mostrando que o perfil parabólico esperado em um perfil de placas paralelas é assimétrico em uma fratura real (63), com diferenças de até $20 \%$ no perfil de velocidades entre a fratura real e a modelada pela lei cúbica. O artigo conclui que para as características de abertura da fratura, da ordem de milímetros, a lei cúbica local é válida para o escoamento, mas os fenômenos decorrentes da rugosidade terão efeitos significativos para o transporte (63). Apesar das restrições apresentadas, a lei cúbica será usada no restante deste trabalho.

Crandall et al (3) fizeram uma análise do escoamento em 4 fraturas com perfis diferentes, provenientes de dados experimentais. No artigo, os autores descreveram a geometria dos perfis da fratura, encontrando que a rugosidade obedece um padrão similar a uma estrutura fractal browniana. Os autores então simularam o escoamento usando a equação de Navier-Stokes, testando as condições de contorno (assumindo que não há aderência na borda ou com deslizamento), e incluindo o meio poroso adjacente, com uma variação na permeabilidade do mesmo de 0,2 a 2000 millidarcies $\left(0,9869233\left(\mu \mathrm{m}^{2}\right)\right.$. Variando a condição de contorno nas paredes da fratura nas simulações, uma variação de $10 \%$ em média na velocidade de escoamento foi encontrada.

Wendland e Himmelsbach (60) fez estudos experimentais em um bloco de arenito com uma fratura única e experimentos numéricos variando diversos parâmetros nas fraturas. Nos experimentos numéricos a fratura foi modelada com elementos finitos, usando a descrição de placas paralelas e a lei de Darcy. A difusão na matriz porosa foi considerada como importante no mecanismo de transporte, atuando como um fator retardante. O estudo considerou primeiro um modelo tradicional de abertura constante, concluindo que este modelo não reproduz corretamente as curvas de passagem breakthrough. A causa provável para tal comportamento foi considerada como a influência da rugosidade das paredes e a dificuldade de determinar a abertura de forma experimental. Uma descrição estocástica auto-correlacionada das aberturas da fratura foi usada, e diversas simulações 
foram realizadas variando a correlação, o erro médio e a abertura média. A autocorrelação foi o parâmetro mais importante para reproduzir o comportamento da curva de passagem breakthrough e o modelo de placas paralelas foi adequado para a simulação de escoamento e transporte se o desvio médio da abertura é pequeno em relação à abertura média.

Oron et al. (69) argumentam que a lei cúbica pode ser aplicada localmente, se o segmento local tiver um comprimento suficiente comparado com a abertura, se a rugosidade local mantiver-se dentro de uma determinada faixa de valores, e os efeitos inerciais forem limitados, com número de Reynolds menor que um. Os autores também argumentam que a abertura deve ser calculada usando uma média pelo comprimento, e não ponto a ponto. Fraturas com auto-afinidade apresentam um acréscimo não linear na área de contato, e um decréscimo mais rápido que a lei cúbica na condutividade hidráulica com a diminuição da abertura. A rugosidade na parede da fratura também é um fator importante no fenômeno de channeling (68), descrito em (3.3.2). Outro fenômeno importante que ocorre é o desemparelhamento entre as duas paredes, ou seja, um deslocamento relativo entre a orientação das paredes. Um pequeno deslocamento, da ordem de 0,5 milímetro, pode mudar a condutividade hidráulica em até cinco ordens de magnitude, dependendo do comprimento (32).

A validade da lei cúbica para escoamento em uma fratura, em sua forma geral ou local, é uma questão em aberto, tanto pela validade do modelo quanto pela dificuldade de se obter dados (abertura). Modelos usando técnicas mais recentes, como a Hidrodinâmica de Partículas Suavizada (Smooth Particle Hydrodynamics, SPH), tentam mostrar em escala de fratura os efeitos das paredes e da estrutura da fratura no escoamento e no transporte (70).

\subsection{Escoamento em uma Rede de Fraturas}

Diversos modelos foram criados para tentar abarcar a grande variedade de parâmetros possíveis para uma rede de fraturas. Duas classes de modelos mais gerais são comuns: os modelos de fraturas discretas e os modelos contínuos, apesar de haver uma sobreposição entre as duas classes. A caracterização parcial das fraturas do domínio, a única que em muitos casos é possível, leva a uma descrição parcial de fraturas discretas e propriedades volumétricas do material poroso para as outras regiões. Por exemplo, a presença de fraturas em uma região pode impedir a escolha de uma escala de homogeneização, e portanto impossibilita a definição de um volume elementar representativo (REV), e desta forma limita o uso de modelos contínuos nesta região (71). Uma saída é o uso de modelos híbridos, pelos quais fraturas podem ser descritas por elementos discretos e por regiões contínuas, dependendo da quantidade de dados conhecidos e das características das mesmas.

Tanto os modelos contínuos quanto os discretos podem ser divididos em modelos determinísticos, para os quais um parâmetro, como a condutividade hidráulica, caracteriza as propriedades de escoamento e fluxo, e modelos estocásticos, para os quais os parâmetros e o próprio domínio são descritos por distribuições de probabilidade e campos aleatórios. Um exemplo consiste em uma simulação de Monte Carlo baseada em várias realizações diferentes do sistema de fraturas. Modelos estocásticos, por serem mais flexíveis e possibilitar uma faixa maior de comportamentos, 
são considerados serem de utilidade prática maior que os modelos determinísticos (32).

\subsubsection{Modelos Contínuos para um Sistema de Fraturas}

De forma geral, a abordagem contínua pode ser usada em uma rede de fraturas densa e altamente conectada. Para estes casos um REV pode ser definido. Já no caso onde a escala que o único REV que pode ser definido é a escala do domínio de interesse, o uso do REV não é indicado (26). Os modelos contínuos consistem em um contínuo, dois contínuos ou vários contínuos interagentes. O modelo de um contínuo é aplicado quando a rede de fraturas é altamente conectada e densa, e o meio poroso pode ser ignorado. Em um modelo de dois contínuos, o meio poroso e as fraturas são tratados como contínuos independentemente, sendo necessária uma relação de conservação de massa entre ambos. No caso de vários contínuos, conjuntos de fraturas com propriedades distintas são descritos por contínuos distintos, assim como o meio poroso. Existe uma diferença entre os modelos de dupla porosidade e dupla condutividade hidráulica: os primeiros assumem que o meio poroso é somente um local de armazenamento e liberação de fluido e solutos, enquanto no segundo o meio poroso assume papel ativo no escoamento e transporte. Na maioria dos casos não é possível assumir um modelo contínuo para sistemas fraturados, por existir uma dependência direcional no escoamento e a influência de diversas escalas para as propriedades das fraturas para o transporte (33).

\subsubsection{Modelos Discretos para um Sistema de Fraturas}

Modelos discretos permitem a descrição de fenômenos que modelos contínuos não conseguem descrever, por serem capazes de incorporar explicitamente propriedades de fraturas individuais $(71,72)$. Modelos discretos consideram cada fratura de forma independente, descrevendo suas propriedades geométricas e a interconexão entre elas. As fraturas modeladas de forma discreta podem ser representadas por elementos uni, bi e tridimensionais. Parâmetros como comprimento, abertura e rugosidade são atribuídos a cada fratura (72).

Tais modelos, apesar de terem limitações de ordem computacional em problemas para grandes domínios ou muitas fraturas, são bastante difundidos e usados em problemas práticos. Entretanto, sua aplicação é limitada pela maior necessidade de dados para seu uso e calibração, apesar de alguns experimentos terem sido projetados exclusivamente para um modelo desta natureza $(71,72)$. Modelos discretos apresentam melhores resultados quando usados de forma estocástica, com várias realizações diferentes do mesmo sistema de fraturas, além de permitirem a inclusão de propriedades dependentes de escala. Um exemplo é o trabalho de Hitchmough et al. (73), no qual um estudo experimental de um sistema de fraturas foi realizado na Inglaterra e um modelo de uma rede de fraturas usando uma distribuição estocástica de aberturas, direção e densidade, foi usado. Os dados de condutividade hidráulica foram estimados de forma estatística a partir de dados experimentais em escala de laboratório, e depois adaptados (upscaled) para a escala de campo. Os autores afirmaram que um cubo de $35 \mathrm{~m}$ é suficiente para a definição de um REV para o sistema, a partir de várias simulações da rede numérica de fraturas. O experimento foi realizando variando o tamanho do cubo 
simulado, e comparando com um volume de 35 m extraído da área e analisado em laboratório.

As redes discretas de fraturas são geralmente geradas usando distribuições estatísticas baseadas nas propriedades encontradas no campo, como orientação, distribuição de comprimento, posição, densidade, abertura, correlação ou anisotropia. A rede de fraturas então é gerada com o propósito de ser similar estatisticamente à rede observada $(32,33,71)$. Um dos problemas para o uso de rede discretas é a obtenção de dados. Dados experimentais podem não ser suficientes para determinar alguns destes parâmetros, para qual porcentagem das fraturas realmente conduzem fluidos, apesar da rede parecer ser bem conectada $(33,71)$. O esforço computacional necessário para a simulação usando uma rede discreta pode ser grande, e simplificações como o uso de modelos de placas paralelas podem ser usados para reduzir tal esforço, apesar das diversas evidências de que tal abordagem não é suficiente para reproduzir os resultados experimentais em escala de campo ou de laboratório $(33,60,71,74,75)$.

Outra forma de geração da rede é o uso dos parâmetros para a criação de uma rede aleatória ou correlacionada, formada por segmentos de diferentes condutividades hidráulicas, que represente, ao menos empiricamente, o comportamento do sistema de fraturas (76). Os diversos modelos existentes consideram certos aspectos da influência da conectividade e da distribuição de condutividade hidráulica do sistema de fraturas, tanto no plano de fraturas quanto na escala da rede de fraturas total. Outros modelos substituem as heterogeneidades e as fraturas por sistemas 1D estatisticamente equivalentes, de abertura variável (33).

Após a geração da rede discreta de fraturas, o escoamento nas mesmas deve ser determinado. Os modelos de escoamento são dependentes da forma de geração da rede, mas geralmente são divididos em duas classes: uma considera que o escoamento ocorre somente nas fraturas, e o outro que há escoamento no meio poroso também. No primeiro caso, como o fluxo é contido somente nas fraturas, o escoamento em si é simplificado, e geralmente dado por uma relação linear entre a taxa de escoamento e o gradiente de carga, com a condutividade hidráulica da fratura sendo a constante de proporcionalidade (escoamento obedecendo a lei de Poiseville). No modelo de Cacas et al. (71), por exemplo, a carga hidráulica é computada escrevendo a equação de balanço de massa para cada nó da rede de fraturas, formando um sistema linear com um número de variáveis igual ao número de conexões na rede (71). Esse modelo usa condutividades hidráulicas calibradas por testes de injeção hidráulica, não considerando a condutividade como um parâmetro externo independente.

\section{Channeling}

Em uma rede de fraturas com grande variação na condutividade hidráulica ou abertura, próxima ao limite de percolação, caminhos preferenciais de escoamento podem ser formados (32, 33, 68, 71). Este fenômeno é chamado de channeling, e tal fenômeno pode ser o mecanismo predominante no escoamento e transporte no meio fraturado, dependendo do grau de conectividade do mesmo e da rugosidade. Mourzenko et al. (68) demonstram em simulações numéricas que ao diminuir a abertura média das fraturas, o escoamento torna-se mais heterogêneo e passa a ocorrer em caminhos preferenciais. Mesmo em uma rede de fraturas bem conectadas caminhos preferenciais podem 
surgir, mesmo estando no limite de percolação, se a variação de condutividades hidráulicas for suficientemente grande.

Esses modelos, além de todas as incertezas, ainda não são bem integrados com testes de campo, como testes de bombeamento. O próprio planejamento de testes de campo é prejudicado pela incerteza na determinação das propriedades do sistema fraturado.

\subsection{Transporte}

O transporte de solutos está intrinsecamente ligado com as características de escoamento em fraturas, onde fenômenos como sorção, dissolução e precipitação podem surgir e necessitam de consideração na evolução do escoamento e do transporte.

Uma das abordagems para modelagem de transporte é o uso de uma equação de advecçãodispersão (ADE), com o meio fraturado sendo tratado como um meio poroso equivalente. As curvas de concentração para meios fraturados, tanto no laboratório quanto no campo, têm como característica instantes de chegada inicial curtos, um ou mais picos na concentração, e longas caudas (tails) $(32,77,78)$. Simulações baseadas em planos com aberturas geradas estatisticamente mostram o mesmo comportamento. Outra abordagem é o uso de modelos 2D, onde ocorre o aparecimento de fenômenos como channeling e caminhos preferenciais.

Os experimentos realizados nas últimas duas décadas mostram que, mesmo em domínios fraturados geologicamente muito estudados e muito bem caracterizados, os dados obtidos são insuficientes para permitir uma predição confiável e quantitativa de transporte de solutos. O grau de conectividade, algo dificilmente caracterizado in situ, leva ao aparecimento inesperado do traçador em poços de monitoramento, e variações grandes nos tempos de chegada em outros poços. Experimentos com traçadores em sistemas fraturados em escala de campo também apresentam tempos de chegada iniciais rápidos, mais de um pico de concentração nas curvas de passagem (breakthrough), longas caudas e dependência na escala $(77,78)$. Tal comportamento pode ser explicado pela rápida propagação do soluto por canais abertos (channeling), sendo boa parte do soluto retardada pela difusão no meio poroso $(60,77)$.

A permeabilidade no sistema de fraturas é geralmente bem maior que a permeabilidade do meio poroso, o que leva a velocidade do fluido nas fraturas a ser bem maior, transportando o soluto mais rapidamente (79). Entretanto, efeitos de retardamento, difusão do soluto e reações químicas podem ocorrer nos blocos porosos. Desta forma, efeitos de interação entre fratura e meio poroso pode ser muito mais importante para o transporte do que para o fluxo.

Métodos estocásticos permitem variação na descrição do transporte dependente de escala. Berkowitz e Schen (74) argumentam que mesmo para casos com heterogeneidade não muito complexa o transporte de solutos não obedece a lei de Fick, e a equação de difusão-dispersão não pode ser usada, levando ao que é chamado de dispersão anômala ${ }^{2}$. Os autores também argumentam

\footnotetext{
${ }^{2}$ Dispersão anômala é definida quando os coeficientes de transporte têm dependência temporal ou espacial.
} 
que a variação de aberturas em uma rede de fraturas não é suficiente para causar a dispersão anômala: a variação na interconexão na rede fraturas em função de seu comprimento e conexões também é crucial. Se, mesmo em uma rede de fraturas com variação na interconexão, há uma média entre o número de interconexões e os locais de encontro em cada caminho, o coeficiente de dispersão tende a uma constante. Uma das causas apontadas para este comportamento são as regiões sem conectividade, onde há uma variação local na velocidade de escoamento. Nestas regiões o soluto é aprisionado por um determinado tempo, sendo liberado posteriormente. Devido à dificuldade de obter dados experimentais diretos das fraturas, modelos estocásticos gerados a partir de fraturas aflorantes são gerados para caracterizar as mesmas $(59,75)$. Os autores apresentam um método para melhor abordar o problema do transporte não-Gaussiano (anômalo), que pode ser dependente de características sutis do sistema de fraturas. Eles propõem um método chamado Continous Time Random Walk (CTRW). Este método permite preservar a correlação espacial e temporal entre as partículas conforme elas são transportadas por advecção e difusão no fluido. No artigo (59), Berkowitz e Scher descrevem em maior detalhe o método, e estabelecem as condições para a simulação. $\mathrm{O}$ escoamento dentro das fraturas é do tipo plug flow ${ }^{3}$ e a mistura (mixing) é completa nas interseções das fraturas ${ }^{4}$.

Hakami et al. (80) apresentam um diagrama para o contexto experimental no estudo de fluxo de água subterrânea em rochas fraturadas. Em meios com baixa condutividade hidráulica, o fluxo ocorre predominantemente nas redes de fraturas, e o escoamento nas fraturas é governado principalmente pelas características de escoamento de fraturas individuais. A definição de abertura hidráulica provém de experimentos nos quais a variação no escoamento devido à variações de pressão são estudadas. Assumindo que as fraturas podem ser descritas como placas planas paralelas, a abertura das fraturas que representa o experimento compõe a abertura hidráulica, uma abertura efetiva. Nos testes experimentais feitos, para uma pressão lateral na amostra de 0,45 MPa, a abertura média foi de $360 \mu \mathrm{m}$ com um desvio padrão de $150 \mu \mathrm{m}$ e a abertura hidráulica é de $250 \mu \mathrm{m}$. A diferença entre a abertura média e a abertura hidráulica é explicada como sendo causada pela variação da abertura ao longo da fratura, que força o fluxo a ser tortuoso. Hakami et al. também enfatizaram que os resultados obtidos são específicos para a fratura estudada, servindo apenas como um exemplo para casos reais. A correlação espacial da fratura também foi medido, girando em torno de 5-20 milímetros. Uma simulação usando um modelo com aberturas variáveis foi feita, e a relação entre o fluxo medido e o fluxo modelado foi de 2.4 vezes maior na simulação, indicando que as propriedades das fraturas não foram bem descritas. Os resultados de (80) são similares aos encontrados por (60).

Ando et al. (81) apresentaram uma comparação entre dados experimentais e uma simulação de transporte usando uma rede discreta estocástica, em um sítio chamado Fanay-Augères, com os resultados para redes discretas de (71). Ando et al. concluem que sistemas abertos, especialmente complexos como redes de fraturas, não podem ser descritos unicamente com base em dados esparsos. Entretanto, não precisam ser descritos em detalhe para capturar o comportamento principal do

\footnotetext{
${ }^{3}$ ou seja, a velocidade de escoamento é considerada constante em qualquer seção do tubo, perpendicular ao eixo do tubo.

${ }^{4}$ Partículas que saem de uma interseção entre duas fraturas tem distribuição aleatória, dependendo somente da velocidade do fluido em cada fratura.
} 
sistema.

\subsection{Modelos e Abordagens Numéricas para Sistemas Fra- turados}

\subsubsection{Modelo de Placas Paralelas - a Lei Cúbica}

A equação de Navier-Stokes descreve o escoamento em escala microscópica de um fluido. A partir da equação tridimensional microscópica, quando os termos inerciais e os termos de atrito são desprezados, a lei de Navier-Stokes é reduzida à lei de Darcy. Um modelo para fraturas que vem sendo usado na literatura (26) é o modelo de placas paralelas, que assume que as paredes da fratura são duas placas paralelas e lisas. A partir deste modelo um perfil de velocidade é encontrado e o escoamento na rede, calculado. Esta seção acompanha o desenvolvimento feito por (26) e (82).

Algumas definições são introduzidas de forma a permitir a modelagem das fraturas. As paredes da fratura são descritas por duas superfícies, $F_{1}(x, y, z)=y_{1}-f_{1}(x, y)$ e $F_{2}(x, y, z)=y_{2}-f_{2}(x, y)$, onde $y$ é a coordenada do eixo da fratura até a parede. $O$ eixo da fratura é definido como o centroide de esferas com suas superfícies inscritas entre as paredes. Estes centróides podem ser aproximados por um plano, o plano do eixo da fratura. O vetor normal unitário da superfície, $\vec{n}$, é definido como

$$
\vec{n}=\frac{\vec{\nabla} F_{i}}{\left|\vec{\nabla} F_{i}\right|}
$$

A abertura, como já visto, é a distância entre as paredes que delimitam a fratura:

$$
a(x, y)=f_{2}(x, y)-f_{1}(x, y)=y_{2}-y_{1} \text {. }
$$

A equação tridimensional de balanço de massa e momento para um fluido incompressível na fratura é dada pelas equações de Navier-Stokes:

$$
\rho \frac{\partial \vec{v}}{\partial t}+\rho \vec{\nabla} \cdot(\vec{v} \vec{v})+\vec{\nabla} p-\rho \vec{g}-\mu \nabla^{2} \vec{v}=0
$$

$\rho$ é a densidade absoluta do fluido, $\mu$ é a viscosidade dinâmica, $p$ é pressão, $\vec{v}$ é a velocidade do fluido em média da massa, e $z$ a coordenada vertical. A operação $\vec{v} \vec{v}$ é uma díade, e o gradiente de uma díade resulta em um tensor. Substituindo-se a pressão pela carga hidráulica, a equação de balanço de momento e massa (também chamada de equação governante) fica igual a

$$
\rho \frac{\partial \vec{v}}{\partial t}+\rho \vec{\nabla} \cdot(\vec{v} \vec{v})+\rho g \vec{\nabla} \phi-\mu \nabla^{2} \vec{v}=0
$$

Diversas simplificações precisam ser feitas para que a equação governante possa ser resolvida. As simplificações são

- o efeito da advecção é muito maior que o da dispersão, 
- a carga $\phi$ é igual nas duas superfícies da fratura: $\phi_{f_{1}}=\phi_{f_{2}}$,

- o escoamento é estacionário,

- o escoamento é unidirecional, na direção $x$,

- o escoamento é unidimensional, somente na direção de fluxo $x$,

- as paredes das fraturas são planas: $f_{1}=f_{2}=$ cte.

- o escoamento é viscoso e lento

As simplificações listadas em (3.5.1) permitem desprezar os termos inerciais na equação de NavierStokes (3.6). A equação resultante das simplificações aplicadas à (3.6) é

$$
\begin{array}{r}
\rho g \frac{\partial \phi}{\partial x}=\mu \frac{\partial^{2} v_{x}}{\partial y^{2}} \\
\frac{\partial \phi}{\partial y}=0
\end{array}
$$

Assumindo que não há escorregamento nas paredes $\left(v_{x}=0\right)$, e não há fluxo através delas, a distribuição de velocidades se torna parabólica:

$$
\begin{array}{r}
v_{1}(y)=\frac{6 \tilde{v_{x}}}{a^{2}}\left(\frac{1}{2} a+y\right)\left(\frac{1}{2} a-y\right) \\
\frac{1}{2} a \leq y \leq \frac{1}{2} a
\end{array}
$$

Com este perfil de velocidade, uma relação entre a velocidade do fluido na fratura e o gradiente de carga médio pode ser estabelecida, formando a lei de Darcy:

$$
\tilde{v_{x}}=-\frac{\rho a g}{\mu} \frac{a^{2}}{12} \frac{d \tilde{\phi}}{d x}
$$

Usando a definição de permeabilidade de (2.33),

$$
\tilde{v_{1}}=-K_{f r} \frac{d \tilde{\phi}}{d x}
$$

Considerando a relação entre a condutividade hidráulica e a permeabilidade, obtemos a relação

$$
K_{f r}=\frac{\rho g}{\mu} k
$$

tal que a permeabilidade de uma fratura é dada por

$$
k=\frac{a^{2}}{12}
$$

Considerando que o escoamento é saturado (ou seja, confinado dentro da fratura), a descarga é obtida como

$$
Q_{f r}=b \tilde{v}=-\frac{\rho g}{\mu} \frac{a^{3}}{12} \vec{\nabla} \tilde{\phi}=-T_{f r} \vec{\nabla} \tilde{\phi}
$$


onde a transmissividade da fratura $T_{f r}$ é igual a

$$
T_{f r}=\frac{\rho g}{\mu} \frac{b^{3}}{12}
$$

de onde vem a definição da "lei cúbica" para placas paralelas.

\subsubsection{Aproximação Linear para a Abertura}

Uma alternativa ao uso da lei cúbica para o escoamento de fraturas, mas baseada no mesmo desenvolvimento, considera que o interior da fratura não é vazio, mas sim preenchido por um material com condutividade hidráulica muito mais alta que o meio poroso. As equações de movimento e as aproximações dadas em (3.5.1) ainda são válidas, mas com a inclusão do material dentro da fratura, a equação de descarga torna-se (4)

$$
Q_{f r}=-\frac{K^{*} b}{K} \frac{\partial \phi}{\partial x_{1}}
$$

Esta aproximação será usada na comparação entre o elemento para fratura e uma inomogeneidade poligonal similar à fratura. 


\section{$4 \quad$ O Método de Elementos Analíticos}

\subsection{Introdução}

No Método de Elementos Analíticos (MEA), originalmente desenvolvido por Otto Strack (1) em seu livro Groundwater Mechanics, baseado em seus artigos com Haitjema (83, 84), a influência de cada elemento hidrogeológico do aquífero é representada por uma equação para o potencial de descarga. Estas expressões satisfazem exatamente as equações de dinâmica e de balanço de massa. Estas soluções da equação governante, chamadas de elementos, contém coeficientes desconhecidos que devem ser calculados usando as condições de contorno $(1,85)$. Devido à linearidade da equação governante com relação ao potencial de cada elemento, a superposição das soluções é usada, gerando a solução final do problema. Neste capítulo serão mostradas as base para o desenvolvimento do método de solução do MEA, usado para o modelo da fratura.

\subsection{O Método de Elementos Analíticos}

O Método de Elementos Analíticos foi desenvolvido para simular o escoamento de água subterrânea, e sua intenção original era simular domínios de escala regional (38). O método usa soluções da equação governante do escoamento em estado estacionário com rotacional e divergente nulos $(1,38)$, geralmente satisfazendo as condições de contorno internas de forma numérica no domínio (39). Cada elemento hidrogeológico é representado por uma solução da equação governante, chamado de elemento analítico, e têm sua condição de contorno determinada pelo fenômeno que representa. Como a equação de Laplace/Poisson (2.28) é linear para o potencial de descarga, as soluções para os aspectos hidrogeológicos podem ser superpostas e os graus de liberdade das soluções são determinados a partir das condições de contorno estabelecidas.

O método aproveita-se da teoria do potencial (86), já que para escoamentos irrotacionais o vetor descarga (2.4.3) é proporcional ao gradiente do potencial de descarga. Outra característica marcante do MEA é que ele não requer que o domínio seja limitado por fronteiras, sendo necessária apenas a condição de contorno no infinito(40) e as condições internas em determinados pontos.

As teses (51) e (55) fazem uma revisão bastante extensa da literatura existente sobre o MEA. O método tem seu nome derivado da notação das funções analíticas, funções complexas com propriedades úteis considerando a equação de Laplace. A definição das funções analíticas será 
discutida neste capítulo; mais informações podem ser encontradas nas referências $(87,88)$.

Como visto na seção 2.4.3, o escoamento da água subterrânea nas condições definidas (incompressível, irrotacional, estacionário) permite a descrição do mesmo através do potencial de descarga:

$$
\Phi(x, y)=\left\{\begin{array}{l}
K H \phi(x, y)-\frac{1}{2} K H^{2}, \text { confinado } \\
\frac{1}{2} K \phi^{2}(x, y), \text { não-confinado }
\end{array}\right.
$$

estabelecendo a relação entre a carga $\phi$ e o potencial de descarga $\Phi$. A equação governante é a equação de Laplace para o potencial de descarga:

$$
\nabla^{2} \Phi(x, y)=0
$$

Ao se usar a aproximação de Dupuit, o problema se torna bidimensional. Neste caso, o uso de variáveis complexas torna-se atraente, devido às propriedades das funções analíticas complexas com relação à equação de Laplace (88). Com o uso do potencial complexo $\Omega(z)$, o potencial de descarga $\Phi$ forma a parte real de $\Omega(z)$, e as linhas de corrente (stream functions) $\Psi$ formam a parte imaginária de $\Omega(z)$. Linhas de corrente $\Psi$ são sempre perpendiculares às linhas de potencial de descarga $\Phi$, e indicam a trajetória do escoamento, sendo paralelas ao vetor velocidade (52).

Os números complexos podem ser definidos da forma usual, como um par ordenado $z=(x, y)$ dos números reais $x$ e $y$. O número imaginário puro $i=(0,1)$ pode ser usado na definição dos números complexos, de acordo com a sua propriedade de multiplicação $i * i=i^{2}=-1$ (87). Com estas definições, um número complexo será denotado neste trabalho como

$$
z=x+i y
$$

Usando números complexos na definição do potencial $\Omega(z)$, este toma a forma

$$
\Omega(z)=\Phi(x, y)+i \Psi(x, y)
$$

Usando esta definição, o vetor descarga é igual a

$$
\begin{aligned}
& Q_{x}(z)=-\frac{\partial \Omega(z)}{\partial x} \\
& Q_{y}(z)=-\frac{\partial \Omega(z)}{\partial y}
\end{aligned}
$$

e a equação de Laplace para o potencial complexo é igual a

$$
\nabla^{2} \Omega(z)=0
$$

O uso da equação de Laplace para variáveis complexas abre a possibilidade do uso do Cálculo de Wirtinger $(45,89)$. O cálculo de Wirtinger é baseado em uma transformação de variáveis e permite a integração direta da equação de Laplace no sistema de coordenadas de Wirtinger. O cálculo de 
Wirtinger permite trabalhar com funções complexas que não são analíticas, e com campos com rotacional e divergente diferentes de zero. É possível encontrar todos os resultados obtidos com variáveis complexas de forma tradicional com o cálculo de Wirtinger e extender estes resultados para outros casos, como escoamentos onde o rotacional é diferente de zero. Como as condições de escoamento deste trabalho assumem rotacional e divergente nulos, não há necessidade de empregar o uso do cálculo de Wirtinger, e o mesmo não será usado. Mais detalhes podem ser encontrados em (48).

Para dar uma idéia de como o MEA funciona, as figuras (4.1) a (4.4) demonstram um exemplo. A figura (4.1) mostra uma fotografia aérea da Bacia Piloto do Ribeirão da Onça (90). A figura (4.2) demonstra a discretização de poços, rio e lago por elementos analíticos - elemento analítico para poços, $\Omega_{w}$, line sinks (48) para o rio, $\Omega_{l s}$, e line sinks com carga constante para o lago, $\Omega_{l s^{\prime}}$. A próxima etapa na modelagem do AEM pode ser a inclusão de uma região com condutividade hidráulica diferente da do meio, representada por um polígono formado por line doublets, com o potencial complexo $\Omega_{l d}$. A figura (4.3) mostra esta inclusão. A figura (4.4) mostra apenas os elementos e seus potenciais complexos, feita a discretização dos elementos hidrogeológicos por elementos analíticos.

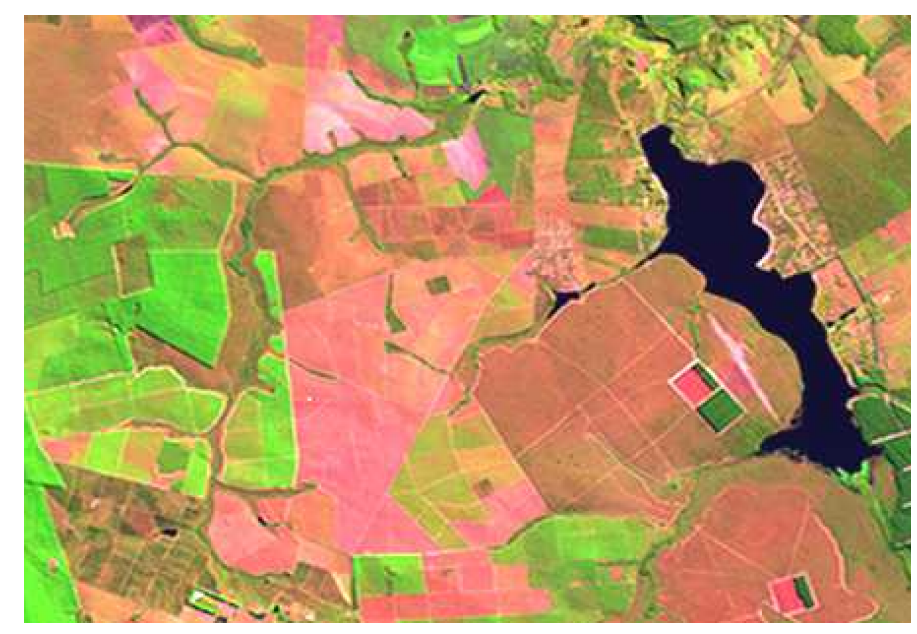

Figura 4.1 - Fotografia Aérea da Bacia Piloto do Ribeirão da Onça.

\subsubsection{Superposição}

Como a equação de Laplace (2.28) para o potencial é linear, pode-se usar o princípio da superposição. O princípio da superposição afirma que a resposta total causada por mais de uma fonte é igual à soma da resposta de cada fonte individualmente (91). As incógnitas do problema são encontradas usando as condições de contorno para todos os elementos. O comportamento final do escoamento em um determinado ponto é dado pela soma de todas as soluções representativas de elementos hidrogeológicos, mais uma constante dada por um valor prescrito de carga hidráulica em um ponto distante do domínio de interesse no aquífero. 


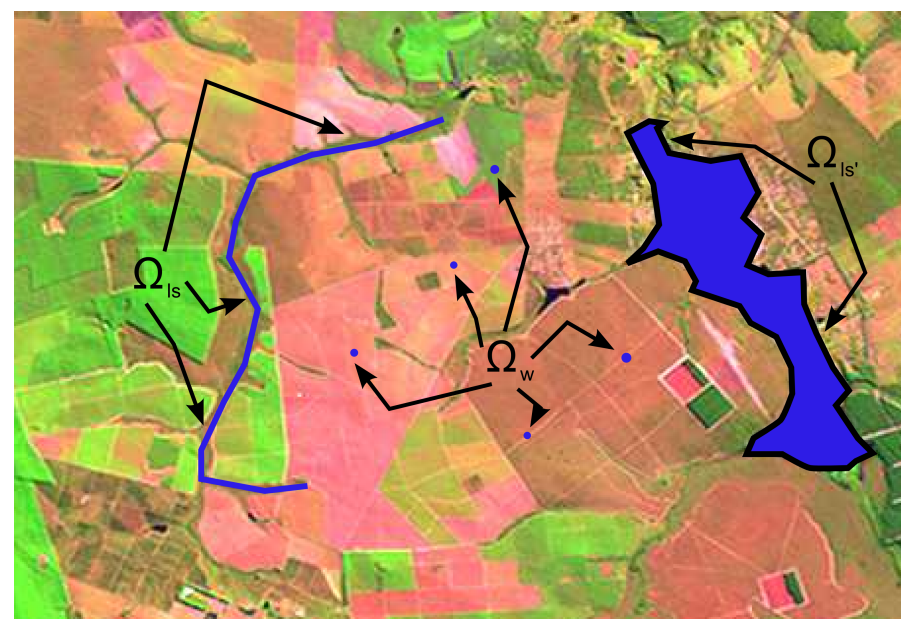

Figura 4.2 - Fotografia Aérea da Bacia Piloto do Ribeirão da Onça, com discretização dos elementos hidrogeológicos para rio, poço e lago.

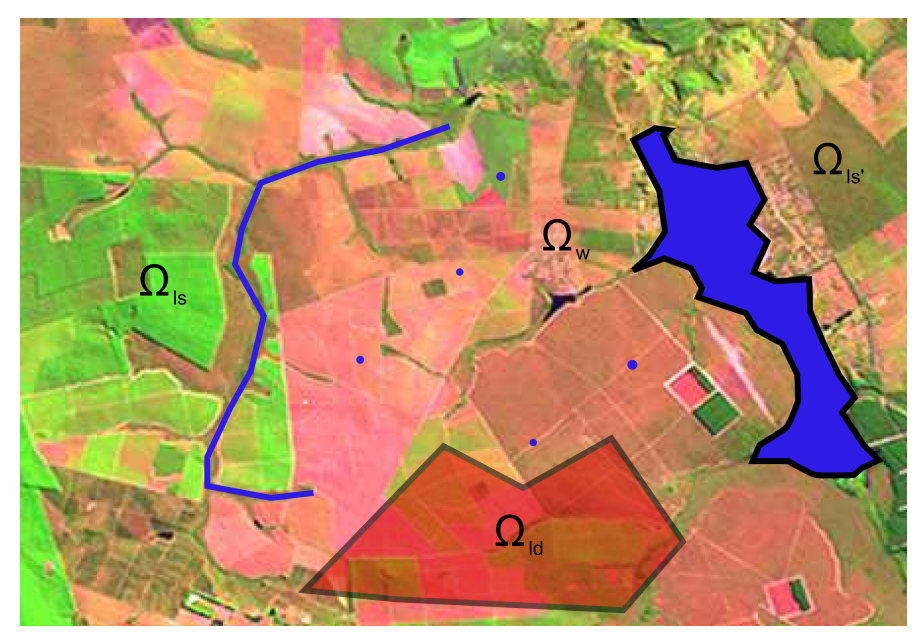

Figura 4.3 - Inclusão de uma inomogeneidade na condutividade hidráulica por um polígono formado por line doublets.

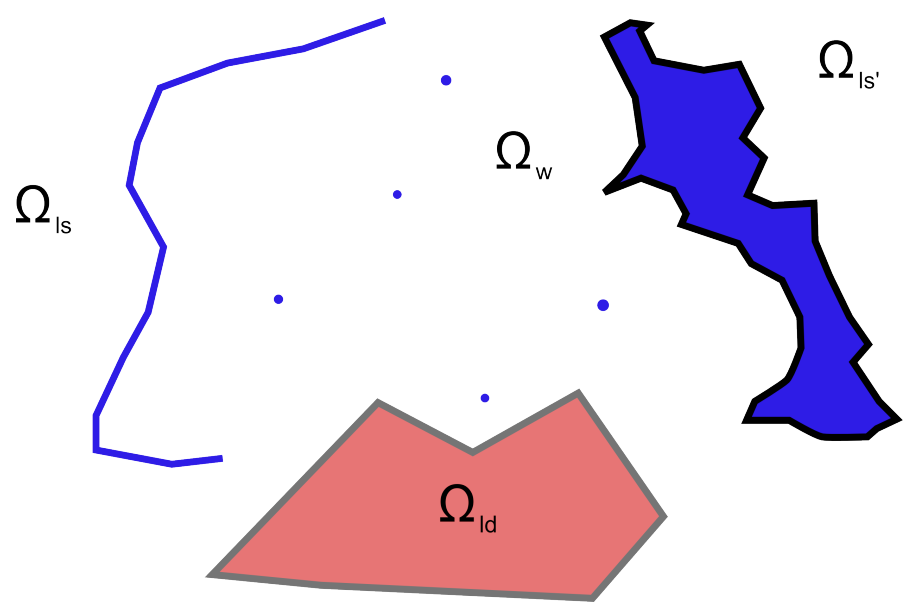

Figura 4.4 - Representação somente dos elementos analíticos usados para representar o domínio a ser estudado.

\subsection{Satisfazendo as Condições de Contorno}

Cada elemento, solução da equação governante, tem graus de liberdade a serem determinados, para que satisfaçam as condições de contorno de acordo com o tipo de elemento e o problema em 
questão. A forma como estas condições são satisfeitas é um dos problemas centrais do MEA. Os métodos mais utilizados na literatura estão descritos abaixo.

\subsubsection{Método de Colocação}

Uma das formas de satisfazer as condições de contorno sobre os elementos é calcular o valor exato da condição em pontos determinados ao longo do elemento. O número de pontos é igual ao número de coeficientes desconhecidos do elemento $(1,85)$. Apesar de ser de relativa simplicidade na implementação, o método de colocação apresenta como problema o erro entre os pontos onde a condição foi satisfeita. O erro entre os pontos aumenta ao se aumentar os graus de liberdade para um mesmo elemento, dependendo do caso a ser estudado $(37,85)$. Para cada ponto de colocação no elemento, a influência de todos os elementos é calculada, formando-se uma matriz para a qual cada linha é composta pelo valor do potencial de cada elemento no ponto de controle a ser calculado.

\subsubsection{Método dos Mínimos Quadrados}

Janković $(85,92,93)$ propôs o método de mínimos quadrados $(94)$ com o objetivo de melhor satisfazer as condições de contorno no MEA, calculando as condições de contorno em mais pontos do que graus de liberdade de cada elemento. Foi usado um conceito de média sobre os pontos, ao invés de tentar satisfazer exatamente as condições de contorno. O nome que foi dado ao método foi de método de sobreespecificação (overspecification method).

De forma geral, o problema de mínimos quadrados é um problema de minimização. Como exemplo, uma função $f(x)$ pode ser expandida em uma série com coeficientes desconhecidos,

$$
f(x)=\sum_{n} a_{n} x^{n}
$$

Dado um conjunto de pontos $x_{i}$ para o qual o valor da função $f(x)$ é conhecida, é possível encontrar os coeficientes desconhecidos minimizando o erro quadrático:

$$
\sum_{i}\left(f\left(x_{i}\right)-\sum_{n} a_{n} x_{i}^{n}\right)^{2} \rightarrow \operatorname{MIN}\left(a_{0}, \ldots, a_{n}\right)
$$

Uma forma de minimizar esta equação é derivar em relação às variáveis desconhecidas, $a_{n}$, e igualar a zero:

$$
\frac{\partial}{\partial a_{j}} \sum_{i}\left(f\left(x_{i}\right)-\sum_{n} a_{n} x_{i}^{n}\right)^{2}=0
$$

o que é

$$
\sum_{i}\left(f\left(x_{i}\right)-\sum_{n} a_{n} x_{i}^{n}\right) x_{j}=0
$$

Caso as funções base da expansão (4.9) forem ortonormais, o produto das funções que ocorre em 
(4.12) possibilita o uso da ortogonalidade, o que simplifica o cálculo dos coeficientes desconhecidos $a_{n}$.

\section{Uso de Vínculos}

Ao se usar o método de mínimos quadrados, é possível incluir vínculos (constraints) para valores conhecidos em determinados pontos nos elementos. Uma forma de incluir tais vínculos é através de multiplicadores de Lagrange (94). Os trabalhos que introduzem o método de mínimos quadrados faz uso de vínculos para os elementos linha $(85,92,93)$, reportando melhorias com relação a sistemas para os quais vínculos não são usados. Entretanto, existem casos onde a inclusão de vínculos não beneficia a solução do problema ${ }^{1}$. Vínculos não serão inclusos no presente trabalho.

\subsection{Definições Matemáticas}

Esta seção trata das definições matemáticas básicas a serem usadas no desenvolvimento do MEA, incluindo alguns desenvolvimentos recentes. O objetivo desta seção é apresentar os conceitos básicos necessários para o desenvolvimento do trabalho. Por se tratar de problemas bidimensionais, como já mencionado, variáveis complexas serão usadas. Deve-se notar que devido à relação entre a variável complexa $z$ e as variáveis $x, y$, a notação para funções é feita de forma intercambiável:

$$
f(z)=f(x, y)
$$

\subsubsection{Transformações de Coordenadas}

Uma das características interessantes em se trabalhar com variáveis complexas é a possibilidade do uso de transformações conformes ${ }^{2}$. Uma transformação que será usada extensivamente é a transformação de uma linha no plano $z$ de comprimento $L$ e orientação $\alpha$ em relação ao eixo $x$ para uma linha no plano $Z$ de comprimento 2 , na direção do eixo $X$. O sistema de coordenadas $z$, chamado de sistema de coordenadas global, é definido como

$$
z=x+i y
$$

e o eixo de coordenadas do sistema de coordenadas local $Z$, transformado, é definido como

$$
Z=X+i Y
$$

A transformação é dada por

$$
Z=\frac{z-\frac{1}{2}\left(z_{1}+z_{2}\right)}{\frac{1}{2}\left(z_{2}-z_{1}\right)}
$$

\footnotetext{
${ }^{1}$ Philippe Le Grand, comunicação pessoal, 2011

${ }^{2}$ Transformações conformes são transformações de coordenadas (mapas) que preservam ângulo
} 
na qual $z_{1}$ e $z_{2}$ são os pontos inicial e final, respectivamente, da linha no plano $z$. A transformação inversa é dada por

$$
z=Z \frac{1}{2}\left(z_{2}-z_{1}\right)+\frac{1}{2}\left(z_{1}+z_{2}\right)
$$

Esta transformação é demonstrada na figura (4.5), onde uma linha de comprimento $L$ e ângulo $\alpha$ é convertida para o sistema de coordenadas $Z$. Outra transformação usada é a transformação de Joukowski (95). Dada uma variável complexa $z$ o mapa dado por $w$ tem a forma:

$$
Z=\frac{1}{2}\left(\chi+\chi^{-1}\right)
$$

Se $\chi$ se move em um círculo $|\chi|=r$, com $r>1$, centrado na origem, a transformação é igual a

$$
\begin{array}{r}
\chi=r e^{i \theta}=r \cos (\theta)+i r \sin (\theta) \\
\chi^{-1}=r^{-1} e^{i \theta}=r^{-1} \cos (\theta)-i r^{-1} \sin (\theta)
\end{array}
$$

e a variável $Z$ se torna

$$
Z=\frac{1}{2}\left(r+r^{-1}\right) \cos (\theta)+i \frac{1}{2}\left(r-r^{-1}\right) \sin (\theta)
$$

com

$$
\begin{aligned}
& a=\frac{1}{2}\left(r+r^{-1}\right) \\
& b=\frac{1}{2}\left(r-r^{-1}\right)
\end{aligned}
$$

Portanto $Z=X+i Y$ forma uma equação elíptica:

$$
\frac{X^{2}}{a^{2}}+\frac{Y^{2}}{b^{2}}=1
$$

com excentricidade igual a

$$
a e=\sqrt{a^{2}-b^{2}}=1
$$

com foco em \pm 1 . A figura (4.6) ilustra a transformação direta (4.18). A transformação inversa é dada por

$$
\chi=Z \pm \sqrt{Z^{2}-1}=Z \pm \sqrt{Z-1} \sqrt{Z+1}
$$

enfatizando a ramificação causado pela raiz quadrada.

Se o módulo de $\chi$ é igual a 1 e $r=1$, a elipse no plano $Z$ é colapsada no eixo $X$. O eixo é percorrido duas vezes quando $\chi$ se move sobre o círculo: $Z$ varia de -1 a +1 quando $\theta$ varia $-\pi$ a 0 , de 1 a -1 quando $w$ varia de 0 a $\pi$. 

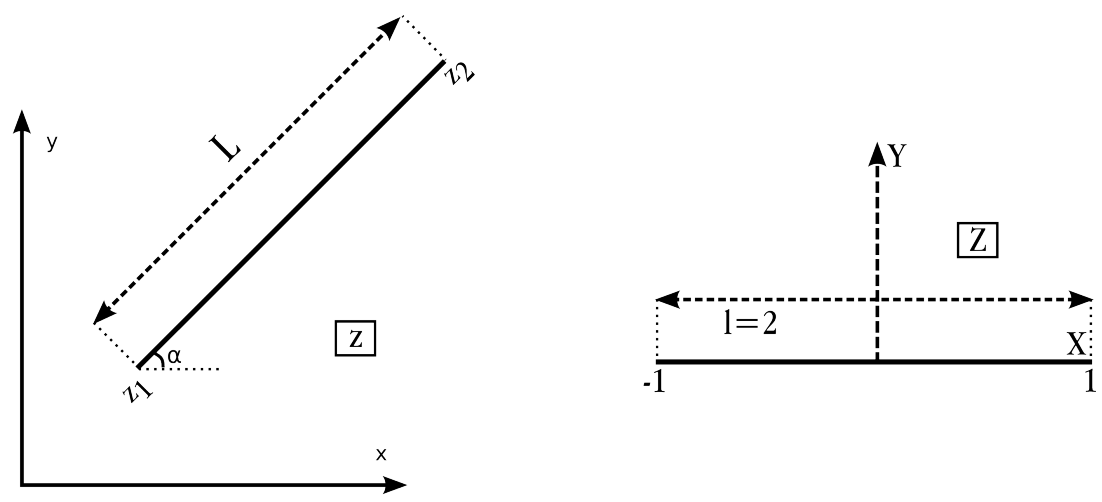

Figura 4.5 - Transformações de coordenadas lineares locais. A linha tem início em z1 e término em z2. A transformação para o plano Z leva para uma linha de início -1 e término 1.
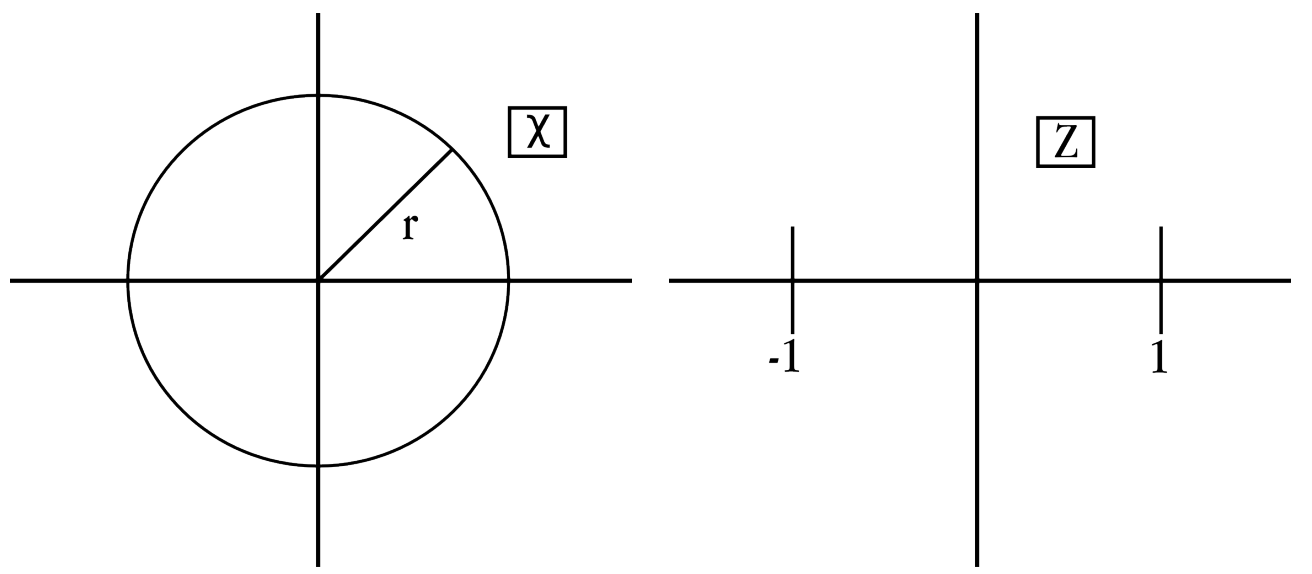

Figura 4.6 - Transformação de um círculo de raio r para uma elipse, ou para uma linha, caso r seja igual a 1.

A coordenada local $Z$ é transformada para o sistema de coordenadas local $\chi$. Ao transformar da linha no plano $\Re(Z)=X$ para o plano $\chi$, a variável $\chi$ é igual a $e^{i \theta}=\cos (\theta)+i \sin (\theta)$, por estar no círculo de raio unitário. Na transformação reversa, para que $\chi$ tenda para o infinito, quando $Z$ tende ao infinito, o sinal da transformação é escolhido positivo:

$$
\chi=Z+\sqrt{Z+1} \sqrt{Z-1}
$$

\subsubsection{Funções Holomórficas, Analíticas e Harmônicas}

Uma função é chamada de holomórfica se for diferenciável em cada ponto $z$ do domínio $D$. Uma função também é holomórfica em $z_{0}$ se for diferenciável em $z_{0}$ e em uma vizinhança $B$ (87). Como é diferenciável, a função holomórfica obedece as condições de Cauchy-Riemann $(87,88)$.

Uma função é chamada de analítica, se pode ser expandida em uma série de potências ao redor de um ponto $z_{0}(87,88)$ :

$$
f(z)=\sum_{n=0}^{\infty} a_{n}\left(z-z_{0}\right)^{n}
$$


Uma função holomórfica é sempre analítica. Se $f$ for holomórfica, a expansão em série coincide com a série de Taylor na vizinhança do ponto $z_{0}$ :

$$
f(z)=\sum_{n=0}^{\infty} \frac{f^{n}\left(z_{0}\right)}{n !}\left(z-z_{0}\right)^{n}=\sum_{n=0}^{\infty} a_{n}\left(z-z_{0}\right)^{n}
$$

Fazendo uma manipulação algébrica, é possível obter os coeficientes da expansão em série de potências a partir da expansão em série de Taylor:

$$
a_{n}=\frac{f^{n}\left(z_{0}\right)}{n !}
$$

Este resultado é útil ao se usar em conjunto o teorema de Cauchy-Goursat $(87,88)$ e a integral de Cauchy $(87,88)$, como descrito na seção (4.4.3). Se a função $f$ não satisfaz a condição de analiticidade em um ponto $z_{1}$ mas a satisfaz em uma vizinhança do ponto, $z_{1}$ é chamado de ponto singular, ou singularidade, da função $f$.

\subsubsection{Teorema de Cauchy-Goursat e a Fórmula Integral de Cauchy}

O teorema de Cauchy-Goursat afirma que dada uma função $f$, analítica em um domínio com contorno $C$, a integral de contorno em $C$ da função $f$ é igual a zero (87):

$$
\oint_{C} f(z) d z=0
$$

O inverso é verdadeiro: se uma função $f$ é contínua no interior do domínio de contorno $C$, no contorno $C$ e sua integral de contorno é igual a zero, a função $f$ é analítica, desde que o contorno seja geral.

A Fórmula Integral de Cauchy afirma que dada uma função $f$, analítica no interior do domínio com contorno $C$, no contorno $C$, tomado no sentido positivo, e dado um ponto $z_{0}$ qualquer interior a $C(87,88)$, o valor de $f$ é dado pela integral no domínio $C$ para o ponto $z_{0}$ :

$$
f\left(z_{0}\right)=\frac{1}{2 \pi i} \oint_{C} \frac{f(z)}{z-z_{0}} d z
$$

Uma outra forma de descrever o teorema é que se a função $f$ é analítica dentro e no contorno $C$, os valores de $f$ dentro de $C$ são determinados pelos valores de $f$ em $C$. Qualquer mudança no valor de $f$ em um ponto dentro de $C$ deve ser acompanhado com uma mudança em seu valor no contorno $C$ (87).

O teorema de Cauchy-Goursat e a Fórmula Integral de Cauchy serão usados para calcular os valores da condição de contorno para os diferentes elementos analíticos, como demonstrado abaixo. 


\subsubsection{Relação entre a Fórmula Integral de Cauchy e a Série de Taylor}

Usando os resultados das seções (4.4.2) e (4.4.3), pode ser estabelecida uma relação entre uma expansão em série de Taylor de uma função $f$ e a integral de Cauchy desta função em um contorno. Mudando a variável de integração, a primeira derivada da integral de Cauchy é igual a

$$
f^{\prime}\left(z_{0}\right)=\frac{1}{2 \pi i} \oint_{C} \frac{f(s)}{\left(s-z_{0}\right)^{2}} d s
$$

com a segunda derivada igual a

$$
f^{\prime \prime}\left(z_{0}\right)=\frac{2}{2 \pi i} \oint_{C} \frac{f(s)}{\left(s-z_{0}\right)^{3}} d s
$$

De forma geral,

$$
f^{n}\left(z_{0}\right)=\frac{n !}{2 \pi i} \oint_{C} \frac{f(s)}{\left(s-z_{0}\right)^{n+1}} d s
$$

Rearranjando os termos,

$$
\frac{f^{n}\left(z_{0}\right)}{n !}=\frac{1}{2 \pi i} \oint_{C} \frac{f(s)}{\left(s-z_{0}\right)^{n+1}} d s
$$

nota-se que o lado esquerdo da equação (4.36) é igual ao valor do coeficiente $a_{n}$ da expansão em série de potências (4.28):

$$
a_{n}=\frac{f^{n}\left(z_{0}\right)}{n !}
$$

obtida a partir da comparação com a expansão em série de Taylor (4.29). Portanto, o valor do coeficiente da expansão em série de potências de uma função $f$ ao redor de um ponto $z_{0}$ é igual à respectiva derivada da integral de Cauchy no contorno de analiticidade $C$, do qual o ponto $z_{0}$ é interior, dividida por $n !$ :

$$
a_{n}=\frac{1}{2 \pi i} \oint_{C} \frac{f(s)}{\left(s-z_{0}\right)^{n+1}} d s
$$

Este resultado permite que se calcule os coeficientes de uma expansão em série quando se conhece os valores da função expandida apenas no contorno. Este desenvolvimento foi utilizado no contexto de água subterrânea por Otto Strack (48).

\subsubsection{Polinômios de Chebyshev}

Os polinômios de Chebyshev são dados pela fórmula (94)

$$
T_{n}(x)=\cos (n \arccos (x))
$$


Ao usar identidades trigonométricas, expressões explícitas para o polinômio são obtidas:

$$
\begin{aligned}
T_{0}(x) & =1 \\
T_{1}(x) & =x \\
T_{2}(x) & =2 x^{2}-1 \\
T_{3}(x) & =4 x^{3}-3 x \\
\cdots & \\
T_{n+1}(x) & =2 x T_{n}(x)-T_{n-1}(x), \quad n \geq 1
\end{aligned}
$$

Os polinômios de Chebyshev são ortogonais no intervalo $[-1,1]$, com peso $\frac{1}{\sqrt{1-x^{2}}}$, com zeros localizados em

$$
x=\cos \left(\frac{\pi\left(k+\frac{1}{2}\right)}{n}\right), \quad k=0,1,2 \ldots, n-1
$$

Eles também apresentam uma relação de ortogonalidade discreta: sendo os pontos $x_{k}$ zeros do polinômio $T_{m}(x)$ na forma (4.46) com $i, j<m$ :

$$
\sum_{k=0}^{m-1} T_{i}\left(x_{k}\right) T_{j}\left(x_{k}\right)=\left\{\begin{array}{l}
0, \quad i \neq j \\
m / 2, \quad i=j \neq 0 \\
m, \quad i=j=0
\end{array}\right.
$$

Para trabalhar com os polinômios de Chebyshev em domínios diferentes de $[-1,1]$, a transformação (4.4.1) pode ser usada $(1,94)$.

Estes resultados são válidos também para o caso em que o polinômio de Chebyshev é usado para variáveis complexas (95). No círculo unitário definido pela transformação (4.18), a substituição em (4.39) é

$$
z=\cos (\theta)
$$

e a partir de (4.27),

$$
\chi=Z+\sqrt{Z+1} \sqrt{Z-1}=e^{i \theta}
$$

portanto

$$
T_{n}(z)=\cos (n \theta)=\frac{1}{2}\left(e^{i n \theta}+e^{-i n \theta}\right)=\frac{1}{2}\left(\chi^{n}+\chi^{-n}\right) .
$$

A definição dos polinômios de Chebyshev em variáveis complexas é portanto igual a

$$
T_{n}(z)=\frac{1}{2}\left(\chi^{n}+\chi^{-n}\right)
$$


De forma direta,

$$
T_{n}(z)=\frac{1}{2}\left[(Z+\sqrt{Z+1} \sqrt{Z-1})^{n}+(Z-\sqrt{Z+1} \sqrt{Z-1})^{n}\right]
$$

Os polinômios de Chebyshev têm uma relação próxima com a série de Fourier. Dada a expansão padrão

$$
\lambda(x)=\sum_{n=0}^{\infty} a_{n} T_{n}(x)
$$

ao se fazer a substituição padrão $x=\cos (\theta)$ é obtida a expansão em série de Fourier:

$$
\lambda(\cos (\theta))=\sum_{n=0}^{\infty} a_{n} \cos (n \theta)
$$

As propriedades dos polinômios de Chebyshev serão usadas na expansão em série para as line doublet e para o elemento fratura. 


\section{$5 \quad$ A Inomogeneidade Circular, Line Doublet e a Fratura}

Os conceitos descritos nos capítulos anteriores serão usados neste capítulo para modelar um elemento circular com condutividade hidráulica diferente da do meio poroso, o elemento line doublet também chamado de linha dubleto $(51,55)$, para inomogeneidades poligonais, e uma das contribuições deste trabalho, o elemento para fraturas. As definições do elemento circular e da line doublet são baseadas no trabalho de Otto Strack, em livro a ser publicado (48). A solução exata para uma fratura é apresentada em (4), e estendida neste trabalho para um sistema de fraturas.

\subsection{Escoamento Uniforme}

Em todos os problemas aqui tratados supõe-se a existência de um campo de escoamento regional, uniforme, de intensidade $Q_{0}$ e formando um ângulo de $\beta_{\text {uf }}$ em relação ao eixo $x$. Cada elemento analítico é solução da equação de Laplace, a menos de uma constante aditiva. Esta constante é chamada de constante livre de referência, e é encontrada calculado-se seu valor em um ponto com um valor de carga hidráulica conhecida dentro do domínio de interesse. A constante livre de referência de todos os elementos é incluída na definição do escoamento uniforme por simplicidade. A definição do escoamento uniforme é (1)

$$
\Omega_{u f}=-Q_{0} z e^{-i \beta_{u f}}+C
$$

para o qual z é a coordenada global.

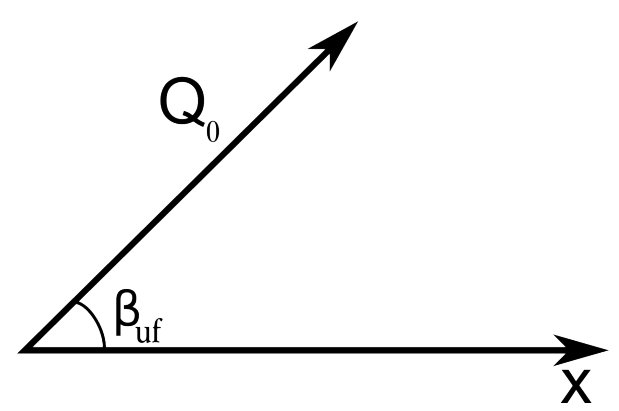

Figura 5.1 - Representação da direção do escoamento uniforme de intensidade $Q_{0}$ e direção $\beta_{u f}$ em relação ao eixo $x$. 


\subsection{Inomogeneidades Circulares}

Inomogeneidades circulares são regiões circulares com condutividade hidráulica $K^{+}$, diferente da condutividade do aquífero, $K$, podendo ser maior ou menor. Um nome mais correto seria inomogeneidades cilíndricas, já que são cilindros verticais de condutividade hidráulica $\mathrm{K}^{+}$. Como a aproximação de Dupuit-Forchheimer é usada, atém-se ao nome circular, dada a sua projeção no plano. A influência das inomogeneidades circulares são simuladas usando expansões em Série de Laurent no exterior e Série de Taylor na parte interna da inomogeneidade. A condição de contorno a ser imposta é que a carga deve ser contínua na borda da inomogeneidade $(1,93)$, onde a coordenada que descreve a borda da inomogeneidade é $\theta$ :

$$
\phi^{+}(\theta)=\phi^{-}(\theta)
$$

O potencial para uma inomogeneidade circular é igual a (93)

$$
\begin{array}{cc}
\Omega_{j}(Z)=\sum_{n=0}^{\infty} a_{n} Z_{j}^{n} & |Z| \leq 1 \\
\Omega_{j}(Z)=\sum_{n=0}^{\infty} a_{n} Z_{j}^{-n} & |Z|>1
\end{array}
$$

para o qual

$$
Z_{j}=\frac{z-z_{j}}{R^{j}}
$$

sendo $z_{j}$ o centro da inomogeneidade $j$ e $R_{j}$ o seu raio.

\subsubsection{Condição de Contorno}

Para satisfazer a condição de contorno para carga, o potencial deve ser descontínuo na borda, na forma

$$
\frac{\Phi^{+}}{K^{+}}=\frac{\Phi^{-}}{K}
$$

onde + significa a parte interna da inomogeneidade, e - a parte externa. A condição da descontinuidade do potencial leva à relação (5.8), com a mesma sendo calculada na borda do elemento, descrito pela variável $\theta, 0 \leq \theta \leq 2 \pi(93)$ :

$$
\frac{\Phi^{+}(\theta)}{K^{+}}=\frac{\Phi^{-}(\theta)}{K}
$$


Reescrevendo a expressão (5.8) como a combinação do potencial de todos os elementos (inclusive outras inomogeneidades) mais o potencial da inomogeneidade circular:

$$
\frac{\Phi_{\neq j}^{+}(\theta)+\Phi_{j}^{+}(\theta)}{K_{j}^{+}}=\frac{\Phi_{\neq j}^{-}(\theta)+\Phi_{j}^{-}(\theta)}{K}
$$

A única componente do potencial de descarga descontínua no contorno da inomogeneidade circular $j$ é a influência da própria inomogeneidade (93). Para os demais elementos $(\neq j)$,

$$
\begin{array}{r}
K \Phi_{\neq j}^{+}+K \Phi_{j}^{+}=K_{j}^{+} \Phi_{\neq j}^{-}+K_{j}^{+} \Phi_{j}^{-} \\
K \Phi_{j}^{+}-K^{+} \Phi_{j}^{-}=\left(K_{j}^{+}-K\right) \Phi_{\neq j}^{-} \Phi_{\neq j}^{+}(\theta)=\Phi_{\neq j}^{-}(\theta)=\Phi_{\neq j}(\theta)
\end{array}
$$

já que o potencial de descarga gerados pelos demais elementos é contínuo através da borda da inomogeneidade circular. A condição de contorno pode ser reescrita como

$$
K \Phi_{j}^{+}(\theta)-K_{j}^{+} \Phi_{j}^{-}(\theta)=\left(K_{j}^{+}-K\right) \Phi_{\neq j}^{+}(\theta)
$$

Esta expressão será usada para determinar os coeficientes da solução para a inomogeneidade circular $j$.

\subsubsection{Solução Exata para uma Inomogeneidade Circular e Escoamento Uniforme}

Strack (1) apresenta uma solução analítica para o problema de uma inomogeneidade circular em um campo e escoamento uniforme. O campo de escoamento uniforme tem intensidade $Q_{0}$ e é paralelo ao eixo $x$. A inomogeneidade tem raio $R$, com seu centro na origem. O potencial complexo para o interior do círculo é igual a

$$
\Omega^{+}=-Q_{0} \frac{2 K^{+}}{K+K^{+}} z+\Phi_{0}
$$

e para o exterior

$$
\Omega^{-}=-Q_{0}\left[z+\frac{K^{+}-K}{K^{+}+K} \frac{R^{2}}{z}\right]+\frac{K}{K^{+}} \Phi_{0}
$$

para o qual $\Phi_{0}$ é determinado em um ponto com carga conhecida. Esta solução analítica será usada como comparação para validação de soluções do MEA para inomogeneidades circulares e poligonais.

\subsection{Line Doublets e Inomogeneidades Poligonais}

Os elementos linha no MEA geralmente representam descontinuidades na parte real (o potencial de descarga $\Phi$ ) ou na parte imaginária (a função de corrente $\Psi$ ) do potencial complexo $\Omega$. São construídos como integrais de linha, na forma de integrais de Cauchy (4.4.3) e são obtidos integrandose dipolos ao longo da linha. Dada a forma geral do potencial $\Omega_{d p}$ de um dipolo de intensidade $s(z)$ 
e com ângulo $\beta$ em relação ao eixo $x$ (1):

$$
\Omega_{d p}(z)=\frac{s(z) e^{i \beta}}{z-s}
$$

a integração do mesmo ao longo da linha com ângulo $\alpha$ em relação ao eixo $x$ é igual ao potencial complexo $\Omega_{l d}$ :

$$
\Omega_{l d}(z)=\frac{1}{2 \pi} \int_{z_{1}}^{z_{2}} \frac{s(\delta) e^{i \beta}}{z-\delta} d \delta e^{-i \alpha}
$$

com $z_{1}$ e $z_{2}$, os pontos inicial e final da linha, respectivamente. Dependendo da orientação relativa entre o dipolo e a linha, o número complexo $i$ multiplica a integral. Se o dipolo está orientado de forma paralela à linha, o elemento obtido chama-se line dipole. Se orientado perpendicularmente à linha chama-se line doublet e são distintas apenas no termo $1 / i$ multiplicativo para a line doublet ${ }^{1}$. A forma tradicional de se construir a expressão para a line doublet é transformar a integral (5.15) usando a transformação para coordenadas locais lineares (4.4.1), obtendo-se uma integral na forma (1)

$$
\Omega(z)=\frac{1}{2 \pi i} \int_{-1}^{1} \frac{s(\Delta) e^{i(\beta-\alpha)}}{Z-\Delta} d \Delta
$$

O desenvolvimento a seguir segue o artigo (96) e Strack (48). Assume-se que a intensidade do dipolo seja expressa por uma expansão em série de potências na forma

$$
s(Z)=\lambda(Z)=\sum_{n=0}^{N} a_{n} Z^{n}
$$

com os coeficientes $a_{n}$ reais $\left(\mathfrak{I}\left(a_{n}\right)=0, \forall n\right)$. Substituindo na integral (5.16), com dipolo perpendicular à linha line doublet, obtém-se

$$
\Omega_{l d}(Z)=-\frac{1}{2 \pi i} \int_{-1}^{1} \frac{\sum_{n=0}^{N} a_{n} Z^{n}}{Z-\Delta} d(Z-\Delta)
$$

Executando esta integral termo a termo, o resultado é analítico e igual a

$$
\Omega_{l d}(Z)=\frac{1}{2 \pi i} \sum_{n=0}^{N} a_{n} Z^{n} \ln \frac{Z-1}{Z+1}+P(Z)
$$

na qual o termo $P(Z)$ é igual a

$$
P(Z)=\frac{1}{2 \pi i} \sum_{n=1}^{\infty} a_{n} \sum_{p=0}^{n-1} Z^{p}\left(\frac{1^{n-p}-(-1)^{n-p}}{n-p}\right)
$$

Barnes e Janković $(85,92)$ sugerem o uso de polinômios de Chebyshev como expansão da intensidade de dipolo $s(z)$, devido às propriedades de ortogonalidade do mesmo e a sua proximidade do polinômio minimax (94). Uma das vantagens em se usar polinômios de Chebyshev é que, ao

\footnotetext{
${ }^{1}$ Os nomes line dipole e line doublet são arbitrários.
} 
elevar o polinômio a alta ordem dentro do intervalo $[-1,1]$, o polinômio não vai a zero em todos os pontos do intervalo e a 1 na extremidade, como pode ser verificado nas figuras (5.2) e (5.3). Esta propriedade elimina o problema da oscilação do valores no intervalo, de se elevar em alta ordem a expansão em série de potências dentro do intervalo (85), como ocorre com o uso de colocação.

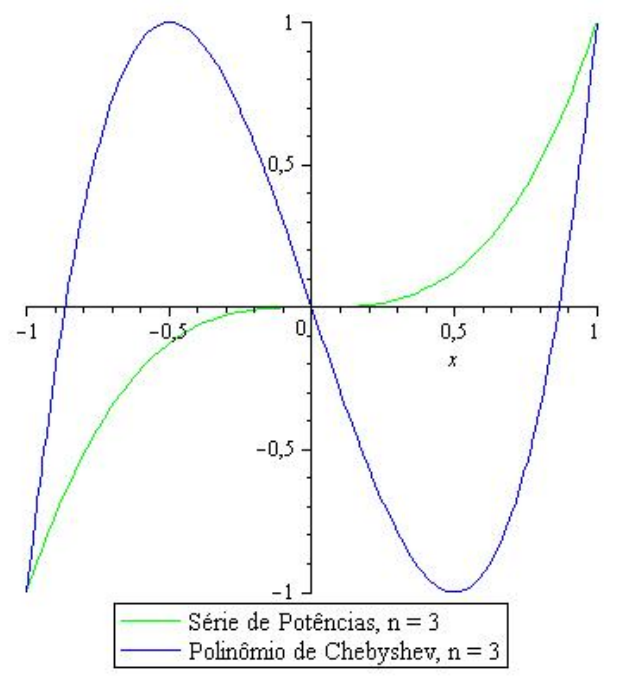

Figura 5.2 - Comparação entre a expansão em Polinômios de Chebyshev e em Série de Potências, para $n=3$.

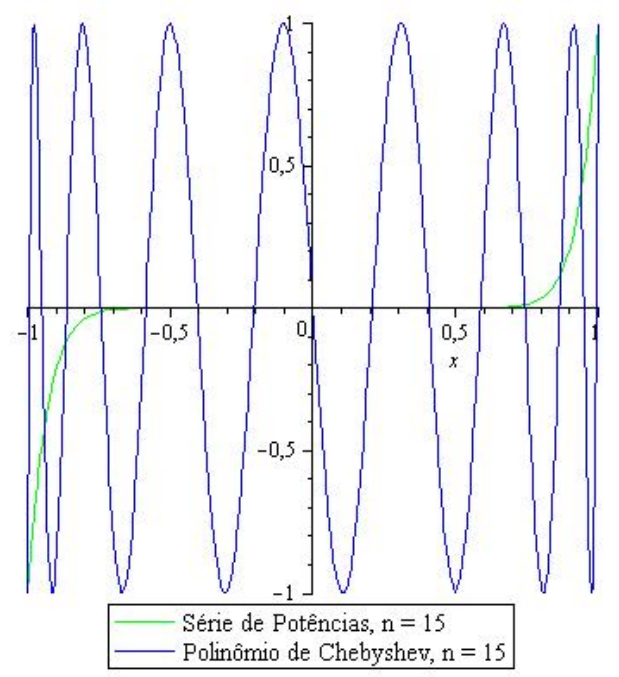

Figura 5.3 - Comparação entre a expansão em Polinômios de Chebyshev e em Série de Potências, para $n=15$. Nota-se que o Polinômio de Chebyshev preserva seu comportamento oscilatório no domínio, enquanto os termos para a Série de Potências são diferentes de zero apenas próximo às extremidades.

\subsubsection{Condições de Contorno}

As line doublet são usadas para modelar uma inomogeneidade delimitada por um polígono com condutividade hidráulica maior ou menor que a condutividade hidráulica do meio. As line doublet são conectadas nas extremidades, fechando o polígono. A condição de contorno de uma line doublet 
determina que a carga hidráulica deve ser contínua na interface, assim como para a inomogeneidade circular:

$$
\phi^{+}(x, y)=\phi^{-}(x, y)
$$

Para satisfazer esta condição de contorno, a parte real do potencial complexo é descontínua:

$$
\frac{\Phi^{+}\left(\delta_{l}\right)}{K^{+}}=\frac{\Phi^{-}\left(\delta_{l}\right)}{K}
$$

onde $\delta_{l}$ denota um ponto sobre a interface. As linhas de corrente $\Psi(z)$, a parte imaginária do potencial complexo $\Omega$, são contínuas através da interface. Para determinar a descontinuidade no potencial, os valores do mesmo são tomados de um lado e do outro do elemento, bem próximos deste, e são chamados de $\Omega^{+}$e $\Omega^{-}$, como pode ser visto na figura (5.4).

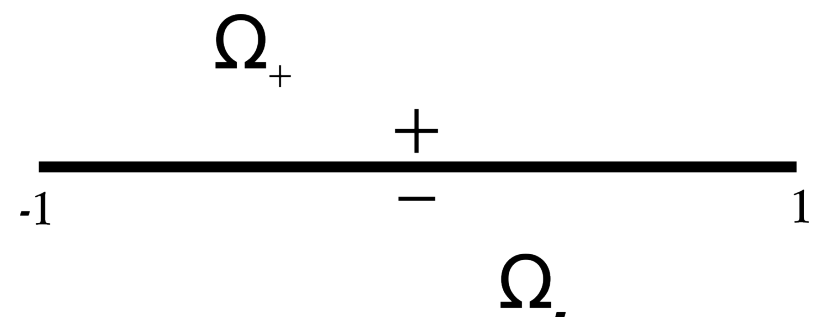

Figura 5.4 - Lados do elemento onde o potencial $\Omega_{+}$e $\Omega_{-}$são considerados.

Assumindo uma expansão em série de potências para a intensidade de dipolo $\lambda(Z)=\sum_{n=0}^{\infty} a_{n} Z^{n}$ e decompondo o potencial em partes real e imaginária, para cada lado da interface do elemento,

$$
\begin{aligned}
& \Omega^{+}(Z)=\frac{\sum_{n=0}^{\infty} a_{n} Z_{n}}{2 \pi i}\left(\ln \left|\frac{Z-1}{Z+1}\right|+i \theta^{+}+p_{n}(Z)\right) \\
& \Omega^{-}(Z)=\frac{\sum_{n=0}^{\infty} a_{n} Z_{n}}{2 \pi i}\left(\ln \left|\frac{Z-1}{Z+1}\right|+i \theta^{-}+p_{n}(Z)\right)
\end{aligned}
$$

Nota-se que a único termo que muda entre os dois lados do elemento são funções em $\theta$, dadas por

$$
\theta^{ \pm}=\arctan \left(\frac{Z-1}{Z+1}\right)
$$

Por causa do fator imaginário $i$ no denominador da expressão (5.23), a parte da descontinuidade no potencial é dada pela parte real, e expressa pela função $\theta^{ \pm}$. Os valores de $\theta^{ \pm}$próximos do ponto de um lado e do outro do elemento são iguais a

$$
\begin{array}{r}
\theta^{+}=\pi \\
\theta^{-}=-\pi
\end{array}
$$

Calculando a diferença de potencial entre ambos os lados,

$$
\mathfrak{I}\left(\Omega^{+}-\Omega^{-}\right)=\frac{\sum_{n=0}^{\infty} a_{n} Z_{n}}{2 \pi}\left(\theta^{+}-\theta^{-}\right)=\frac{\sum_{n=0}^{\infty} a_{n} Z_{n}}{2 \pi}(\pi-(-\pi))=\sum_{n=0}^{\infty} a_{n} Z_{n}=\lambda(Z)
$$


na qual

$$
\begin{array}{r}
\lambda_{j}^{+}(Z)=\frac{1}{2} \sum_{n=0}^{\infty} a_{n} Z_{n} \\
\lambda_{j}^{-}(Z)=-\frac{1}{2} \sum_{n=0}^{\infty} a_{n} Z_{n}
\end{array}
$$

Ou seja, a descontinuidade de potencial é igual à distribuição de densidade de dipolo, $\lambda$. Este resultado será usado para o cálculo dos coeficientes da expansão das line doublets.

\subsubsection{Definição do Potencial}

O uso da transformação (4.18) em conjunto com a a integral de Cauchy fornece uma poderosa ferramenta para calcular os coeficientes desconhecidos para cada elemento, dadas as condições de contorno. Ao utilizar a transformação (4.18), o elemento linha é levado no círculo unitário no sistema de coordenadas $\chi$. Neste sistema de coordenadas, o uso da integral de Cauchy para o cálculo dos coeficientes usando as condições de contorno é facilitada. No capítulo (6) esta transformação será discutida em mais detalhes.

Transformando a expressão da line doublet (5.19) para o sistema de coordenadas $\chi$ e assumindo a expansão em uma série de polinômios de Chebyshev para a intensidade de dipolo $\lambda(Z)$ da line doublet

$$
\lambda(\chi)=\frac{1}{2}\left[\chi^{n}+\left(\frac{1}{\chi}\right)^{n}\right]
$$

a expressão para o potencial é igual a

$$
\Omega_{n}(\chi)=a_{n} \frac{1}{2 \pi i} F_{n}(\chi)=a_{n} \frac{1}{4 \pi i}\left[\chi^{n}+\left(\frac{1}{\chi}\right)^{n}\right] \ln \frac{Z-1}{Z+1}+a_{n} \frac{p_{n}(\chi)}{2 \pi i}
$$

com a definição

$$
F_{n}(\chi)=\frac{1}{2}\left[\chi^{n}+\left(\frac{1}{\chi}\right)^{n}\right] \ln \frac{Z-1}{Z+1}+p_{n}(\chi)
$$

Transformando esta expressão das coordenadas lineares $Z$ para o plano $\chi$ através da transformação (4.21), obtém-se

$$
F_{n}(\chi)=\left[\chi^{n}+\left(\frac{1}{\chi}\right)^{n}\right] \ln \frac{\chi-1}{\chi+1}+p_{n}(\chi)
$$

O termo $p_{n}(\chi)$ é o termo de correção para campo distante. Campo distante é definido como o termo resultante da influência do potencial no infinito. Praticamente, é usado como uma distância suficientemente distante do elemento para que sua expressão possa ser simplificada, de forma a acelerar o cálculo de sua influência. Este termo deve garantir que $F_{n}(\chi)$ para campo distante é de ordem $O\left(\chi^{-1}\right)$ (48). Este termo é obtido expandindo o termo logaritmo em $F_{n}(\chi)$ para $|\chi|>>1$, multiplicando pelo polinômio de Chebyshev $T_{n}$, e determinando os coeficientes que tenham ordem 
maior que $O\left(\chi^{-1}\right)$. A expansão é igual a

$$
F_{n}(\chi) \approx-2 \chi^{n} \sum_{j=1}^{\infty} \beta_{j}\left(\frac{1}{\chi}\right)^{j}
$$

para a qual

$$
\begin{array}{ll}
\beta_{j}=\frac{1}{j}, & j=1,3,5 \ldots \infty \\
\beta_{j}=0, & j=2,4,6 \ldots \infty
\end{array}
$$

Considerando apenas os termos diferentes de zero, a correção deve cancelar os termos restantes da expansão (5.35), e portanto é igual a

$$
p_{n}(\chi)=2 \beta_{n}+2 \sum_{j=1}^{n-1} \beta_{n-j}\left[\chi^{j}+\left(\frac{1}{\chi}\right)^{j}\right]
$$

A expressão para o potencial da line doublet no sistema de coordenadas $\chi$ com a correção para campo distante é igual a

$$
F_{n}(\chi)=\left[\chi^{n}+\left(\frac{1}{\chi}\right)^{n}\right] \ln \frac{\chi-1}{\chi+1}+2 \beta_{n}+2 \sum_{j=1}^{n-1} \beta_{n-j}\left[\chi^{j}+\left(\frac{1}{\chi}\right)^{j}\right]
$$

Para simplificar o cálculo numérico desta expressão, diminuindo assim seu custo computacional, uma expansão do logaritmo ao redor do ponto $\frac{1}{\chi}=0$ é feita, sendo o termo $F_{n}(\chi)$ simplificado a

$$
F_{n}^{s}(\chi)=\sum_{j=1}^{\infty} \alpha_{j}\left(\frac{1}{\chi}\right)^{j}
$$

com

$$
\begin{array}{lr}
\alpha_{j}=2\left(\beta_{n-j}-\beta_{n+j}\right), & j=1, \ldots, n-1 \\
\alpha_{j}=-2 \beta_{2 n}, & j=n \\
\alpha_{j}=-2\left(\beta_{j-n}+\beta_{n+j}\right), & j=n+1, \ldots, \infty
\end{array}
$$

Nota-se que esta expansão tem o formato de uma expansão em série de Laurent, com o comportamento esperado no infinito. A expressão do potencial para uma line doublet é igual a

$$
\Omega_{k}(\chi)=\sum_{n=0}^{\infty} a_{k, n} F_{k, n}(\chi)
$$

onde agora o índice $k$ denota uma line doublet em particular. A transição entre o campo próximo e o campo distante é da ordem de $1<|\chi|<2$. Quanto mais próximo de 1 , mais rapidamente a função $F_{n}^{s}$ pode ser usada, reduzindo o custo computacional do cálculo do potencial. 


\subsection{O Elemento Analítico para Fraturas}

A solução exata para uma fratura veio do Relatório Battelle, de Otto Strack (4). O princípio básico é que a fratura teria um formato elipsóide, com fluxo zero nas extremidades e condutividade hidráulica maior que a do meio no seu interior. As condições de contorno seriam satisfeitas de forma aproximada na borda da fratura, considerando que a fratura, vista de fora, do meio, é aproximada por uma linha, como pode ser visto na figura (5.7). Esta configuração é originária do deslocamento linear de um material elástico infinito (97).

Como visto no capítulo (3), a modelagem de fraturas é complicada por diversos fatores. Foi feita neste trabalho a opção de usar a simplificação da lei linear para a abertura, com a fratura preenchida por um material com condutividade hidráulica maior que a do meio. A primeira justificativa para esta simplificação consiste em considerar que para as escalas para as quais o MEA foi proposto, fenômenos como rugosidade das paredes e variação de abertura na mesma fratura não são importantes para a descrição do escoamento. A segunda justificativa é que a parametrização utilizada, com a abertura e a condutividade hidráulica da fratura como parâmetros, pode incluir uma gama de problemas interessantes em sistemas fraturados. Com a flexibilidade do MEA para a variação de escala sem a necessidade de rediscretizar o domínio e de recalcular a solução para aumentar a precisão da mesma, a simplificação na descrição geométrica da fratura é compensada.

Uma outra característica importante do modelo escolhido é a integração da rede de fraturas com o meio poroso, havendo escoamento em ambos, o que não seria possível em uma rede discreta sem comunicação com o meio poroso. A abordagem escolhida permite simular características de redes discretas, por incluir cada fratura de forma independente. Este modelo é, portanto, classificado como um modelo para escoamento em rocha porosa fraturada (26). A figura (5.5) mostra um esquema para uma fratura com abertura $b$, e a figura (5.6) mostra o formato elipsóide da fratura utilizada neste trabalho.

O elemento analítico fratura foi originalmente desenvolvido para uma fratura isolada, interagindo somente com o escoamento uniforme. Um dos avanços desta tese é o aperfeiçoamento deste elemento para várias fraturas que interagem com o escoamento uniforme e entre si, e com o formalismo permitindo interação com todos os outros elementos da família dos elementos analíticos.

\subsubsection{Condições de Contorno}

As condições de contorno para uma fratura de abertura $b$ e representada por uma linha unidimensional são

- A fratura não extrai água do aquífero,

- As linhas de corrente são descontínuas de forma transversal ao elemento, com descontinuidade igual a $\mu$,

- A carga é constante transversalmente ao elemento. 


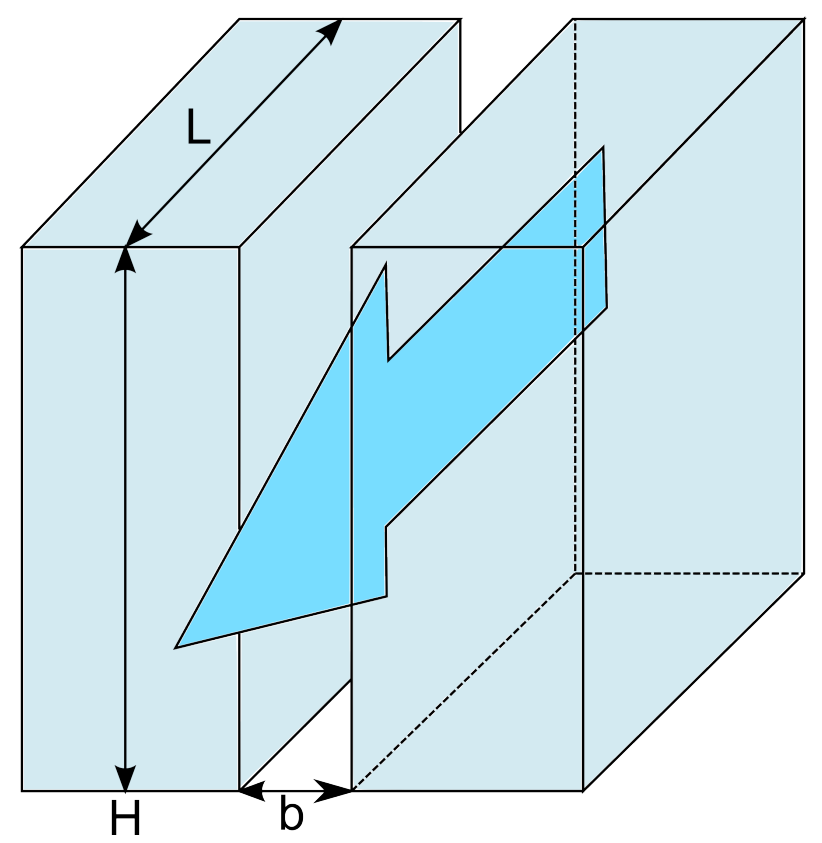

Figura 5.5 - Esquema de uma fratura vertical comprimento $L$, altura $H$ e abertura $a$. Adaptado de (3).

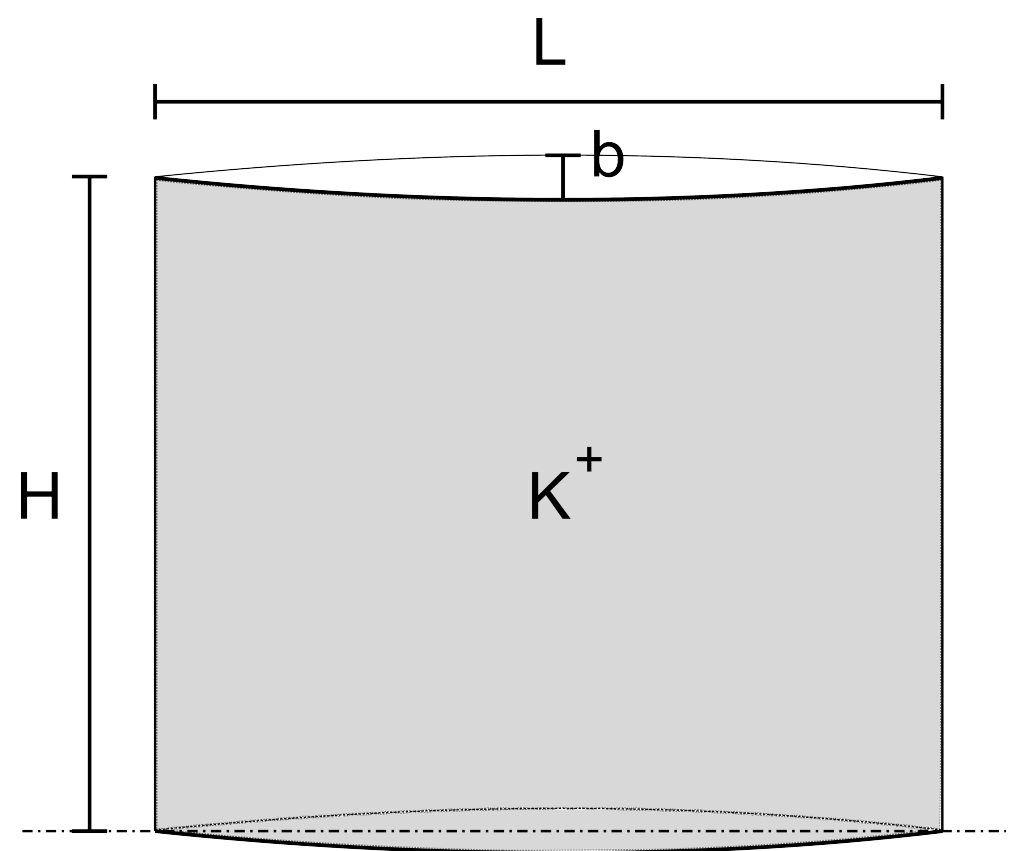

Figura 5.6 - Esquema de uma fratura vertical com abertura $b$, condutividade hidráulica $K^{+}$, comprimento $L$ e altura $H$. Este é o formato da fratura utilizada neste trabalho.

A fratura é vertical, com altura $H$, e abertura $b$. O sistema de coordenadas orientado em relação à fratura é formado pelos eixos $s$ e $n$, onde $s$ é paralelo à fratura e $n$, ortogonal. 

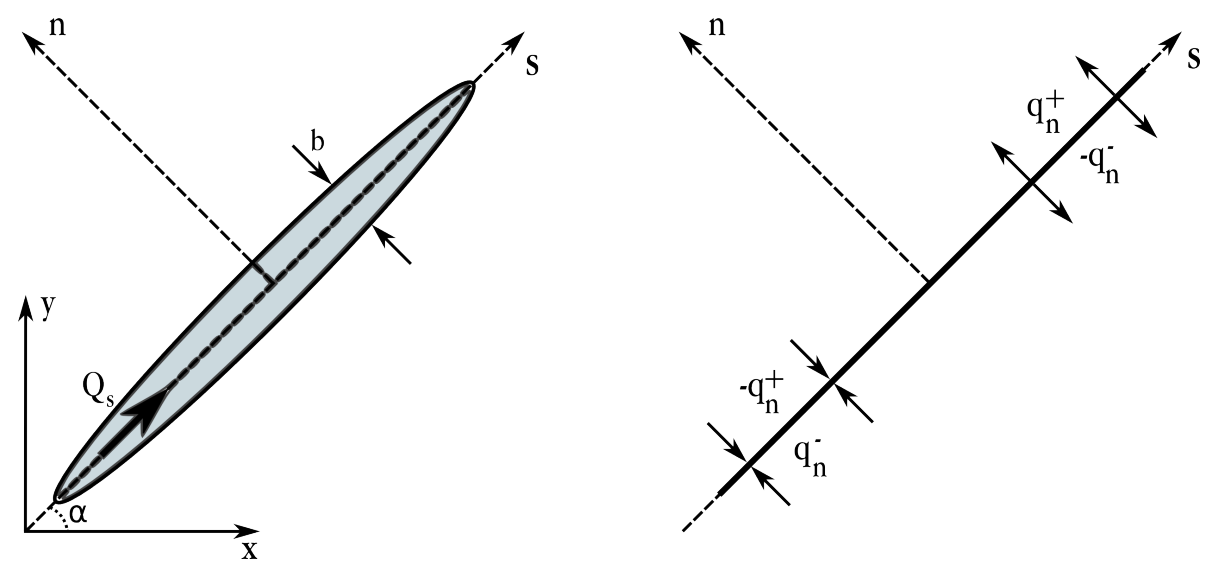

Figura 5.7 - Fratura. Escoamento visto de dentro da fratura (esquerda) e do meio rochoso (direita). Adaptado de (4).

Como visto na seção sobre a lei cúbica (3.5.1), a dinâmica do escoamento é igual à relação entre o vetor descarga $Q_{s}$ e o gradiente da carga na direção da fratura $s$ :

$$
Q_{s}=-\beta b^{3} \frac{\partial \overline{\phi(s, n)}}{\partial s}
$$

onde $\overline{\phi(s, n)}$ é a carga média transversalmente à fratura. A lei linear para a dinâmica da fratura é igual a

$$
Q_{s}=-\frac{K^{+} b}{K} \frac{\partial \overline{\phi(s, n)}}{\partial s}
$$

que é usada neste trabalho, em que a fratura é considerada como preenchida por um material poroso de condutividade hidráulica $K^{+}$. A continuidade de fluxo na fratura requer que o vetor de descarga $Q_{s}$ na fratura, $-\frac{L}{2} \leq s \leq \frac{L}{2}$ seja igual ao influxo total na fratura:

$$
Q_{s}=\int_{\frac{-L}{2}}^{s} \sigma(s) d t
$$

na direção $s$ da fratura. $\sigma(s)$ é igual à soma do influxo de ambos os lados da fratura, por unidade de comprimento da fratura, e pode ser descrito pela diferença do vetor descarga específica na direção normal à fratura em ambos os lados da mesma:

$$
\sigma(s)=-\left[q_{n}^{+}-q_{n}^{-}\right]
$$

Assume-se ao longo da fratura que carga média $\bar{\phi}$ é igual à carga fora da fratura, $\phi$, na direção transversal à mesma,

$$
\overline{\phi(s, n)}=\phi(s, n), \quad-\frac{L}{2} \leq s \leq \frac{L}{2}, \quad n=0
$$


A equação (5.43) pode ser expressa em função do potencial de descarga $\Phi$, multiplicando e dividindo a condutividade hidráulica do meio $K$ e usando a definição do potencial:

$$
Q_{s}=-\frac{K^{+} b}{K} \frac{\partial K \overline{\phi(s, n)}}{\partial s}=-\frac{K^{+} b}{K} \frac{\partial \Phi(s, n)}{\partial s}
$$

O fluxo de água que passa na direção $s$ da fratura também é igual à diferença das linhas de corrente em sua borda:

$$
Q_{c}=-\left(\Psi^{+}(x, y)-\Psi^{-}(x, y)\right)
$$

O vetor de descarga na direção da fratura, $Q_{s}$, é igual a

$$
Q_{s}=-\frac{d \Phi}{d s}
$$

Combinando as expressões (5.47) a (5.49), obtém-se

$$
Q_{s}=-\frac{k}{k^{*} b}\left(\Psi^{+}-\Psi^{-}\right)=-\frac{d \Phi}{d s}
$$

Verifica-se que a descontinuidade na função de corrente é igual ao esforço $\mu$, definido como

$$
\mu=-\left(\Psi^{+}-\Psi^{-}\right)
$$

Portanto, a expressão final para a condição de contorno na fratura, ao longo de seu comprimento, é igual a

$$
\mu=\frac{K^{+} b}{K} \frac{d \Phi}{d s}
$$

O vetor descarga para um elemento analítico, em geral, é dado pela derivada do potencial complexo:

$$
W(z)=-\frac{d \Omega(z)}{d z}=Q_{x}(z)-i Q_{y}(z)
$$

no plano $z$, para o qual $Q_{x}(z)$ e $Q_{y}(z)$ são as descargas nas direções $x$ e $y$ respectivamente. Usando a regra da cadeia, o vetor descarga no plano $Z$ é igual a

$$
W(z)=-\frac{d \Omega(z)}{d z}=-\frac{d \Omega(Z)}{d Z} \frac{d Z}{d z}
$$

para o qual a derivada $\frac{d Z}{d z}$ para a transformação (4.4.1) fornece

$$
\frac{d Z}{d z}=\frac{2}{z_{2}-z_{1}}
$$

Portanto o vetor descarga é igual a

$$
W(z)=-\frac{d \Omega(Z)}{d Z} \frac{2}{z_{2}-z_{1}}
$$


A parte real da derivada do potencial de descarga na direção paralela à fratura, $s$, é igual ao vetor descarga do elemento (5.56), rotacionada para direção da fratura $s$ :

$$
\frac{d \Phi(z)}{d s}=\Re\left(W(z) e^{i \alpha_{i}}\right)
$$

A descontinuidade do potencial, portanto, é igual a

$$
\mu=\frac{K_{i}^{+} b_{i}}{K} \Re\left(W(z) e^{i \alpha_{i}}\right)=\Psi^{+}-\Psi^{-}
$$

\subsubsection{Solução Analítica para uma Fratura}

A abertura $b$ é definida como o espaçamento entre as paredes da fratura. O formato da fratura é elipsóide, como visto na figura (5.7), de acordo com (97), e o parâmetro $b$ é definido como

$$
b=b^{*} \sqrt{Z^{2}-1}
$$

onde $b^{*}$ é a largura da fratura no centro, onde $X=0$, sendo o valor de abertura máxima da fratura. Usando o sistema de coordenadas local $Z$, a abertura $b$ é dada em função de $X$ :

$$
b=b^{*} \sqrt{1-X^{2}}
$$

Para uma fratura obedecendo a lei cúbica esta relação é igual a

$$
b=b^{*}\left(1-X^{2}\right)^{\frac{1}{6}}
$$

Calculando a expressão da abertura $b$ da fratura usando a transformação de coordenadas para o plano $\chi$, (4.21), obtém-se

$$
\begin{array}{r}
\left(e^{i \theta}-e^{-i \theta}\right)=2\left(\sqrt{X^{2}-1}\right) \\
2 i \sin (\theta)=2 i\left(\sqrt{1-X^{2}}\right) \\
\sin (\theta)=\left(\sqrt{1-X^{2}}\right) \\
b=b^{*}\left(\sqrt{1-X^{2}}\right)=b^{*} \sin (\theta)=b^{*} \sqrt{1-\Re(Z)^{2}}
\end{array}
$$

Ao descrever a forma da fratura usando esta abertura elipsóide, deve-se observar que, visto de dentro da fratura, a forma da mesma é dada por (5.62d), enquanto vista do meio poroso, a forma da fratura é descrita por uma linha, onde a condição de contorno é imposta.

Uma solução para a condição de contorno (5.57) é a função complexa

$$
\Omega(Z)=A(Z+\sqrt{Z+1} \sqrt{Z-1})
$$

proposta por Strack em (4). Esta função satisfaz as condições de contorno descritas por (5.58) e é originária de modelos de descontinuidade de deslocamento em meios elásticos semi-infinitos (97). Para a solução do escoamento em uma fratura em um campo de escoamento uniforme a constante $A$ 
deve ser calculada. Para tanto, o campo de escoamento uniforme deve ser convertido para o sistema de coordenadas local $Z$ (descrita na seção (4.4.1)):

$$
\Omega_{u f}(Z)=-Q_{0} \frac{1}{2} L e^{i(\alpha-\beta)} Z+C
$$

para a qual o comprimento da fratura, $L$, é dado por

$$
L=\left(z_{2}-z_{1}\right)\left(\overline{z_{2}-z_{1}}\right)
$$

Substituindo o potencial na equação (5.64) na expressão para a condição de contorno (5.57) encontrase que a constante $A$ é igual a

$$
A=\frac{\frac{1}{2} K^{+} b^{*}}{k L+K^{+} b^{*}} Q_{0} L \cos (\alpha-\beta)
$$

O potencial final é igual a

$$
\Omega(Z)=\frac{\frac{1}{2} K^{+} b^{*}}{k L+K^{+} b^{*}} Q_{0} L \cos (\alpha-\beta)(Z+\sqrt{Z+1} \sqrt{Z-1})-Q_{0} \frac{1}{2} L e^{i(\alpha-\beta)} Z+C
$$

com a constante $C$ devendo ser calculada em um ponto de referência de carga conhecida, afastado do domínio de interesse. A solução (5.67) é exata e descreve o potencial de uma fratura de condutividade hidráulica $K^{+}$e abertura $b$, com ponto inicial $z_{1}$ e ponto final $z_{2}$, comprimento $L$, orientada com o ângulo $\alpha$ em relação ao eixo $x$, imersa em um campo de escoamento uniforme de intensidade $Q_{0} \mathrm{e}$ ângulo $\beta_{u f}$ com o eixo $x$. O potencial complexo (5.67) será usado como base para o potencial para várias fraturas.

\subsubsection{Descontinuidade no Potencial da Fratura}

Para entender o comportamento do potencial de descarga e seus ramos, é útil descrever a fratura no plano $Z$. A equação do potencial para fratura (5.63) pode ser reescrita como

$$
\sqrt{(Z-1)(Z+1)}=\sqrt{(X+i Y-1)(X+i Y+1)}
$$

e deve ser tratada com cuidado, notando que o termo $\sqrt{X^{2}-1}$ é sempre imaginário, se $X \leq 1$ e pode ser trocado por $i \sqrt{1-X^{2}}$ :

$$
-\infty \leq X \leq-1 \quad Y=0\left\{\begin{array}{l}
\Phi=\sqrt{X^{2}} \cos (\theta)-\sqrt{1-X^{2}} \sin \left(\theta_{1}+\theta_{2}\right) \\
\Psi=\sqrt{X^{2}} \sin (\theta)-\sqrt{1-X^{2}} \cos \left(\theta_{1}+\theta_{2}\right)
\end{array}\right.
$$

A descontinuidade do potencial transversalmente ao elemento é dada por

$$
-1 \leq X \leq 1 \quad Y=0\left\{\begin{array}{l}
\Phi=\sqrt{X^{2}} \cos (\theta)-\sqrt{1-X^{2}} \sin \left(\theta_{1}+\theta_{2}\right) \\
\Psi=\sqrt{X^{2}} \sin (\theta)-\sqrt{1-X^{2}} \cos \left(\theta_{1}+\theta_{2}\right)
\end{array}\right.
$$




$$
1 \leq X \leq \infty \quad Y=0\left\{\begin{array}{l}
\Phi=\sqrt{X^{2}} \cos (\theta)-\sqrt{X^{2}-1} \cos \left(\theta_{1}+\theta_{2}\right) \\
\Psi=\sqrt{X^{2}} \sin (\theta)-\sqrt{X^{2}-1} \sin \left(\theta_{1}+\theta_{2}\right)
\end{array}\right.
$$

nos quais os ângulos são definidos como

$$
\begin{array}{r}
\theta=\arctan \left(\frac{Y}{X}\right) \\
\theta_{1}=\arctan \left(\frac{Y}{X-1}\right) \\
\theta_{2}=\arctan \left(\frac{Y}{X+1}\right)
\end{array}
$$

e a convenção de sinais segue a função $\operatorname{atan} 2^{2}$. Com estas definições, a descontinuidade $\Psi^{+}(X)-$ $\Psi^{-}(X)$ transversalmente ao elemento é igual a

$$
-1 \leq X \leq 1 \quad Y=0 \quad \Psi^{+}(X)-\Psi^{-}(X)=-2 a_{1} \sqrt{1-X^{2}}
$$

e usando o coeficiente $A$ para uma fratura:

$$
-1 \leq X \leq 1 \quad Y=0 \quad \Psi^{+}(X)-\Psi^{-}(X)=-\frac{k^{*} b^{*}}{k L+k^{*} b^{*}} Q_{0} L \cos (\beta-\alpha) \sqrt{1-X^{2}}
$$

No plano da fratura $Z$, a descarga $Q_{s}$ é zero em ambas as extremidades. A descarga na direção da fratura, dentro da mesma, é igual a

$$
-1 \leq X \leq 1 \quad Y=0 \quad Q_{c}=\frac{k^{*} b^{*}}{k L+k^{*} b^{*}} Q_{0} L \cos (\beta-\alpha) \sqrt{1-X^{2}}
$$

O vetor descarga, na direção da fratura, e dentro da mesma, é igual a

$$
-1 \leq X \leq 1 \quad Y=0 \quad Q_{X}=-\frac{d \Phi}{d X}=a_{1}-Q_{0} \frac{L}{2} \cos (\beta-\alpha)
$$

\subsubsection{Potencial Complexo para mais de uma Fratura}

Neste trabalho o potencial (5.67) foi adaptado para várias fraturas. Ao invés de ter apenas uma incógnita, $A$, o potencial foi expandido em uma série:

$$
\Omega_{j}(Z)=\sum_{n=1}^{\infty} a_{j n}\left(Z-\sqrt{Z_{j}+1} \sqrt{Z_{j}-1}\right)^{n}
$$

O índice $j$ representa o potencial complexo da fratura $j$. O potencial do elemento é zero no infinito: para o limite de $Z \rightarrow \infty$, o potencial tende a zero, não necessitando um polinômio corretor, como no

\footnotetext{
${ }^{2} \mathrm{~A}$ função atan2 é uma função definida na maioria das linguagens de programação. O intervalo da função é dado por $[-\pi, \pi]$.
} 
caso da line doublet :

$$
\lim _{Z \rightarrow \infty} \sum_{n=1}^{\infty} a_{n}(Z-\sqrt{Z+1} \sqrt{Z-1})^{n}=0
$$

O potencial total para um conjunto de fraturas é dado por

$$
\Omega(Z)=\sum_{j=1}^{M} \sum_{n=1}^{N} a_{j n}\left(Z_{j}-\sqrt{Z_{j}+1} \sqrt{Z_{j}-1}\right)^{n}
$$

para o qual $M$ é o número de fraturas presentes no domínio e $N$ a ordem de truncamento da expansão em série do potencial. 


\section{Algoritmos de Solução e Implementação Numérica}

\subsection{Introdução}

As condições de contorno no MEA são satisfeitas de forma aproximada, apesar das soluções da equação governante serem exatas. Um dos objetivos ao se satisfazer as condições de contorno é minimizar o erro e o custo computacional ao mesmo tempo, aumentando a precisão das soluções tanto no aspecto numérico quanto no comparativo a valores experimentais. Diferentes estratégias foram usadas para satisfazer as condições: o método de colocação, que resulta em uma matriz a ser diagonalizada; sobreespecificação, que aplica mínimos quadrados, podendo resultar tanto num sistema com matriz retangular como na soma de diversas contribuições para cada elemento da matriz; e no uso de integrais numéricas para calcular os elementos da matriz, tanto no sentido de mínimos quadrados quanto no produto de funções ortogonais. Métodos iterativos de solução, sem a necessidade de construção explícita da matriz, também são empregados, com variado grau de sucesso. Como é comum em abordagens numéricas, ganhos na precisão dos resultados têm um maior custo computacional. Uma técnica numérica efetiva é aquela que encontra um balanço entre a precisão requerida e custo computacional necessário para tal.

Um aspecto interessante da aplicação de técnicas numéricas em Geociências, e que é válido para o MEA, é que muitas vezes a abordagem numérica é muito mais precisa do que os dados adquiridos em campo ${ }^{1}$. De fato, mesmo se a abordagem numérica for altamente precisa, se os dados de campo não forem de precisão compatível, o modelo é limitado pela precisão de campo e não pela exatidão numérica. O uso indiscriminado de modelos numéricos, sem o entendimento dos mesmos e de suas limitações acaba levando a modelos errados ou mesmo irreais.

O uso de colocação já foi extensivamente abordado na literatura e não será revisto (ver referências (55) e (51) para aplicações do mesmo). O método iterativo sem a construção explícita da matriz será detalhado e aplicado para o caso de inomogeneidades circulares e poligonais. A falha deste método iterativo para o caso de fraturas motivou uma volta para os métodos matriciais explícitos e um estudo de convergência para métodos iterativos de solução da matriz. A aplicação do método matricial foi feita para as inomogeneidades poligonais e para a fratura, usando tanto uma abordagem de mínimos quadrados e uma de integração de polinômios ortogonais, que, inclusive, são similares,

\footnotetext{
${ }^{1}$ Edson Wendland, comunicação pessoal
} 
como será explicado no texto.

Após uma exaustiva tentativa de adaptar o algoritmo iterativo ao problema das fraturas, os resultados continuavam na grande maioria dos casos divergente. Tal divergência foi o que motivou a adaptação do processo iterativo para um método matricial. A razão da escolha de um método matricial vem da possibilidade de estudar a forma da matriz resultante e entender a convergência da solução, de acordo com o método escolhido para a solução do sistema linear. O número de condição, a norma e o raio espectral da matriz servem como ferramentas para analisar o comportamento do algoritmo de solução do sistema linear de forma iterativa.

\subsection{Condições de Contorno e a Integral de Cauchy}

Na seção (4.4.4) foi demonstrada a relação entre os coeficientes de uma expansão em Série de Taylor de uma função analítica $f$ e a Integral de Cauchy no contorno $C$ onde a função $f$ é analítica. Esse desenvolvimento será usado para calcular os valores dos coeficientes da expansão de $f$. É possível calcular os valores de coeficientes da expansão de $f$ usando os valores que forem conhecidos da parte real e da parte imaginária de $f$ no contorno $C$.

\subsubsection{Forma Geral}

A forma de calcular coeficientes de uma expansão de uma função em série de Taylor usando a parte real ou imaginária da função expandida é geral e serve de guia para as aplicações específicas para cadas elemento. A função analítica $H(\chi)$ e sua expansão em série de Taylor no plano $\chi$ é igual a

$$
H(\chi)=\Xi+i \Upsilon=\sum_{n=1}^{\infty} d_{n} \chi^{n}=\sum_{n=1}^{\infty} \frac{d^{n} H}{d \chi^{n}} \chi^{n}
$$

possibilita o uso da relação entre os coeficientes da Série de Taylor e a integral de Cauchy, com os coeficientes $d_{n}$ sendo calculados por

$$
d_{n}=\frac{1}{2 \pi i} \int_{0}^{2 \pi} \frac{H(\delta)}{\delta-\chi} d \delta
$$

Strack (48) descreve uma abordagem para o cálculo dos coeficientes quando apenas a parte real ou a parte imaginária da função $H$ é conhecida. Considerando a expansão $\chi$ ao redor de zero, $\chi=0$, têm-se um caso especial:

$$
\frac{1}{n !} \frac{d^{n} H(0)}{d \chi^{n}}=d_{n}=\frac{1}{2 \pi i} \int_{0}^{2 \pi} \frac{H(\delta)}{\delta^{n+1}} d \delta
$$

Usando o teorema de Cauchy-Goursat, a integral

$$
\frac{1}{2 \pi i} \oint_{C} H(\delta) \delta^{n} d \delta=0, \quad \forall n
$$


é igual a zero quando $\delta$ for analítica em $C$. O complexo conjugado de uma integral que é zero, também é zero:

$$
\frac{1}{2 \pi i} \oint_{C} \overline{H(\delta) \delta^{m}} d \bar{\delta}=0, \quad \forall m
$$

Considerando $\chi$ percorrendo o círculo unitário, onde a relação $\bar{\delta}=\delta^{-1}$ vale, é possível trocar de variável a integral (6.5), obtendo

$$
\frac{1}{2 \pi i} \oint_{C} \overline{H(\delta)} \frac{1}{\delta^{m+2}} d \delta=0
$$

Escolhendo $m-1=n$ e somando (6.4) com a equação (6.6), para $n>1$, obtém-se

$$
d_{n}=\frac{1}{2 \pi i} \oint_{C} \frac{H(\delta)+\overline{H(\delta)}}{\delta^{n+1}}
$$

Nota-se que a soma de uma função complexa e seu complexo conjugado é igual à parte real da mesma,

$$
d_{n}=\frac{1}{\pi i} \oint_{C} \frac{\Xi(\delta)}{\delta^{n+1}}, \quad n>1
$$

Com esta expressão é possível calcular os coeficientes da expansão em série de $H$ conhecendo apenas a parte real da mesma em sua borda. O mesmo desenvolvimento vale para a parte imaginária, se, ao invés de somar (6.4) com a equação (6.6) elas forem subtraídas:

$$
d_{n}=\frac{1}{\pi} \oint_{C} \frac{\Upsilon(\delta)}{\delta^{n+1}}, \quad n>1
$$

Esse desenvolvimento geral pode ser usado para encontrar o valor dos coeficientes para cada elemento. Outras abordagens, como o uso da ortogonalidade diretamente nas condições de contorno, fornecem resultados semelhantes.

\subsection{Inomogeneidades Circulares}

\subsubsection{Ortogonalidade da Série de Fourier}

A condição de contorno (5.2) para a inomogeneidade circular é usada para calcular os valores dos coeficientes desconhecidos da expansão do potencial para o elemento analítico em questão. $\mathrm{O}$ cálculo dos coeficientes desconhecidos d equação (5.3) pode ser abordado primeiramente usando a relação de ortogonalidade de Fourier. Tomando a parte real da condição de contorno (5.2) e usando o potencial para a inomogeneidade circular (5.3) no contorno da inomogeneidade, onde $Z=e^{i \theta}$

$$
K \Re\left(\sum_{n=0}^{\infty} a_{n} e^{i n \theta}\right)-K_{j}^{+} \Re\left(\sum_{n=0}^{\infty} a_{n} e^{-i n \theta}\right)=\left(K_{j}^{+}-K\right) \Phi_{\neq j}(\theta)
$$


Multiplicando em ambos os lados por $e^{-i m \theta}$, obtém-se

$$
\left[K \Re\left(\sum_{n=0}^{\infty} a_{n} e^{i n \theta}\right)-K_{j}^{+} \Re\left(\sum_{n=0}^{\infty} a_{n} e^{-i n \theta}\right)\right] e^{-i m \theta}=\left(K_{j}^{+}-K\right) \Phi_{\neq j}(\theta) e^{-i m \theta}
$$

As relações de ortogonalidade podem ser obtidas usando a identidade de Euler,

$$
e^{-i m \theta}=\cos (m \theta)-i \sin (m \theta)
$$

com

$$
K \Re\left(\sum_{n=0}^{\infty} a_{n} e^{i n \theta}\right)[\cos (m \theta)-i \sin (m \theta)]-K_{j}^{+} \Re\left(\sum_{n=0}^{\infty} a_{n} e^{-i n \theta}\right) \begin{array}{r}
{[\cos (m \theta)-i \sin (m \theta)]=} \\
\left(K_{j}^{+}-K\right) \Phi_{\neq j}(\theta) e^{-i m \theta}
\end{array}
$$

e

$$
\begin{array}{r}
K \sum_{n=0}^{\infty}\left(\Re\left(a_{n}\right) \cos (n \theta)-\mathfrak{I}\left(a_{n}\right) \sin (n \theta)\right)[\cos (m \theta)-i \sin (m \theta)]+ \\
K_{j}^{+} \sum_{n=0}^{\infty}\left(\Re\left(a_{n}\right) \cos (n \theta)+\mathfrak{S}\left(a_{n}\right) \sin (n \theta)\right)[\cos (m \theta)-i \sin (m \theta)]= \\
\left(K_{j}^{+}-K\right) \Phi_{\neq j}(\theta) e^{-i m \theta}
\end{array}
$$

multiplicando termo a termo e integrando $\theta$ no intervalo de 0 a $2 \pi$, usando as relações de ortogonalidade do Anexo (D), obtém-se

$$
\begin{array}{r}
a_{0}=\frac{k_{j}^{+}-K}{K} \frac{1}{2 \pi} \int_{0}^{2 \pi} \Phi_{\neq j}(\theta) d \theta \\
a_{n}=\frac{k_{j}^{+}-K}{K+k_{j}^{+}} \frac{1}{2 \pi} \int_{0}^{2 \pi} \Phi_{\neq j}(\theta) e^{-i n \theta} d \theta
\end{array}
$$

Estas integrais podem ser resolvidas usando métodos numéricos, como o pacote Quadpack incluso no Scipy (98). Por causa do uso de polinômios ortogonais na expansão das integrais, a abordagem de mínimos quadrados é similar, como descrito a seguir.

\subsubsection{Mínimos Quadrados}

Usando uma abordagem de mínimos quadrados, a equação a ser minimizada é construída a partir da equação (5.8):

$$
\sum_{m=0}^{M-1}\left[K \Phi_{j}^{+}-k_{j} \Phi_{j}^{-}-\left(k_{j}-k\right) \Phi_{\neq j}\right]^{2} \rightarrow \operatorname{MIN}\left[a_{0}, \ldots, a_{n}\right]
$$

Os dois primeiros termos são considerados desconhecidos e serão calculados. O último termo à direita , $\Phi_{\neq j}(\theta)$, é considerado conhecido, sendo a soma da contribuição de todos os outros elementos presentes. Substituindo a equação para o potencial, diferenciando esta expressão com respeito 
aos coeficientes desconhecidos $a_{n}$ e igualando a zero, obtém-se um sistema de equações para cada incógnita. Por exemplo, usando a expressão

$$
\begin{array}{r}
\sum_{m=0}^{M-1}\left[K \sum_{n=0}^{\infty}\left(\Re\left(a_{n}\right) \cos \left(n \theta_{m}\right)-\mathfrak{I}\left(a_{n}\right) \sin \left(n \theta_{m}\right)\right)+\right. \\
\left.K_{j}^{+} \sum_{n=0}^{\infty}\left(\Re\left(a_{n}\right) \cos \left(n \theta_{m}\right)+\mathfrak{I}\left(a_{n}\right) \sin \left(n \theta_{m}\right)\right)-\left(K_{j}^{+}-K\right) \Phi_{\neq j}(\theta) e^{-i m \theta_{m}}\right]^{2} \rightarrow M I N\left[a_{0}, \ldots, a_{n}\right]
\end{array}
$$

e derivando com relação à $\Re\left(a_{k}\right)$, a equação para mínimos quadrados fica igual a

$$
\begin{array}{r}
\frac{\partial}{\partial \Re\left(a_{k}\right)} \sum_{m=0}^{M-1}\left(\left[K \sum_{n=0}^{\infty}\left(\Re\left(a_{n}\right) \cos \left(n \theta_{m}\right)-\mathfrak{I}\left(a_{n}\right) \sin \left(n \theta_{m}\right)\right)+\right.\right. \\
\left.\left.K_{j}^{+} \sum_{n=0}^{\infty}\left(\Re\left(a_{n}\right) \cos \left(n \theta_{m}\right)+\mathfrak{I}\left(a_{n}\right) \sin \left(n \theta_{m}\right)\right)-\left(K_{j}^{+}-K\right) \Phi_{\neq j}\left(\theta_{m}\right) e^{-i m \theta_{m}}\right]^{2}\right)=0
\end{array}
$$

Aplicando a derivada,

$$
\begin{array}{r}
\left(\left[K \sum_{n=0}^{\infty} \sum_{m=0}^{M-1}\left(\Re\left(a_{n}\right) \cos \left(n \theta_{m}\right)-\mathfrak{I}\left(a_{n}\right) \sin \left(n \theta_{m}\right)\right)+\right.\right. \\
\left.\left.K_{j}^{+} \sum_{n=0}^{\infty} \sum_{m=0}^{M-1}\left(\Re\left(a_{n}\right) \cos \left(n \theta_{m}\right)+\mathfrak{I}\left(a_{n}\right) \sin \left(n \theta_{m}\right)\right)-\left(K_{j}^{+}-K\right) \Phi_{\neq j}\left(\theta_{m}\right) e^{-i m \theta_{m}}\right]\right)\left(K+k_{j}^{+}\right) \cos \left(k \theta_{m}\right)=0
\end{array}
$$

Se os pontos $\theta_{m}$ forem escolhidos como as frequências de Fourier $\theta_{m}=2 \pi m / M$, a ortogonalidade discreta da série de Fourier pode ser usada, e a equação (6.19) é simplificada:

$$
a_{n}=\frac{1}{M} \frac{k_{j}^{+}-K}{k_{j}^{+}+K} \sum_{m=0}^{M-1} \Phi_{\neq j}\left(\theta_{m}\right) e^{-i m \theta}
$$

para $n>0 \mathrm{e}$

$$
a_{0}=\frac{1}{M} \frac{k_{j}^{+}-K}{K} \sum_{m=0}^{M-1} \Phi_{\neq j}\left(\theta_{m}\right)
$$

para $n=0$. Ao fazer-se o limite de $M \rightarrow \infty$, esta expansão aproxima-se da integral (6.15a) usando uma regra do trapézio. No caso em que $M$ é bem maior que o número de graus de liberdade do elemento, essa aproximação leva a resultados muito próximos ao uso de algoritmos de integração numérica (93), e explicado na seção (7.1.2). 


\subsection{Line Doublets e Inomogeneidades Poligonais}

O potencial complexo, junto com as transformações de coordenadas de $z$ global para a coordenada $\chi$ local para line doublets é descrito na seção (6.4.1) e tem a forma

$$
\Omega_{j}(\chi)=\sum_{n=0}^{\infty} a_{n} F_{j, n}(\chi)
$$

no qual a função $F_{j, n}\left(\chi_{j}\right)$ para campo próximo é definida como

$$
F_{j, n}\left(\chi_{j}\right)=\left(\chi_{j}^{n}+\left(\frac{1}{\chi_{j}}\right)^{n}\right) \ln \left(\frac{\chi_{j}-1}{\chi_{j}+1}\right)+2 \beta_{n}+2 \sum_{k=1}^{n-1}\left[\chi^{k}+\left(\frac{1}{\chi_{j}}\right)^{k}\right]
$$

Para campo distante, $F_{j, n}\left(\chi_{j}\right)$ é definida como

$$
F_{j, n}\left(\chi_{j}\right)=\sum_{k=1}^{\infty} \alpha_{k}\left(\frac{1}{\chi_{j}}\right)^{k}
$$

O coeficiente $\alpha_{j}$ é igual a

$$
\begin{array}{lr}
\alpha_{j}=2\left(\beta_{n-j}-\beta_{n+j}\right), & j=1, \ldots, n-1 \\
\alpha_{j}=-2 \beta_{2 n}, & j=n \\
\alpha_{j}=-2\left(\beta_{j-n}+\beta_{n+j}\right), & j=n+1, \ldots, \infty
\end{array}
$$

e $\beta_{n}$ é definido como

$$
\begin{array}{ll}
\beta_{j}=\frac{1}{j}, & j=1,3,5 \ldots \infty \\
\beta_{j}=0, & j=2,4,6 \ldots \infty
\end{array}
$$

Os coeficientes desconhecidos do potencial complexo gerado por uma line doublet podem ser calculados usando a integral de Cauchy e ortogonalidade de uma expansão em série de Fourier.

\subsubsection{Ortogonalidade da Série de Fourier}

Os coeficientes desconhecidos da expansão do potencial para uma inomogeneidade poligonal modelada por line doubletspodem ser calculados usando o formalismo da ortogonalidade da Série de Fourier. Considerando a expansão em polinômios de Chebyshev no círculo de raio 1 no plano $\chi$,

$$
\lambda(\chi)=\sum_{n=0}^{\infty} a_{n} T_{n} \frac{1}{2}\left(\chi+\frac{1}{\chi}\right)=\sum_{n=0}^{\infty} a_{n}\left(\chi^{n}+\chi^{-n}\right)
$$


e com a integração, dada pela Fórmula de Cauchy, feita no círculo unitário $\chi=e^{-i \theta}$, esta expansão é igual a

$$
\lambda(\theta)=\sum_{n=0}^{\infty} a_{n}\left(e^{i n \theta}+e^{-i n \theta}\right)=2 \sum_{n=0}^{\infty} a_{n} \cos (n \theta)
$$

o que é similar a uma expansão de Fourier. Usando a ortogonalidade de Fourier, os coeficientes $a_{n}$ podem ser calculados. Multiplicando ambos os lados por $\cos (m \theta)$ e integrando no intervalo $[0, \pi]^{2}$

$$
\int_{0}^{\pi} \lambda(\theta) \cos (m \theta) d \theta=2 \int_{0}^{\pi} \sum_{n=0}^{\infty} a_{n} \cos (n \theta) \cos (m \theta) d \theta
$$

A ortogonalidade fornece $\pi \delta_{m n}$ :

$$
2 \sum_{n=0}^{\infty} a_{n} \int_{0}^{\pi} \cos (n \theta) \cos (m \theta) d \theta=2 \pi \sum_{n=0}^{\infty} a_{n} \delta_{m n}
$$

de forma que somente os termos com $m=n$ são diferentes de zero. A expressão (6.29) resulta em

$$
\frac{1}{\pi} \int_{0}^{\pi} \lambda(\theta) \cos (n \theta) d \theta=a_{n}
$$

A expressão final é igual a

$$
a_{n}=\frac{1}{\pi} \int_{0}^{\pi} \lambda(\theta) \cos (n \theta) d \theta
$$

\subsubsection{Integral de Cauchy}

O desenvolvimento geral da seção (6.2.1) é aplicado no caso da line doublet para obter os coeficientes desconhecidos da expansão do potencial para uma inomogeneidade poligonal modelada por line doublets. Dada uma função complexa $H(\chi)=\Xi(\chi)+i \Upsilon(\chi)$, sua parte real é dada por

$$
\Xi(\chi)=\frac{1}{2}(H+\bar{H})
$$

e expandida na série de potências

$$
H=\sum_{n} d_{n} \chi^{n}
$$

na qual a parte real é igual a

$$
\Xi(\chi)=\sum_{n} d_{n} \frac{1}{2}\left(\chi^{n}+\chi^{-n}\right)
$$

\footnotetext{
${ }^{2}$ Para percorrer o elemento apenas uma vez. Ver seção (4.4.1).
} 
usando o fato que $\chi$ percorre o círculo unitário, onde a relação $\bar{\chi}=\chi^{-1}$ e $\chi=e^{i \theta}$ são válidas, com $d_{n}$ real. Usando a equação (6.8), os coeficientes são iguais a

$$
d_{n}=\frac{1}{\pi} \int_{0}^{2 \pi} \Xi(\theta) e^{-i n \theta} d \theta=\frac{1}{\pi} \int_{0}^{\pi} \Xi(\theta) e^{-i n \theta} d \theta+\frac{1}{\pi} \int_{\pi}^{2 \pi=0} \Xi(\theta) e^{-i n \theta} d \theta
$$

A função $\Xi(\theta)$ é assumida como simétrica, portanto $\Xi(-\theta)=\Xi(\theta)$. Mudando $\theta=-\theta$ na segunda integral, obtém-se

$$
d_{n}=\frac{1}{\pi} \int_{0}^{\pi} \Xi(\theta) e^{-i n \theta} d \theta+\frac{1}{\pi} \int_{\pi}^{0} \Xi(-\theta) e^{i n \theta} d \theta
$$

Invertendo os limites de integração na segunda integral e juntando as duas integrais em uma,

$$
\begin{array}{r}
d_{n}=\frac{1}{\pi} \int_{0}^{\pi} \Xi(\theta) e^{-i n \theta} d \theta+\frac{1}{\pi} \int_{0}^{\pi} \Xi(-\theta) e^{i n \theta} d \theta=\frac{1}{\pi} \int_{0}^{\pi}\left(\Xi(\theta) e^{-i n \theta}+\Xi(-\theta) e^{i n \theta}\right) d \theta \\
d_{n}=\frac{1}{\pi} \int_{0}^{\pi}\left(\Xi(\theta) e^{-i n \theta}+\Xi(\theta) e^{i n \theta}\right) d \theta=\frac{1}{\pi} \int_{0}^{\pi} \Xi(\theta)\left(e^{-i n \theta}+e^{i n \theta}\right) d \theta
\end{array}
$$

Usando a identidade de Euler

$$
\left(e^{-i n \theta}+e^{i n \theta}\right)=2 \cos (n \theta)
$$

a integral (6.38) é igual a

$$
d_{n}=\frac{2}{\pi} \int_{0}^{\pi} \Xi(\theta) \cos (n \theta) d \theta
$$

A intensidade de dipolo da line doublet , $\lambda$, é igual a

$$
\lambda(\chi)=\sum a_{n}\left(\chi^{n}+\left(\frac{1}{\chi}\right)^{n}\right)
$$

Ao comparar-se as expressões para a expansão de $\Xi$ e de $\lambda$, nota-se que

$$
\begin{gathered}
\lambda=\sum a_{n}\left(\chi^{n}+\left(\frac{1}{\chi}\right)^{n}\right) \\
\Xi=\sum_{n} d_{n} \frac{1}{2}\left(\chi^{n}+\left(\frac{1}{\chi}\right)^{n}\right)
\end{gathered}
$$

Como as duas funções satisfazem as mesmas condições e estão sendo calculadas na borda do circulo unitário no plano $\chi$, uma relação entre os coeficientes pode ser usada:

$$
a_{n}=\frac{d_{n}}{2}
$$

E portanto

$$
a_{n}=\frac{1}{\pi} \int_{0}^{\pi} \lambda(\theta) \cos (n \theta) d \theta
$$

obtendo a mesma expressão quando a ortogonalidade de Fourier foi utilizada. 


\section{Definição da Descontinuidade no Potencial em Função do Potencial Total}

A intensidade de dipolo do elemento line doublet, igual à descontinuidade do potencial $\lambda$ para a line doublet , pode ser expressa como uma função da carga e das condutividades hidráulicas:

$$
\lambda(Z)=\Phi^{+}(Z)-\Phi^{-}(Z)=\left(K^{+}-K\right) f(\phi(Z))
$$

Para um ponto $Z$ pertence ao polígono formado pelas line doublets, o valor principal é definido como a média do valor do potencial em cada lado do elemento:

$$
\Phi_{p}(Z)=\frac{1}{2}\left(\Phi^{+}+\Phi^{-}\right)=\frac{1}{2}\left(K^{+}+K\right) f(\phi(Z))
$$

e para um ponto $Z$ fora do polígono,

$$
\Phi_{p}(Z)=\frac{1}{2}(K+K) f(\phi(Z))=K f(\phi(Z))
$$

Uma relação entre a descontinuidade de potencial e o valor principal pode ser estabelecida:

$$
\lambda=2 \frac{\left(K^{+}-K\right)}{\left(K^{+}+K\right)} \Phi_{p}
$$

obtendo uma relação entre a descontinuidade do potencial $\lambda$ e o potencial geral $\Phi_{p}$. Outra forma de se obter a mesma relação sem usar o valor principal é descrita abaixo. A condição de contorno para uma inomogeneidade poligonal é

$$
\frac{\Phi_{T}^{+}}{k_{j}^{+}}=\frac{\Phi_{T}^{-}}{K}
$$

O potencial total $T$ pode ser dividido em

$$
\Phi_{T}=\Phi_{\neq j}+\Phi_{j}
$$

no qual o índice $j$ denota o elemento analisado em específico. O potencial só é descontínuo para o elemento $j$, enquanto o potencial de todos os outros elementos é contínuo através da inomogeneidade, como visto na condição de contorno (5.3.1), ou seja, $\Phi_{\neq}^{+}=\Phi_{\neq}^{-}$:

$$
\Phi_{T}^{+}-\Phi_{T}^{-}=\Phi_{\neq j}-\Phi_{\neq j}+\Phi_{j}^{+}-\Phi_{j}^{-}=\Phi_{j}^{+}-\Phi_{j}^{-}
$$

e a descontinuidade do potencial, gerado pela intensidade de dipolo $\lambda$, é igual a

$$
\lambda=\Phi_{T}^{+}-\Phi_{T}^{-}
$$

Usando a equação (6.51), o valor para $\lambda$ pode ser reescrito como

$$
\lambda=\frac{K_{j}^{+}-K}{K} \Phi_{T}^{-}
$$


A descontinuidade do potencial, portanto, é igual a

$$
\Phi_{T}^{+}-\Phi_{T}^{-}=\frac{K_{j}^{+}-K}{K} \Phi_{T}^{-}=\frac{K_{j}^{+}-K}{K}\left(\Phi_{\neq j}+\Phi_{j}^{-}\right)
$$

Notando que

$$
\Phi_{j}^{-}=-\frac{1}{2}\left(\Phi_{j}^{+}+\Phi_{j}^{-}+\Phi_{\neq j}^{+}-\Phi_{\neq j}^{-}\right)=-\frac{1}{2}\left(\Phi_{T}^{+}-\Phi_{T}^{-}\right)
$$

e substituindo na equação (6.56),

$$
\Phi_{T}^{+}-\Phi_{T}^{-}=\frac{K_{j}^{+}-K}{K}\left(\Phi_{\neq j}-\frac{1}{2}\left(\Phi_{T}^{+}-\Phi_{T}^{-}\right)\right)
$$

só é necessário inverter os termos, para obter

$$
\lambda=\Phi_{T}^{+}-\Phi_{T}^{-}=2\left(\frac{K_{j}^{+}-K}{K_{j}^{+}+K}\right) \Phi_{\neq j}
$$

A descontinuidade do potencial $\lambda$ pode ser substituído na equação (6.46), obtendo-se uma expressão para se encontrar os coeficientes $a_{n}$ da line doublet $i$ em função do valor do potencial causado por todos os outros elementos presentes no sistema:

$$
a_{i n}=\frac{\left(K_{i}^{+}-K\right)}{\left(K_{i}^{+}+K\right)} \frac{1}{2 \pi} \int_{0}^{\pi} \Phi_{\neq j}\left(\theta_{i}\right) \cos \left(n \theta_{i}\right) d \theta_{i}
$$

\subsubsection{Mínimos Quadrados}

A abordagem de mínimos quadrados para as line doublets é similar à usada para as inomogeneidades circulares. Com o sistema descrito por (6.60), as expressões para integração são substituídas pelas expressões para mínimos quadrados, mantendo o termo da expansão cosseno e a função a ser calculada para se avaliar a condição de contorno (6.4.1). A condição de contorno a ser minimizada para o caso da line doublet é semelhante à do caso para a inomogeneidade circular:

$$
\sum_{m=0}^{M-1}\left[K \Phi_{j}^{+}-k_{j} \Phi_{j}^{-}-\left(k_{j}-k\right) \Phi_{\neq j}\right]^{2} \rightarrow \operatorname{MIN}\left[a_{0}, \ldots, a_{n}\right]
$$

Seguindo o mesmo procedimento descrito na seção (6.3.2), obtém-se a expressão semelhante. A equivalência entre o método de mínimos quadrados e a integração numérica é explicada na seção (6.7).

\subsection{Fraturas}

Esta seção descreve uma das principais contribuições originais deste trabalho, descrevendo o uso das condições de contorno para o cálculo dos coeficientes desconhecidos para o elemento analítico 
para fraturas. As condições de contorno para a fratura, vistas na seção (5.4.1), são aplicadas para encontrar-se uma expressão para calcular os coeficientes desconhecidos da expansão em série para o potencial complexo do elemento para uma fratura. A abordagem via ortogonalidade da série de Fourier e a integral de Cauchy também são aplicadas neste caso.

\subsubsection{Ortogonalidade via Série de Fourier}

Da mesma forma do que para o inomogeneidade circular e para a poligonal, a ortogonalidade pode ser usada. A descontinuidade do potencial é dada como a parte imaginária, a função de corrente $\Psi$, portanto é igual a

$$
\mu=-\mathfrak{I}\left(\sum a_{n}\left(\chi^{-n}-\chi^{n}\right)\right)=-2 \sum a_{n} \sin (n \theta)
$$

o que é igual a uma série de Fourier em senos. O intervalo a ser usado para a aplicação da ortogonalidade é de $[-1,1]$, sobre o elemento, corresponde a $[\pi, 0]$ no plano $\chi$. Aplicando a ortogonalidade,

$$
\begin{array}{r}
-2 a_{n}=\frac{2}{\pi} \int_{0}^{\pi} \mu(\theta) \sin (n \theta) d \theta \\
a_{n}=-\frac{1}{\pi} \int_{0}^{\pi} \mu(\theta) \sin (n \theta) d \theta
\end{array}
$$

que resulta na mesma expressão do caso de Cauchy, descrita na seção (6.5.2).

\subsubsection{Integral de Cauchy}

A parte imaginária de $H, \Upsilon$, similarmente ao que foi feito para as line doublets para a parte real, pode ser descrita por

$$
\Upsilon=\frac{1}{2}(H-\bar{H})=\sum_{n} d_{n} \frac{1}{2}\left(\chi^{n}-\chi^{-n}\right)
$$

Considera-se $\chi$ percorrendo o círculo unitário, onde a relação $\bar{\chi}=\chi^{-1}$ vale, com $d_{n}$ reais. Os coeficientes são iguais a

$$
d_{n}=\frac{i}{\pi} \int_{0}^{2 \pi} \Upsilon(\theta) e^{-i n \theta} d \theta=\frac{i}{\pi} \int_{0}^{\pi} \Upsilon(\theta) e^{-i n \theta} d \theta+\frac{i}{\pi} \int_{\pi}^{2 \pi=0} \Upsilon(\theta) e^{-i n \theta} d \theta
$$

A função $\Upsilon(\theta)$ é antissimétrica, portanto $\Upsilon(-\theta)=-\Upsilon(\theta)$. Mudando $\theta=-\theta$ na segunda integral,

$$
d_{n}=\frac{i}{\pi} \int_{0}^{\pi} \Upsilon(\theta) e^{-i n \theta} d \theta-\frac{i}{\pi} \int_{\pi}^{0} \Upsilon(-\theta) e^{i n \theta} d \theta
$$


Invertendo os limites de integração da segunda integral e colapsando as duas integrais em uma,

$$
\begin{array}{r}
d_{n}=\frac{i}{\pi} \int_{0}^{\pi} \Upsilon(\theta) e^{-i n \theta} d \theta+\frac{i}{\pi} \int_{0}^{\pi} \Upsilon(-\theta) e^{i n \theta} d \theta=\frac{i}{\pi} \int_{0}^{\pi}\left(\Upsilon(\theta) e^{-i n \theta}+\Upsilon(-\theta) e^{i n \theta}\right) d \theta \\
d_{n}=\frac{i}{\pi} \int_{0}^{\pi}\left(\Upsilon(\theta) e^{-i n \theta}-\Upsilon(\theta) e^{i n \theta}\right) d \theta=\frac{i}{\pi} \int_{0}^{\pi} \Upsilon(\theta)\left(e^{-i n \theta}-e^{i n \theta}\right) d \theta
\end{array}
$$

Com a identidade de Euler,

$$
\left(e^{-i n \theta}-e^{i n \theta}\right)=-2 i \sin (n \theta)
$$

A integral acima é igual a

$$
d_{n}=(-2 i) \frac{i}{\pi} \int_{0}^{\pi} \Upsilon(\theta) \sin (n \theta) d \theta=\frac{2}{\pi} \int_{0}^{\pi} \Upsilon(\theta) \sin (n \theta) d \theta
$$

Novamente, devido às condições de solução de ambos, os coeficientes de $\mu$ e $\Upsilon$ podem ser comparados

$$
\begin{array}{r}
\mu=\sum a_{n}\left(\chi^{-n}-\chi^{n}\right) \\
\Upsilon=\sum_{n} d_{n} \frac{1}{2}\left(\chi^{n}-\chi^{-n}\right)
\end{array}
$$

Sendo que a seguinte relação pode ser estabelecida:

$$
-a_{n}=\frac{d_{n}}{2}
$$

A equação para os coeficientes então é igual a

$$
\begin{aligned}
2 a_{n} & =-\frac{2}{\pi} \int_{0}^{\pi} \mu(\theta) \sin (n \theta) d \theta \\
a_{n} & =-\frac{1}{\pi} \int_{0}^{\pi} \mu(\theta) \sin (n \theta) d \theta
\end{aligned}
$$

A mesma expressão obtida ao se usar a ortogonalidade de Fourier. A descontinuidade do potencial $\mu$, por representar a descontinuidade do potencial no contorno da fratura, pode ser substituído pela equação (5.58):

$$
a_{i, n}=-\frac{1}{\pi} \frac{K_{i}^{+} b_{i}^{*}}{K} \int_{0}^{\pi} \Re\left(W_{T}\left(\theta_{i}\right) e^{i \alpha_{i}}\right) \sin \left(\theta_{i}\right) \sin \left(n \theta_{i}\right) d \theta_{i}
$$

onde $W_{T}$ é a soma das descargas de todos os elementos.

\subsection{Algoritmos de Solução}

Os algoritmos apresentados nesta seção foram usados para encontrar os coeficientes desconhecidos dos elementos analíticos, com variados graus de sucesso. Os algoritmos de solução iterativos, tanto o explícito quando o implícito, necessitam de um teste de convergência, que é descrito na 
seção (6.9.1). O capítulo (7) também apresenta a análise de erro usada para comparar os diferentes algoritmos de solução.

\subsubsection{Algoritmo de Solução Iterativo Implícito}

Janković e Barnes $(85,93)$ propuseram um algoritmo de solução das equações (6.77), (6.60) e (6.15a) baseado no algoritmo de Gauss-Seidel (99), mas sem construir explicitamente a matriz do sistema linear. Strack (48) também usa esse método para calcular os coeficientes. O método consiste em assumir que o lado direito de (6.77), (6.60) e (6.15a) é conhecido e fixo, enquanto se calcula o coeficiente $a_{i n}$. O coeficiente atualizado é usado para o cálculo dos coeficientes do próximo elemento. Esse processo continua até a variação dos coeficientes entre iterações ser menor que um determinado valor especificado. As integrais podem ser transformadas em somas discretas, o que é similar a resolver o sistema de mínimos quadrados, ou resolvidas de forma numérica.

Como exemplo, considere-se um sistema com uma inomogeneidade poligonal composta por $M$ line doublets formando um polígono fechado, e um campo de escoamento uniforme. A expressão para calcular o coeficiente da line doublet $i$ de ordem $k$ é igual a

$$
a_{i, k}=P_{i, k} \int_{0}^{\pi} \Re\left(\Omega_{T}\left(\theta_{i}\right)\right) \cos \left(k \theta_{i}\right) d \theta_{i}
$$

ou, de forma explícita,

$$
a_{i, k}=P_{i, k} \int_{0}^{\pi} \Re\left(\sum_{j, m} a_{j, m} F_{j, m}\left(\theta_{i}\right)\right) \cos \left(k \theta_{i}\right) d \theta_{i}+P_{i, k} \int_{0}^{\pi} \Re\left(\Omega_{u f}\left(\theta_{i}\right)\right) \cos \left(k \theta_{i}\right) d \theta_{i}
$$

onde

$$
P_{i, k}= \begin{cases}\frac{2}{\pi}\left(\frac{K^{+}-K}{K^{+}+K}\right), & n=0 \\ \frac{4}{\pi}\left(\frac{K^{+}-K}{K^{+}+K}\right), & n>0\end{cases}
$$

Como mencionado acima, os termos do lado direito são considerados conhecidos da iteração anterior, e mantidos constantes enquanto os coeficientes para o elemento $i$ são calculados. A integral pode ser trocada de ordem com a soma, obtendo-se

$$
a_{i, n}^{p+1}=P_{i, n} \sum_{j, m} a_{j, m}^{p} \int_{0}^{\pi} \Re\left(F_{j, m}\left(\theta_{i}\right)\right) \cos \left(n \theta_{i}\right) d \theta_{i}+P_{i, n} \int_{0}^{\pi} \Re\left(\Omega_{u f}\left(\theta_{i}\right)\right) \cos \left(n \theta_{i}\right) d \theta_{i}
$$

com

$$
\Omega_{u f}(z)=-Q_{0} z e^{-i \gamma}+C
$$

onde o índice $p$ indica o contador do processo iterativo. Os coeficientes $a_{j, m}^{0}$ são iniciados em zero. Esse método é descrito em $(85,92)$, e desenvolvido no plano $\chi$ para séries de Chebyshev em (48). O mesmo processo pode ser aplicado às inomogeneidades circulares. A forma iterativa de calcular os 
coeficientes para as inomogeneidades circulares é dada por

$$
a_{i, n}=P_{i, n} \int_{0}^{2 \pi} \Re\left(\Omega_{j}\left(\theta_{i}\right)\right) e^{-i n \theta_{i}} d \theta_{i}+P_{i, n} \int_{0}^{\pi} \Re\left(\Omega_{u f}\left(\theta_{i}\right)\right) e^{-i n \theta_{i}} d \theta_{i}
$$

onde o potencial para o escoamento uniforme é o mesmo dado por (6.82). De forma explícita,

$$
\left.a_{i, n}^{k+1}=P_{i, n} \sum_{j, m} a_{j, m}^{k} \int_{0}^{2 \pi} \Re\left(Z_{j}^{-m}\right)\right) e^{-i n \theta_{i}} d \theta_{i}+P_{i, n} \int_{0}^{\pi} \Re\left(\Omega_{u f}\left(\theta_{i}\right)\right) e^{-i n \theta_{i}} d \theta_{i}
$$

Para as fraturas, o procedimento é o mesmo: o potencial complexo é dado por

$$
\Omega_{i}\left(Z_{i}\right)=\sum_{n=1}^{\infty} a_{i, n}\left(Z_{i}-\sqrt{Z_{i}-1} \sqrt{Z_{i}+1}\right)^{n}
$$

com os coeficientes $a_{i, n}$ reais. O método iterativo de solução proposto é igual a

$$
a_{i, n}=P_{i, n} \int_{0}^{\pi} \Re\left(W\left(\theta_{i}\right) e^{i \alpha_{i}}\right) \sin \left(\theta_{i}\right) \sin \left(n \theta_{i}\right) \theta_{i}-P_{i, n} \int_{0}^{\pi} \Re\left(\frac{d \Omega_{u f}\left(\theta_{i}\right)}{d z} e^{i \alpha_{i}}\right) \sin \left(\theta_{i}\right) \sin \left(n \theta_{i}\right) d \theta_{i}
$$

no qual $W$ é o vetor descarga, definido em 5.4.1, dado por

$$
\begin{array}{r}
W(z, i)=-\frac{d \Omega_{i}(z)}{d z} \\
W\left(Z_{i}\right)=-\frac{d \Omega_{i}\left(Z_{i}\right)}{d Z_{i}} \frac{d Z_{i}}{d z}
\end{array}
$$

A expressão explícita é igual a

$$
a_{i, n}^{k+1}=P_{i, n} \sum_{j, m}^{M, N} a_{j, m}^{k} \int_{0}^{\pi} \Re\left(\frac{\left(Z_{j}-\sqrt{Z_{j}-1} \sqrt{Z_{j}+1}\right)^{m}}{d Z_{j}} \frac{d Z_{j}}{d z} e^{i \alpha_{i}}\right) \sin \left(\theta_{i}\right) \sin \left(n \theta_{i}\right) d \theta_{i}
$$

sendo iterada por todas as fraturas até a convergência.

\section{Procedimento de Cálculo para o Método Iterativo Implícito}

Um elemento é calculado considerando que todos os outros elementos são mantidos fixos. $\mathrm{O}$ processo pode ser explicado com um loop interno e um externo.

Loop interno:

1. Para o elemento $j$, coeficiente de ordem $\mathrm{m}\left(a_{j, m}\right)$ :

(a) Somar a contribuição de todos os outros elementos (potencial ou descarga)

(b) Somar a contribuição do campo de escoamento uniforme

(c) Integrar a soma acima no sistema de coordenadas local do elemento $j$ 
(d) atribuir a $a_{j, m}$ o novo coeficiente calculado

Loop externo:

1. Para todos os elementos, para todos os coeficientes $n_{\max }$ :

(a) Executar loop interno

(b) Recalcular a constante

(c) Verificar a convergência

O cálculo da constante é sempre feito de acordo com o potencial total disponível naquela iteração. A constante é igual ao valor do potencial total, $\Omega$, que é a soma dos potenciais $\Omega_{j}$ de todos os elementos analíticos presentes no domínio, menos o valor de potencial no ponto de referência, $\Phi_{0}$ :

$$
C=\sum_{j=1}^{N} \Omega_{j}\left(z_{r e f}\right)-\Phi_{0}
$$

\subsubsection{Algoritmo de Solução Matricial}

Este método de solução, juntamente com o elemento analítico para várias fraturas, consistem nos principais avanços deste trabalho. O método matricial apresentado nesta seção surge de uma dificuldade particular com o elemento analítico para fratura, e foi estendido para todos os elementos, servindo como uma abordagem de solução geral dentro do MEA.

O método original de solução do MEA, apresentado em (1), envolvia a construção da matriz de colocação, onde o potencial de cada elemento era avaliado nos pontos de colocação, mais o valor do potencial de cada elemento em um ponto de referência com carga conhecida. O sistema linear formado,

$$
A x=c
$$

era resolvido usando técnicas de solução de sistemas lineares. O desenvolvimento do método de solução iterativo implícito permitiu um aumento na precisão nas soluções do MEA com uma diminuição do custo computacional $(85,92,93)$. Apesar dos resultados satisfatórios de tal método, (85) reporta casos nos quais a convergência não foi encontrada, considerando que a soma envolvida para todos os elementos e a subtração de números próximos à capacidade de representação de ponto flutuante poderia levar a instabilidade no algoritmo de solução e à divergência.

Ao desenvolver o elemento para fratura, a abordagem inicial foi usar o algoritmo iterativo implícito. Mas após exaustivos testes, a maioria dos casos mostraram-se divergentes. Tentativas de mitigar o problema, como a separação da soma de cada elemento, não tiveram sucesso. Para tentar sanar esse problema foi feita uma volta à um método matricial. Cada elemento da matriz é composto por uma integral sobre o potencial para aquele ponto para a ordem do elemento analítico. Esse método matricial é capaz de fornecer soluções para o caso de fraturas, e pode ser aplicado 
às inomogeneidades circulares e poligonais. A comparação com as soluções iterativas implícitas, matricial e explícitas apresentam também possibilidades de melhoria.

O custo computacional em tempo de processamento e memória é um dos fatores a ser considerado, já que o método matricial necessita que a matriz seja construída completamente e envolve pelo menos uma inversão de matriz, o que pode ser custoso.

A construção da matriz permite que a estrutura da mesma seja analisada com respeito a norma e raio espectral, fornecendo mais informações sobre a natureza numérica do problema e suas limitações. A formulação matricial ainda permite que algoritmos de solução iterativos possam ser usados, como Jacobi e Gauss-Seidel, e facilita avanços futuros como técnicas de solução paralelas.

Além disso, pacotes computacionais como o Scipy (98) e o Lapack (100) apresentam rotinas de solução direta e iterativa de sistemas lineares. Tais rotinas foram testadas pelas mais diferentes comunidades científicas, além de serem software livre.

Como cada elemento $j$ é composto por $m$ coeficientes, a notação desta seção reflete tal comportamento: o índice composto $a_{j, m}$ é referente ao coeficiente $m$ da inomogeneidade $j$.

\subsubsection{Forma Matricial para Inomogeneidade Circular}

A forma matricial é construída a partir da expressão para o cálculo dos coeficientes desconhecidos para o potencial da inomogeneidade circular. O vetor conhecido do sistema linear é dado pela equação (6.92), que consiste na integração do escoamento uniforme:

$$
c_{i, n}=\int_{0}^{2 \pi} \Re\left(Q_{0} z e^{-i \gamma}\right) e^{-i n \theta_{i}} d \theta_{i}
$$

onde $c_{i, n}$ representa o elemento $(i, n)$ do vetor conhecido $c$. O coeficiente $a_{i, n}$, quando passado para o lado direito da equação, leva ao aparecimento de uma delta de Dirac em $j$ e em $m$, já que tal termo só é presente na diagonal da matriz:

$$
\left.\int_{0}^{2 \pi} \Re\left(Q_{0} z e^{-i \gamma}\right) e^{-i n \theta_{i}} d \theta_{i}=\sum_{j, m}^{M, N} a_{j, m}\left(\int_{0}^{\pi} \Re\left(Z_{j}^{-m}\right)\right)\left(\theta_{i}\right) e^{-i n \theta_{i}} d \theta_{i}\right)-\frac{a_{i, n}}{P_{i, n}}+\int_{0}^{\pi} C \cos \left(n \theta_{i}\right) d \theta_{i}
$$

Todos os coeficientes $a_{j, m}$ são desconhecidos agora, e a função delta de Dirac é introduzida:

$$
\left.\int_{0}^{2 \pi} \Re\left(Q_{0} z e^{-i \gamma}\right) e^{-i n \theta_{i}} d \theta_{i}=\sum_{j, m}^{M, N} a_{j, m}\left(\int_{0}^{\pi} \Re\left(Z_{j}^{-m}\right)\right)\left(\theta_{i}\right) e^{-i n \theta_{i}} d \theta_{i}-\frac{\delta_{i, j} \delta_{m, n}}{P_{i, n}}\right)+\int_{0}^{\pi} C \cos \left(n \theta_{i}\right) d \theta_{i}
$$


na qual os coeficientes $P_{i, n}$ são iguais a

$$
P_{i, n}= \begin{cases}\frac{2}{\pi}\left(\frac{k_{\infty}}{K^{+}+k_{\infty}}\right), & n=0 \\ \frac{1}{\pi}\left(\frac{K^{+}-k_{\infty}}{K^{+}+k_{\infty}}\right), & n>0\end{cases}
$$

A condição de contorno para a inomogeneidade circular (5.2.1) mostra que o coeficiente da matriz de auto-interação é igual a $P_{i, n}$, já que a auto-interação do elemento analítico é zero.

\subsubsection{Forma Matricial para Inomogeneidade Poligonal}

Da mesma forma que para a inomogeneidade circular, o sistema iterativo implícito é usado como base para a forma matricial, e a equação (6.60) é transformada para uma forma matricial, no qual novamente o elemento diagonal contém o termo $P_{i, n}$

$$
\int_{0}^{\pi} \Re\left(Q_{0} z e^{-i \gamma}\right) \cos \left(n \theta_{i}\right) d \theta_{i}=\sum_{j, m}^{M, N} a_{j, m}\left(\int_{0}^{\pi} F_{j, m}\left(\theta_{i}\right) \cos \left(n \theta_{i}\right) d \theta_{i}-\frac{\delta_{i, j} \delta_{m, n}}{P_{i, n}}\right)+\int_{0}^{\pi} C \cos \left(n \theta_{i}\right) d \theta_{i}
$$

na qual $M$ é o número de elementos presentes e $N$ a ordem da expansão. O lado esquerdo da expressão (6.96) é o vetor conhecido $b$; os coeficientes $a_{j, m}$ formam o vetor de incógnitas $x$, enquanto a expressão entre parêntesis forma a matriz $A$. Nota-se que o índice $(j, m)$ é composto: cada elemento $j$ é formado pela expansão em $m$ termos. A última coluna e a última linha da matriz são provenientes do cálculo da constante no ponto de referência. Como no caso da inomogeneidade circular, o termo de auto-interação é sempre zero, como indicam as condições de contorno 6.4.1:

$$
\int_{0}^{\pi} F_{i, n} \cos \left(n \theta_{i}\right) d \theta_{i}=0
$$

\subsubsection{Forma Matricial para Fratura}

Repetindo o procedimento, a partir da forma iterativa, a expressão para a forma matricial para fraturas é obtida:

$$
\begin{array}{r}
\int_{0}^{\pi} \Re\left(Q_{0} e^{-i(\gamma+\alpha)}\right) \sin \left(\theta_{i}\right) \sin n \theta_{i} d \theta_{i}= \\
\sum_{j, m}^{M, N} a_{j, m}\left(\int_{0}^{\pi} \Re\left(\frac{\left(Z_{j}-\sqrt{Z_{j}-1} \sqrt{Z_{j}+1}\right)^{m}}{d Z_{j}} \frac{d Z_{j}}{d z} e^{i \alpha_{i}}\right)\left(\theta_{i}\right) \sin \left(\theta_{i}\right) \sin n \theta_{i} d \theta_{i}-\frac{\delta_{i, j} \delta_{m, n}}{P_{i, n}}\right)+ \\
\int_{0}^{\pi} C \sin \left(\theta_{i}\right) \sin n \theta_{i} d \theta_{i}
\end{array}
$$


na qual $P_{i, n}$ é igual a

$$
P_{i, n}=\left\{\frac{1}{\pi}\left(\frac{K^{+} b}{K}\right), \quad \forall n\right.
$$

Diferentemente do caso das inomogeneidades circulares e das line doublets, a auto-interação das fraturas não é nula.

\section{Forma Geral da Solução Matricial}

A forma matricial para os três casos é semelhante, mudando apenas o coeficiente $P_{i, n}$, o termo de ortogonalidade na integração e o termo de potencial. De forma geral, o problema matricial para os três casos pode ser resumido na expressão

$$
\int_{a}^{b} G_{u f} U_{i, n} d U_{i, n}=\sum_{j, m} a_{j, m}\left(\int_{a}^{b} H_{j, m} U_{i, n} d U_{i, n}-\frac{\delta_{i, j} \delta_{m, n}}{P_{i, n}}\right)+\int_{a}^{b} C U_{i, n} d U_{i, n}
$$

lembrando que o último termo do lado esquerdo, $\Phi_{0}$, é igual ao valor do potencial no ponto de referência. O termo da matriz $A_{i, j}$ é igual a

$$
A_{i, j}=\sum_{j, m}^{M, N}\left(\int_{a}^{b} H_{j, m} U_{i, n} d U_{i, n}-\frac{\delta_{i, j} \delta_{m, n}}{P_{i, n}}\right)
$$

\subsubsection{Forma Geral das Integrais}

As integrais de interação (6.15a), (6.60) e (6.77) podem apresentar formas difíceis de serem resolvidas de forma analítica. As integrais descritas nas seções anteriores envolvem transformações de coordenadas e as características geométricas dos elementos do sistema. A título de exemplo, duas integrais são demonstradas para casos simples de interação entre duas fraturas e para o caso de duas line doublets.

Sejam duas linhas com coordenadas globais $\left(z_{1}^{1}, z_{2}^{1}\right)$ para a linha 1 e $\left(z_{1}^{2}, z_{2}^{2}\right)$ para a linha 2 . Considerando que a integral a ser desenvolvida representa a influência da linha 2 na linha 1 , as seguintes constantes serão úteis na simplificação da integral:

$$
\begin{array}{r}
a=z_{1}^{1}+z_{2}^{1}-z_{1}^{2}-z_{2}^{2} \\
f=z_{2}^{2}-z_{1}^{2} \\
c=z_{1}^{1}+z_{2}^{1}-2 z_{2}^{2} \\
d=z_{1}^{1}+z_{2}^{1}-2 z_{1}^{2} \\
e=z_{1}^{2}+z_{2}^{2}
\end{array}
$$




\section{Duas line doublets}

Com as definições (6.102a), e usando as transformações de coordenadas definidas em 4.4.1, a integral é igual a

$$
\begin{array}{r}
\int_{0}^{\pi} \frac{1}{f^{n}}\left((e \cos (\theta)+\sqrt{e \cos (\theta)+c} \sqrt{e \cos (\theta)+d})^{n}+(e \cos (\theta)-\sqrt{e \cos (\theta)+c} \sqrt{e \cos (\theta)+d})^{n}\right) \\
\ln \left(\frac{e \cos (\theta)+\sqrt{e \cos (\theta)+c} \sqrt{e \cos (\theta)+d}+c}{e \cos (\theta)+\sqrt{e \cos (\theta)+c} \sqrt{e \cos (\theta)+d}+d}\right) \cos (n \theta) d \theta
\end{array}
$$

\section{Duas Fraturas}

Usando as mesmas definições (6.102a), a integral (6.77) para duas fraturas é igual a

$$
\int_{0}^{\pi} \frac{1}{f^{n-1}}\left(\frac{e \cos (\theta)+a-\sqrt{e \cos (\theta)+c} \sqrt{e \cos (\theta)+d}}{\sqrt{e \cos (\theta)+c} \sqrt{e \cos (\theta)+d}}\right)^{n} e^{i \alpha_{i}} \frac{2}{z_{2}^{2}-z_{1}^{2}} \sin (\theta) \sin (n \theta) d \theta
$$

na qual o termo $\frac{2}{z_{2}^{2}-z_{1}^{2}}$ é a derivada $\frac{d Z_{j}}{d z}$.

\subsubsection{Algoritmo de Solução Iterativo Explícito e Partição da Matriz}

O método iterativo implícito não necessita da construção explícita da matriz. Entretanto, uma vez que a matriz é obtida, métodos iterativos de solução do sistema linear $A x=b$ podem ser aplicados, e as matrizes resultantes analisadas quanto à convergência e erro na iteração (99). Em sistemas nos quais tanto um método iterativo quanto um método matricial direto pode ser usado, o método matricial direto deve ser preferido por ser mais estável numericamente, apesar de existir casos onde apenas métodos iterativos são aplicáveis, como casos onde a matriz não cabe na memória principal ${ }^{3}$.

Os métodos de solução de sistemas lineares se baseiam em um particionamento de $A$ para encontrar a solução do sistema com poucas iterações (99).

$$
A=R-T
$$

Com esta partição, o sistema linear pode ser resolvido da seguinte forma: aplicando o particionamento no sistema linear

$$
\begin{array}{r}
A x=b \\
(R-T) x=b \\
R x=T x+b \\
x^{k+1}=R^{-1} T x^{k}+R^{-1} b
\end{array}
$$

\footnotetext{
${ }^{3}$ Prof. José Alberto Cuminato, comunicação pessoal.
} 
O raio espectral da matriz $R^{-1} T$ indica possibilidade de convergência do esquema iterativo de solução. Se o raio espectral, definido como (99)

$$
\rho(G)=\max \left(\left|\lambda_{i}\right|\right)
$$

no qual $\lambda_{i}$ são os autovalores de $G$, for menor que 1 , a solução iterativa converge, para qualquer vetor inicial $x^{0}$ (99).

Duas partições da matriz são aplicadas: a de Jacobi e a de Gauss-Seidel $(94,99)$. Considerando que uma matriz $A$ pode ser separada em três componentes,

$$
A=L+D+U
$$

na qual $L$ é a matriz triangular inferior sem incluir a diagonal, $U$ a matriz triangular superior sem incluir a diagonal, e $D$ a matriz diagonal. A partição da matriz de Jacobi (99) é dada por

$$
\begin{array}{r}
R=D \\
T=-(L+U)
\end{array}
$$

e a partição de Gauss-Seidel (99) é dada por

$$
\begin{array}{r}
R=D+L \\
T=-U
\end{array}
$$

A convergência do método iterativo é dada por

$$
\rho\left(R^{-1} T\right)=\max \left(\left|\lambda_{i}\right|\right)
$$

sendo garantida se $\rho\left(R^{-1} T N\right)<1$ para qualquer vetor inicial $x^{k=0}$. O sistema (6.106a) também foi aplicado para os três casos de estudo. Analisando o algoritmo iterativo implícito, verifica-se que ele tem forma semelhante a um algoritmo de Gauss-Seidel, apesar de apresentar diferenças significativas no que tange a convergência.

\subsection{Integração Numérica versus Mínimos Quadrados}

A abordagem para mínimos quadrados descrita em (85) e (37) consiste em gerar uma matriz retangular e resolver o sistema linear para encontrar os coeficientes desconhecidos da expansão em série dos elementos. Entretanto, é possível montar uma matriz quadrada, em que cada termo da matriz é uma soma dos termos dada por exemplo pela equação (6.3.2). Esta equivalência é explicada abaixo.

O artigo (93) demonstra que a abordagem de mínimos quadrados é equivalente à integração numérica usando o algoritmo do trapézio. A expressão para integração pela regra de trapézio (93) é 
dada por

$$
\begin{array}{r}
\int_{a}^{b} f(x) d x=\frac{1}{2 M} \sum_{n=0}^{M} f\left(x_{i}\right) \\
x_{i}=i *(b-a) / M+a
\end{array}
$$

onde se nota a semelhança com a aproximação de mínimos quadrados. Esta equivalência é encontrada para a line doublet e para a fratura. Substituindo a integral contínua nas equações por uma aproximação da integral pela regra do trapézio, obtém-se a expressão dada por mínimos quadrados. Dessa forma, a integração numérica pode ser efetuada usando algoritmos específicos de integração ou a regra do trapézio. Essa abordagem é válida tanto para calcular os elementos de matriz no método matricial e no método iterativo explícito, quanto para o método iterativo implícito.

\subsection{Linguagens de Programação e Implementação do Có- digo}

A escolha da linguagem de programação e em menor extensão a plataforma de desenvolvimento, especialmente para software científico, leva em conta fatores como disponibilidade das ferramentas, custo, experiência do programador com tais ferramentas e a capacidade da linguagem em satisfazer os requerimentos dos algoritmos.

Batista (51) utilizou a linguagem Python (101) em seu trabalho, utilizando-se de orientação a objeto. Alencar Neto (55) utilizou Java, e fez em seu trabalho uma extensa revisão sobre programação orientada a objetos, e sua adequação na implementação de algoritmos para o MEA. Bakker e Kelson (102) apresentaram uma estratégia para implementação do MEA em Python também usando programação orientada a objetos. Dentre as justificativas dadas por (39) para o uso de Ptyhon, então a facilidade de desenvolvimento de um protótipo e a disponibilidade de bibliotecas são destacadas, apesar de considerar que seu tempo de execução é geralmente maior que de uma linguagem compilada, como Fortran.

Grande parte dos algoritmos deste trabalho foram inicialmente implementados em $\mathrm{C}++$. $\mathrm{C}++$ se apresentou em um primeiro momento como um ponto de equilíbrio entre a velocidade de linguagens compiladas, como Fortran, com a flexibilidade de linguagens interpretadas como Python. Durante o desenvolvimento do trabalho, entretanto, limitações de debugging, tempo de desenvolvimento e a restrição da orientação a objetos do $\mathrm{C}++$ tornaram o desenvolvimento do protótipo muito mais lenta, e o ganho em velocidade de processamento, não sendo um requerimento central nesta fase, não era suficiente para justificar o seu uso. A facilidade com que a prototipagem de um modelo pode ser feita em Python, mais a flexibilidade de uso de diferentes plataformas, e a extensa coleção de bibliotecas científicas disponíveis como software livre tornaram-se decisivas no uso de Python para a implementação dos algoritmos deste trabalho. Os códigos já desenvolvidos em $\mathrm{C}++$ foram transformados para Python, e todos os novos desenvolvimentos forma feitos diretamente em Python. As bibliotecas Scipy (98) e Matplotlib (103), disponíveis como software de código livre, foram usadas 
em diversos pontos do código, como para integração numérica e geração de gráficos.

\subsection{Análise de Erro}

A modelagem numérica de um fenômeno físico, como o escoamento de água subterrânea, consiste na criação de ferramentas computacionais necessárias para a solução das equações governantes que descrevem o modelo físico ou matemático de tal fenômeno (37). A implementação do modelo numérico é geralmente feita em um computador. Computadores, devido a sua própria natureza, apresentam a limitação de apenas poderem trabalhar com representações discretas e finitas de grandezas contínuas, ao contrário de modelos matemáticos e físicos, que podem incluir grandezas contínuas e discretas. Esta limitação é uma das origens do erro associado à modelos numéricos, o erro de arredondamento (94). Outra fonte de erro é o erro de truncamento, associado ao modelo numérico em si e independente do equipamento onde tal algoritmo foi implementado (94). O erro de truncamento surge da quantidade finita de memória de um computador, do uso de séries finitas e da necessidade de se computar de forma discreta quantidades de variáveis contínuas. Um exemplo de erro de truncamento é o erro que surge ao se calcular uma integral de forma numérica, computando a função a ser integrada em um conjunto discreto de pontos. Outro exemplo, que ocorre diversas vezes neste trabalho, é a soma de um número finito de termos de uma expansão em série que em princípio seria infinita.

A validação dos resultados numéricos consiste na comparação entre dados observados e valores calculados, verificando se o erro obtido está dentro de uma faixa aceitável, dadas as características dos dados físicos e a precisão do modelo. Outra forma de validação de um modelo numérico é a comparação do mesmo com uma solução exata das equações governantes, dadas as mesmas condições de contorno, as quais o modelo numérico deve se aproximar. Quanto mais próxima for da solução exata ou dos dados físicos o modelo numérico, melhor o modelo.

Dois indicadores de erro foram usados: o erro absoluto e o erro relativo. O erro absoluto é definido como o módulo da diferença entre os valores da solução exata e da solução numérica, ou entre dois valores de soluções numéricas, para pontos previamente definidos:

$$
e_{a b s}=\sum_{i} a b s\left(\operatorname{Sol}_{\text {exata }}\left(x_{i}\right)-\operatorname{Sol}_{\text {num }}\left(x_{i}\right)\right)
$$

para o qual o erro absoluto máximo é dado por

$$
e_{a b s / \max }=\operatorname{MAX}\left(e_{a b s}\right)
$$

O erro relativo é dado pela soma do módulo da diferença entre os valores da solução exata e numérica ou numéricas, dividido pelo valor da solução exata, para pontos definidos:

$$
e_{\text {rel }}=\sum_{i} \frac{a b s\left(\operatorname{Sol}_{\text {exata }}\left(x_{i}\right)-\operatorname{Sol}_{\text {num }}\left(x_{i}\right)\right)}{\operatorname{Sol}_{\text {exata }}\left(x_{i}\right)}
$$


e o erro relativo máximo é dado por

$$
e_{\text {rel/max }}=\operatorname{MAX}\left(e_{\text {rel }}\right)
$$

Mapas de valores de erro são obtidos, avaliando o erro absoluto e o relativo para os pontos de controle do domínio. Para cada ponto do domínio, o erro absoluto ou relativo é calculado, e o resultado plotado em gráfico. Os erros médios absolutos e relativos são normalizados pela área de cada mapa de erro.

\subsubsection{Convergência Numérica}

Em processos iterativos de solução, os coeficientes desconhecidos do sistema são recalculados a cada iteração, usando os valores obtidos na iteração anterior. A convergência é atingida quando a mudança dos coeficientes entre uma iteração e a anterior é menor que um valor pré-determinado. Para as inomogeneidades circulares e as line doublets, a diferença entre as iterações dos coeficientes da expansão em série de cada elemento é calculada. O processo iterativo só é interrompido quando todos os coeficientes de todos os elementos convergem. Os coeficientes de um elemento são comparados entre as iterações usando o erro absoluto e relativo, dados pelas equações (6.116) e (6.118).

A seção (6.6.7) mostra que, para determinadas partições da matriz, a convergência é garantida para qualquer vetor inicial, desde que o raio espectral da partição seja menor que um. Entretanto, sistemas apresentaram aparente convergência para a solução mesmo se o raio espectral for maior que um. Qualquer sistema que apresente raio espectral maior que um não pode convergir. Um dos possíveis motivos para isto é dado pelo algoritmo de parada: o sistema não convergiu, mas como a diferença entre duas iterações, usado como um dos critérios do algoritmo de parada, não nota mudança, a execução é parada. 


\section{$7 \quad$ Resultados}

Neste capítulo são apresentados os resultados das simulações obtidos com os elementos apresentados no capítulo (5) e os algoritmos de solução apresentados no capítulo (6). O sistema a ser simulado consiste em um domínio horizontal com coordenadas $(x, y)$, com um campo de escoamento uniforme dado por (5.1) de intensidade $Q_{0}$ e ângulo $\beta_{u f}$ com relação ao eixo $x$. O aquífero é composto por um meio poroso de condutividade hidráulica $K$, e o mesmo é considerado livre. A figura (7.1) mostra um esquema do escoamento uniforme em relação ao eixo $x$.

\section{Dados de Entrada para a Simulação}

As tabelas do lado de cada gráfico apresentam os principais valores dos parâmetros usados em cada simulação. Para as inomogeneidades circulares, $K^{+}$representa a condutividade hidráulica, $R$ o raio, $n$ a ordem da expansão em série e $\beta_{u f}$ a direção do campo de escoamento uniforme. Para as inomogeneidades poligonais, novamente $K^{+}$é a condutividade hidráulica da inomogeneidade, $n$ a ordem da expansão em série do potencial, $j$ a ordem da expansão em série do termo de campo distante e $\beta_{u f}$, novamente, a direção do campo distante com relação ao eixo $x$. Com as fraturas, a tabela contém os termos $K^{+}$, $n$, e $\beta_{u f}$, iguais aos elementos anteriores, e $b$, a abertura da fratura. Os valores de distância são dados em metro. Todas as simulações apresentadas neste capítulo usam os dados a seguir:

- Condutividade hidráulica de Fundo: $K=1 e-5 \mathrm{~m} / \mathrm{s}$

- Escoamento uniforme: $Q_{0}=1 e-4 m^{2} / \mathrm{s}$

- Aquífero não confinado (livre)

- Ponto de referência: $(1000,1000)$, carga hidráulica de referência: $\phi(1000,1000)=200 m$

O valor de condutividade hidráulica foi retirado de $(50,52)$ para arenito. Este valor foi escolhido considerando que as fraturas serão incluídas diretamente através do elemento analítico para fraturas. 


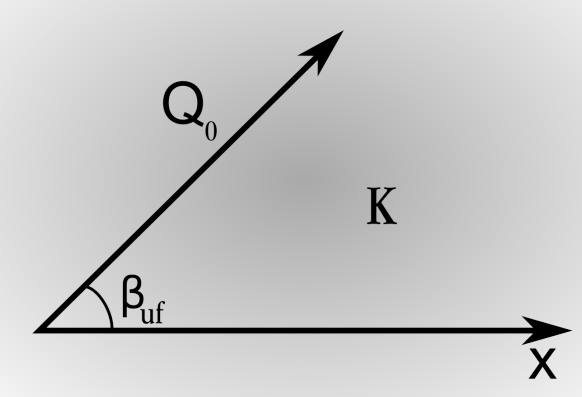

Figura 7.1 - Esquema demonstrando a relação da direção com o escoamento uniforme e o domínio horizontal.

\section{Geração de Gráficos}

Os gráficos usados nesta seção são gerados a partir da própria simulação em Python. Uma vez que a solução for encontrada, utiliza-se a biblioteca Matplotlib (103) para a geração dos gráficos. Para cada ponto do gráfico, a influência de todos os elementos presentes no sistema deve ser calculada e somada. O número de isolinhas é constante para cada tipo de gráfico. Para os gráficos de valores de carga hidráulica e comparação entre isolinhas de carga hidráulica de dois elementos distintos, 40 isolinhas são usadas. Os gráficos de linhas de corrente são gerados com 50 isolinhas. Uma comparação entre o tempo de solução do problema e tempo de visualização do resultado é feita em cada seção.

\section{Comparação entre Soluções para Elementos Analíticos Distintos}

A solução exata para uma inomogeneidade circular em um campo de escoamento uniforme é conhecida, de acordo com a referência (1), e pode ser comparada com o resultado das simulações descritas na seção (7.1). A solução exata da inomogeneidade circular também pode ser comparada com a solução para a inomogeneidade poligonal, construindo um polígono de forma circular. A concordância entre a simulação usando uma inomogeneidade poligonal formada por line doublets e a solução exata para a inomogeneidade circular indica um resultado correto da simulação para inomogeneidades poligonais.

A simulação desenvolvida para o elemento para fratura é comparada com a solução exata para uma fratura, apresentada na referência (4) e descrita na seção (5.4). Uma comparação entre a simulação para a fratura e uma inomogeneidade poligonal formada por line doublets é possível, devido ao comportamento similar de ambas. 


\section{Análise de Erro e Convergência}

Usando as definições da seção (6.9), o erro absoluto aceitável foi definido como $e_{a b s}=1 e-3 m$. Este erro foi definido considerando que o mesmo corresponde a uma diferença de $1 e-3 m$ entre os valores de carga entre duas simulações sendo comparadas. O erro relativo aceitável foi definido como $1 e-5$. Este valor significa que o erro relativo entre os dois resultados sendo comparados é de, por exemplo, $1 e-5 \times 100 \%=0.001 \%$, em metros. Em um gráfico de 100 isolinhas separadas igualmente é possível visualizar erros da ordem 0.05 para erros absolutos e $1 e-3$ para erros relativos.

A convergência é obtida quando o erro absoluto para os coeficientes da expansão em série dos elementos entre duas iterações é menor que o valor pré-determinado, para todos os coeficientes, para todos os elementos:

$$
\sum_{m} \sum_{n}\left(a_{m, n}^{k+1}-a_{m, n}^{k}\right)<e_{s e t}
$$

$a_{m, n}$ é o coeficiente de ordem $n$ do elemento $m$ sendo calculado, e $k$ denota a iteração. $e_{s e t}$ é o valor pré-determinado para que a iteração seja interrompida, e o resultado considerado convergente.

\subsection{Inomogeneidades Circulares}

\subsubsection{Comparação da Solução obtida usando o Algoritmo Iterativo Im- plícito e o Matricial com a Solução Exata}

Esta seção apresenta a comparação da solução exata apresentada na seção (5.2.2) para uma inomogeneidade circular com a solução da simulação para uma inomogeneidade circular obtida através do método de iteração implícito, apresentado na seção (6.6.1). O método de integração escolhido para a equação (6.15a) foi a quadratura gaussiana, fornecido pelo pacote Scipy (98). A figura (7.3) mostra isolinhas de carga, com o valor de cada isolinha no gráfico, e uma escala de cores para a variação de carga. Os valores dos parâmetros usados nesta simulação estão na tabela (7.1).

Tabela 7.1 - Parâmetros para a simulação da figura (7.2).

\begin{tabular}{|l|c|c|c|c|c|}
\hline & $K^{+}(m / s)$ & $R(m)$ & $n$ & Erro Abs. Máx. & Erro Rel. Máx. \\
\hline Iterativo Implícito & 0,001 & 20 & 2 & $1,13 e-13$ & $4,68 e-16$ \\
\hline Matricial & 0,001 & 20 & 2 & $1,13 e-13$ & $4,68 e-16$ \\
\hline
\end{tabular}




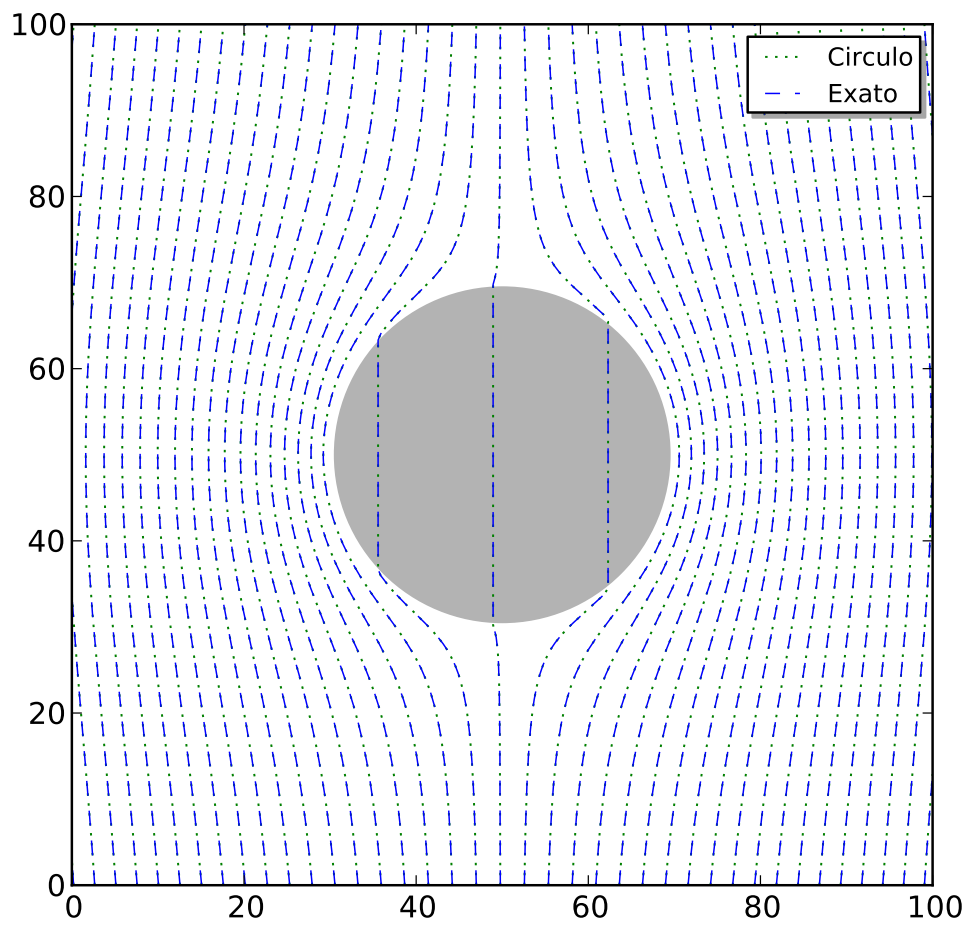

Figura 7.2 - Comparação dos valores de carga hidráulica entre a solução exata e a obtida através do método iterativo implícito.

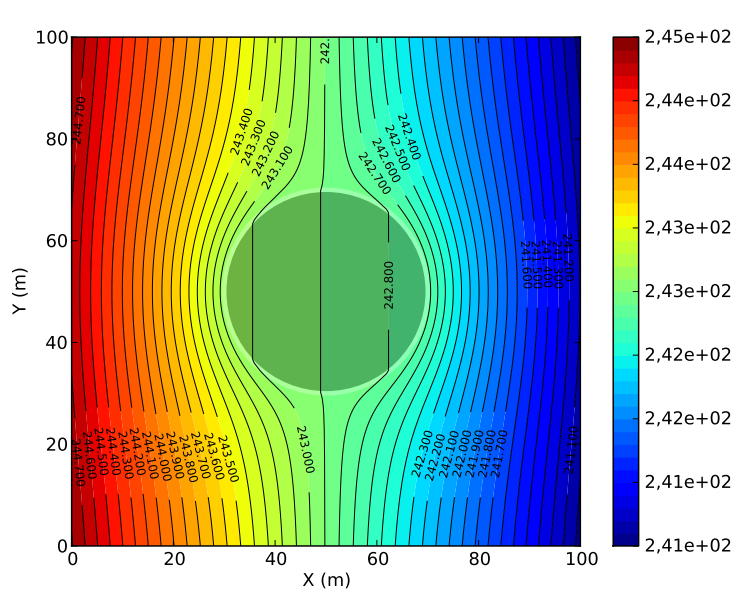

(a) Valores de carga hidráulica

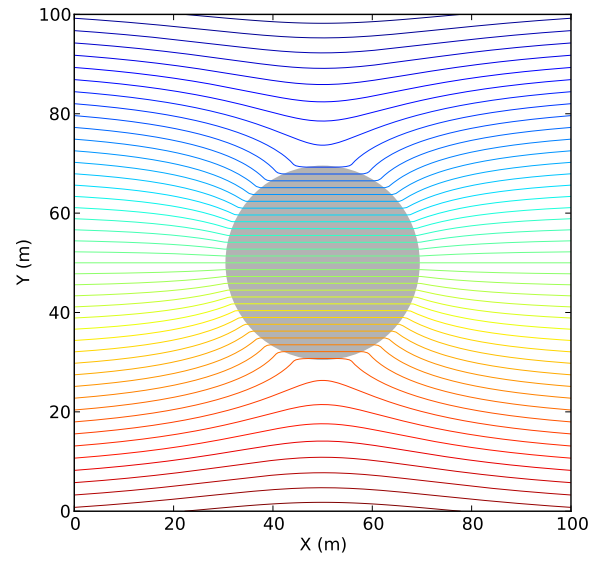

(b) Linhas de Corrente

Figura 7.3 - Valores de carga e linhas de corrente para a simulação da inomogeneidade circular calculado pelo método iterativo implícito.

A figura (7.2) mostra a comparação das isolinhas de carga entre a solução iterativa implícita usando integração numérica para obter os coeficientes e a solução exata. Observa-se que as isolinhas se sobrepõem. A concordância entre a solução iterativa e a solução exata é excelente, com erro absoluto máximo igual a 4,68e-13 e erro relativo máximo de 4,68e-16. A comparação entre o método matricial e a solução exata também teve erro absoluto igual a 4,68e-14 e erro relativo de $(4,68 e-16)$. Este resultado indica que o método iterativo implícito, assim como a solução matricial, usando integração numérica para obtenção dos coeficientes, é capaz de obter um resultado quase 
idêntico à solução exata. Não houve diferença nos resultados ao se aplicar a abordagem de mínimos quadrados ou de integração numérica, tanto na forma matricial quanto na forma iterativa, sendo equivalentes. O tempo computacional de execução para solução do problema e geração dos gráficos é inferior a vinte segundos.

\subsubsection{Simulação para várias Inomogeneidades Circulares}

Várias inomogeneidades circulares podem ser simuladas no mesmo domínio, com diferentes raios e condutividades hidráulicas, maiores ou menores que a do meio poroso. Esta seção apresenta a simulação de 4 inomogeneidades circulares com raios variando de 5 a 25 metros e condutividade hidráulica variando também, de acordo com a tabela (7.2) .

Tabela 7.2 - Parâmetros para a simulação das figuras (7.2) e (7.4).

\begin{tabular}{|l|c|c|c|}
\hline & $K^{+}(\mathrm{m} / \mathrm{s})$ & $R(m)$ & $n$ \\
\hline Ino. Circular 1 & 0,000008 & 15 & 5 \\
\hline Ino. Circular 2 & 0,001 & 25 & 5 \\
\hline Ino. Circular 3 & 0,0002 & 5 & 5 \\
\hline Ino. Circular 4 & 0,0005 & 10 & 5 \\
\hline
\end{tabular}

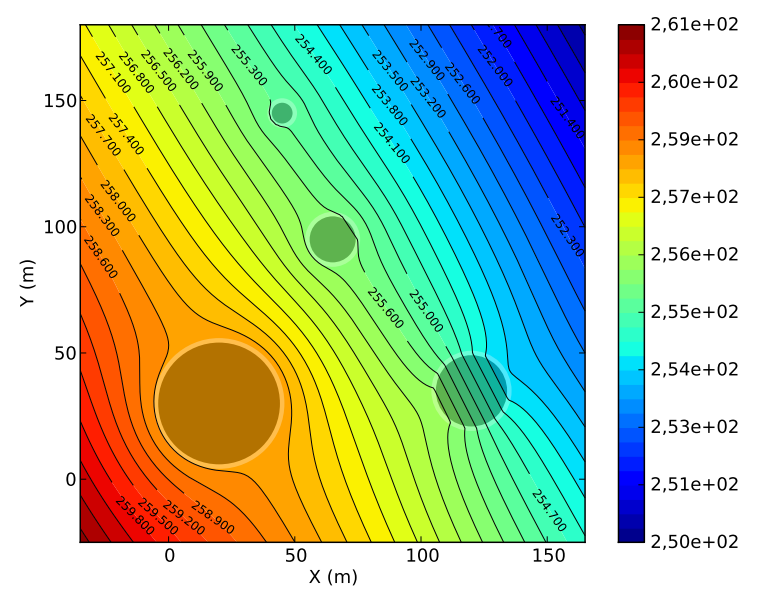

(a) Valores de carga

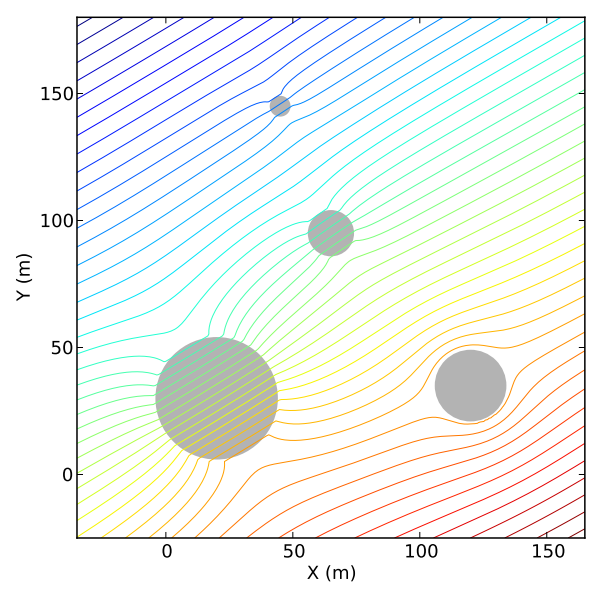

(b) Linhas de corrente

Figura 7.4 - Inomogeneidades circulares com diferentes condutividades hidráulicas e raios, com o escoamento uniforme na direção $\frac{\pi}{6}$ radianos. Valores de carga hidráulica e linhas de corrente para o sistema com parâmetros na tabela (7.2).

A figura (7.4) mostra as linhas de corrente e os valores de carga hidráulica para o sistema de quatro inomogeneidades. Nota-se o comportamento das linhas de corrente com a variação da condutividade hidráulica: as inomogeneidades com condutividade hidráulica menor que a do meio apresenta uma diminuição o número de linhas de corrente em seu interior, enquanto 
inomogeneidades com condutividade hidráulica maior que a do meio apresentam um número maior de linhas de corrente convergindo para o seu interior. Este comportamento é explicado pela maior resistência ao escoamento que a inomogeneidade com condutividade hidráulica menor que a do meio encontra, e pela menor resistência ao escoamento que as inomogeneidades de condutividade hidráulica maior que o meio apresentam. O sistema iterativo implícito funciona para o caso de variação de condutividade hidráulica, tanto maior quanto menor que a do meio, para raios distintos, e com o campo de escoamento uniforme com ângulo $\beta_{u f}$ em relação ao eixo $x$, como visto na figura (7.4), demonstrando sua flexibilidade. O método iterativo implícito converge para este problema em 6 iterações. O tempo de resolução do sistema é de 3,5 segundos, e o tempo de geração dos gráficos de 72 segundos.

\section{Método Iterativo Explícito}

O uso do método iterativo explícito permite a análise do raio espectral para o sistema matricial. O raio espectral de todos os sistemas matriciais montados foi menor que 1 , indicando que a convergência é garantida e deve ser relativamente rápida. Todos os sistemas testados com inomogeneidades circulares convergiram em menos de 12 iterações, o que está de acordo com (93). O uso da abordagem de mínimos quadrados da seção (6.3.2) não forneceu resultados melhores e não houve diminuição do tempo computacional em relação ao uso de integração numérica.

\subsection{Line Doublets para Inomogeneidades Poligonais}

Os resultados para as line doublets são apresentados a seguir. Foi feita uma comparação entre uma inomogeneidade circular poligonal, que consiste em 20 line doublets formando um icoságono, com a solução exata para uma inomogeneidade circular. A concordância entre os dois sistemas é satisfatória, e portanto permite a aplicação de line doublets como inomogeneidades poligonais para a subsequente comparação com fraturas. As simulações (7.2.1) e (7.2.2) foram resolvidas usando tanto o método matricial (6.6.2) quanto o método iterativo implícito (6.6.1) e a solução de ambos foi comparada. O método iterativo explícito (6.6.7) também foi usado no estudo da convergência e número de iterações. Um estudo do número de coeficientes da expansão em série necessários para uma melhor reprodução da continuidade da carga hidráulica também foi feito na seção (7.2.3).

\subsubsection{Comparação da Solução Exata para uma Inomogeneidade Circu- lar com a Aproximação Poligonal por line doublets}

A solução de line doublets que formam um círculo são comparadas com a solução exata de uma inomogeneidade circular (5.2.2). O icoságono é formado por 20 line doublets. Este valor foi escolhido baseado no estudo feito por (55) para o erro na discretização de um círculo em função do número de line doublets. O erro proveniente da discretização do círculo poligonal é ressaltado nos pontos de junção entre as line doublets, sendo esta a maior fonte de erro na comparação. 
Tabela 7.3 - Parâmetros para a simulação da comparação entre a solução exata para uma inomogeneidade circular e a aproximação por um icoságono usando line doublets.

\begin{tabular}{|l|c|c|c|c|}
\hline & $K^{+}(m / s)$ & $R(m)$ & $n$ & $j$ \\
\hline Iterativo Implícito & 0,0001 & 20 & 5 & 250 \\
\hline Matricial & 0,0001 & 20 & 5 & 250 \\
\hline
\end{tabular}

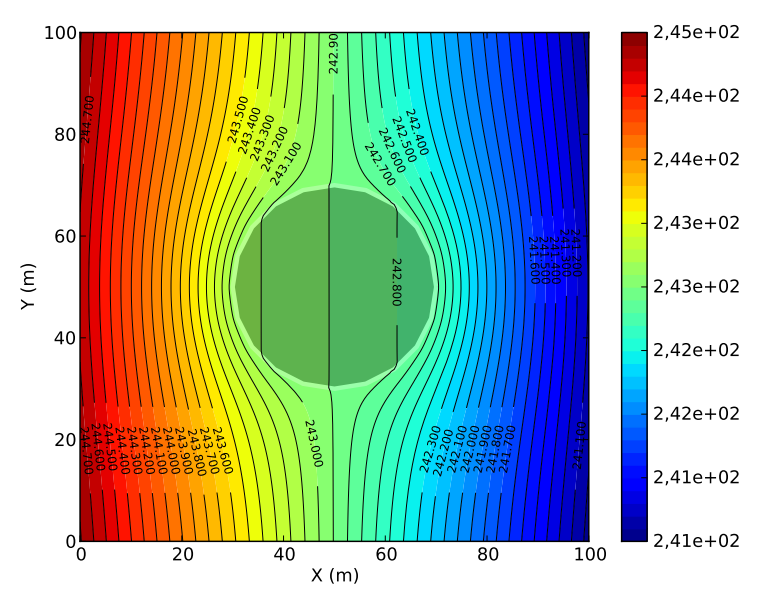

(a) Valores de carga

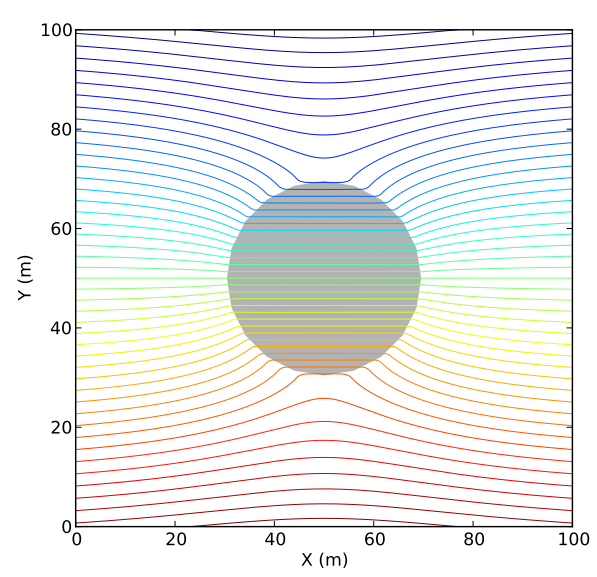

(b) Linhas de corrente

Figura 7.5 - Inomogeneidade circular formada por 20 line doublets. Valores de carga hidráulica e linhas de corrente para o sistema com parâmetros descritos na tabela (7.3).

A figura (7.5) apresenta os valores de carga hidráulica e linhas de corrente para a simulação de uma inomogeneidade poligonal na forma de icoságono, formada por 20 line doublets. As isolinhas de carga hidráulica apresentam comportamento semelhante aos da solução para uma inomogeneidade circular, apresentada na figura (7.3). A figura (7.6) apresenta a comparação das isolinhas de carga entre a solução da inomogeneidade poligonal em forma de icoságono e a solução exata para a inomogeneidade circular para o método matricial. A concordância de ambas apresenta pequeno erro, o que pode ser verificado na tabela (7.4). Os mapas de erro (7.7) para o método matricial indicam que a principal fonte de erro é a discretização da inomogeneidade circular em 20 line doublets. Os maiores erros se encontram na junção dos elementos. Este comportamento também foi verificado e é muito similar ao se usar o método iterativo implícito. 


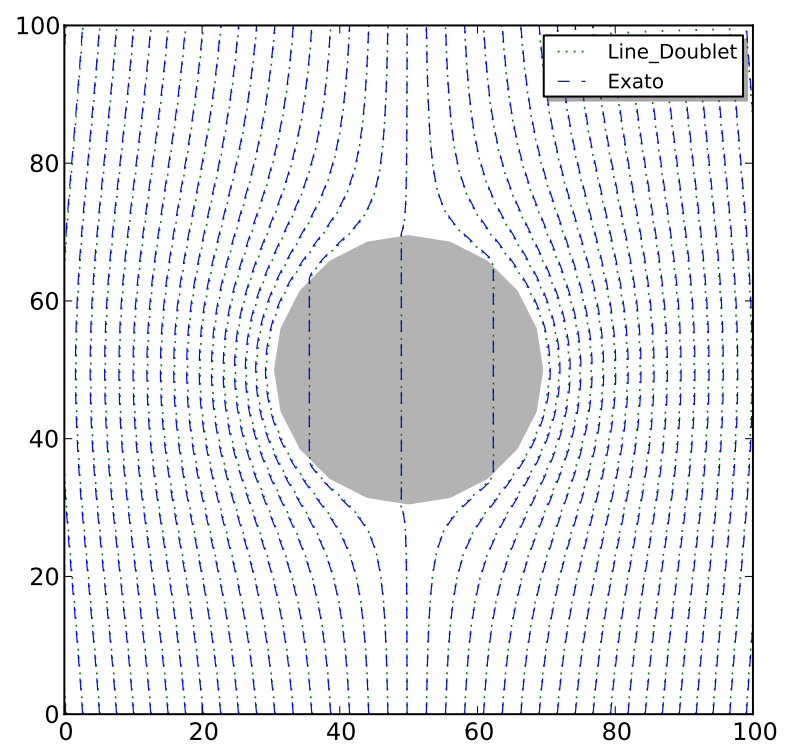

Figura 7.6 - Inomogeneidade circular poligonal composta por 20 line doublets (icoságono). Comparação de isolinhas de carga entre a solução aproximada da inomogeneidade circular poligonal e a solução exata para uma inomogeneidade circular. A concordância entre as duas soluções é aceitável, apesar de se notar erros próximo à borda do círculo.

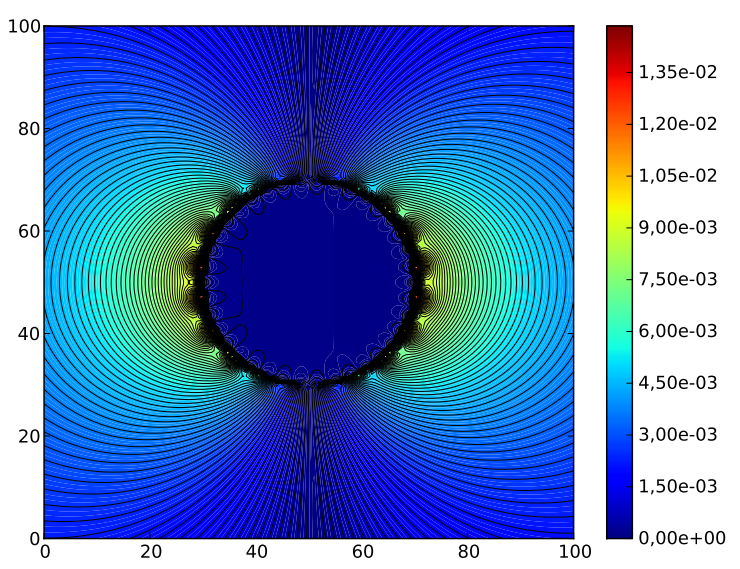

(a) Mapa de erro absoluto

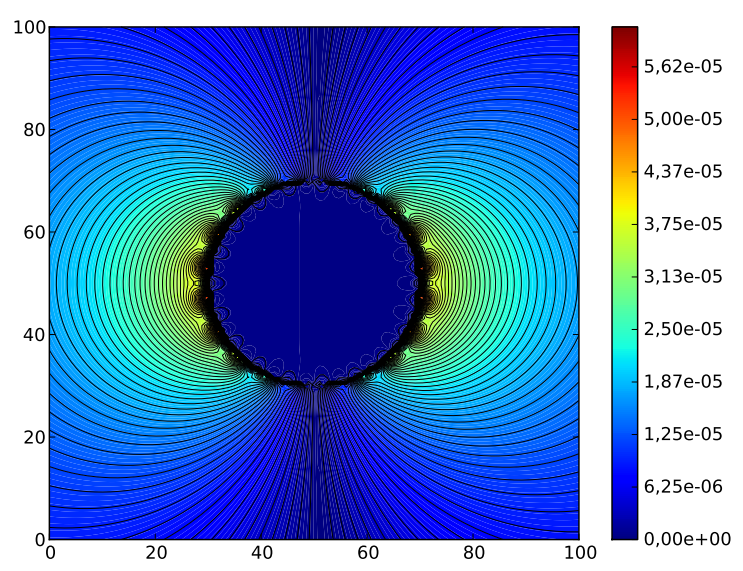

(b) Mapa de erro relativo

Figura 7.7 - Inomogeneidade circular poligonal discretizada por 20 line doublets. Mapa de erro absoluto e relativo entre a solução exata e a solução aproximada pelo icoságono formado por line doublets. Os maiores erros ocorrem nos pontos de junção entre as line doublets.

Tabela 7.4 - Erros absolutos máximos, absolutos médios, relativos máximos e relativos médios para uma inomogeneidade circular e a aproximação por um icoságono usando line doublets. Comparação entre a solução obtida pelos métodos iterativo implícito e o matricial com a solução exata. O erro para os valores no interior à inomogeneidade é próximo de zero.

\begin{tabular}{|l|c|c|c|c|}
\hline & Err. Abs. Med. & Err. Abs. Máx & Err. Rel. Med & Err. Rel. Máx \\
\hline Iterativo Implícito & $3,61 \mathrm{e}-3$ & $1,48 \mathrm{e}-2$ & $1,30 \mathrm{e}-5$ & $6,09 \mathrm{e}-5$ \\
\hline Matricial & $3,16 \mathrm{e}-3$ & $1,48 \mathrm{e}-2$ & $1,30 \mathrm{e}-5$ & $6,09 \mathrm{e}-5$ \\
\hline
\end{tabular}


Os valores da comparação entre os resultados do método iterativo implícito para a inomogeneidade na forma de icoságono com a solução exata para uma inomogeneidade circular e o matricial com a solução exata para a forma de icoságono, apresentados na tabela (7.4), demonstram que ambos os métodos apresentam uma boa comparação com a solução exata, indicando a equivalência de ambos os métodos de solução no que tange a aproximação para uma inomogeneidade circular.

\section{Comparação entre o Método Iterativo Implícito e o Matricial para a Solução de uma Inomogeneidade Circular formada por line doublets}

O tempo de processamento foi comparado para o método iterativo implícito e o método matricial, na solução da inomogeneidade poligonal em fomra de um icoságono, composta por 20 line doublets. O tempo de processamento para a solução matricial é dividido na construção da matriz, na solução do sistema, e o restante do tempo é gasto na plotagem dos gráficos. Para o método iterativo implícito, o tempo é dividido no processo iterativo, onde cada iteração demora um tempo $t_{i}$, e a solução é obtida em $m$ iterações. O tempo total de computação é dado por $t_{i} m$. O procedimento de plotagem dos gráficos é o mesmo para a solução matricial. A tabela (7.5) apresenta os valores de tempo de montagem da matriz, processamento, iteração e construção dos gráficos para o método iterativo implícito e o método matricial, em segundos, para o icoságono descrito na seção (7.2.1). Os parâmetros para esta comparação são dados pela tabela (7.3).

Tabela 7.5 - Comparação entre os tempos de processamento para a construção da matriz e a solução do sistema linear resultante no método matricial e o tempo de iteração para o método iterativo implícito. O tempo de construção dos gráficos também é apresentado.

\begin{tabular}{|c|c|c|c|c|c|}
\hline & Const. Mat. (s) & Sol. Sist.(s) & Iter. (s) & Const. Gráf. (s) & Total (s) \\
\hline Matricial & 81,29 & 0,30 & - & 663,09 & 744,68 \\
\hline Iterativo Implícito & - & - & 3903,46 & 690,47 & 4539,71 \\
\hline
\end{tabular}

O tempo para solução do problema usando o método iterativo implícito, como observado na tabela (7.5), é muito superior ao tempo necessário para a solução ser encontrada usando o método matricial. O método matricial tem gasto de tempo somente na construção da matriz, já que a solução do sistema linear resultante para todos os casos estudados foi inferior a $0,1 \mathrm{~s}$, para matrizes de dimensões da ordem de $N * n_{\text {elem }}+1$, onde $N$ é o número de elementos analíticos presentes, $n_{\text {elem }}$ a ordem da expansão do elemento, e o termo adicional sendo necessário para se calcular a constante livre. Os gráficos são construídos calculando a influência de todos os elementos para cada ponto do domínio, de acordo com o princípio de superposição apresentado na seção (4.2.1). Os tempos de construção do gráfico são muito próximos, já que o procedimento de construção do gráfico é independente do método de solução escolhido. Os resultados obtidos na comparação entre os algoritmos de solução matricial e iterativo implícito são apresentados na tabela (7.6). 
Tabela 7.6 - Valores de erro absoluto e erro relativo para a comparação entre o método iterativo implícito e o método matricial.

\begin{tabular}{|c|c|c|c|c|}
\hline & Err. Abs. Med. & Err. Abs. Máx. & Err. Rel. Med. & Err. Rel. Máx. \\
\hline Comparação & 6,09e-09 & 6,42e-8 & $2,71 \mathrm{e}-11$ & 2,50e-11 \\
\hline
\end{tabular}

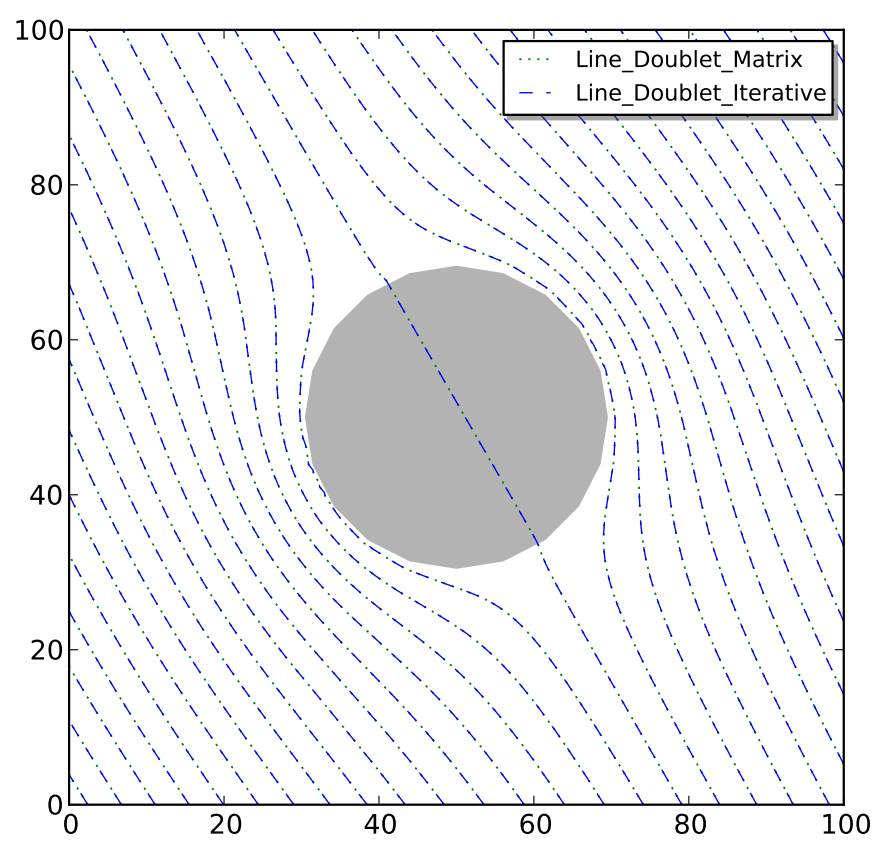

Figura 7.8 - Inomogeneidade circular poligonal composta por 20 line doublets. Comparação das soluções geradas pelo algoritmo matricial e iterativo implícito para a inomogeneidade poligonal, com escoamento uniforme na direção $\pi / 3$ radianos. A concordância entre ambas é excelente.

O erro absoluto máximo entre ambos é de $6,48 e-8$, e o erro relativo máximo é igual a 2,50e-11, mostrando uma excelente concordância entre o método iterativo e o método matricial. A figura (7.8) mostra a comparação das soluções obtidas pelo método iterativo implícito e o matricial, os quais simulam formam uma inomogeneidade circular poligonal.

Considerando que o erro entre as dois algoritmos de simulação e a solução exata são muito semelhantes e que o tempo de processamento do método matricial é muito inferior ao tempo de processamento do método iterativo implícito, o método matricial se apresenta como uma melhor escolha. O custo de memória do método matricial é superior na proporção do dobro do custo de processamento via algoritmo iterativo implícito. Entrentanto, com o overhead que o próprio Python necessita para armazenar as variáveis de ambiente, tal aumento de custo, para a dimensão do sistema simulado, não é significativo. Como visto acima, as dimensões da matriz são da ordem de $N * n_{\text {elem }}+1$ : um sistema com 20 elementos line doublet, cada elemento de ordem 5 , geraria uma matriz de dimensões $26 \times 26$, com 676 elementos de matriz. 


\subsubsection{Simulação para Duas Inomogeneidades Poligonais}

Na presente seção, é simulado o escoamento com a presença de duas inomogeneidades poligonais, com condutividades hidráulicas diferentes. Os dados para esta simulação são apresentados na tabela (7.7). $n$ denota o número de coeficientes para o elemento, e $j$ o número de coeficientes usados na expansão de campo distante.

Tabela 7.7 - Parâmetros para a simulação para duas inomogeneidades poligonais quadradas com condutividades hidráulica formadas por line doublets.

\begin{tabular}{|l|c|c|c|}
\hline & $K^{+}(m / s)$ & $n$ & $j$ \\
\hline Quadrado Esquerda & 0,001 & 6 & 250 \\
Quadrado Direita & 0,000002 & 6 & 250 \\
\hline
\end{tabular}

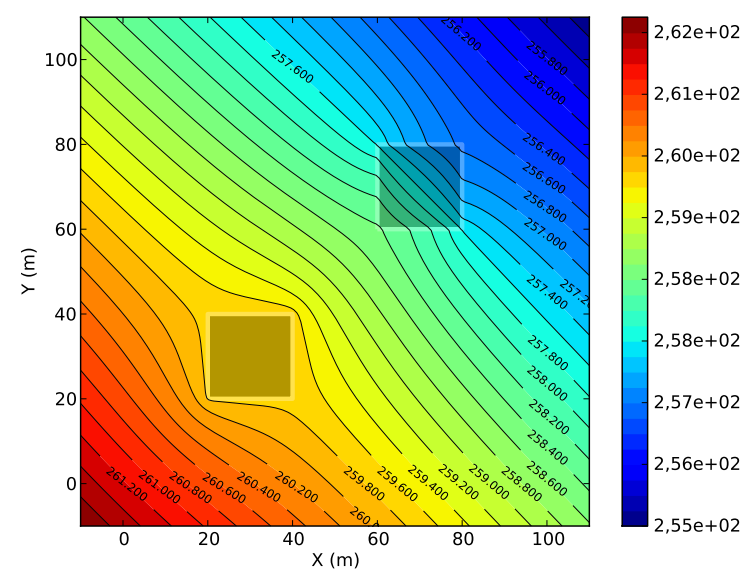

(a) Valores de carga hidráulica

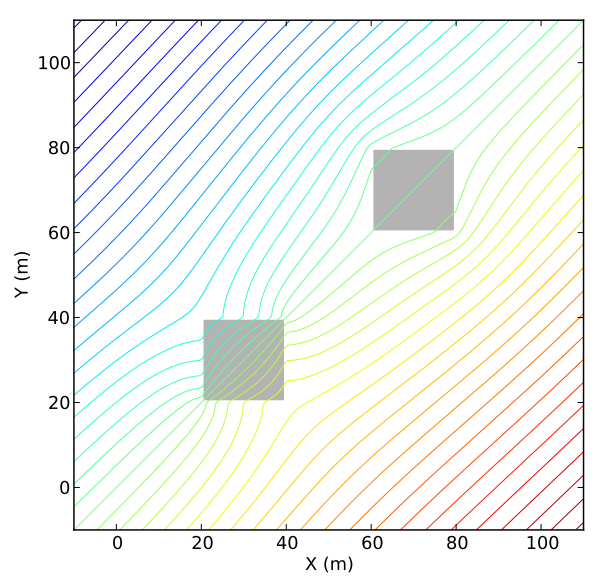

(b) Linhas de corrente

Figura 7.9 - Duas inomogeneidades poligonais quadradas, com condutividades hidráulicas diferentes, em um campo de escoamento uniforme com ângulo de $\pi / 4$ radianos em relação ao eixo $x$. Valores de carga hidráulica e linhas de corrente. A inomogeneidade da direita tem condutividade hidráulica maior que a do meio, e a da esquerda, menor que a do meio.

A figura (7.9) mostra os valores de carga hidráulica e linhas de corrente para as duas inomogeneidades quadradas de condutividade hidráulica diferentes. A diferença nos valores de condutividade hidráulica leva a um diferente comportamento das isolinhas de carga, com uma concentração no interior do elemento para o quadrado com condutividade hidráulica menor e um afastamento para o quadrado com maior condutividade hidráulica. Este comportamento é esperado, indicando a maior velocidade de escoamento para uma inomogeneidade com condutividade hidráulica maior e menor escoamento para uma com condutividade hidráulica menor. 


\subsubsection{Variação na Ordem da Expansão para a line doublet}

A diminuição ou aumento da ordem da expansão $n$ também influencia o resultado. A figura (7.10) mostra um corte através do eixo central dos elementos. Nota-se que o eixo $x$ foi parametrizado entre 0 e 1 , mas representa a linha que corta ambos os elementos da figura (7.15). O aumento da ordem $n$ do elemento leva uma menor descontinuidade na carga na fronteira do mesmo, o que é esperado de acordo com a equação (5.3.1), que apresenta a condição de contorno para uma inomogeneidade na condutividade hidráulica.

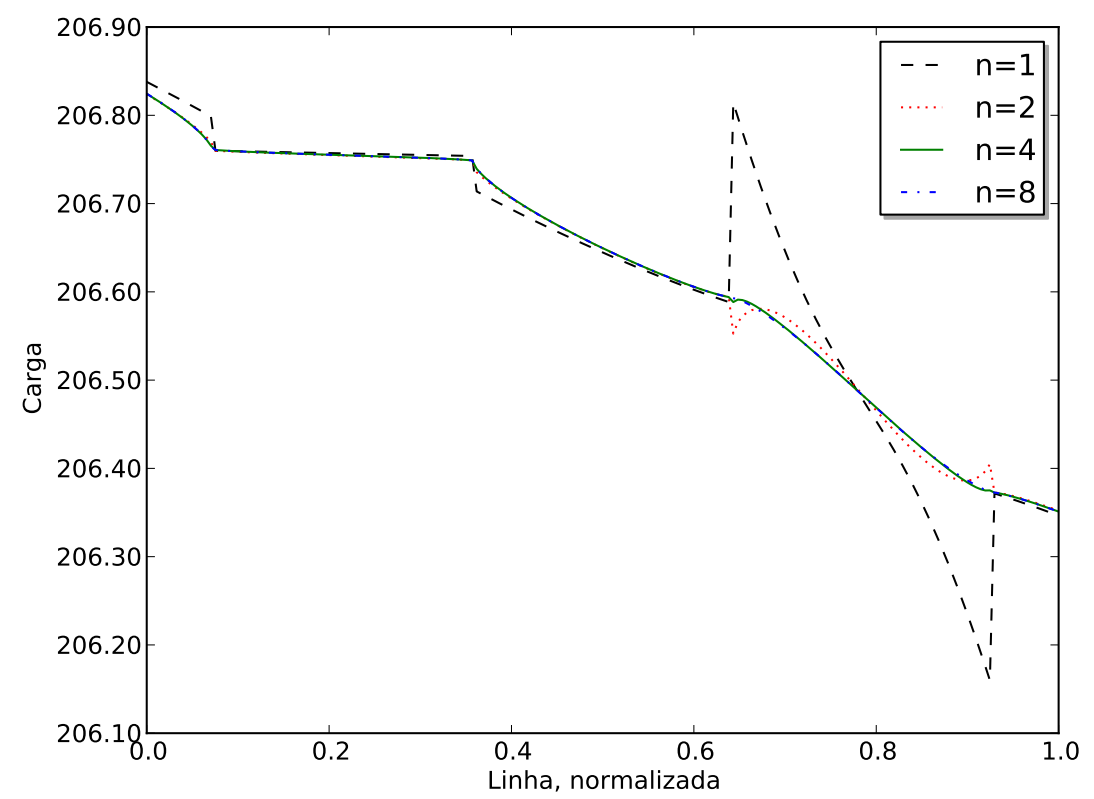

Figura 7.10 - Duas inomogeneidades poligonais em um campo de escoamento uniforme com ângulo de $\pi / 4$ radianos. Valores de carga para uma seção que passa através das duas inomogeneidades. Nota-se que o aumento da ordem do elemento diminui a descontinuidade do potencial, trazendo melhoria ao resultado.

Verifica-se que o aumento do número de coeficientes diminui a descontinuidade da carga na borda do elemento, levando a uma melhor reprodução das condições de contorno. O erro também é proporcional à condutividade hidráulica, com um erro muito maior para a inomogeneidade com cond. hidráulica igual a 30, em relação à de condutividade hidráulica igual a 0,1. O aumento de 4 para 8 coeficientes não traz uma melhoria considerável no resultado. Um valor para $n=5$, portanto, é suficiente para as aplicações aqui apresentadas.

\subsubsection{Algoritmo Iterativo Explícito para a Inomogeneidade Poligonal formada por line doublets}

O algoritmo iterativo explícito da seção (6.6.7) foi utilizado para resolver todos os sistemas lineares resultantes de inomogeneidades poligonais das seções (7.2.1) e (7.2.2). Para todos os sistemas estudados a partição de Gauss-Seidel (6.111) foram menores que 1. Para alguns sistemas a análise do raio espectral demonstrou que dependendo da complexidade da geometria da inomogeneidade e da 
diferença de cond. hidráulica com relação ao meio, o raio espectral para a partição de Jacobi (6.109) foi maior que 1. Mesmo para esses sistemas, a solução convergiu, mas muito lentamente, com alguns sistemas levando até 900 iterações para a solução. Os valores dos coeficientes desconhecidos $a_{j, n}$ para cada line doublet foram iguais aos encontrados pelo método matricial.

\subsection{Fraturas}

Esta seção apresenta os resultados obtidos para a simulação com elementos analíticos para representar domínios fraturados. Os resultados são comparados com os obtidos para line doublets de mesmo perfil elipsóide das fraturas. Os valores de condutividade hidráulica e abertura são calculados usando a lei cúbica, apresentada na seção (3.5.1), dada por

$$
K^{+}=\frac{\rho g}{\mu} \frac{b^{2}}{12}
$$

onde $\rho$ é a densidade da água, $g$ a aceleração da gravidade local, $\mu$ a viscosidade dinâmica e $b$ a abertura da fratura.

A solução matricial e a iterativa explícita são as utilizadas, já que a iterativa implícita apresentou divergências mesmo para sistemas simples.

\section{Divergência do Algoritmo Iterativo Implícito}

O algoritmo iterativo implícito apresentou divergência mesmo em sistemas com elementos para fratura simples, como duas fraturas paralelas e distantes. Os valores com os quais os coeficientes desconhecidos da expansão do potencial para a fratura eram iniciados não influenciaram o comportamento da divergência. A divergência ocorria de forma rápida, com valores divergindo já na terceira iteração. Uma das causas possíveis para a divergência é a soma da descarga de todos os elementos antes da etapa de integração. Na soma, dois valores grandes podem ser somados e o resultado da soma ocasionar overflow, comprometendo a iteração.

O uso de uma aproximação de mínimos quadrados com a matriz quadrada também não levou a melhoras. Estes impedimentos levaram ao desenvolvimento de um sistema matricial de solução e ao estudo de sistemas iterativos explícitos, como o uso do método de Jacobi e Gauss-Seidel. A análise do raio espectral, apresentado na seção (6.6.7), foi utilizada para analisar o algoritmo iterativo explícito para o caso das fraturas.

\subsubsection{Comparação da Solução Analítica para uma Fratura e uma Ino- mogeneidade Poligonal}

A solução analítica para uma fratura (5.4.2) pode ser comparada com a solução matricial para uma inomogeneidade poligonal. A inomogeneidade poligonal modela uma fratura através 
da construção de um perfil elipsóide com as mesmas dimensões de abertura e comprimento da fratura. Entretanto, uma inomogeneidade poligonal formada por line doublets não se apresentou como adequada para simular o comportamento de uma fratura para parâmetros encontrados em campo. A geometria da inomogeneidade apresenta problemas nas extremidades, onde o espaçamento entre duas line doublets de cada lado da elipse é pequeno, causando uma grande influência entre os dois elementos e impossibilitando uma solução correta. Tal comportamento pode ser verificado nas figuras (7.11) e (7.12). Na figura (7.11), a solução analítica para uma fratura é comparada com uma elipse formada por line doublets, mas com parâmetros relativos, dados na tabela (7.8). A inomogeneidade utiliza os mesmos parâmetros que o elemento para fratura.

Tabela 7.8 - Parâmetros relativos para a simulação de uma inomogeneidade elipsóide formada por line doublets e a comparação com a solução analítica para o elemento fratura. Os parâmetros são relativos: $K^{+} / K$ é a relação entre a condutividade hidráulica da fratura e a externa; $L / b$ é a relação entre o comprimento da fratura $L$ e sua abertura $b$.

\begin{tabular}{|c|c|c|c|}
\hline & $K^{+} / K$ & $L / b$ & $n$ \\
\hline Parâmetros & 100 & 100 & 5 \\
\hline
\end{tabular}

Os parâmetros são apresentados de forma relativa: $K^{+} / K$ denota a relação entre a condutividade hidráulica da fratura e a condutividade hidráulica externa; $L / b$ é a relação entre o comprimento da fratura $L$ e sua abertura $b$, e $n$ a ordem do elemento.

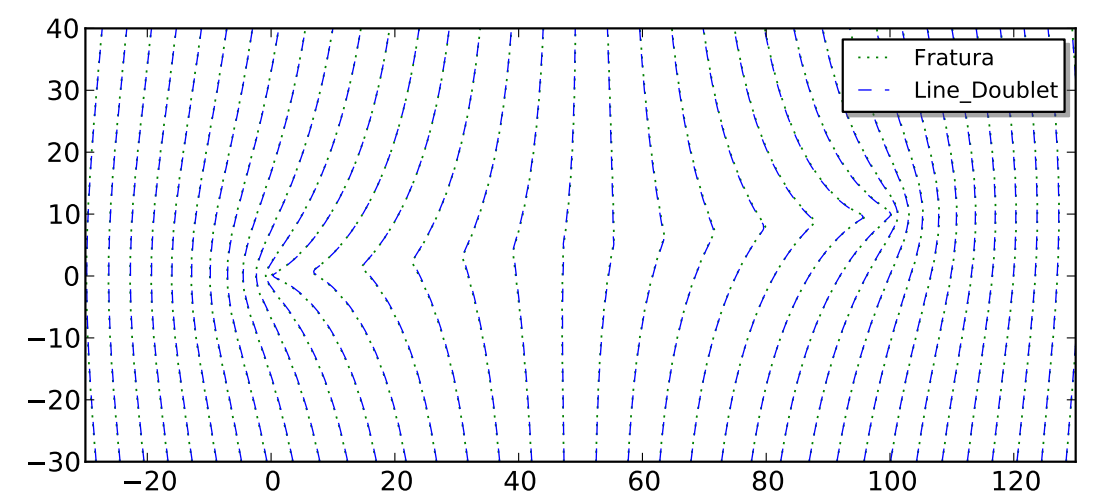

Figura 7.11 - Solução analítica para uma fratura com condutividade hidráulica igual a 100 e abertura igual a 1 , com comprimento igual a 100. Comparação entre os valores de carga para a fratura e uma elipse formada por 28 line doublets. A concordância é excelente.

Como a figura (7.11) mostra, a comparação entre a solução analítica para uma fratura e uma elipse formada por 28 line doublets tem boa concordância, com erro absoluto igual a 0,001 e erro relativo igual a $9,26 e-6$, para parâmetros relativos. Mas, ao se usar parâmetros reais, o elemento line doublet não consegue simular o comportamento correto, como pode ser visto na figura (7.12), dados os parâmetros da tabela (7.9):

Nota-se que os parâmetros para esta fratura são consideravelmente elevados, sendo que uma fratura com abertura de $0,025 \mathrm{~m}$ é uma abertura considerada para uma falha. Mesmo neste caso 
Tabela 7.9 - Parâmetros para uma fratura a ser simulada pelo elemento analítico para uma fratura.

\begin{tabular}{|c|c|c|c|}
\hline & $K^{+}(m / s)$ & $b(m)$ & $n$ \\
\hline Fratura & 0,5 & 0,025 & 5 \\
\hline
\end{tabular}

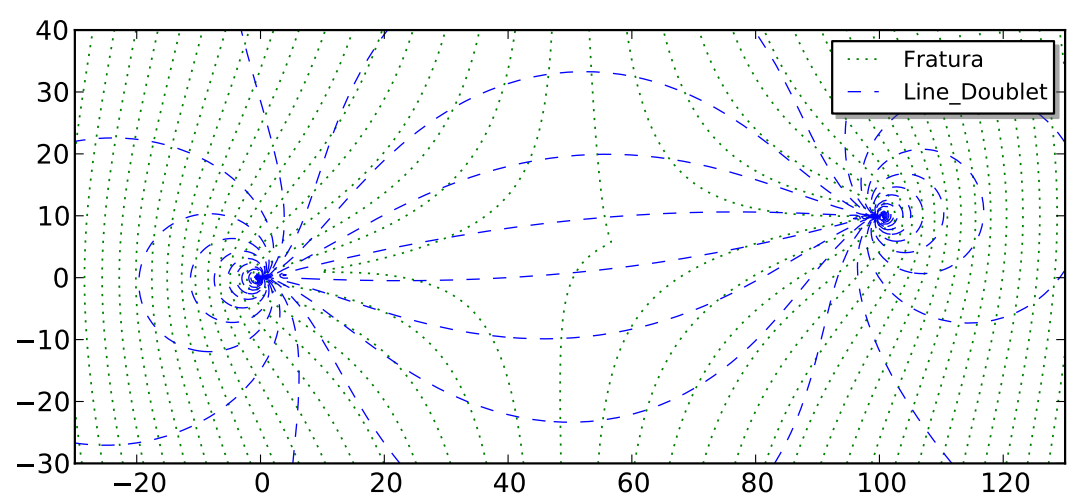

Figura 7.12 - Solução analítica para uma fratura com condutividade hidráulica e abertura dadas pela tabela (7.9). Comparação com uma elipse formada por 28 line doublets e mesmo parâmetros do elemento fratura. O elemento fratura consegue modelar o escoamento, enquanto a line doublet não apresenta um resultado correto.

de limite superior de abertura e condutividade hidráulica da fratura, a simulação via line doublets apresenta um resultado incorreto, como pode ser visto na figura (7.12).

O mesmo procedimento pode ser usado no uso do elemento analítico para mais de uma fratura. Para duas fraturas, a solução numérica para fraturas é aplicada. As figuras (7.13) e (7.14) ilustram este resultado, novamente usando primeiro parâmetros relativos da tabela (7.8), e parâmetros reais, dados na tabela (7.9):

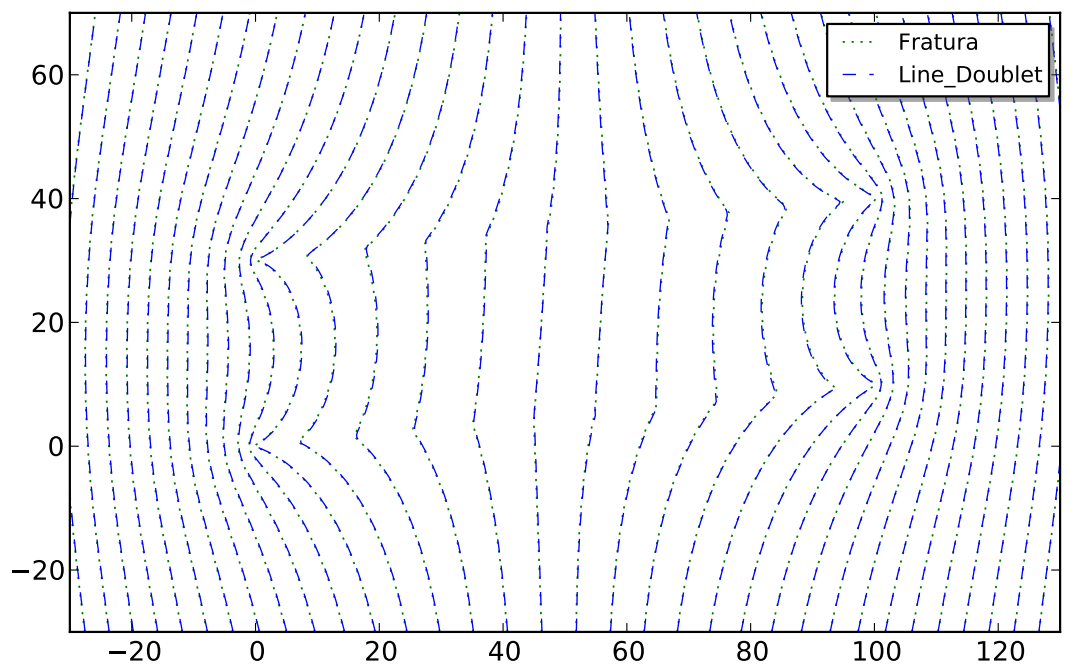

Figura 7.13 - Solução analítica para duas fraturas com condutividade hidráulica igual a 100 e abertura igual a 1, com comprimento igual a 100. Comparação entre os valores de carga para as fraturas e duas elipse formada por 28 line doublets. A concordância também é excelente. 


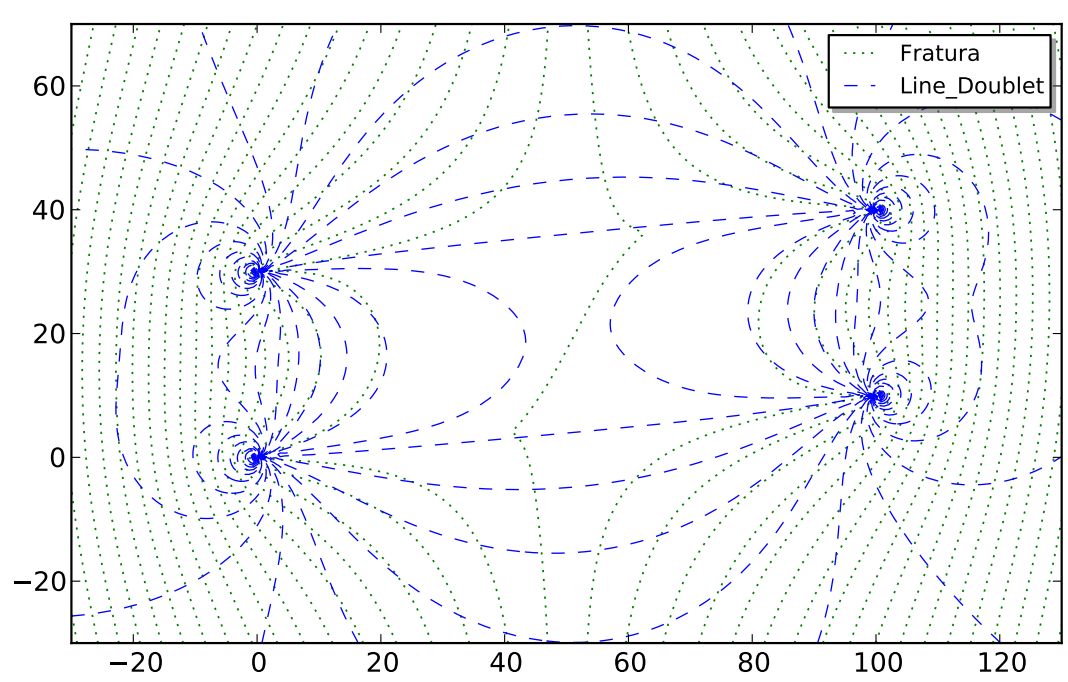

Figura 7.14 - Solução usando o método matricial para duas fraturas com condutividade hidráulica e abertura dadas pela tabela (7.9). Isolinhas de carga hidráulica. Comparação com duas elipses formadas por 28 line doublets e mesmo parâmetros do elemento fratura. O elemento fratura consegue modelar o escoamento, enquanto a line doublet não apresenta um resultado correto.

Pode-se concluir que o elemento fratura apresenta o mesmo comportamento qualitativo que a inomogeneidade elipsóide formada por line doublets. Esta concordância demonstra que o elemento analítico para fraturas representa corretamente o escoamento de acordo com o seu modelo conceitual, de uma fratura preenchida por um material de condutividade hidráulica maior que a do meio. A inomogeneidade elipsóide, entretanto, não consegue simular uma fratura para parâmetros mais próximos dos encontrandos em campo, enquanto o elemento para fratura consegue. O elemento analítico para fraturas, portanto, apresenta um resultado melhor em relação à inomogeneidade quando parâmetros reais são utilizados.

\subsubsection{Algoritmo Iterativo Explícito para o Elemento para Fraturas}

O algoritmo iterativo explícito da seção (6.6.7) também foi aplicado para o elemento analítico para fraturas. Para todos os casos estudados, a partição de Jacobi (6.109) sempre apresentou raio espectral maior que um. Em geral, a partição de Gauss-Seidel apresentou raio espectral menor que um, apesar de ter apresentado alguns casos de raio espectral maior que um, principalmente em casos com cruzamento de fraturas. A convergência para estas simulações, tanto no caso de Jacobi quanto de Gauss-Seidel não foi satisfatória. O processo iterativo para o elemento para fratura apresentou simulações onde a convergência levou até 800 iterações ou não convergiu. O uso de integração numérica ou a abordagem de mínimos quadrados não influenciou a convergência.

\subsubsection{Simulação para cruzamento de Fraturas}

A simulação do escoamento com cruzamento de fraturas foi testada. Entretanto, a condição de contorno no cruzamento, a conservação do vetor de descarga, não foi implementada no potencial 
complexo do elemento. Os gráficos de carga e linhas de corrente parecem indicar o comportamento correto na intersecção de duas fraturas. Um sistema com line doublets com formato retangular foi montado, e o sistema comparado com fraturas. As figuras (7.16) para o cruzamento de duas fraturas de mesma condutividade hidráulica relativas ao meio poroso, dadas pela tabela (7.10):

Tabela 7.10 - Parâmetros relativos para a simulação do cruzamento de dois elementos analíticos para fratura.

\begin{tabular}{|l|c|c|c|}
\hline & $K^{+} / K$ & $L / b$ & $n$ \\
\hline Fratura 1 & 100 & 1 & 5 \\
Fratura 2 & 100 & 1 & 5 \\
\hline
\end{tabular}

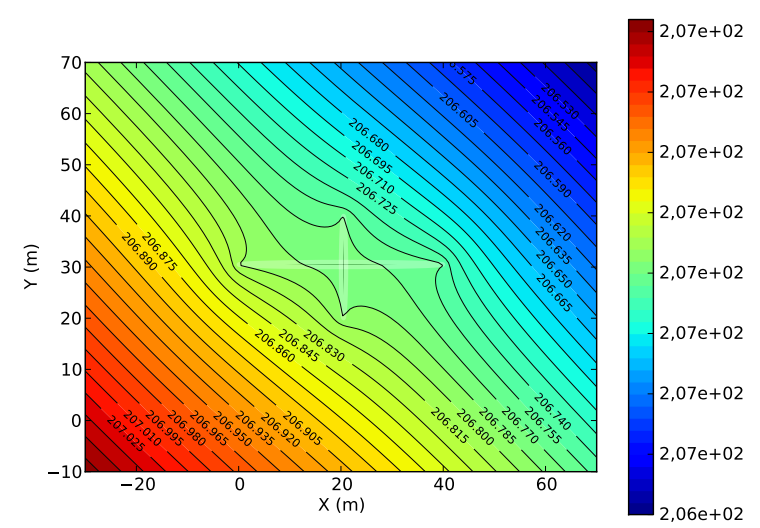

(a) Valores de carga hidráulica

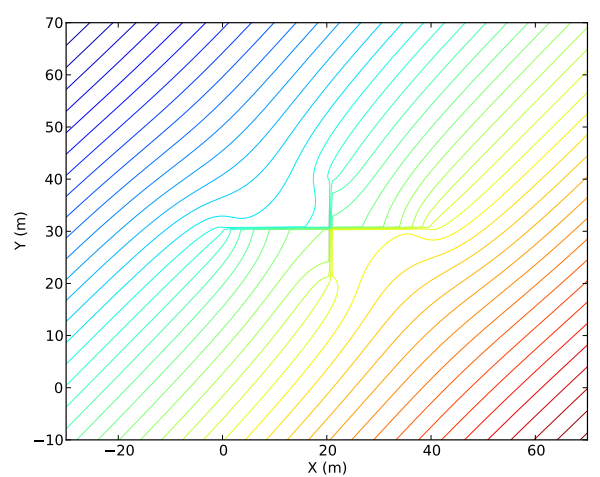

(b) Linhas de corrente

Figura 7.15 - Valores de carga e Linhas de corrente para o cruzamento de dois elementos analíticos para fratura.

A comparação entre as duas simulações apresentou uma diferença absoluta máxima em 0.01, e diferença relativa máxima de $4.50 e-5$. A diferença é maior na comparação das extremidades, onde a fratura tem a extremidade dada pela expressão do potencial (5.82), enquanto este exemplo para a inomogeneidade poligonal a terminação é retangular. Observa-se no gráfico (7.15) que as linhas de corrente na intersecção parecem se comportar corretamente, com parte do escoamento sendo transferido para a outra fratura e parte continuando na mesma fratura originária antes do cruzamento.

Apesar do comportamento no cruzamento ser qualitativamente correto, a conservação do vetor descarga no ponto de intersecção não foi verificada, não permitindo afirmar que a solução é correta. A concordância com o sistema de line doublets indica que a solução aproxima-se da solução esperada, mas a diferença absoluta é mais alto que para os outros casos onde as fraturas e as inomogeneidades poligonais foram comparadas. Os parâmetros utilizados foram novamente relativos, devido à impossibilidade das inomogeneidades poligonais em simular um sistema com parâmetros mais próximos à realidade, como visto na seção (7.3.1). 


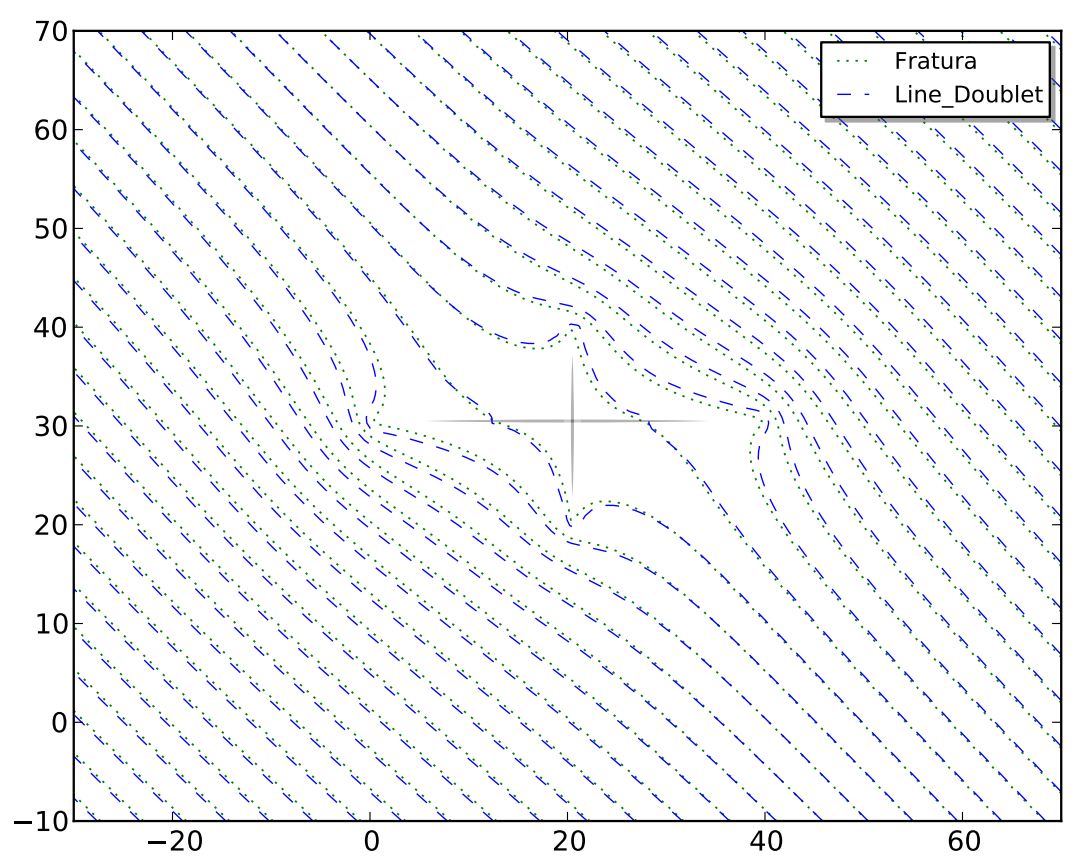

Figura 7.16 - Duas fraturas em cruzamento em um campo de escoamento uniforme a0 radiano. Comparação das isolinhas de carga hidráulica entre um sistema de line doublets e duas fraturaz em cruzamento.

\subsubsection{Simulação de alguns sistemas fraturados}

As figuras ilustram alguns exemplos de sistemas de fraturas, com intersecção. As tabelas apresentam os parâmetros para as simulações, de acordo com a legenda de cada um. Nestes exemplos, a condutividade hidráulica do meio poroso é de $K=1 e-10 \mathrm{~m} / \mathrm{s}$, condizende com a condutividade hidráulica do basalto (52), e o escoamento uniforme tem intensidade $Q_{0}=1 e-9 \mathrm{~m}^{2} / \mathrm{s}$.

Tabela 7.11 - Parâmetros para a simulação de um sistema fraturado, com todas as fraturas de mesma condutividade hidráulica e abertura.

\begin{tabular}{|c|c|c|c|}
\hline & $K^{+}(M / s)$ & $b$ & $n$ \\
\hline Fraturas & $2 e-4$ & 0,0005 & 5 \\
\hline
\end{tabular}




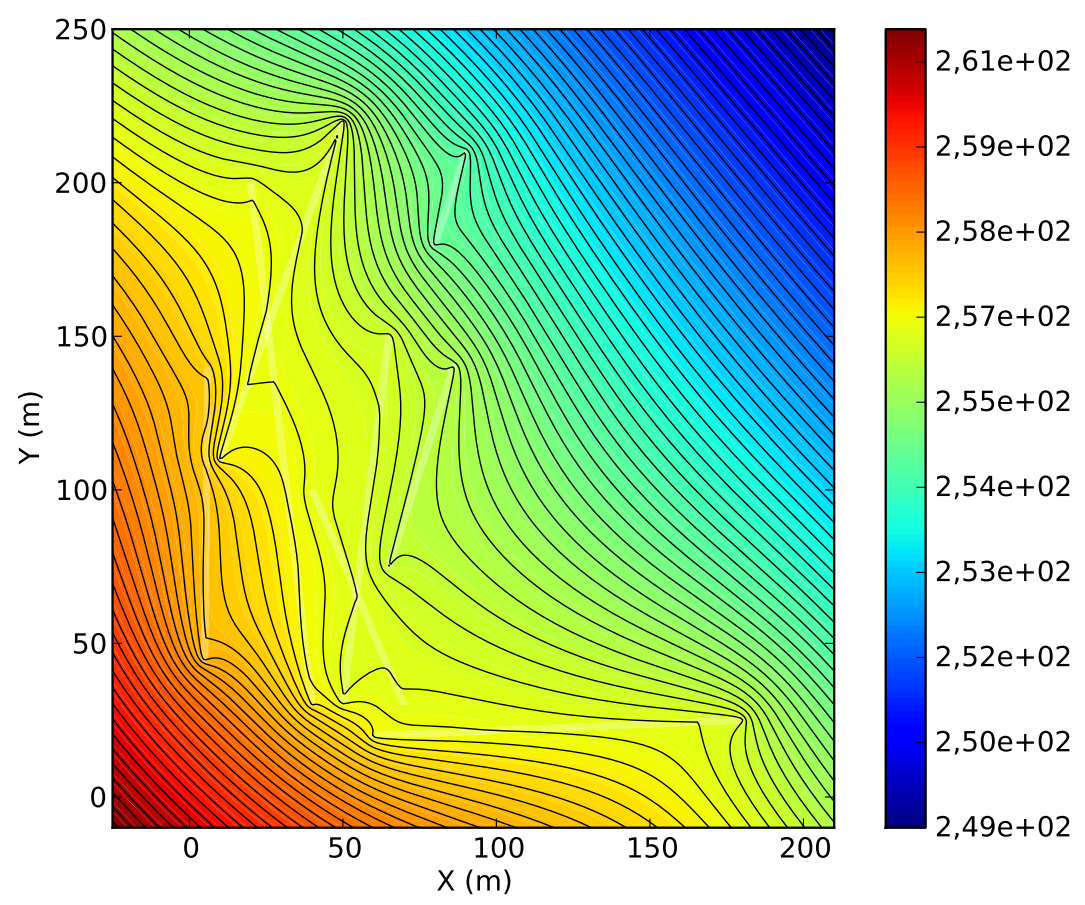

Figura 7.17 - Sistema de fraturas em cruzamento em um campo de escoamento uniforme a $\pi / 4$ radianos. Os parâmetros estão relacionados na tabela (7.11). Valores de carga hidráulica.

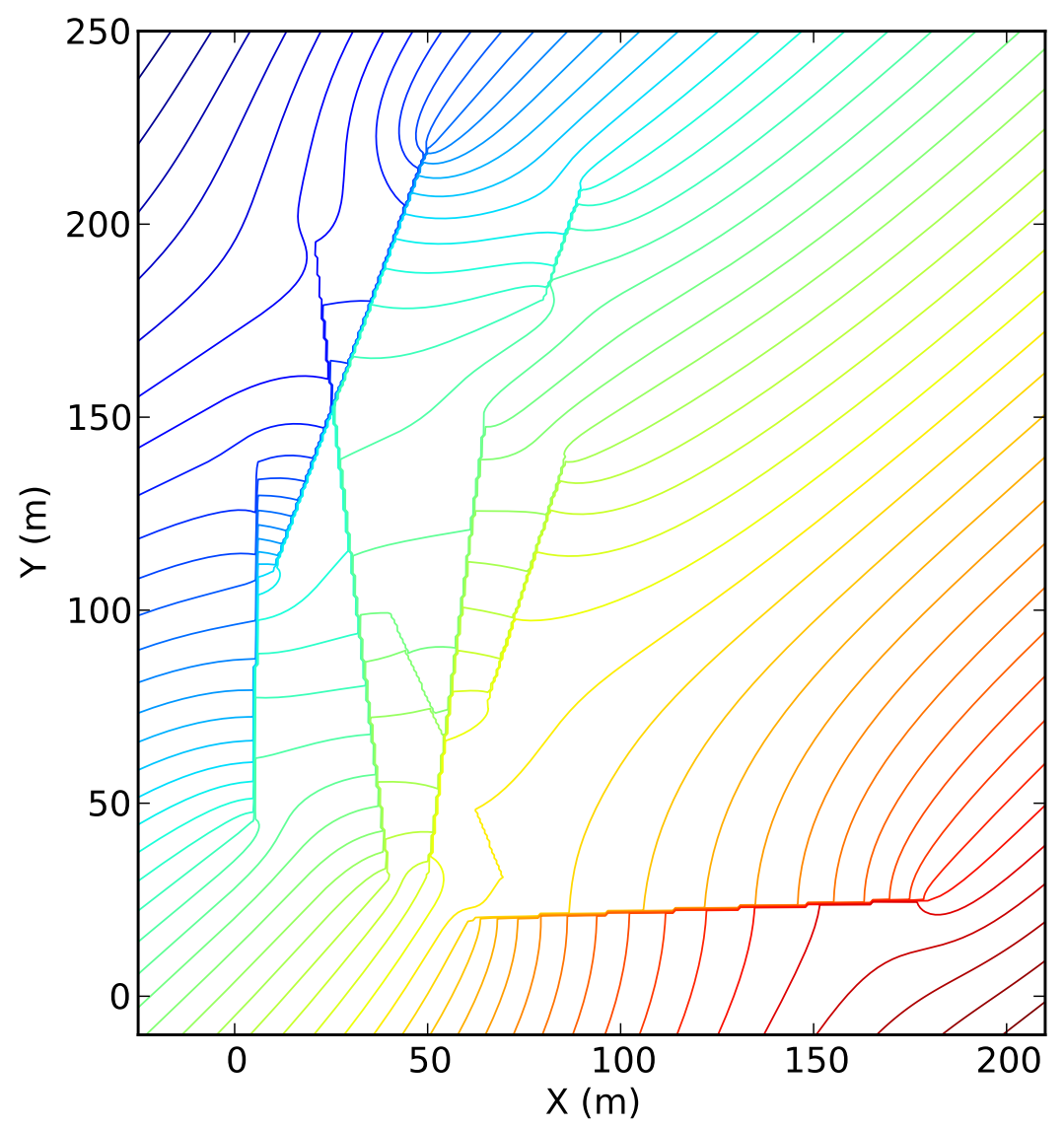

Figura 7.18 - Sistema de fraturas em cruzamento em um campo de escoamento uniforme a $\pi / 4$ radianos. Linhas de corrente. Os parâmetros estão relacionados na tabela (7.11). 
Sistema de fraturas com cruzamento de carga e variação na abertura e condutividade hidráulica. Cada fratura tem o seu valor de abertura e carga hidráulica dada pela lei cúbica (3.5.1), relacionados na tabela (7.12). A geometria do conjunto de fraturas é a mesma da figura (7.17).

Tabela 7.12 - Parâmetros para a simulação do sistema fraturado com variação na abertura e na condutividade hidráulica, com valores calculados de acordo com a lei cúbica.

\begin{tabular}{|c|c|c|c|}
\hline & $K^{+}(M / s)$ & $b(m)$ & $n$ \\
\hline Fratura 1 & 0,000074 & 0,0003 & 5 \\
Fratura 2 & 0,000033 & 0,0002 & 5 \\
Fratura 3 & 0,0002 & 0,0005 & 5 \\
Fratura 4 & 0,000033 & 0,0002 & 5 \\
Fratura 5 & 0,000002 & 0,00005 & 5 \\
Fratura 5 & 0,000013 & 0,0004 & 5 \\
Fratura 6 & 0,0002 & 0,0004 & 5 \\
Fratura 7 & 0,00029 & 0,0006 & 5 \\
Fratura 8 & 0,00053 & 0,0008 & 5 \\
\hline
\end{tabular}

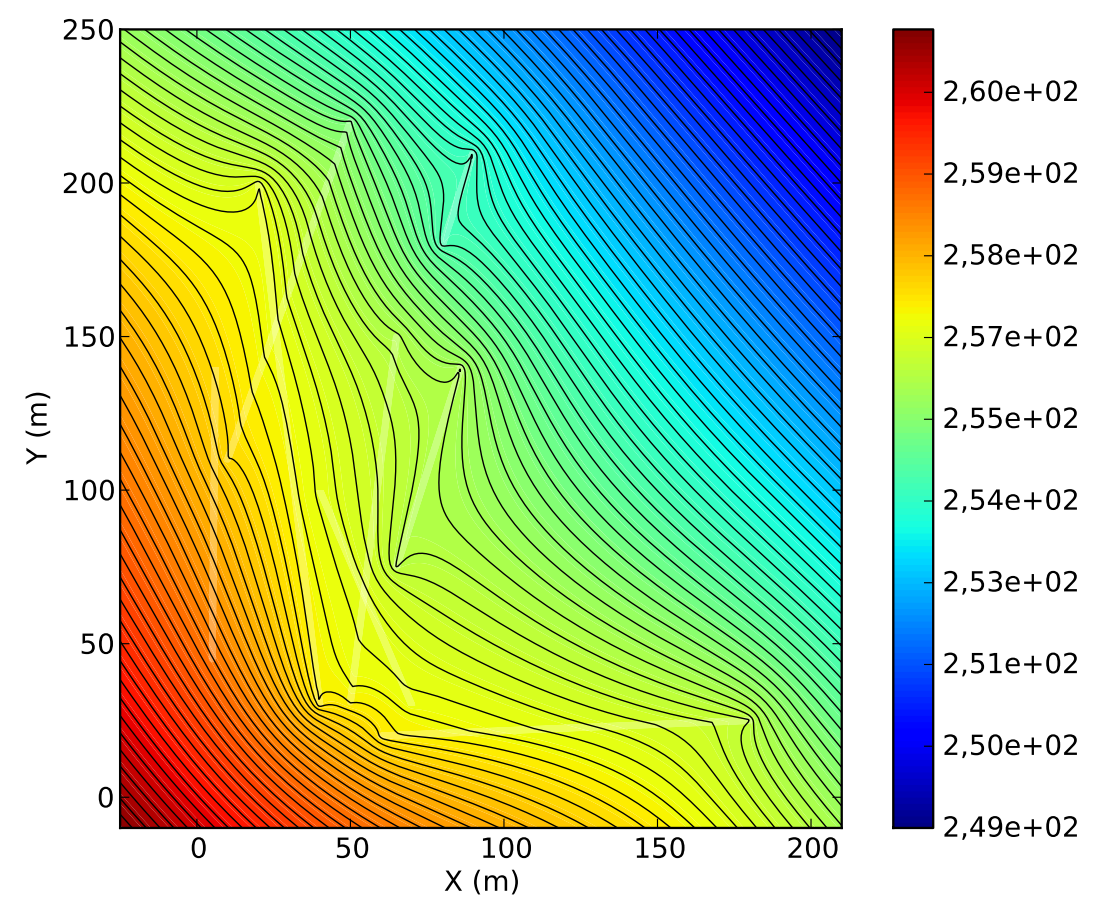

Figura 7.19 - Sistema de fraturas em cruzamento em um campo de escoamento uniforme a $\pi / 4$ radianos. Valores de carga hidráulica.

A figura (7.21) combina as isolinhas de carga hidráulica e as linhas de corrente na mesma figura. As isolinhas de carga hidráulica são as linhas cheias e as linhas pontilhadas são as linhas de corrente. Como esperado, as linhas de corrente são perpendiculares às isolinhas de carga. Observa-se que fraturas com aberturas e condutividades hidráulicas pequenas, como a da extrema esquerda, 


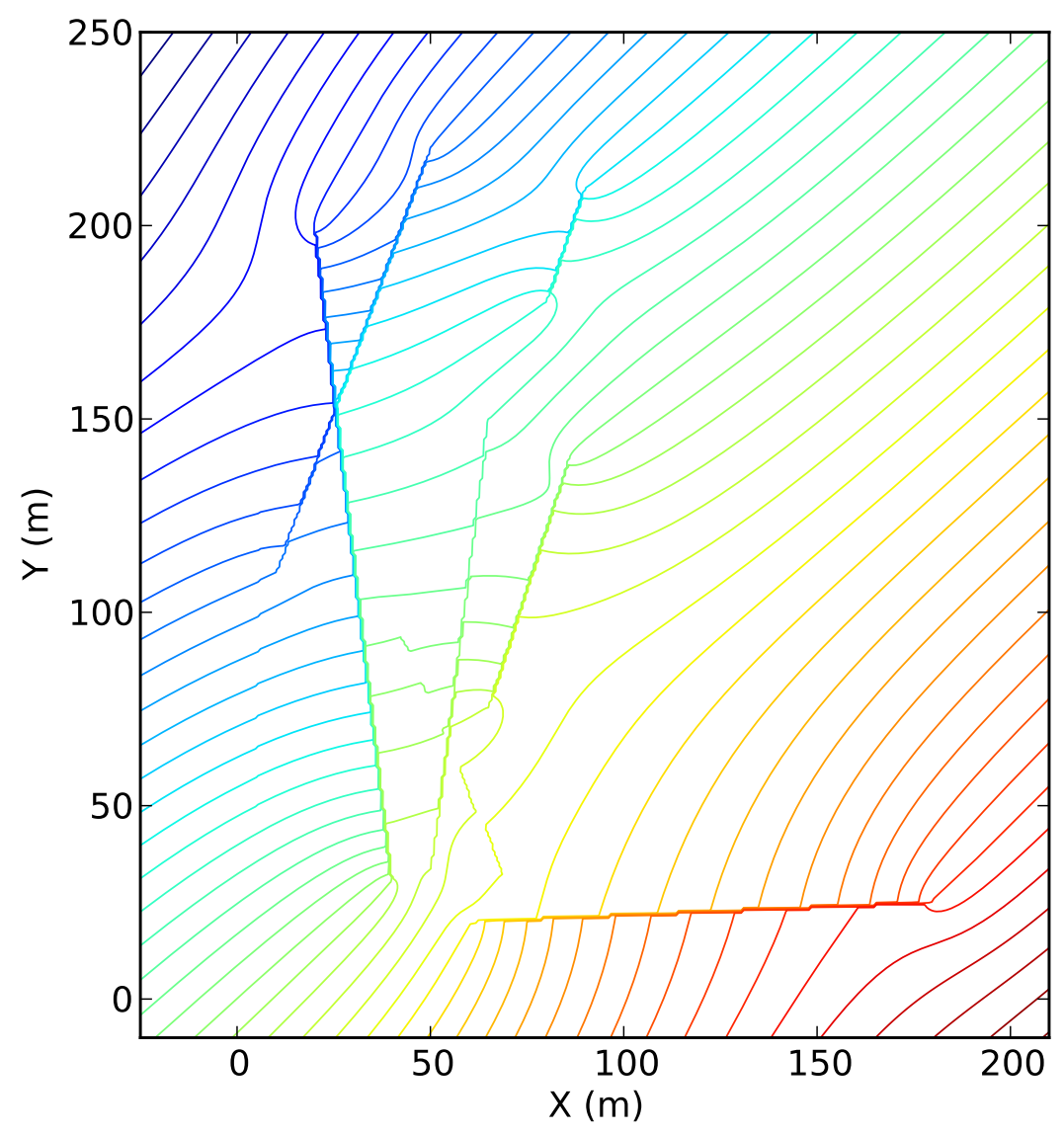

Figura 7.20 - Sistema de fraturas em cruzamento em um campo de escoamento uniforme a $\pi / 4$ radianos. Linhas de corrente.

influcienciam o escoamento de forma marginal, enquanto fraturas com condutividade hidráulica e abertura maiores têm maior influência. 


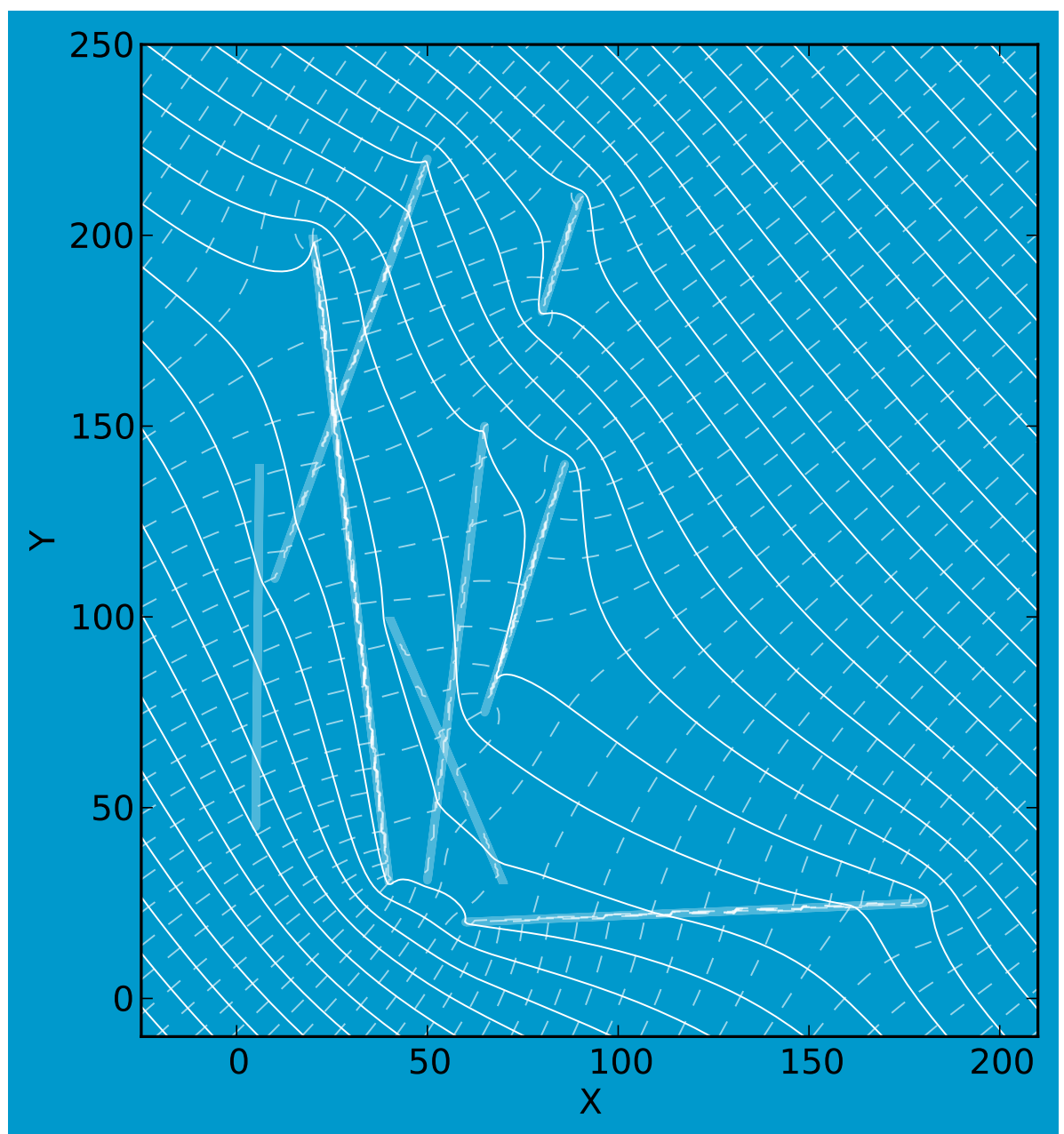

Figura 7.21 - Sistema de fraturas em cruzamento em um campo de escoamento uniforme a $\pi / 4$ radianos. Isolinhas de carga hidráulica e linhas de corrente, com os parâmetros dados pela tabela (7.12). 


\section{Conclusões e Perspectivas Futuras}

Este trabalho propôs simular o escoamento de água subterrânea em meio fraturado usando o Método de Elementos Analíticos. Durante seu desenvolvimento, problemas de convergência para para o método iterativo implícito usado por Strack (48) levaram ao desenvolvimento de uma abordagem matricial. Foi possível neste trabalho desenvolver o elemento analítico para uma formação rochosa fraturada, com parâmetros reais. Os desenvolvimentos desse trabalho, o elemento analítico para um sistema de fraturas, e o método matricial para encontrar os coeficientes desconhecidos na expansão em série dos elementos para satisfazer as condições de contorno são inéditos na literatura, principalmente no contexto do MEA. A implementação em Python, apesar de não ser a estratégia mais eficiente, demonstra a flexibilidade do elemento analítico para fratura na implementação e a capacidade de se usar o mesmo método de cálculo de coeficientes desconhecidos em expansão em série para outros elementos. O método matricial também apresenta inovação, já que, usando resultados conhecidos na literatura, apresenta a capacidade de resolver sistemas lineares, conjuntamente com as integrais de Cauchy, que anteriormente apresentavam divergência. As conclusões deste trabalho são apresentadas abaixo.

\subsection{Inomogeneidades Circulares}

As inomogeneidades circulares foram simuladas usando o formalismo iterativo implícito, matricial e iterativo explícito. A concordância da solução numérica com a solução exata foi excelente, com erro relativo menor que a precisão numérica de máquina. O potencial complexo para inomogeneidades circulares é conhecido (93), assim como a forma de solução iterativa implícita, mas a aplicação do método matricial com integração numérica não foi encontrado na literatura ${ }^{1}$. A simulação de diversas inomogeneidades circulares com raios e condutividades hidráulicas diferentes também foi demonstrada. A implementação e a solução foi comparada com a literatura existente, reproduzindo os resultados de (93). O custo computacional do método iterativo implícito é maior em tempo de processamento e menor em uso de memória, enquanto no método matricial o inverso ocorre. Os resultados obtidos com as inomogeneidades circulares serviram como base para a comparação e verificação das inomogeneidades poligonais formadas por line doublets.

\footnotetext{
${ }^{1}$ Não tive acesso ao trabalho de Salisbury, que ao que tudo indica aplicou este método para inomogeneidades circulares de acordo com (93).
} 


\subsection{Inomogeneidades Poligonais formadas por Line Dou- blets}

As inomogeneidades poligonais foram implementadas usando o elemento analítico line doublet. O formalismo para a construção do potencial complexo para a line doublet é proveniente de (48) e a implementação para o algoritmo iterativo implícito foi baseado nessa referência. O método matricial desenvolvido nesse trabalho e o algoritmo iterativo explícito foram aplicados para esse elemento com resultados satisfatórios, demostrando a aplicabilidade do método matricial para elementos analíticos e da nova forma do potencial complexo para line doublets no plano $\chi$. A comparação entre o método iterativo implícito e o método matricial mostrou a boa concordância entre ambos e sua equivalência na solução. O método iterativo implícito, na implementação feita neste trabalho, apresentou problemas de convergência, levando em alguns casos até 800 iterações para convergência, dependendo da diferença de condutividade hidráulica entre a inomogeneidade poligonal e a de fundo. O método matricial, por outro lado, por não envolver iteração, apresentou um tempo de processamento para montagem da matriz mais elevado, mas para todos os sistemas testados o tempo de solução do sistema linear foi mínimo, comparado com a montagem da matriz. O custo em memória para o método matricial, apesar de maior que no caso do método iterativo implícito, ainda foi muito menor que a memória total em um computador de mesa.

As line doublets, além de fornecerem uma base para testes e validação dos métodos de solução, serviu também para a comparação com a solução proposta para fraturas.

\subsection{Fraturas}

O potencial complexo para uma fratura formada a partir do deslocamento elástico de um meio infinito foi obtida a partir de (4). Este potencial complexo para uma fratura foi analisado e implementado. A solução obtida foi comparada com uma inomogeneidade elipsóide poligonal usando line doublets, com boa concordância para aberturas pequenas em relação ao comprimento.

O potencial complexo para um sistema de fraturas discretas em meio poroso foi desenvolvido neste trabalho, baseado no potencial exato para uma fratura. Os métodos de solução usados para line doublets, a integração de Cauchy no plano $\chi$, desenvolvidos por (48) foram aplicados. Entretanto, convergência numérica foi obtida para poucos casos ao se usar o algoritmo iterativo implícito. Esse fato levou ao desenvolvimento de um método matricial, a partir da transformação do método iterativo implícito. O método matricial conseguiu fornecer soluções para o caso da fratura, e a comparação entre o sistema de fraturas e o sistema de inomogeneidades elipsóides poligonais mostrou boa concordância. As diferenças entre as fraturas e as inomogeneidades poligonais provém da descrição aproximada da abertura para a fratura, nas condições de contorno, mais tais erros foram considerados pequenos devido à relação entre abertura e comprimento da fratura.

O custo computacional para as fraturas é consideravelmente menor que para as line doublets, já que cada fratura é representada por um elemento analítico para fratura, e 28 line doublets para a 
inomogeneidade poligonal equivalente ótima. A maior parte do tempo de processamento é gasta na construção da matriz, enquanto a solução do sistema linear é praticamente instantânea para os casos estudados. O uso de memória foi muito menor que o total de memória de um computador de mesa, mesmo quando o sistema de 6 fraturas foi simulado, onde foram usados 6 elementos de fratura e 168 line doublets para comparação.

O escoamento em um sistema com cruzamento de fraturas foi simulado, com resultados aparentemente consistentes com o esperado. Entretanto, a condição de contorno no cruzamento, a conservação do vetor de descarga, não foi incluída no potencial complexo de forma explícita. Como o potencial complexo das line doublets não permite o cruzamento das mesmas, não foi possível comparar com inomogeneidades poligonais.

\subsection{O Método Matricial}

Devido às dificuldades encontradas com o algoritmo iterativo implícito no caso de fraturas, o método matricial foi desenvolvido neste trabalho. O método é baseado no método iterativo implícito, mas descarta a iteração e constrói um sistema linear que deve ser resolvido somente uma vez. O método matricial aproveita-se dos resultados da integração de Cauchy no plano $\chi$, usando a ortogonalidade das funções de base da expansão dos elementos analíticos para a construção da matriz. A concordância entre o método iterativo implícito e o método matricial foi boa, mostrando a equivalência de ambas.

A divergência do método iterativo também motivou o estudo do comportamento das soluções iterativas para os problemas propostos, com duas partições iterativas explícitas sendo estudadas, a partição de Jacobi e a partição de Gauss-Seidel. O sistema iterativo implícito é semelhante à uma iteração de Gauss-Seidel, apesar de apresentar diferenças. O raio espectral do sistema iterativo explícito foi analisado, e foram observados casos onde o raio espectral é maior que 1 , o que indica que a convergência do sistema para um vetor de soluções inicial não é garantida; e mesmo que haja convergência, a mesma pode ser lenta, como foi verificado em alguns sistemas simulados.

No desenvolvimento do método matricial foi notada a semelhança entre a descrição das condições de contorno como mínimos quadrados e exata, e a equivalência para o caso dos elementos descritos entre o método de mínimos quadrados formando uma matriz quadrada e a integração numérica usando a regra do trapézio, fato notado para o caso das inomogeneidades circulares em (93).

Como é comum em simulação numérica e em computação em geral, existe um compromisso entre uso de memória e tempo de processamento. Um uso maior da memória leva a um menor custo em processamento, e vice-versa. Esta relação foi observada na comparação da solução do mesmo problema para o método iterativo implícito e o método matricial. O custo computacional da integração numérica usada no caso matricial poderia ser diminuído, se outras técnicas de integração numérica fossem aplicados. Porém o ganho no tempo de processamento não foi significativamente maior com o uso de técnicas de integração numérica mais rápidas, como a regra do trapézio, com prejuízo na precisão da solução. Um estudo mais aprofundado das integrais e do método de solução 
das mesmas é um dos trabalhos futuros a serem desenvolvidos.

\subsection{Programação Orientada a Objetos e o MEA}

Alencar Neto (55) apresenta extensivamente os argumentos para aplicação de orientação a objetos ao Método de Elementos Analíticos. A separação entre as características geométricas e de influência, juntamente com o tratamento de cada elemento de forma independente, forma uma estrutura coerente para a aplicação de uma estrutura orientada a objetos no MEA. Bakker e Kelson (102) seguem a mesma linha, apresentando, inclusive, um exemplo de uma estrutura geral usando herança.

Uma primeira abordagem foi feita nestas linhas, em $\mathrm{C}++$, sem muito sucesso. As restrições de acesso aos membros dos objetos, juntamente com as interdependências entre variáveis - como a condutividade hidráulica pertencente à cada line doublet para calcular o potencial total de uma inomogeneidade poligonal - preveniram um desacoplamento total entre as duas partes, tornando a implementação complexa.

$\mathrm{Na}$ implementação do presente trabalho em Python houve um maior foco na funcionalidade do código do que em sua estrutura, violando alguns preceitos de uma definição de orientação a objetos, como a ausência de herança e polimorfismo. Foram definidas classes para cada elemento e para cada solver, mas sem a preocupação em manter uma estrutura de orientação a objetos rígida. Apesar dos problemas inerentes que a falta de estrutura pode trazer, como maior dificuldade na extensão do código e em sua documentação, a mesma razão pela escolha do Python - maior facilidade e flexibilidade na implementação - ditaram as decisões na hora da definição da estrutura. Também não houveram esforços no sentido de otimização ou melhoria de performance, área que pode ser muito melhorada. Certas propriedades das matrizes envolvidas e das operações realizadas podem permitir ganhos de desempenho, o que tornaria a implementação em Python ainda mais atrativa.

Python apresenta ainda mais uma vantagem em relação a linguagens compiladas: portabilidade. Um código escrito em Python é muito mais portável e de mais fácil distribuição, já que na máquina destino só é necessário haver um ambiente Python instalado, ao contrário de um pacote binário, que precisa ser compilado para cada plataforma e muitas vezes precisa incluir bibliotecas, o que o torna grande.

Todo o software produzido neste trabalho foi desenvolvido usando ferramentas open source, em ambiente Linux (distribuição Debian (104)). O código, tanto em C++ quanto em Python, foi desenvolvido usando a interface de desenvolvimento Eclipse (105).

\subsection{Perspectivas Futuras}

O Método de Elementos Analíticos, apesar de restrito à área de água subterrânea, apresenta características que as outras técnicas de solução de equações diferenciais, como elementos finitos, não 
são capazes de reproduzir. Por não necessitar de discretização do domínio, o MEA apresenta-se como um bom candidato para a solução de problemas nos quais fenômenos em diferentes escalas interagem e contribuem para a solução final. A solução apresentada para a fratura neste trabalho ainda pode ser melhorada, incluindo outros fenômenos que influenciam o escoamento em sistemas fraturados, como rugosidade e tortuosidade. O cruzamento de fraturas também é uma outra área onde mais estudos são requeridos, principalmente na verificação de que o cruzamento das fraturas conserva o vetor descarga, dado que o cruzamento e a interconexão de fraturas é um ponto importante no estudo do escoamento em sistemas fraturados, principalmente ao se considerar aspectos de transporte de solutos.

A integração com outros elementos analíticos na mesma simulação é outra área onde este trabalho pode ser melhorado. Com os potenciais complexos já determinados, a integração entre diversos elementos é mais um problema de implementação computacional do que de desenvolvimento teórico, apesar de que certos cuidados com as condições de contorno, como a ocorrência de cruzamentos, precisam ser melhor entendidos.

Os aspectos numéricos levantados neste trabalho podem ser aprofundados, principalmente pela falta na literatura do MEA de estudos para os métodos de solução gerais. O uso do raio espectral pode ser considerado como um primeiro passo no estudo da convergência geral dos métodos iterativos aplicados ao MEA. Uma análise mais detalhada da propagação de erro numérico também é uma área a ser considerada para estudos futuros.

Soluções de escoamento do MEA já foram usadas na literatura como parâmetros de entrada para simulações de transporte de solutos $(106,107)$. Com a relevância do estudo de transporte de solutos para repositórios geológicos e os domínios muitas vezes em escala regional, o uso do elemento desenvolvido neste trabalho na integração com modelos de transporte mostra-se como uma proposta interessante. Novos avanços do MEA, como condutividade hidráulica variando de forma estratificada (108), também podem ser aplicados. A integração do MEA com outras abordagens numéricas, como diferenças finitas (109), mostra a versatilidade do método na solução dos mais diversos problemas em águas subterrâneas. 


\section{Referências Bibliográficas}

1 STRACK, O. D. L. Groundwater Mechanics. EUA: Strack Consulting Inc., 1999.

2 KLIMCZAK, C.; SCHULTZ, R.; PARASHAR, R.; REEVES, D. Cubic law with aperture-length correlation: implications for network scale fluid flow. Hydrogeology Journal, v. 18, n. 4, p. 851-862, jun. 2010. Disponível em: <http://dx.doi.org/10.1007/s10040-009-0572-6>.

3 CRANDALL, D.; AHMADI, G.; SMITH, D. H. Computational modeling of fluid flow through a fracture in permeable rock. Transport in Porous Media, v. 84, n. 2, p. 493-510, 2010. ISSN 0169-3913. Disponível em: <http://www.springerlink.com.floyd.lib.umn.edu/content/8430t042j6gqx086/>.

4 STRACK, O. D. L. Analytic Modeling of Flow in a Permeable Fissured Medium. EUA, February 1982. Assessment of Effectiveness of Geologic Isolation System.

5 ROBINS, N.; ROSE, E. Military uses of groundwater: a driver of innovation? Hydrogeology Journal, 2009. Disponível em: <http://dx.doi.org/10.1007/s10040-008-0428-5>.

6 FARMER, J. Data drought, data flood. Science, v. 323, n. 5913, p. 462-463, jan. 2009. Disponível em: <http://www.sciencemag.org $>$.

7 GALLOWAY, D. The complex future of hydrogeology. Hydrogeology Journal, v. 18, n. 4, p. 807-810, jun. 2010. Disponível em: <http://dx.doi.org/10.1007/s10040-010-0585-1>.

8 GREEN, T. R.; TANIGUCHI, M.; KOOI, H.; GURDAK, J. J.; ALLEN, D. M.; HISCOCK, K. M.; TREIDEL, H.; AURELI, A. Beneath the surface of global change: Impacts of climate change on groundwater. Journal of Hydrology, In Press, Accepted Manuscript, 2009. ISSN 0022-1694. Disponível em: <http://www.sciencedirect.com/science/article/B6V6C-52T77YD2/2/166ea3c151f1edc47f561f6917e79743>.

9 BROOK, E. Palaeoclimate: Windows on the greenhouse. Nature, v. 453, n. 7193, p. 291-292, maio 2008. ISSN 0028-0836. Disponível em: <http://dx.doi.org/10.1038/453291a>.

10 ROSENZWEIG, C.; KAROLY, D.; VICARELLI, M.; NEOFOTIS, P.; WU, Q.; CASASSA, G.; MENZEL, A.; ROOT, T. L.; ESTRELLA, N.; SEGUIN, B.; TRYJANOWSKI, P; LIU, C.; RAWLINS, S.; IMESON, A. Attributing physical and biological impacts to anthropogenic climate change. Nature, v. 453, n. 7193, p. 353-357, maio 2008. ISSN 0028-0836. Disponível em: <http://dx.doi.org/10.1038/nature06937>.

11 EDITORIAL. The next big climate challenge. Nature, v. 453, n. 7193, p. 257, maio 2008. ISSN 0028-0836. Disponível em: <http://dx.doi.org/10.1038/453257a>.

12 BRUMFIEL, G. The meltdown that wasn't. Nature, v. 471, n. 7339, p. 417-418, mar. 2011. ISSN 0028-0836. Disponível em: <http://www.nature.com/doifinder/10.1038/471417a>.

13 BUTLER, D. Energy: Nuclear power's new dawn. Nature, v. 429, n. 6989, p. 238-240, maio 2004. ISSN 0028-0836. Disponível em: <http://dx.doi.org/10.1038/429238a>.

14 FERGUSON, C. Do not phase out nuclear power... yet. Nature, v. 471, n. 7339, p. 411-411, mar. 2011. ISSN 0028-0836. Disponível em: <http://www.nature.com/doifinder/10.1038/471411a>. 
15 BUTLER, D. France digs deep for nuclear waste. Nature, v. 466, p. 804-805, ago. 2010. Disponível em: <http://www.nature.com/news/2010/100810/full/466804a.html>.

16 ZAKAIB, G. D. Radiation risks unknown. Nature, v. 471, n. 7339, p. 419-419, mar. 2011. ISSN 0028-0836. Disponível em: <http://www.nature.com/doifinder/10.1038/471419a>.

17 HAMILTON, L.; SCOWCROFT, B. Nuclear waste: Progress with public engagement. Science, v. 330, n. 6003, p. 448-a, out. 2010. Disponível em: <http://www.sciencemag.org>.

18 MACILWAIN, C. Concerns over nuclear energy are legitimate. Nature, v. 471, n. 7340, p. 549-549, mar. 2011. ISSN 0028-0836. Disponível em: <http://www.nature.com/doifinder/10.1038/471549a>.

19 ROSA, E. A.; TULER, S. P.; FISCHHOFF, B.; WEBLER, T.; FRIEDMAN, S. M.; SCLOVE, R. E.; Shrader-Frechette, K.; ENGLISH, M. R.; KASPERSON, R. E.; GOBLE, R. L.; LESCHINE, T. M.; FREUDENBURG, W.; CHESS, C.; PERROW, C.; ERIKSON, K.; SHORT, J. F. Nuclear waste: Progress with public Engagement-Response. Science, v. 330, n. 6003, p. 448-b-449, out. 2010. Disponível em: $<$ http://www.sciencemag.org $>$.

20 RADIATION, U. N. S. C. on the Effects of A. Optimizing the international effort to study, mitigate and minimize the consequences of the Chernobyl disaster. September 2010. Disponível em: <http://www.unscear.org/unscear/en/chernobyl.html>.

21 WILLIAMS, D.; BAVERSTOCK, K. Chernobyl and the future: Too soon for a final diagnosis. Nature, v. 440, n. 7087, p. 993-994, abr. 2006. ISSN 0028-0836. Disponível em: $<$ http://dx.doi.org/10.1038/440993a>.

22 AGENCY, I. A. E. Nuclear Power Reactors in the World. 2010. Disponível em: <http://wwwpub.iaea.org/MTCD/publications/PDF/iaea-rds-2-30_web.pdf>.

23 WITHERSPOON, P.; COOK, N.; GALE, J. Geologic storage of radioactive waste: field studies in sweden. Science, v. 211, n. 4485, p. 894-900, fev. 1981. Disponível em: <http://www.sciencemag.org/cgi/content/abstract/211/4485/894>.

24 WITHERSPOON, P. A.; NELSON, P.; DOE, T.; THORPE, R.; PAULSSON, B.; GALE, J.; FORSTER, C. Rock mass characterization for storage of nuclear waste in granite. Nuclear Science, IEEE Transactions on, v. 27, n. 4, p. 1280-1290, 1980. ISSN 0018-9499.

25 BREDEHOEFT, J. D.; MAINI, T. Strategy for radioactive waste disposal in crystalline rocks. Science, v. 213, n. 4505, p. 293-296, jul. 1981. Disponível em: <http://www.sciencemag.org/cgi/content/abstract/213/4505/293>.

26 BEAR, J.; TSANG, C.; MARSILY, G. de. Flow and Contaminant Transport in Fractured Rock. EUA: Academic Press, 1993.

27 COMMITTEE, A. C. R.; HEBEL, L. C.; CHRISTENSEN, E. L.; DONATH, F. A.; FALCONER, W. E.; LIDOFSKY, L. J.; MONIZ, E. J.; MOSS, T. H.; PIGFORD, R. L.; PIGFORD, T. H.; ROCHLIN, G. I.; SILSBEE, R. H.; WRENN, M. E.; FRAUENFELDER, H.; CAIRNS, T. L.; PANOFSKY, W. K. H.; SIMMONS, M. G. Report to the american physical society by the study group on nuclear fuel cycles and waste management. Reviews of Modern Physics, v. 50, n. 1, p. S1, jan. 1978. Disponível em: $<$ http://link.aps.org/doi/10.1103/RevModPhys.50.S1>.

28 WALKER, D.; GYLLING; ANDERS; SELROOS, J. Hydrogeologic studies for nuclear-waste disposal in sweden. Hydrogeology Journal, v. 9, n. 5, p. 419-431, 2001. ISSN 1431-2174. Disponível em: $<$ http://www.springerlink.com/openurl.asp?genre=article\&id=doi:10.1007/s100400100156>. 
29 MARSILY, G. de; LEDOUX, E.; BARBREAU, A.; MARGAT, J. Nuclear waste disposal: can the geologist guarantee isolation? Science, v. 197, p. 519, 1977.

30 U.S. Department of Energy Waste Isolation Pilot Plant. Http://www.wipp.energy.gov/. Disponível em: <http://www.wipp.energy.gov/>.

31 BAKKER, M.; ANDERSON, E. I.; OLSTHOORN, T. N.; STRACK, O. D. L. Regional groundwater modeling of the yucca mountain site using analytic elements. Journal of Hydrology, v. 226, n. 3-4, p. 167-178, dez. 1999. Disponível em: <http://www.sciencedirect.com/science/article/B6V6C3YHFYKY-6/1/afb23ba922e5e59b53120e3da72e7718>.

32 BERKOWITZ, B. Characterizing flow and transport in fractured geological media: A review. Advances in Water Resources, v. 25, n. 8-12, p. 861-884, 2002. ISSN 0309-1708. Disponível em: <http://www.sciencedirect.com/science/article/B6VCF-478B4BX1/2/81da2717a9971fdfc480ca955d45da14>.

33 NEUMAN, S. P. Trends, prospects and challenges in quantifying flow and transport through fractured rocks. Hydrogeology Journal, v. 13, n. 1, p. 124-147, mar. 2005. Disponível em: <http://dx.doi.org/10.1007/s10040-004-0397-2>.

34 WEATHERILL, D.; GRAF, T.; SIMMONS, C. T.; COOK, P. G.; THERRIEN, R.; REYNOLDS, D. a. Discretizing the Fracture-Matrix interface to simulate solute transport. Ground Water, v. 0, n. 0, p. ???, 2008. Disponível em: <http://www.blackwell-synergy.com/doi/abs/10.1111/j.17456584.2007.00430.x>.

35 MADADI, M.; SAHIMI, M. Lattice boltzmann simulation of fluid flow in fracture networks with rough, self-affine surfaces. Physical Review E, v. 67, n. 2, p. 026309, fev. 2003. Copyright (C) 2008 The American Physical Society; Please report any problems to prola@aps.org. Disponível em: <http://link.aps.org/abstract/PRE/v67/e026309>.

36 MORRIS, J. P.; FOX, P. J.; ZHU, Y. Modeling low reynolds number incompressible flows using SPH. Journal of Computational Physics, v. 136, n. 1, p. 214-226, set. 1997. ISSN 0021-9991. Disponível em: <http://www.sciencedirect.com/science/article/B6WHY-45V7FSXG/2/43b922e2a2ca81607847cf038c3afe49>.

37 GRAND, P. L. Advanced Curvilinear Shapes for Object Centering Modeling of Groundwater Flow with the Analytic Element Method. Tese (Doutorado) - Ecole Nationale Supériere des Mines - Saint Etienne, 2003.

38 STRACK, O. D. L. Principles of the analytic element method. Journal of Hydrology, v. 226, n. $3-4$, p. 128-138, dez. 1999. ISSN 0022-1694. Disponível em: <http://www.sciencedirect.com/science/article/B6V6C-3YHFYKY2/2/abacb51d1130f4dc69fbb8327f65b004>.

39 BAKKER, M. Derivation and relative performance of strings of line elements for modeling (un)confined and semi-confined flow. Advances in Water Resources, v. 31, n. 6, p. 906914, jun. 2008. Disponível em: <http://www.sciencedirect.com/science/article/B6VCF-4S0PKNG2/1/f62af25c62ba97b380faa716b51a2e0a>.

40 HUNT, R. J. Ground water modeling applications using the analytic element method. Ground Water, v. 44, n. 1, p. 5-15, jan. 2006. Disponível em: <http://www.blackwellsynergy.com/doi/abs/10.1111/j.1745-6584.2005.00143.x>.

41 LANGE, W. J. D. Development of an analytic element ground water model of the netherlands. Ground Water, v. 44, n. 1, p. 111-115, jan. 2006. Disponível em: <http://www.blackwellsynergy.com/doi/abs/10.1111/j.1745-6584.2005.00142.x>. 
42 HAITJEMA, H. M.; HUNT, R. J.; JANKOVIC, I.; LANGE, W. J. D. Foreword: Ground water flow modeling with the analytic element method. Ground Water, v. 44, n. 1, p. 1-2, jan. 2006. Disponível em: <http://www.blackwell-synergy.com/doi/abs/10.1111/j.1745-6584.2005.00144.x>.

43 KRAEMER, S. R. Analytic element ground water modeling as a research program (1980 to 2006). Ground Water, v. 45, n. 4, p. 402-408, 2007. Disponível em: <http://dx.doi.org/10.1111/j.17456584.2007.00314.x>.

44 RAYMOND, H. a.; BONDOC, M.; MCGINNIS, J.; METROPULOS, K.; HEIDER, P; REED, A.; SAINES, S. Using analytic element models to delineate drinking water source protection areas. Ground Water, v. 44, n. 1, p. 16-23, jan. 2006. Disponível em: <http://www.blackwellsynergy.com/doi/abs/10.1111/j.1745-6584.2005.00122.x>.

45 STRACK, O. The generating analytic element approach with application to the modified helmholtz equation. Journal of Engineering Mathematics, v. 64, n. 2, p. 163-191, jun. 2009. Disponível em: $<$ http://dx.doi.org/10.1007/s10665-009-9279-x>.

46 CRAIG, J. R. Analytic elements for flow in harmonically heterogeneous aquifers. jun. 2009. Disponível em: <http://www.agu.org/pubs/crossref/2009/2009WR007800.shtml>.

47 STRACK, O. The development of new analytic elements for transient flow and multiaquifer flow. Ground Water, v. 44, n. 1, p. 91-98, jan. 2006. Disponível em: <http://www.blackwellsynergy.com/doi/abs/10.1111/j.1745-6584.2005.00148.x>.

48 STRACK, O. D. L. AEM Method, in preparation. EUA: [s.n.], 2011.

49 TUCCI, C. E. M. Hidrologia Ciência e Aplicação. Brasil: UFRGS Editora/ABRH, 2002.

50 FITTS, C. R. Groundwater Science. EUA: Academic Press, 2002.

51 BATISTA, J. A. do N. Modelagem de Escoamento em Aquíferos Longos Baseada no Método de Elementos Analíticos. Tese (Doutorado) — Universidade de São Paulo, 2006.

52 BEAR, J. Dynamics of Fluids in Porous Media. EUA: Dover Publications, 1988.

53 WHITE, F. M. Fluid Mechanics. EUA: [s.n.], 2006.

54 LANDAU, L. D.; LIFSHITZ, E. M. Statistical Physics. Rússia: Pergamon, 1980.

55 NETO, M. da F. A. Interpolação por Splines para Modelação de Inomogeneidades no Método de Elementos Analíticos - Implementação por Programação Orientada a Objetos. Tese (Doutorado) Departamento de Hidráulica e Saneamento, Escola de Engenharia de São Carlos, Universidade de São Paulo, 2008.

56 WENDLAND, E.; SCHMID, G. 6th International Conference on Computing in Civil and Building Engineering. Alemanha: Balkema, 1995.

57 PLUIJM, B. A. van der; MARSHAK, S. Earth Structure. EUA: W. W. Norton Company, 2004.

58 BONNET, E.; BOUR, O.; ODLING, N. E.; DAVY, P; MAIN, I.; COWIE, P.; BERKOWITZ, B. Scaling of fracture systems in geological media. Reviews of Geophysics, v. 39, n. 3, p. PP. 347-383, 2001. Disponível em: <http://www.agu.org.floyd.lib.umn.edu/journals/rg/v039/i003/1999RG000074/>.

59 BERKOWITZ, B.; SCHER, H. Theory of anomalous chemical transport in random fracture networks. Physical Review E, v. 57, n. 5, p. 5858, maio 1998. Disponível em: $<$ http://link.aps.org/doi/10.1103/PhysRevE.57.5858>. 
60 WENDLAND, E.; HIMMELSBACH, T. Transport simulation with stochastic aperture for a single fracture - comparison with a laboratory experiment. Advances in Water Resources, v. 25, n. 1, p. 19-32, jan. 2002. Disponível em: < http://www.sciencedirect.com/science/article/B6VCF-44PK3KB2/1/8861ed09ec5dc46fd447ba45fd175da8>.

61 DAVY, P.; GOC, R. L.; DARCEL, C.; BOUR, O.; DREUZY, J. R. d.; MUNIER, R. A likely universal model of fracture scaling and its consequence for crustal hydromechanics. Journal of Geophysical Research, v. 115, p. 13 PP., out. 2010. Disponível em: $<$ http://www.agu.org/pubs/crossref/2010/2009JB007043.shtml>.

62 BAE, D.-s.; KIM, K.-s.; KOH, Y.-k.; KIM, J.-y. Characterization of joint roughness in granite by applying the scan circle technique to images from a borehole televiewer. Rock Mechanics and Rock Engineering, v. 44, n. 4, p. 497-504, fev. 2011. ISSN 0723-2632. Disponível em: $<$ http://www.springerlink.com/content/6353w40m8qr58534/>.

63 DIJK, P.; BERKOWITZ, B.; BENDEL, P. Investigation of flow in water-saturated rock fractures using nuclear magnetic resonance imaging (NMRI). Water Resources Research, v. 35, n. 2, p. PP. 347-360, 1998. Disponível em: <http://www.agu.org/pubs/crossref/1999/1998WR900044.shtml>.

64 WILKE, S.; GUYON, E.; MARSILY, G. Water penetration through fractured rocks: Test of a tridimensional percolation description. Journal of the International Association for Mathematical Geology, v. 17, n. 1, p. 17-27, jan. 1985. ISSN 0020-5958. Disponível em: <http://www.springerlink.com/content/h410047262388403/>.

65 LONG, J. C. S.; BILLAUX, D. M. From field data to fracture network modeling: An example incorporating spatial structure. Water Resources Research, v. 23, n. 7, p. PP. 1201-1216, 1987. Disponível em: <http://www.agu.org/pubs/crossref/1987/WR023i007p01201.shtml>.

66 HUNT, A. G. Percolation Theory for Flow in Porous Media. EUA: Springer-Verlag, 2005.

67 BROWN, S. R.; STOCKMAN, H. W.; REEVES, S. J. Applicability of the reynolds equation for modeling fluid flow between rough surfaces. Geophysical Research Letters, v. 22, n. 18, p. PAGES 2537-2540, 1995. Disponível em: <http://www.agu.org.floyd.lib.umn.edu/journals/gl/v022/i018/95GL02666/>.

68 MOURZENKO, V.; THOVERT, J.; ADLER, P. Permeability of a single fracture; validity of the reynolds equation. Journal de Physique II, v. 5, n. 3, p. 18, 1995.

69 ORON, A. P.; BERKOWITZ, B. Flow in rock fractures: The local cubic law assumption reexamined. Water Resources Research, v. 34, n. 11, p. PP. 2811-2825, 1998. Disponível em: <http://www.agu.org/pubs/crossref/1998/98WR02285.shtml>.

70 HOLMES, D. W.; WILLIAMS, J. R.; TILKE, P. Smooth particle hydrodynamics simulations of low reynolds number flows through porous media. International Journal for Numerical and Analytical Methods in Geomechanics, v. 35, n. 4, p. 419-437, mar. 2011. ISSN 1096-9853. Disponível em: $<$ http://onlinelibrary.wiley.com/doi/10.1002/nag.898/abstract $>$.

71 CACAS, M. C.; LEDOUX, E.; MARSILY, G. de; TILLIE, B.; BARBREAU, A.; DURAND, E.; FEUGA, B.; PEAUDECERF, P. Modeling fracture flow with a stochastic discrete fracture network: calibration and validation: 1. the flow model. Water Resources Research, v. 26, n. 3, p. PP. 479-489, 1990. Disponível em: <http://www.agu.org/journals/wr/v026/i003/WR026i003p00479/>.

72 SINGHAL, B. B. S.; GUPTA, R. P. Applied Hydrogeology of Fractured Rock. EUA: Springer, 1999. 
73 HITCHMOUGH, A. M.; RILEY, M. S.; HERBERT, A. W.; TELLAM, J. H. Estimating the hydraulic properties of the fracture network in a sandstone aquifer. Journal of Contaminant Hydrology, v. 93, n. 1-4, p. 38-57, 2007. ISSN 01697722. Disponível em: <http://www.sciencedirect.com/science/article/B6V94-4MX569Y3/2/5f70b505bf8b57e246ad992b556365f0>.

74 BERKOWITZ, B.; SCHER, H. On characterization of anomalous dispersion in porous and fractured media. Water Resources Research, v. 31, n. 6, p. PP. 1461-1466, 1995. Disponível em: <http://www.agu.org/pubs/crossref/1995/95WR00483.shtml>.

75 BERKOWITZ, B.; SCHER, H. Anomalous transport in random fracture networks. Physical Review Letters, v. 79, n. 20, p. 4038, nov. 1997. Disponível em: <http://link.aps.org/doi/10.1103/PhysRevLett.79.4038>.

76 CVETKOVIC, V.; FRAMPTON, A. Transport and retention from single to multiple fractures in crystalline rock at Äspö (Sweden): 2. fracture network simulations and generic retention model. Water Resources Research, v. 46, p. 17 PP., maio 2010. Disponível em: <http://www.agu.org/pubs/crossref/2010/2009WR008030.shtml>.

77 BECKER, M. W.; SHAPIRO, A. M. Tracer transport in fractured crystalline rock: Evidence of nondiffusive breakthrough tailing. Water Resources Research, v. 36, n. 7, p. PP. 1677-1686, 2000. Disponível em: <http://www.agu.org.floyd.lib.umn.edu/journals/wr/v036/i007/2000WR900080/>.

78 POTERI, A. Retention properties of flow paths in fractured rock. Hydrogeology Journal, 2008. Disponível em: <http://dx.doi.org/10.1007/s10040-008-0414-y>.

79 WILSON, C.; WITHERSPOON, P; LONG, J.; GALBRAITH, R.; DuBois, A.; McPherson, M. Large-scale hydraulic conductivity measurements in fractured granite. International Journal of Rock Mechanics and Mining Sciences \& Geomechanics Abstracts, v. 20, n. 6, p. 269-276, 1983. ISSN 0148-9062. Disponível em: < http://www.sciencedirect.com/science/article/B6V4V-4814W8YT6/2/a9ce304512f456c932c947148c52bf62>.

80 HAKAMI, E.; LARSSON, E. Aperture measurements and flow experiments on a single natural fracture. International Journal of Rock Mechanics and Mining Science \& Geomechanics Abstracts, v. 33, n. 4, p. 395-404, jun. 1996. Disponível em: <http://www.sciencedirect.com/science/article/B6V4V4CWSPT0-4/1/47ac33ea51ba5b3902bc703c27462790>.

81 ANDO, K.; KOSTNER, A.; NEUMAN, S. P. Stochastic continuum modeling of flow and transport in a crystalline rock mass: Fanay-Augères, france, revisited. Hydrogeology Journal, v. 11, n. 5, p. 521-535, 2003. ISSN 1431-2174. Disponível em: $<$ http://www.springerlink.com/content/aec0k4qktqjw1x1p/>.

82 LANDAU, L. D.; LIFSHITZ, E. M. Fluid Mechanics. Second english. Rússia: Pergamon Press, 1987.

83 STRACK, O. D. L.; HAITJEMA, H. M. Modeling double aquifer flow using a comprehensive potential and distributed singularities: 1. solution for homogeneous permeability. Water Resources Research, v. 17, n. 5, p. PP. 1535-1549, out. 1981. Disponível em: <http://www.agu.org/pubs/crossref/1981/WR017i005p01535.shtml>.

84 STRACK, O. D. L.; HAITJEMA, H. M. Modeling double aquifer flow using a comprehensive potential and distributed singularities: 2. solution for inhomogeneous permeabilities. Water Resources Research, v. 17, n. 5, p. PP. 1551-1560, out. 1981. Disponível em: <http://www.agu.org/pubs/crossref/1981/WR017i005p01551.shtml>.

85 JANKOVIC, I. High-Order Analytic Elements in Modeling Groundwater Flow. Tese (Doutorado) University of Minnesota, 1997. 
86 CLASSICAL Potential Theory. [S.l.: s.n.].

87 CHURCHILL, R. V.; BROWN, J. W.; VERHEY, R. F. Complex Variables and Applications. EUA: McGraw-Hill, 1974.

88 REMMERT, B. R. B. R. Theory Of Complex Functions. EUA: Springer-Verlag, 1990.

89 STRACK, O. D. L. Using wirtinger calculus and holomorphic matching to obtain the discharge potential for an elliptical pond. Water Resources Research, v. 45, p. 9 PP., jan. 2009. Disponível em: $<$ http://www.agu.org/pubs/crossref/2009/2008WR007128.shtml>.

90 GUANABARA, R. C. Modelo Trasiente de Fluxo em Área de Afloramento do Sistema Aquífero Guarani. Tese (Doutorado) — Universidade de São Paulo, 2011.

91 TVEITO, A.; WINTHER, R. Introduction to Partial Differential Equations: A Computational Approach. EUA: Springer, 1998.

92 JANKOVIC, I.; BARNES, R. Three-dimensional flow through large numbers of spheroidal inhomogeneities. Journal of Hydrology, v. 226, n. 3-4, p. 224-233, dez. 1999. Disponível em: <http://www.sciencedirect.com/science/article/B6V6C-3YHFYKYD/1/61e4a1daf743cd6aa769f5c457652438>.

93 BARNES, R.; JANKOVIC, I. Two-dimensional flow through large numbers of circular inhomogeneities. Journal of Hydrology, v. 226, n. 3-4, p. 204-210, 1999. ISSN 0022-1694. Disponível em: <http://www.sciencedirect.com/science/article/B6V6C-3YHFYKYB/2/c5dcd53c0ed901aef0f50cc5f804d12e >.

94 PRESS, W. H. Numerical Recipes: the art of scientific computing. EUA: Cambridge University Press, 2007.

95 MASON, J. C.; HANDSCOMB, D. C. Chebyshev Polynomials. EUA: Chapman \& HallCRC, 2003.

96 MARIN, I. S. P. Em preparação.

97 CROUCH, S. L. Solution of plane elasticity problems by the displacement discontinuity method. i. infinite body solution. International Journal for Numerical Methods in Engineering, John Wiley \& Sons, Ltd, v. 10, n. 2, p. 301-343, 1976. ISSN 1097-0207. Disponível em: $<$ http://dx.doi.org/10.1002/nme.1620100206>.

98 JONES, E.; OLIPHANT, T.; PETERSON, P. et al. SciPy: Open source scientific tools for Python. 2001-. Disponível em: <http://www.scipy.org/>.

99 GOLUB, G. H.; LOAN, C. F. V. Matrix computations. 3rd. ed. EUA: Johns Hopkins University Press, 1996. Paperback. ISBN 0801854148. Disponível em: < http://www.worldcat.org/isbn/0801854148>.

100 ANDERSON, E.; BAI, Z.; BISCHOF, C.; BLACKFORD, S.; DEMMEL, J.; DONGARRA, J.; CROZ, J. D.; GREENBAUM, A.; HAMMARLING, S.; MCKENNEY, A.; SORENSEN, D. LAPACK Users' Guide. Third. Philadelphia, PA: Society for Industrial and Applied Mathematics, 1999. ISBN 0-89871-447-8 (paperback).

101 PYTHON. June 2011. Disponível em: <http://python.org/>.

102 BAKKER, M.; KELSON, V. A. Writing analytic element programs in python. Ground Water, v. 47, n. 6, p. 828-834, 2009. Disponível em: <http://dx.doi.org/10.1111/j.1745-6584.2009.00583.x>.

103 HUNTER, J. D. Matplotlib: A 2d graphics environment. Computing In Science \& Engineering, IEEE COMPUTER SOC, 10662 LOS VAQUEROS CIRCLE, PO BOX 3014, LOS ALAMITOS, CA 90720-1314 USA, v. 9, n. 3, p. 90-95, May-Jun 2007. 
104 DEBIAN GNU/Linux Distribution. Disponível em: <www.debian.org>.

105 ECLIPSE IDE Framework. Disponível em: <www.eclipse.org>.

106 BANDILLA, K. W.; RABIDEAU, A. J.; JANKOVIC, I. A parallel mesh-free contaminant transport model based on the analytic element and streamline methods. Advances in Water Resources, v. 32, n. 8, p. 1143-1153, 2009. ISSN 0309-1708. Disponível em: <http://www.sciencedirect.com/science/article/B6VCF-4TB18B05/2/7982ce66aa6bda7dc377ae3a07b5c61a>.

107 HERRERA, P. A.; MASSABó, M.; BECKIE, R. D. A meshless method to simulate solute transport in heterogeneous porous media. Advances in Water Resources, v. 32, n. 3, p. 413-429, mar. 2009. ISSN 0309-1708. Disponível em: <http://www.sciencedirect.com/science/article/B6VCF-4V764892/2/ced70f85726c80032121489858086ced>.

108 WONG, S.; CRAIG, J. R. Series solutions for flow in stratified aquifers with natural geometry. Advances in Water Resources, v. 33, n. 1, p. 48-54, jan. 2010. ISSN 0309-1708. Disponível em: <http://www.sciencedirect.com/science/article/B6VCF-4XJ17S71/2/bfcc82594fc98b79487e9332034ae3a0>.

109 HAITJEMA, H.; FEINSTEIN, D.; HUNT, R.; GUSYEV, M. A hybrid Finite-Difference and analytic element groundwater model. Ground Water, v. 9999, n. 9999, 2010. Disponível em: <http://dx.doi.org/10.1111/j.1745-6584.2009.00672.x>.

110 ABRAMOVITZ, M.; STEGUN, I. Handbook of Mathematical Functions. EUA: Dover Publications, 1970. 


\section{APÊNDICE A - Complementos de Hidrologia}

\section{A.1 Equação de Bernoulli e Lei de Darcy}

A equação de Bernoulli é uma forma do princípio de conservação de energia para fluidos. Dada uma massa $m$ de fluido e considerando um fluido incompressível, no campo gravitacional da Terra (considerado constante), não condutor de calor, com uma distribuição estática de pressão, de velocidade $v$, a energia mecânica da partícula de fluido com volume $\mathrm{V}$ e massa $\mathrm{m}$ é igual a

$$
p V+\frac{1}{2} m v^{2}+m g z=E
$$

Esta equação representa a energia mecânica necessária para comprimir (adiabaticamente!), elevar ou acelerar uma massa de água $m$ com relação a um referencial arbitrário onde $v=p=z=0$. $\mathrm{O}$ princípio de Bernoulli geralmente é usado para descrever o escoamento em um duto com variação de diâmetro, onde se mostra que a velocidade do fluido aumenta quando passa por uma redução no diâmetro, com consequente diminuição da pressão. É interessante notar que pressão pode ser considerada como densidade de energia:

$$
p=\frac{F}{A}=\frac{N}{m^{2}}=\frac{N \cdot m}{m^{2} \cdot m}=\frac{J}{m^{3}}
$$

ou seja, com unidades de Joule por volume. O termo de energia cinética é bem conhecido, notando apenas que agora ele é aplicado na densidade do fluido e não somente à massa. O último termo é o termo de energia potencial gravitacional do fluido.

O peso específico $\gamma$ é definido como

$$
\gamma=\rho g
$$

Dividindo a eq. de Bernoulli pelo peso $(m g=V \rho g)$, obtemos a definição de carga hidráulica, em que foi assumido que a velocidade do fluido é baixa, eliminando o termo de energia cinética:

$$
\frac{p V}{V \rho g}+\frac{1}{2} \frac{m v^{2}}{m g}+\frac{m g z}{m g}=\frac{E}{m g}=\phi \frac{p}{\gamma}+\frac{v^{2}}{2 g}+z=\phi \frac{p(x, y, z)}{\gamma}+z=\phi(x, y, z)
$$

A dissipação térmica e variações de densidade do fluido são ignoradas neste modelo, devido à baixa velocidade de escoamento e à pequena variação na energia térmica no atrito entre o fluido e o material poroso ${ }^{1}$.

\footnotetext{
${ }^{1} \mathrm{~A}$ equação de Bernoulli é válida apenas para o caso isentrópico na forma apresentada. Landau(82) mostra
} 


\section{A.1.1 Lei de Darcy}

Darcy determinou empiricamente uma expressão para a perda de energia ao fluxo específico, chamada descarga específica, $q$, a partir da área da seção porosa $A$, do caminho percorrido $L$, de uma constante de proporcionalidade $K$, chamada condutividade hidráulica, e da diferença de carga, a chamada Lei de Darcy:

$$
q=K A \frac{\phi_{1}-\phi_{2}}{L}
$$

ou

$$
q_{i}=-K \partial_{i} \phi
$$

em sua forma diferencial e em três dimensões, como pode ser visto na figura (A.1). O meio poroso serve como meio dissipativo da energia do fluido. Para estabelecer a Equação de Laplace, a equação de conservação de massa (equação de continuidade) é necessária. Juntando conservação de energia e conservação de massa a equação de Laplace é obtida $(1,52)$.

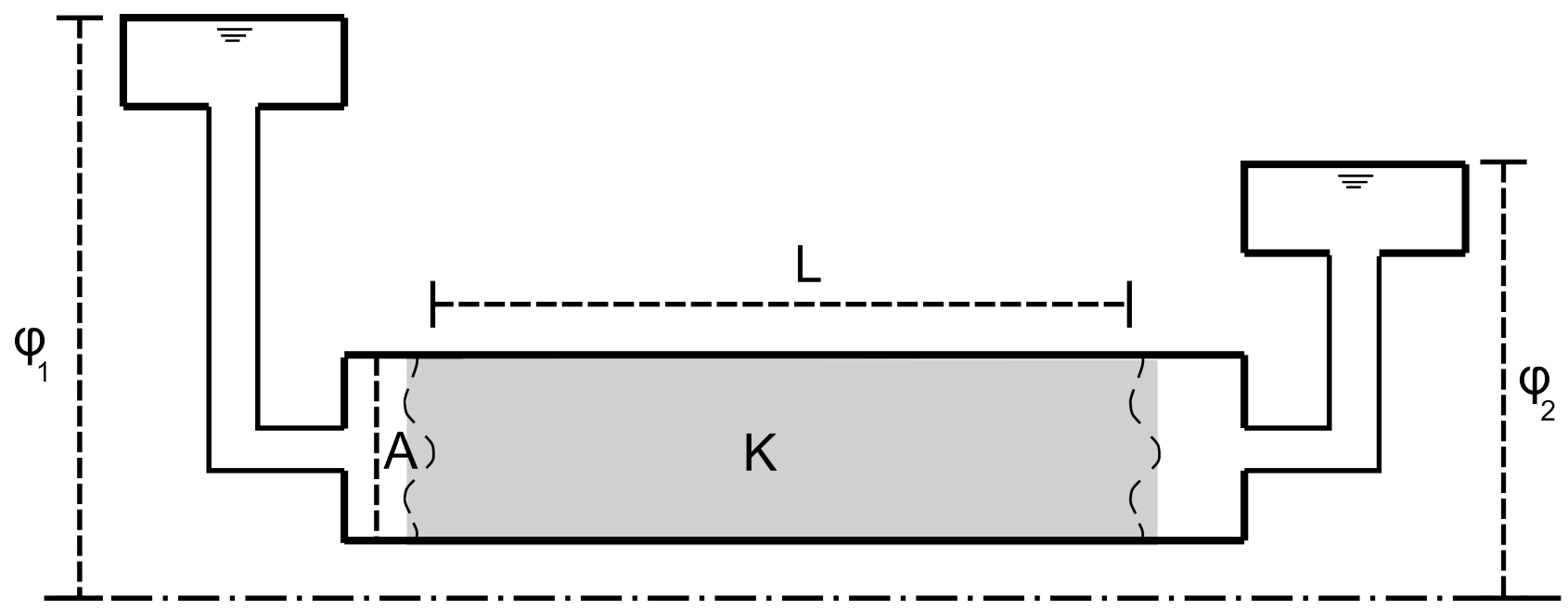

Figura A.1 - Esquema do experimento de Darcy. Adaptado de (1).

o Teorema de Bernoulli usando a entalpia para processos não-adiabáticos. 


\section{APÊNDICE B - A Aproximação de Dupuit-Forchheimer}

A aproximação de Dupuit-Forchheimer consiste em integrar o vetor descarga na direção vertical:

$$
\begin{array}{r}
Q_{i}=\int_{0}^{h} q_{i} d x_{3} \\
Q_{i}=-\int_{0}^{h} \frac{\partial K \phi}{\partial x_{i}} d x_{3}
\end{array}
$$

Usando a regra de Leibnitz,

$$
\begin{aligned}
& \frac{\partial}{\partial x} \int_{a(x)}^{b(x)} f(x, t) d t=\int_{a(x)}^{b(x)} \frac{\partial}{\partial x} f(x, t) d t+f(x, a) \frac{\partial a(x)}{\partial x}-f(x, b) \frac{\partial b(x)}{\partial x} \\
& \int_{0}^{h\left(x_{1}, x_{2}\right)} \frac{\partial}{\partial x_{i}} K \phi\left(x_{i}\right) d x_{3}=\frac{\partial}{\partial x_{i}} \int_{0}^{h\left(x_{1}, x_{2}\right)}-K \phi\left(x_{1}, x_{2}, 0\right) \frac{\partial 0}{\partial x_{i}} \\
& +K \phi\left(x_{1}, x_{2}, h\left(x_{1}, x_{2}\right)\right) \frac{\partial h\left(x_{1}, x_{2}\right)}{\partial x_{i}}
\end{aligned}
$$

ou seja, na forma vetorial,

$$
Q_{i}\left(x_{1}, x_{2}\right)=-K \vec{\nabla} h\left(x_{1}, x_{2}\right) \phi^{\prime}+K \phi\left(x_{1}, x_{2}, h\left(x_{1}, x_{2}\right)\right) \vec{\nabla} h\left(x_{1}, x_{2}\right)
$$

onde

$$
\phi^{\prime}=\frac{1}{h\left(x_{1}, x_{2}\right)} \int_{0}^{h\left(x_{1}, x_{2}\right)} \phi\left(x_{i}\right) d x_{3}
$$

Ou seja, usando o fato que

$$
\phi\left(x_{1}, x_{2}, h\left(x_{1}, x_{2}\right)\right)=h\left(x_{1}, x_{2}\right)
$$

na superfície livre $(p=0)$,

$$
Q_{i}(x, y)=-K \vec{\nabla} h \phi^{\prime}+K h \vec{\nabla} h=-\vec{\nabla}\left(K h \phi^{\prime}-K \frac{h^{2}}{2}\right)
$$

Considerando um aquífero não confinado e assumindo agora a aproximação de Dupuit-Forchheimer, 
$\phi^{\prime}=h\left(x_{1}, x_{2}\right)$, o que equivale a equipotenciais verticais, e portanto

$$
\Phi=K H \phi^{\prime}-K \frac{h^{2}}{2}=\frac{K h^{2}}{2}
$$

A média $\phi^{\prime}$ não é igual à $h$, sendo somente igual quando se assume que as equipotenciais são verticais, ou seja, a média da carga na direção z é igual ao valor da carga $h$ no ponto onde a pressão é igual a pressão atmosférica. 


\section{APÊNDICE C - Matriz de Interação para uma Line Doublet}


$\Phi_{\pi}^{\infty} \Phi^{-1} \Phi^{2} \Phi^{2} \Phi^{2}$

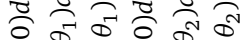

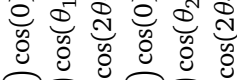

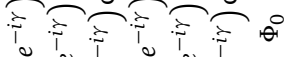

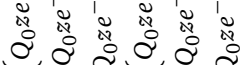

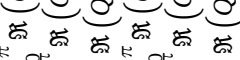

$\underbrace{0 \underbrace{\circ}}$

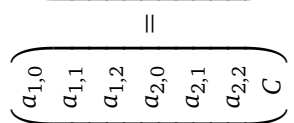

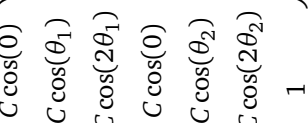

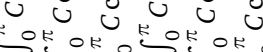

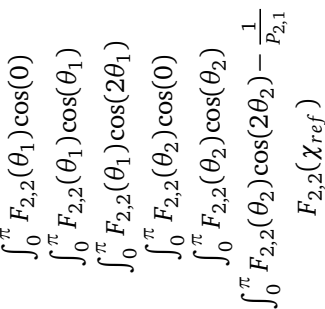
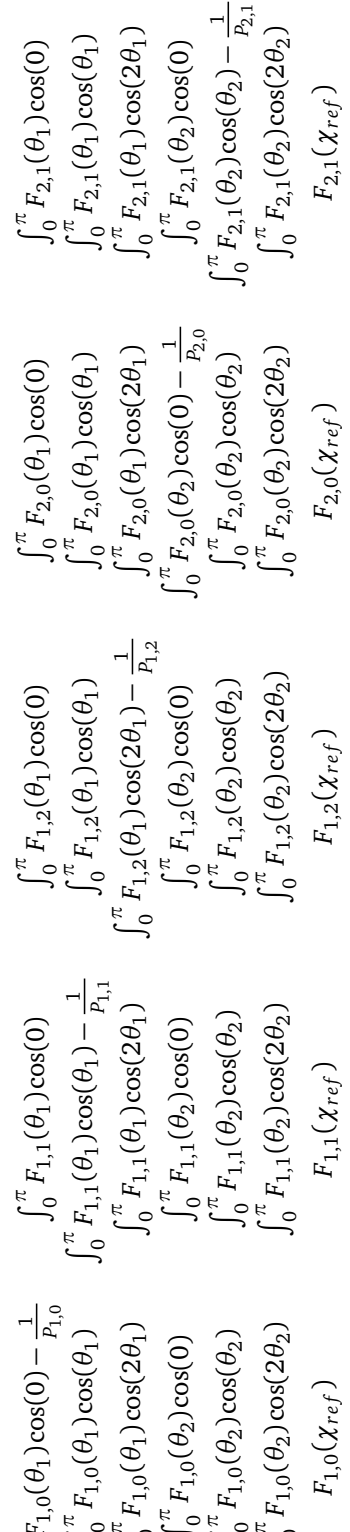


\section{APÊNDICE D - Relações de Ortogonalidade}

As relações de ortogonalidade usadas neste trabalho estão descritas abaixo. Estas expressões podem ser encontradas em (110).

\section{D.1 Relações de Ortogonalidade Contínuas}

$$
\begin{aligned}
& \int_{0}^{\pi} \sin (m \theta) \sin (n \theta) d \theta=\frac{\pi}{2} \delta_{m n} \\
& \int_{0}^{\pi} \cos (m \theta) \cos (n \theta) d \theta=\frac{\pi}{2} \delta_{m n} \\
& \int_{0}^{2 \pi} \sin (m \theta) \sin (n \theta) d \theta=\pi \delta_{m n} \\
& \int_{0}^{2 \pi} \cos (m \theta) \cos (n \theta) d \theta=\pi \delta_{m n} \\
& \int_{0}^{2 \pi} e^{i m \theta} e^{i n \theta} d \theta=\pi \delta_{m n}
\end{aligned}
$$




\section{D.2 Relações de Ortogonalidade Discretas}

$$
\begin{aligned}
& \sum_{k=0}^{K} \sum_{n=0}^{N} \sum_{m=0}^{M} \sin \left(m \theta_{k}\right) \sin \left(n \theta_{k}\right)=K \delta_{n m} \\
& \sum_{k=0}^{K} \sum_{n=0}^{N} \sum_{m=0}^{M} \cos \left(m \theta_{k}\right) \cos \left(n \theta_{k}\right)=K \delta_{n m}
\end{aligned}
$$

para

$$
\theta_{k}=\frac{2 \pi k}{K}
$$




\section{APÊNDICE E - Desenvolvimentos Adicionais}

\section{E.1 Simulador do Método de Elementos Analíticos - SME- Agol e o Visu}

O Método de Elementos Analíticos foi implementado usando o método da colocação, chamado de SMEAgol, Simulador de Métodos de Elementos Analíticos. No SMEAgol foram implementadas o elemento para poço, line sink e inhomogeneidades poligonais usando line doublets. Sua implementação foi feita em $\mathrm{C}++$, aplicando todos os conceitos de programação orientada a objetos, como polimorfismo e herança. O solver usado para resolver o sistema linear foi o LAPACK (100). Apesar de resultados satisfatórios para os elementos implementados, seu desenvolvimento foi descontinuado, devido às complexidades envolvidas em sua estrutura orientada a objetos, e a consequente dificuldade em adaptar esta estrutura para o novo formalismo de solução para os elementos analíticos apresentado nesta tese.

As figuras (E.1), (E.2) e (E.3) mostram gráficos gerados pelo Visu, um visualizador de isolinhas e superfícies de carga, implementado também durante este trabalho usando os padrões OpenGL e Qt em C++. O Visu permite a rotação das superfícies geradas, permitindo a visualização em 2,5D da superfície de carga, na tentativa de permitir um entendimento mais fácil. O Visu também implementa um traçador de linhas de corrente, dado um arquivo de entrada com valores de velocidade para pontos na superfície. 


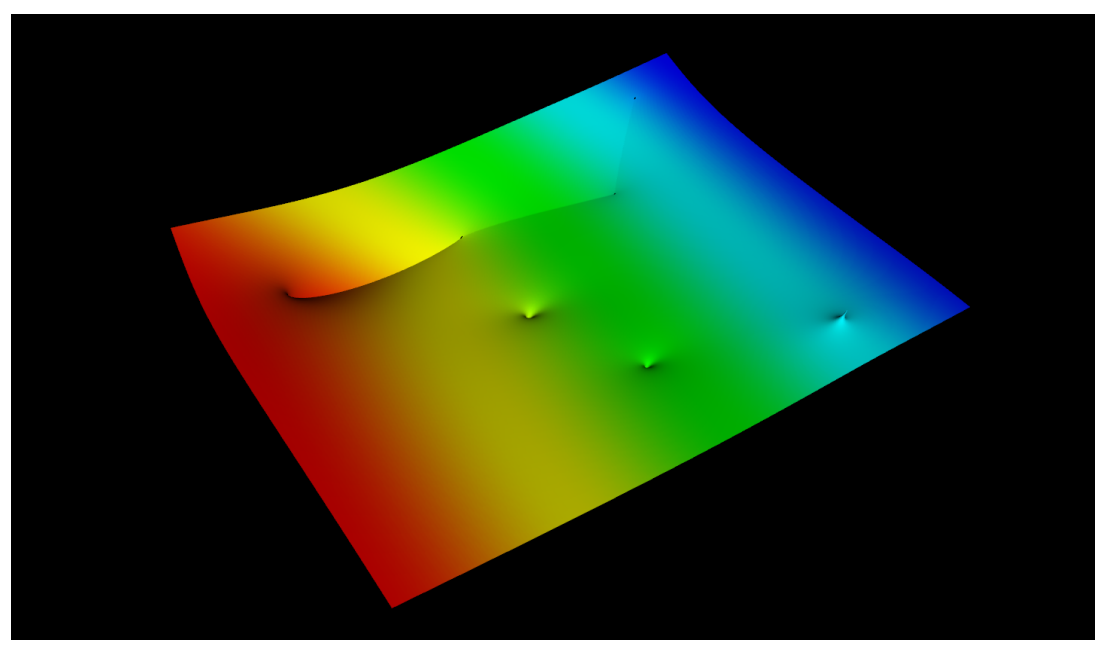

Figura E.1 - Gráfico gerado pelo Visu a partir da simulação SMEAgol. Isosuperfície de carga com três poços, line sinks e um campo de escoamento uniforme.

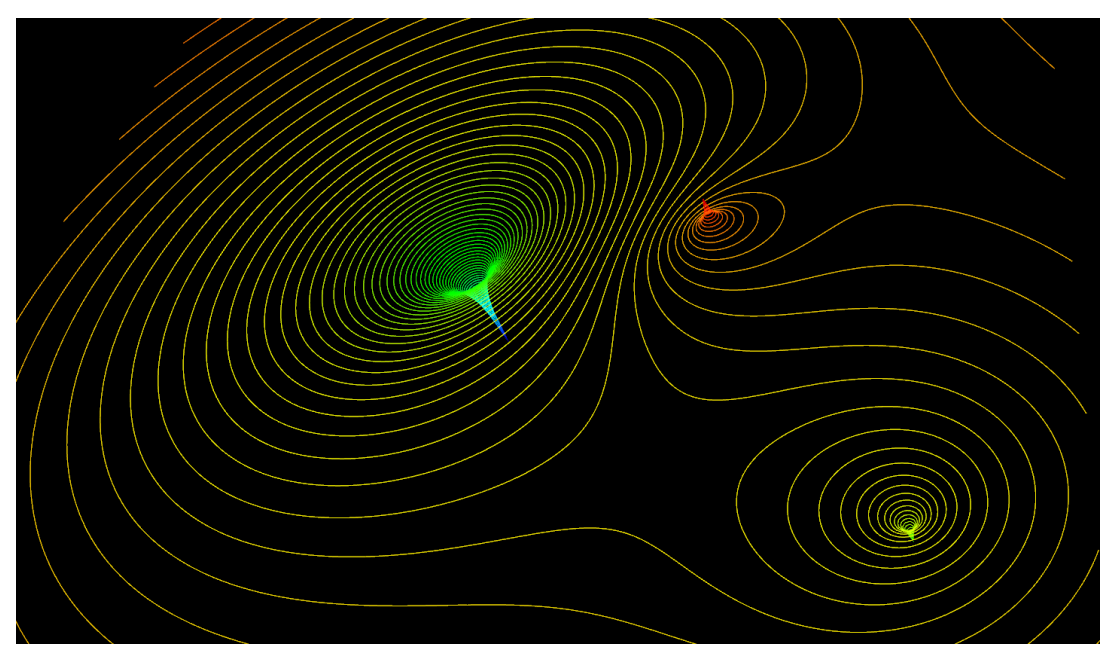

Figura E.2 - Gráfico gerado no Visu a partir dos resultados do SMEAgol. Isolinhas de carga para um sistema com três poços, dois extratores e um injetor.

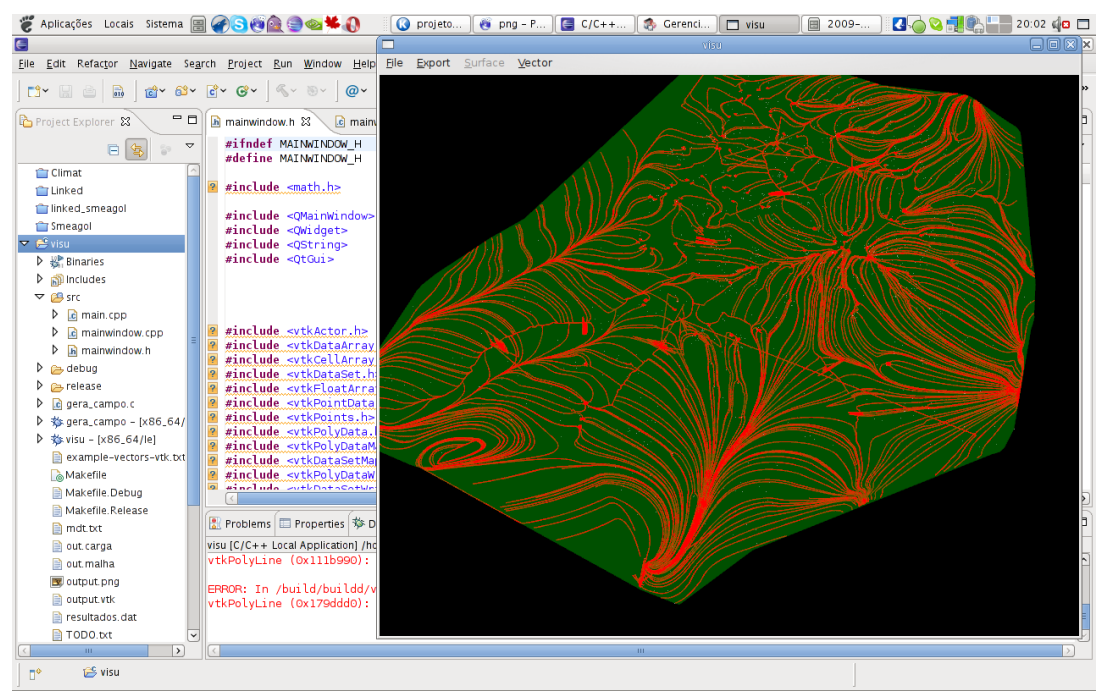

Figura E.3 - Gráfico gerado a partir do Visu e de um arquivo de dados com velocidades. Linhas de Corrente. 